\title{
Studies of Seismic Velocities in Subduction Zones from Continuous Ocean Bottom Seismometer Data
}

\author{
by \\ Weiwei Wang
}

\author{
A thesis \\ submitted to Victoria University of Wellington \\ in fulfillment of the \\ requirements for the degree of \\ Doctor of Philosophy \\ in Geophysics
}

School of Geography, Environment and Earth Science

Victoria University of Wellington

Wellington, New Zealand 



\section{Abstract}

This thesis uses continuous ambient noise data recorded by Ocean Bottom Seismometers (OBSs) to study seismic velocities in the upper crust of the overriding plate. The first and second projects (Chapters 3 and 4) focus on temporal seismic velocity variations in the northern Hikurangi subduction zone offshore the North Island, New Zealand, while the third project (Chapter 5) focuses on shear wave velocities in the southwestern Okinawa Trough offshore northeastern Taiwan.

In the first project (Chapters 3), we investigate a region of frequent slow slip events (SSEs) offshore Gisborne, North Island, New Zealand. From September to October 2014, an SSE occurred with a slip over $250 \mathrm{~mm}$ and was recorded successfully by the Hikurangi Ocean Bottom Investigation of Tremor and Slow Slip deployment II (HOBITSS II). We apply coda wave interferometry on the ambient noise data acquired by nine OBSs deployed by the HOBITSS II to study the seismic velocity variations related to the SSE. The average velocity variations display a decrease on the order of $0.05 \%$ during the SSE, followed by an increase of similar magnitude afterwards. Two hypotheses are proposed to explain our observation. The first hypothesis, which has been suggested by previous studies, considers that the velocity decrease during the SSE is caused by more fluids migrating into the upper plate as the SSE breaks a low-permeability seal on the plate boundary. After the SSE, the fluids in the upper plate diffuse gradually and the velocity increases; The second hypothesis is that before the SSE, elastic strain accumulates causing contraction and reduction of porosity and therefore increase of velocity (the velocity increase between SSEs). During the SSE, the velocity decrease is caused by increased porosity as the SSE relieves the accumulated elastic strain on the plate interface, which results in dilation. After the SSE, stress and strain accumulate again, causing a porosity decrease and a velocity increase back to the original value. This study demonstrates that the velocity variations related to SSEs are observable and 
provides evidence for slow slip mechanism hypotheses.

The second project (Chapter 4) focuses on the temporal seismic velocity variations associated with an SSE in 2019 offshore Gisborne, North Island, New Zealand. This is a later SSE in the same area as the first project (Chapters 3). Based on the success of the HOBITSS II, more ocean bottom instruments were deployed in the northern Hikurangi subduction zone from 2018 to 2019 (HOBITSS V). An SSE lasting approximately one month from the end of March to the beginning of May 2019 occurred during the deployment and was recorded by the network. The main slip was south of the deployment and the slip beneath the deployment was up to $150 \mathrm{~mm}$. This study applies coda wave interferometry on the ambient noise data acquired by five OBSs and computes seismic velocity variations to investigate their relation to the SSE. A velocity decrease on the order of $0.015 \%$ during the SSE and an increase back to the original velocity value are observed at 1-2.5 $\mathrm{s}$. This supports the two hypotheses proposed in Chapters 3: fluid migration and/or stain changes through the SSE cycle. In addition, velocity variations computed from individual stations show velocity increases before the SSE, which are destructive interfered in their average. Such a situation could occur if the SSE migrated across the network. If the velocity increases before the SSE from individual stations are real, they can be only explained by the hypothesis of crustal strain changes (the second hypothesis in project 1). However, fluid migration (the first hypothesis in project 1) may still happen concomitantly.

The third project focuses on the tectonics in southwestern Okinawa Trough offshore northeastern Taiwan. The southwestern Okinawa Trough is an active backarc basin, extending and rifting within the continental lithosphere. The tectonic development of the back-arc basin is still not well-understood. This study uses continuous ambient noise data recorded by 34 OBSs deployed by Academia Sinica at various periods from 2010 to 2018. Cross-correlations on vertical seismic components and pressure gauges are computed to construct Rayleigh/Scholte waves to study the shear wave velocity structure in the southwestern Okinawa Trough. Phase velocities are measured from the Rayleigh/Scholte waves. Shear velocities are inverted from the phase velocities. Results show the velocity in the south of the back-arc rifting axis near the axis is slower than the velocity in the north of the rifting axis, suggesting the velocity structure in the southwestern Okinawa Trough is asymmetric along the rifting axis. Previous studies have shown high heat flows 
(about $110 \mathrm{~mW} / \mathrm{m}^{2}$ on average) in the south of the rifting axis. The low velocity in the south could be caused by the high heat flow that may be related to asymmetric back-arc extension and/or rifting. This study presents the shear wave velocity structure in the southwest Okinawa Trough is asymmetric along the rifting axis, which implies the back-arc extending/rifting is asymmetric in the study region. This study also suggests effective techniques for OBS noise corrections and unwrapping the cycle skipping of phase velocity measurements.

In summary, this thesis represents three projects focusing on seismic velocities in two subduction zones using ambient noise data collected by OBSs. The first and second projects study the temporal velocity variations and the relation to SSEs. Both studies observe velocity decreases during the SSEs and increases after the SSEs, supporting two hypotheses of fluid migration and/or stain changes through the SSE cycle. The third project finds the shear velocity structure in the southwestern Okinawa Trough is asymmetric along the rifting center, which may imply the back-arc extension is asymmetric. 


\section{Acknowledgments}

First, I would like to acknowledge my supervisors Martha Savage, Tim Stern, and Bill Fry, who provided guidance and suggestions to my Ph.D.. They provided me information and available resources that were helpful to my research and career. My primary supervisor Martha Savage has been very supportive and tolerant, especially during my first year. As I concentrated more on methodology research before I started my Ph.D., she guided me to the tectonic research required for my thesis. She helped me to get used to working in New Zealand. She tolerated the mistakes that I made because of cultural differences. I have had a lot of conversations with her on different topics, from which she also provided suggestions on my career and life.

My collaborators and colleagues have helped me through my Ph.D. journey. I acknowledge my collaborators Martha Savage, Shu-Huei Hung, Alec Yates, Yinhe Luo, Hubert Zal, Ban-Yuan Kuo, Xiaozhou Yang, Spahr Webb, Carolyn Boulton, Emily Warren-Smith, Megan Kortink, Tim Stern, Bill Fry, Kimihiro Mochizuki, Laura Wallace, Hao Rao, Pei-Ying Patty Lin, and Hsin-Ying Yang. I acknowledge my colleagues at Victoria University of Wellington, GNS Science, and The University of Edinburgh, Hubert Zal, Kenny Graham, Katie Woods, Pasan Herath, Katrina Jacobs, Emily Warren-Smith, Laura Wallace, Bryant Chow, Andrea Perez, Laura-May Baratin, Francesco Civilini, Marjolaine Verret, Hannah Chorley, Chet Hopp, Calum Chamberlain, Hamish Hirschberg, El Mestel, Jesse Kearse, Finn Illsley-Kemp, Andrew Curtis, and Xin Zhang. I also acknowledge VUW career coaches Louise Taylor, Alice Hodder, Millie Douglas, and Maaike Christie-Beekman.

During my time in Wellington, my family has constantly supported me, especially this year after January when we started frequent video calls. My parents have been patient and kind to me. My brother Xiaofei, as an HR, has provided me advice on 
job hunting even though he is very busy with his work. My niece Zixuan Yuan is also nice to me. My friends in Wellington, Shirley Wang, Emily Fe, Chen Ding, Sephy He, Aasha Pancha, Yang Bai, May Jin, Pei-Ying Pan, and Bill Wang, have been strong support. Shirley has been accommodating with everything. She made my life much easier in Wellington.

Last, I would like to acknowledge Yinhe Luo and his students Xiaozhou Yang, Yao Huang, Jinyun Xie, Kaifeng Zhao, Jing Lin. Thank you for always being with me. I hope I will be able to stay in Geophysics in the future. 


\section{Contents}

Abstract

Acknowledgments vii

$\begin{array}{ll}\text { Contents } & \text { ix }\end{array}$

List of Figures $\ldots \ldots \ldots \ldots \ldots \ldots \ldots \ldots$ xiii

List of Tables . . . . . . . . . . . . . . . . . . xix

1 Introduction 1

1.1 Motivation . . . . . . . . . . . . . . . . . 1

1.2 Slow slip event ..................... 2

1.3 Velocity variations $\ldots \ldots \ldots \ldots \ldots$

1.4 Shear velocity structure . . . . . . . . . . . . . 5

1.5 Tectonic setting in the Hikurangi margin, New Zealand . . . . . . . 5

1.6 Tectonic setting offshore Taiwan . . . . . . . . . . . . . . 9

1.7 Comparison of the Hikurangi and Taiwan margins . . . . . . . . . . 12

1.8 Thesis structure $\ldots \ldots \ldots \ldots \ldots$

2 Data and methodology 19

2.1 Data acquisition . . . . . . . . . . . . . . . . 19

2.1 .1 HOBITSS ......................... 19 
2.1.2 Southwestern Okinawa Trough deployment . . . . . . . . 20

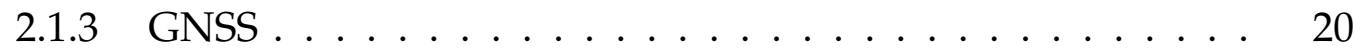

2.2 Ambient noise . . . . . . . . . . . . . . . . 23

2.3 MSNoise . . . . . . . . . . . . . . . . . . 27

2.4 Cross-correlation functions $\ldots \ldots \ldots$. . . . . . . . . 27

2.5 Velocity variations . . . . . . . . . . . . . . . . . . . . 29

2.6 Phase velocity measurement and shear velocity inversion . . . . . . 31

2.6.1 Phase velocity measurement . . . . . . . . . . 32

2.6.2 Bayesian inversion and Markov Chain Monte Carlo . . . . . 33

2.7 1D sensitivity kernel $\ldots \ldots \ldots \ldots$. . . . . . . . . . . . . 34

2.8 Data and method comparison of the two study regions . . . . . . 35

3 Temporal velocity variations in the northern Hikurangi margin and the relation to slow slip 37

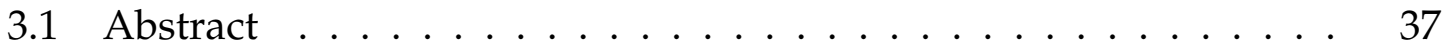

3.2 Introduction $\ldots \ldots \ldots \ldots \ldots \ldots$

3.3 Data . . . . . . . . . . . . . . . . . . . . . 41

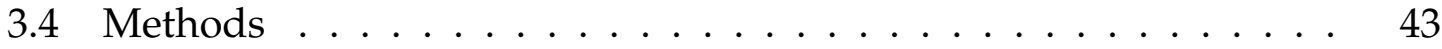

3.5 Results .......................... 46

3.6 Discussion . . . . . . . . . . . . . . . . 47

3.6 .1 Relation to SSEs . . . . . . . . . . . . . . . . 47

3.6.2 Fluid migration related to fault-valve behavior . . . . . . . 52

3.6.3 Assessing our observations in terms of porosity changes due to crustal strain . . . . . . . . . . . . . 56

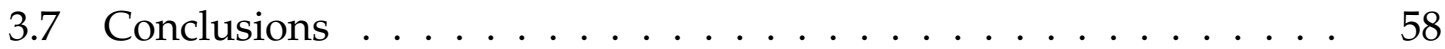

3.8 Acknowledgments . . . . . . . . . . . . . . . . . . 59 
4 Temporal velocity variations in 2018-2019 in the northern Hikurangi margin and the relation to slow slip

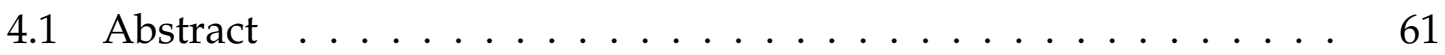

4.2 Introduction . . . . . . . . . . . . . . . . . 62

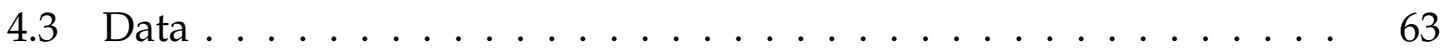

4.4 Methods ............................ 64

4.4 .1 Parameterization . . . . . . . . . . . . . . 64

4.4 Data processing ................. 70

4.5 Results ........................... 76

4.6 Discussion . . . . . . . . . . . . . . 76

4.6.1 Depths of velocity variations . . . . . . . . . 76

4.6.2 Velocity increases before the SSE . . . . . . . . . . . 79

4.6 .3 Porosity changes . . . . . . . . . . . . . . . 80

4.7 Conclusions . . . . . . . . . . . . . . . . 82

5 Studies of shear wave velocity structure in the southwestern Okinawa $\begin{array}{lr}\text { Trough from continuous OBS data } & 85\end{array}$

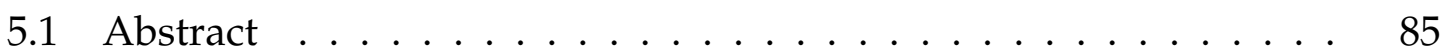

5.2 Study region and tectonic background . . . . . . . . . . . 86

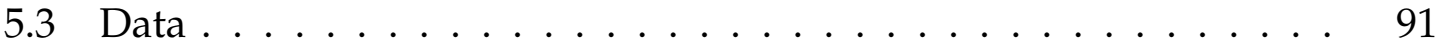

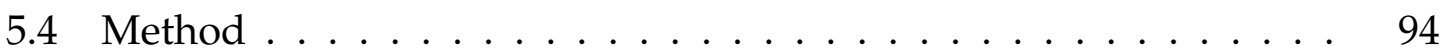

5.4.1 Ambient noise cross-correlations . . . . . . . . . . . . . 94

5.4.2 Correction for compliance and tilt noise . . . . . . . . . . 100

5.5 Results . . . . . . . . . . . . . . . . . . . 108

5.5.1 Phase velocity dispersion curves and 1D inversion . . . . . 108

5.6 Discussion . . . . . . . . . . . . . . . . . . . 121 
5.6.1 Higher mode Rayleigh/Scholte waves . . . . . . . . . . . . . 121

5.6 .2 Sensitivity kernels . . . . . . . . . . . . . . . . . . 124

5.6 .3 Volcano ....................... 125

5.6 .4 High heat flow . . . . . . . . . . . . . . . . 125

5.7 Conclusions . . . . . . . . . . . . . . . . 126

6 Conclusions $\quad 129$

$\begin{array}{ll}\text { Appendices } & 135\end{array}$

A Appendix A: Chapter 3 . . . . . . . . . . . . . . 135

B Appendix B: Chapter $4 \ldots \ldots \ldots 151$

C Appendix C: Chapter 5 . . . . . . . . . . . . . . 157

D Appendix D: Glossary . . . . . . . . . . . . . . . . . . 169

$\begin{array}{ll}\text { References } & 171\end{array}$ 


\section{List of Figures}

1.1 The seismogenic zones of earthquakes and SSEs . . . . . . . . 4

1.2 Tectonic settings in the Hikurangi margin and seamount distribution 7

1.3 SSE recordings in the northern Hikurangi margin $\ldots \ldots$. . . . 8

1.4 Taiwan Tectonic settings . . . . . . . . . . . . . . . . 10

1.5 Shear wave velocity models in the southwestern Okinawa Trough . 11

1.6 Wedge-shaped opening of the Okinawa Trough and the Central Volcanic Region . . . . . . . . . . . . . . . . . . . . 14

2.1 Stations used in Chapter 3 and Chapter $4 \ldots \ldots 21$

2.2 Stations used in Chapter $5 \ldots \ldots \ldots 22$

2.32 2-D illustration of ambient noise interferometry . . . . . . . . 25

2.4 Unevenly distributed noise sources . . . . . . . . . . . . . . . 26

2.5 Noise power spectral densities . . . . . . . . . . . . . 26

$2.6 \mathrm{dt} / \mathrm{t}$ measurement . . . . . . . . . . . . . . . . . 31

2.7 Illustration of group and phase velocities $\ldots \ldots . \ldots 32$

3.1 Locations of ocean bottom seismometers 2014-2015 _ . . . . . . . 42

3.2 20-day current stacks over time and corresponding reference stack $\quad 45$

3.3 Sensitivity kernels of fundamental mode Rayleigh . . . . . . . . . . 46

3.4 Sensitivity kernels of the first overtone Rayleigh wave . . . . . . . . 47 
3.5 Single station time-dependent velocity variations and their average 48

3.6 Temporal velocity variations compared to other studies . . . . . . . 51

3.7 Interpretation schematic models $\ldots \ldots \ldots 55$

4.1 Locations of ocean bottom seismometers 2018-2019 _ . . . . . . . . 64

4.2 SNR of different filters . . . . . . . . . . . . . . . . . 66

4.3 Correlation coefficients of $1-2.5 \mathrm{~s}$ band . . . . . . . . . . . . . 66

4.4 Correlation coefficients of $2.5-20 \mathrm{~s}$ band . . . . . . . . . . . . . 67

4.5 Determination of coda wave window and threshold of coherence for $1-2.5$ s filter . . . . . . . . . . . . . . . . . 67

4.6 Similar to Figure 4.5 but for filter $2.5-20 \mathrm{~s} \ldots \ldots \ldots$

4.7 20-day current stacks of 1-2.5 s and corresponding reference stack . 72

4.8 20-day current stacks of 2.5-20 s and corresponding reference stack 73

4.9 Reference stacks of $1-2.5 \mathrm{~s}$ on the three components . . . . . . . . 74

4.10 Reference stacks of $2.5-20 \mathrm{~s}$ on the three components $\ldots . . . . .75$

4.112019 SSE timing from GNSS data $\ldots \ldots \ldots 77$

4.12 Velocity variations at $1-2.5 \mathrm{~s} \ldots \ldots \ldots 77$

4.13 Velocity variations at $2.5-14 \mathrm{~s} \ldots \ldots \ldots 78$

4.14 Velocity variations in 2014-2015 computed using only EOBSs . . . . 78

4.15 Interpretation schematic models $\ldots \ldots$. . . . . . . . . . . . 81

4.16 Locations of bottom pressure recorders 2018-2019 _ . . . . . . . . . 82

5.1 Tectonics northeastern offshore Taiwan _ . . . . . . . . . . . . . 89

5.2 Hot vents in southwestern Okinawa Trough . . . . . . . . . . . . . . 90

5.3 Deployment of ocean bottom seismometers in the southwestern Okinawa Trough . . . . . . . . . . . . . . . . . . 92

5.4 Instrument responses . . . . . . . . . . . . . . . . . . . . 93 
5.5 Ambient noise power spectral density . . . . . . . . . . . . . 94

5.6 Comparison of Root-Mean-Square and 1-bit normalization . . . . . 96

5.7 Comparison of one, three, and five times Root-Mean-Square . . . . 97

5.8 Comparison of the phase of linear stack and phase-weighted stack 98

5.9 Comparison of different correlation thresholds . . . . . . . . . . 98

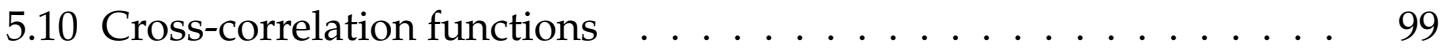

5.11 Cartoon illustration of two major noise sources on OBS data . . . . 101

5.12 Workflow of tilt and compliance noise correction . . . . . . . . . 103

5.13 Power spectral density and pressure-vertical coherence . . . . . . . 104

5.14 Power spectral density and waveform comparison of cross-correlations computed by uncorrected data and corrected data . . . . . . . . . 106

5.15 Waveform comparison of cross-correlations computed by uncorrected data and all-frequency-range corrected data . . . . . . . . . . 107

5.16 Comparison of cross-correlation functions computed by raw data and compliance-corrected data . . . . . . . . . . . 107

5.17 Unwrapping the phase . . . . . . . . . . . . . . . . . 109

5.18 Phase velocity dispersion curves measured from all the cross-correlation functions . . . . . . . . . . . . . . . . . . 110

5.19 Averaged phase velocity dispersion curves of fundamental mode Scholte wave and the possible 1st overtone Scholte wave . . . . . . 110

5.20 Station-pair path coverage . . . . . . . . . . . . . . . . . 112

5.21 Phase velocity dispersion curves in the three sub-regions . . . . . 113

5.22 Vs inversion in the center region using the fundamental mode . . . 116

5.23 Vs inversion in the northwest edge region using the fundamental mode ........................... 117

5.24 Vs inversion in the south region using the fundamental mode . . . 117 
5.25 Vs inversion in the center region using two modes . . . . . . . . . 118

5.26 Mean Vs models of the three sub-regions _ . . . . . . . . . . . . 120

5.27 Comparison of forward-modeled dispersion curves and observed dispersion curves in the center region . . . . . . . . . . . . 122

5.28 Comparison of forward-modeled dispersion curves and observed dispersion curves in the northwest edge region . . . . . . . . . . . 122

5.29 Comparison of forward-modeled dispersion curves and observed dispersion curves in the south region . . . . . . . . . . . . . . . . 123

5.30 Sensitivity kernels based on the inverted shear velocity models in the three sub-regions . . . . . . . . . . . . . . . . . 124

5.31 Spatial distribution of velocity findings and tectonic interpretation

A.1 Instrument responses of vertical seismometers and pressure gauges of LOBSs and EOBSs . . . . . . . . . . . . . . . . . . 135

A.2 Locations of SSE1, SSE3, and SSE4 . . . . . . . . . . . . . . 137

A.3 Velocity variation measurement . . . . . . . . . . . . . . . . . . 138

A.4 SNR of different filters . . . . . . . . . . . . . . . . . . . 142

A.5 Lag-time dependent SNR of different segmentation lengths and overlaps .............................. 143

A.6 Relationship between stack sizes and correlation coefficients . . . 143

A.7 Available single station cross-component correlations . . . . . . . . 144

A.8 Reference stacks on the three components . . . . . . . . . . . . . . . 144

A.9 Determination of coda wave window and threshold of coherence . 145

A.10 Comparison of velocity variations using different reference stacks . 145

A.11 Comparison of velocity variations computed at $1-2.5 \mathrm{~s}$ and $2.5-14 \mathrm{~s} \quad 145$

A.12 Comparison of velocity variations computed at 2.5-20 s and 2.5-14 s 146

A.13 Comparison of velocity variations computed at 2.5-6 s and 6-14 s . 146 
A.14 Comparison of velocity variations computed by LOBSs and EOBSs 146

A.15 Velocity variations compared with pressure data . . . . . . . . . 147

A.16 Comparison to the velocity variations computed including cross station correlations . . . . . . . . . . . . . . . . . . . . . 148

A.17 Comparison to the velocity variations computed from EOBS . . . . 149

A.18 Comparison to the velocity variations computed from LOBS . . . . 149

A.19 Comparison to the velocity variations computed using cross-station correlations only . . . . . . . . . . . . . . . . . . . . . . 149

B.1 Earthquakes during the HOBITSS V deployment . . . . . . . . . . . 152

B.2 Single-station-average velocity variations at $1-2.5 \mathrm{~s} \quad \ldots \ldots \ldots$

B.3 Average velocity variations computed including cross station correlations, single station cross-component correlations, and auto-correlations, filtered at $1-2.5 \mathrm{~s} \ldots \ldots \ldots$. . . . . . . . . . . . . . 153

B.4 Examples of velocity variations computed from individual stationpairs, filter at $1-2.5 \mathrm{~s} \ldots \ldots$. . . . . . . . . . . . . 154

B.5 Average velocity variations computed including cross station correlations, single station cross-component correlations, and auto-correlations, filter at $2.5-14 \mathrm{~s} \ldots \ldots \ldots$. . . . . . . . . . . . . . . . 155

B.6 Examples of velocity variations computed from individual stationpairs, filtered at $2.5-20 \mathrm{~s} \ldots \ldots \ldots 156$

C.1 Vs inversion in the center region using two modes, assuming the earlier arrivals are the 2 nd overtone . . . . . . . . . . . . . . . . 159

C.2 Vs inversion in the center region using two modes, assuming the earlier arrivals are the 3rd overtone . . . . . . . . . . . . . . . 160

C.3 Vs inversion in the center region using only the earlier arrivals, assuming the earlier arrivals are the 1 st overtone . . . . . . . . . . . 160 
C.4 Comparison of Vs models inverted with different assumptions in the center region . . . . . . . . . . . . . . . . . 161

C.5 Vs inversion in the northwest edge region using two modes, assuming the earlier arrivals are the 1 st overtone . . . . . . . . . . . . 162

C.6 Vs inversion in the northwest edge region using two modes, assuming the earlier arrivals are the 2 nd overtone . . . . . . . . . . . . 162

C.7 Vs inversion in the northwest edge region using two modes, assuming the earlier arrivals are the 3 rd overtone $\ldots \ldots$. . . . . . . 163

C.8 Vs inversion in the northwest edge region using only the earlier arrivals, assuming the earlier arrivals are the 1st overtone . . . . . . 163

C.9 Comparison of Vs models inverted with different assumptions in the northwest edge region . . . . . . . . . . . . . . . . 164

C.10 Vs inversion in the south region using two modes, assuming the earlier arrivals are the 1 st overtone $\ldots \ldots$. . . . . . . . . 165

C.11 Vs inversion in the south region using two modes, assuming the earlier arrivals are the 2 nd overtone $\ldots \ldots$. . . . . . . . . . . 165

C.12 Vs inversion in the south region using two modes, assuming the earlier arrivals are the 3 rd overtone . . . . . . . . . . . . . . . 166

C.13 Vs inversion in the south region using only the earlier arrivals, assuming the earlier arrivals are the 1 st overtone . . . . . . . . . 166

C.14 Comparison of Vs models inverted with different assumptions in the south region . . . . . . . . . . . . . . 167 


\section{List of Tables}

1.1 Comparison of SSEs in different subduction zones . . . . . . . . . 3

1.2 Comparison of the Hikurangi and Taiwan margins . . . . . . . . . . 12

1.3 Comparison of Southwestern Okinawa Trough and Central Volcanic Region $(\mathrm{NZ}) \ldots \ldots \ldots \ldots \ldots$

2.1 Comparison of data and methods used in the two study regions . . 35

3.1 Comparison of Rivet et al. (2011) and this study . . . . . . . . . 58

4.1 Final parameters used for the HOBITSS V data . . . . . . . . . 69

5.1 Deployment and instrumentation information . . . . . . . . . 93

5.2 The prior setting for $\mathrm{Vs}$ inversion . . . . . . . . . . . . . 115

5.3 Mean Vs and corresponding standard deviations . . . . . . . . . . 119

A.1 Locations of the ocean bottom seismometers deployed by the HOBITSS II . . . . . . . . . . . . . . . . . . . . . . . . 136

A.2 Velocity and density models used to compute sensitivity kernels . . 136

A.3 Final parameters used for the HOBITSS II data . . . . . . . . . . . 141

B.1 Locations of the ocean bottom seismometers deployed by the HOBITSS V . . . . . . . . . . . . . . . . . . . . . . . . . 151

C.1 Locations of the ocean bottom seismometers used in Chapter 5 . . . 158 
D.1 Acronyms used in thesis . . . . . . . . . . . . . . . 169 


\section{Chapter 1}

\section{Introduction}

\subsection{Motivation}

About $70 \%$ of the earth's total surface area is covered by the ocean with no permanent seismograph observations. However, most plate boundaries with frequent seismic events are under the sea, and a large portion of the oceanic lithosphere is far from the plate boundary but seismically inactive. Therefore, deploying dense arrays of Ocean Bottom Seismometers (OBSs) on the seafloor within a region of interest plays an increasingly important role. In the past 20 years, the seismic interferometry technique has been developed rapidly and is now widely used. Stations can be considered as virtual sources to reproduce the earth's response between each virtual source and the rest of the stations by cross-correlation of passive noise fields recorded during the same time period. Due to the development of OBS instrumentation and the seismic interferometry technique, shallow seismic structures below the seafloor in many oceanic regions have started to be investigated in detail (e.g., Duennebier and Sutton, 1995; Harmon et al., 2007; Shinohara et al., 2008; Bell et al., 2015; Yang et al., 2020). Subduction under the sea can be observed with higher resolution through OBS deployments.

This these focuses on seismic velocities in the northern Hikurangi margin and southwestern Okinawa Trough. The studies can improve our understanding to slip and subduction behaviours, which will contribute to hazard assessment and tectonic study. In particular, this thesis addresses the following questions: 
1. What is the best practice for processing ocean bottom seismometer ambient noise data?

2. How does the occurrence of slow slip events influence seismic velocities? Are velocity variations associated with the occurrence of slow slip events in New Zealand detectable?

3. What are the physical processes by which slow slip events cause velocity variations?

4. What is the seismic velocity structure in the southwestern Okinawa Trough? How does it relate to the regional tectonics?

\subsection{Slow slip event}

Megathrust earthquakes and tsunamis induced by rapid rupture along the interface between a subducting oceanic plate and an overlying continental plate can cause serious disasters. Understanding slip behaviours is important for hazard assessment. In a subduction zone, where two plates collide, the subduction interface is locked if the frictional resistance on the fault is larger than the stress. A locked fault can remain inactive for many years, building up large stress and elastic strain before releasing them in an earthquake. Unlike earthquakes, which slip suddenly over seconds to minutes when frictional resistance is overcome, slow slip events (SSEs) release energy slowly, over days to months, and in some cases, years.

With a high risk of large earthquakes, the Pacific Ring of Fire is also a region of frequent SSEs, including Cascadia, Japan, North Island, New Zealand, southern Mexico (e.g., Schwartz and Rokosky, 2007; Peng and Gomberg, 2010). Table 1.1 compares the SSEs observed in some different subduction zones. SSEs in the northern Hikurangi margin has the advantages of shallow $(<10 \mathrm{~km})$, short-lived $(<1$ month), and frequent occurrence ( $<1$ year) to be observed. The subducting oceanic crust in the northern Hikurangi margin is slightly thickened (12 km). Thickened subducting crust has higher buoyancy and shallow dipping subduction interface (Herath et al., 2020; Gase et al., 2021) and therefore the northern Hikurangi margin can hold shallow SSEs. 
Table 1.1: Comparison of SSEs in different subduction zones. Here 'Deep' refers to $>25 \mathrm{~km}$; 'Shallow' refers to $<10 \mathrm{~km}$; 'long-lived' refers to $>1$ year; 'short-lived' refers to $<1$ month; 'infrequent' refers to $>5$ years; and 'frequent' refers to $<1$ year.

\begin{tabular}{llll}
\hline & Depth & Duration & frequency \\
\hline Kapiti & Deep & long-lived & infrequent \\
Manawatu & Deep & long-lived & infrequent \\
Mexico & Deep & long-lived & infrequent \\
Bungo Channel & Deep & long-lived & infrequent \\
Alaska & Deep & long-lived & infrequent \\
southwest Japan & Deep & short-lived & frequent \\
Cascadia & Deep & short-lived & frequent \\
eastern Taiwan & Deep & short-lived & infrequent \\
northern Hikurangi & Shallow & short-lived & frequent \\
\hline
\end{tabular}

Understanding the mechanism of SSEs is particularly important from an earthquake hazard perspective. Some argue that large earthquakes are less likely to happen in the same region where SSEs occur because the elastic strain is released by SSEs (Obara and Kato, 2016). An SSE in Cascadia in 1999 and an SSE in Tokai from 2000 to 2005 released the energy equivalent to an Mw 6.7 earthquake (Dragert et al., 2001) and an Mw 7.1 earthquake (Suito and Ozawa, 2009), respectively. In contrast, Mazzotti and Adams (2004) proposed SSEs may trigger large megathrust events. The occurrence of SSEs is likely to be relevant to earthquakes as slow earthquakes affect the stress in the subduction zone. The physical process of SSEs and the relation to large earthquakes still require further studies.

SSEs and large megathrust earthquakes may have common slip mechanisms, and SSEs can occur next to the spots where earthquakes happen (Figure 1.1; Obara and Kato, 2016). In locked zones, the two plates are strongly coupled, and occasionally the slip occurs suddenly and creates big earthquakes. In contrast, in creeping zones, the slip occurs slowly and produces small earthquakes (Gao and Wang, 2014). SSEs provide evidence for the existence of a transition zone between locked and creeping zones (Hyndman et al., 1995; Dragert et al., 2001; Ohta et al., 2006; Bassett et al., 2014), with the partial release of strain. Thus the study of SSE behaviour may lead us to understand the mechanisms of megathrust earthquakes better. 


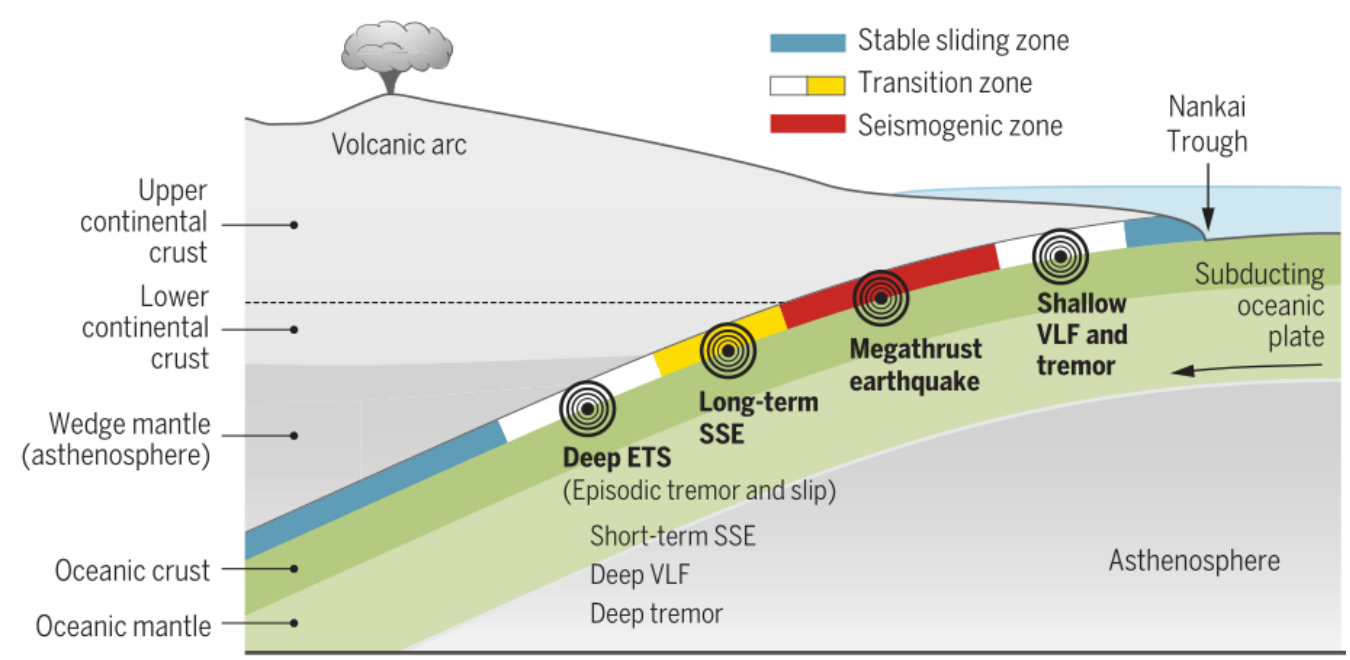

Figure 1.1: The seismogenic zones of earthquakes and SSEs. Abbreviation: SSE, slow slip event; VLF, very low frequency. [From Obara and Kato (2016)]

Audet et al. (2010) suggested that the behavior of SSEs in the transition zone is between aseismic creep and stick-slip, possibly in the presence of high fluid pressures (Wallace et al., 2009). In subduction zones, increased fluid pressure, caused by mineral dehydration and compaction, significantly influences the mechanism of earthquakes and SSEs through its control on effective stress (Saffer and Tobin, 2011; Chaves and Schwartz, 2016).

\subsection{Velocity variations}

Seismic wave velocity, in particular surface wave velocity, is highly related to strain, stress, and fluids, which have all been suggested to be the physical causes of SSEs (Dragert et al., 2001; Kodaira et al., 2004; Warren-Smith et al., 2019). Ambient noise interferometry has been widely used to determine temporal seismic velocity variations (Wegler and Sens-Schönfelder, 2007; Minato et al., 2012; Hobiger et al., 2014; De Plaen et al., 2016; Yates, 2018). Monitoring seismic velocity changes before, during, and after SSEs can help understand SSE behaviour and infer its interactions with strain and stress, or fluids.

Kaproth and Marone (2013) presented simulation work in serpentinite fault zones to study the mechanical origin of slow earthquakes. They found a seismic velocity reduction in the serpentinite before an SSE. Rivet et al. (2011) and Rivet et al. (2014) observed seismic velocity decreases during the SSEs in Mexico in 2006 and 2009 respectively and suggested the velocity decreases were related to the strain rate in 
the overlying crust. Gosselin et al. (2020) observed velocity changes after episodic tremors and slow slip events in Cascadia, which they interpreted to reflect fluctuations in pore fluid pressure.

\subsection{Shear velocity structure}

The velocity of a shear wave $(V s)$ is equal to the square root of the ratio of shear modulus $(G)$, a constant of the medium, to density $(\rho)$ of the medium, $V s=\sqrt{G / \rho}$. Shear velocity, strongly linked to the mediums ability to sustain shear stress and therefore, has a high sensitivity to fluids.

In the past years, Ocean Bottom Seismometers (OBSs) have become widely used to record seismic data (e.g., Webb, 1998, 2007; Webb and Crawford, 2010; Harmon et al., 2007, 2012; Kuo et al., 2009, 2014, 2015; Tian and Ritzwoller, 2015), to observe offshore subduction zones directly and to improve the resolution of the subseafloor velocity structure. The OBSs deployed in southwestern Okinawa Trough can observe the back-arc directly from the seafloor and provide a high-resolution local seismic structure to better understand the opening system and tectonics offshore northeastern Taiwan.

\subsection{Tectonic setting in the Hikurangi margin, New Zealand}

The Pacific Plate subducts beneath the Australian Plate along the Hikurangi margin and moves westwards with a velocity of $45 \mathrm{~mm} / \mathrm{yr}$ (e.g., DeMets et al., 1990, 1994), causing oblique convergence with two subduction zones of opposite polarities. At the southern end of the South Island, the Australian Plate subducts below the Pacific Plate whilst in the North Island the opposite situation occurs with the Pacific Plate being pushed under by the Australian Plate. In between, through most of the South Island, the two plates grind past each other along the Alpine Fault. East of North Island, the Pacific plate subducts beneath the Australian plate along the westward dipping Hikurangi subduction zone (e.g., Walcott, 1984). The northern plate interface is shallow (the shallowest part of the subduction is within $\sim 40 \mathrm{~km}$ of the coastline) compared to many other subduction zones around the world due to its shallow subducting angle $\left(12^{\circ}-25^{\circ}\right)$, which provides an opportunity to observe and study SSEs at a relatively shallow depth $(<15 \mathrm{~km})$ (e.g., Wallace et al., 2004; Douglas et al., 2005). 
The Hikurangi margin can be divided into three segments (Figure 1.2), northern, central, and southern regions. The sediment thickness increases from north to south on the overlying oceanic plate (Lewis et al., 1998). SSEs are shallow, and interseismic coupling is weak in the northern and central segments, while in the southern segment, SSEs are deep, and interseismic coupling is strong (Wallace et al., 2009; Wallace and Beavan, 2010). Wallace et al. (2004) suggested the areas of interseismic coupling are linked to strain accumulation and high stress differences in an elastic medium. The stress accumulation in the strong coupling zone (the southern segment) may lead to earthquakes as the plates are locked. In contrast, the stress accumulation may give rise to SSEs in the weak coupling zones (the northern and central segments) because the stress is distributed more widely on the plate boundary. The northern and central segments are primarily experiencing tectonic erosion (a loss of crust from an overriding tectonic plate due to subduction) and low frontal accretion (the size increase of a tectonic plate by additional material scraped by subduction) compared to the high frontal accretion in the southern segment with its high sediment load (Chanier et al., 1999; Barnes and de Lépinay, 1997; Nicol et al., 2007). The back-arc basins in the northern and central segments are extending, but the back-arc basin in the southern segment is contracting mildly (Stern et al., 1992; Beanland and Haines, 1998; Wallace et al., 2004; Nicol et al., 2007). The accretionary wedge taper angle is 6-10 degrees in the northern and central segments and 4-6 degrees in the southern segment (Barker et al., 2009; Barnes et al., 2010; Wallace et al., 2009).

There are many seamounts in the northern and central segments (Figure 1.2; Bell et al., 2010) but fewer in the southern segment. Some studies show that the seamounts play an important role in tectonic events (e.g., SSEs, tremor and repeating earthquakes; Shaddox and Schwartz, 2019; Todd et al., 2018; Sun et al., 2020). Previous studies suggest that, instead of working as seismic asperities, subducting seamounts may block megathrust earthquakes and facilitate SSEs (e.g., Mochizuki et al., 2008; Wang and Bilek, 2011). A seismic reflection study (Bell et al., 2010) reported high-amplitude reflectivity, suggesting fluid-rich sediments adjacent to the seamounts in the northern and central segments. Based on seismic reflection and magnetic observations, Barker et al. (2018) suggested a 2.5-km-high subducting seamount in the northern segment that was spatially correlated with the September to October 2014 Gisborne SSE, and they supported the view that high fluid 


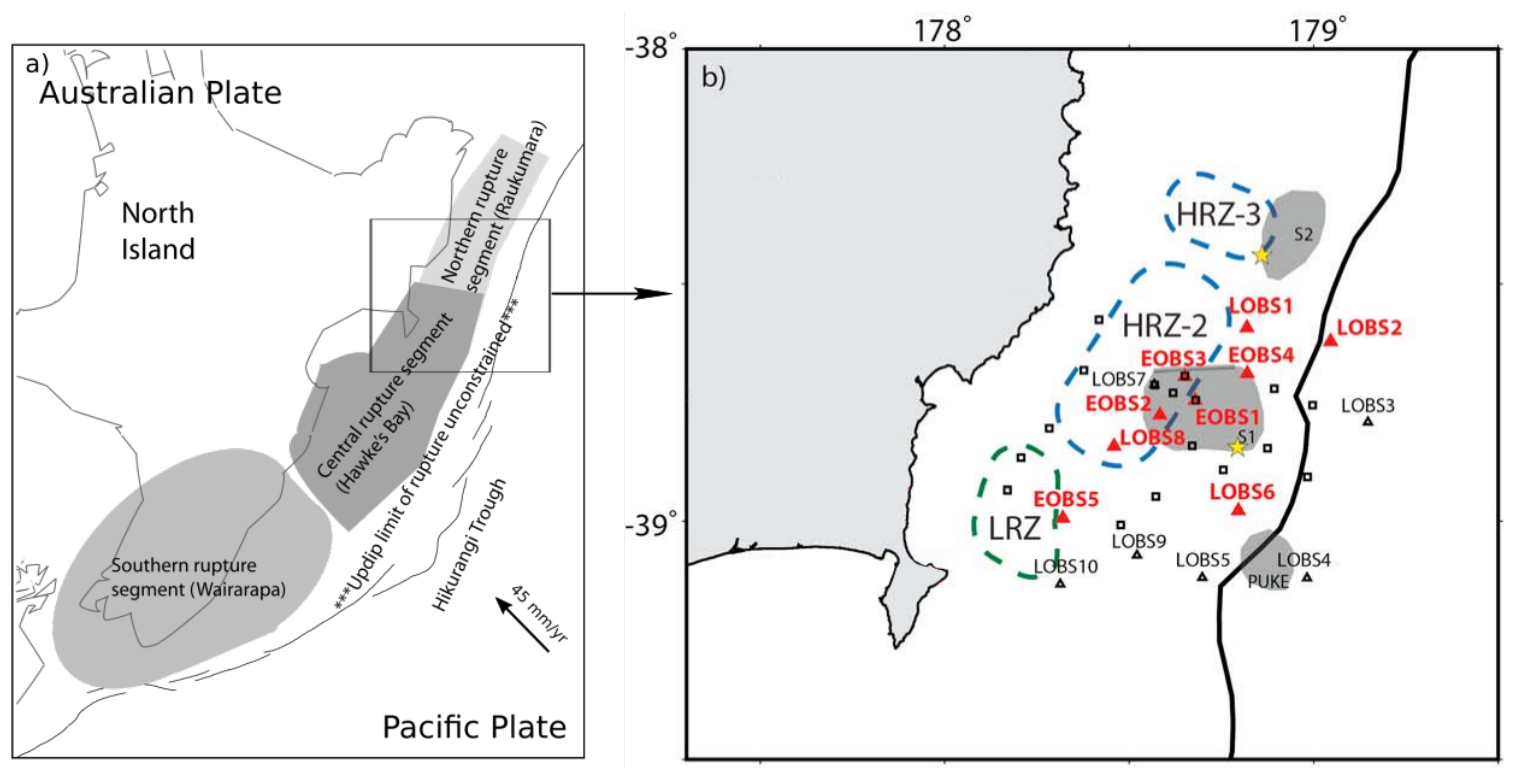

Figure 1.2: Tectonic settings in the Hikurangi margin and seamount distribution. (a) Pacific Plate subducts beneath Australian Plate along Hikurangi Trough, moving westwards with a speed of 45 $\mathrm{mm} / \mathrm{yr}$. Northern, Central, and Southern Segments of the Hikurangi margin are denoted. [From (Wallace et al., 2009)] (b) Seamounts (S1, S2, Puke) are indicated by grey shadows (Bell et al., 2010). High Reflectivity Zones (HRZ-2 and -3) and Lens Reflectivity Zone (LRZ) are marked (Bell et al., 2010). Stations shown in the figure are deployed by the Hikurangi Ocean Bottom Investigation of Tremor and Slow Slip II (HOBITSS II) deployment. [From Todd et al. (2018)]

pressure in the subducting sediment promote shallow SSEs in the northern Hikurangi.

SSEs have been observed in the Hikurangi subduction zone since continuous Global Navigation Satellite System (GNSS) sites started to be installed in 2002 (e.g., Douglas et al., 2005; Schwartz and Rokosky, 2007). SSEs offshore Gisborne have been the focus of several studies because of their short duration (several weeks) and short occurrence intervals (18 to 24 months). Observation from on-land GNSS sites from 2002 (Figure 1.3) involves 1 to $3 \mathrm{~cm}$ horizontal displacement. The SSEs are expected to have larger displacements offshore (Wallace et al., 2016). The frequent occurrence of Gisborne SSEs are ideal for seafloor network deployment as the seafloor instruments can usually only stay on the seafloor for one year due to battery restrictions. Most SSEs are only observed by onshore networks because of the lack of observation offshore. The Hikurangi Ocean Bottom Investigation of Tremor and Slow Slip (HOBITSS) project deployed 24 absolute pressure gauges and 15 OBSs from 2014 to 2015 (HOBITSS II), as well as 18 absolute pressure gauges and 5 OBSs from 2018 to 2019 (HOBITSS V), to expand our observation 
onto the ocean floor. Four main SSEs were captured during the HOBITSS II, and one SSE was captured during the HOBITSS V. In the past, our constraints on SSE slips were limited due to the lack of direct offshore observation. The HOBITSS networks collected data directly above the SSEs, providing better constraints on the spatial distribution of the SSEs. Chapter 3 and Chapter 4 study the velocity variations related to the SSEs in 2014-2015 and 2018-2019, respectively, promoting our understanding of the SSE mechanism.

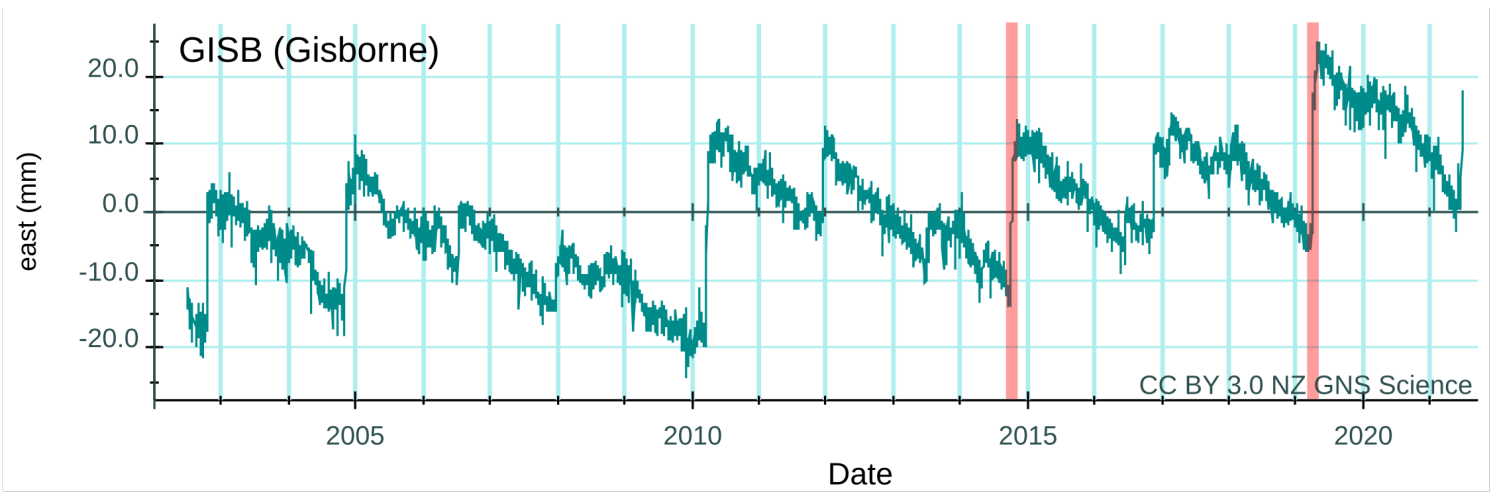

Figure 1.3: SSEs in the northern Hikurangi margin offshore Gisborne recorded by on-land GNSS station GISB from 2002. The x-axis denotes years, and the y-axis denotes east displacement. The jumps in displacement denote SSEs. The shaded red bars highlight the 2014 SSE studied in Chapter 3 and the 2019 SSE studied in Chapter 4. [From GeoNet] 


\subsection{Tectonic setting offshore Taiwan}

Taiwan is formed by the orogeny of the collision of the Eurasian Plate and the Philippine Sea Plate (Figure 1.4). Like New Zealand, the plate-convergent margin in Taiwan comprises two subduction zones of reverse polarities (Wu et al., 2007). In east offshore Taiwan, the Philippine Sea Plate subducts under the Eurasian Plate along the Ryukyu Trench. In contrast, in south offshore Taiwan, the Eurasian Plate subducts under the Philippine Sea Plate along the Manila Trench (Ramsey et al., 2006).

In northeast offshore Taiwan, Ryukyu Arc and Okinawa Trough are formed by the subduction. The southwestern Okinawa Trough is now opening. The southern part of the basin moves south faster than the northward movement of the northern part (Lin et al., 2009). Microearthquakes observed by 15 OBSs deployed during 2003 (Lin et al., 2009) are distributed along normal faults (Sibuet et al., 1998; Sibuet and $\mathrm{Hsu}, 2004)$, indicating normal faults are active in the southwestern Okinawa Trough. There is a back-arc volcanic trail along the back of the Ryukyu arc. High volcanic activity and high heat flow values lead to a low $Q$, high attenuation structure for seismic waves in the Okinawa Trough (Kimura et al., 1986; Tsai et al., 1998; Shyu and Liu, 2001).

Over the last decade, both onshore and offshore seismic networks have been built for understanding the tectonics and hazard assessment offshore Taiwan (e.g., KuoChen et al., 2012; Huang et al., 2014). Kuo-Yen et al. (2005) discussed a $410 \mathrm{~m}$ drill hole Ocean Drilling Program (ODP) Site 1202 (Salisbury, 2002) at $24^{\circ} 48.24^{\prime} \mathrm{N}$, $122^{\circ} 30.00^{\prime} \mathrm{E}$ in southwestern Okinawa Trough drilled at a water depth of $1274 \mathrm{~m}$ (Figure 1.5). The sedimentation rate is significant at this site, ranging from 1.5 to 16 meters per thousand years (Kuo-Yen et al., 2005). Kuo et al. (2015) determined three $1 \mathrm{D}$ shear wave velocity models at three different sites in the Okinawa Trough based on seafloor compliance analysis (Figure 1.5).

In this thesis, we use OBS continuous data to determine the shear wave velocity structure in the southwestern Okinawa Trough to analyse the difference of the two sides of the back-arc rifting axis and provide information on the back-arc tectonics. 


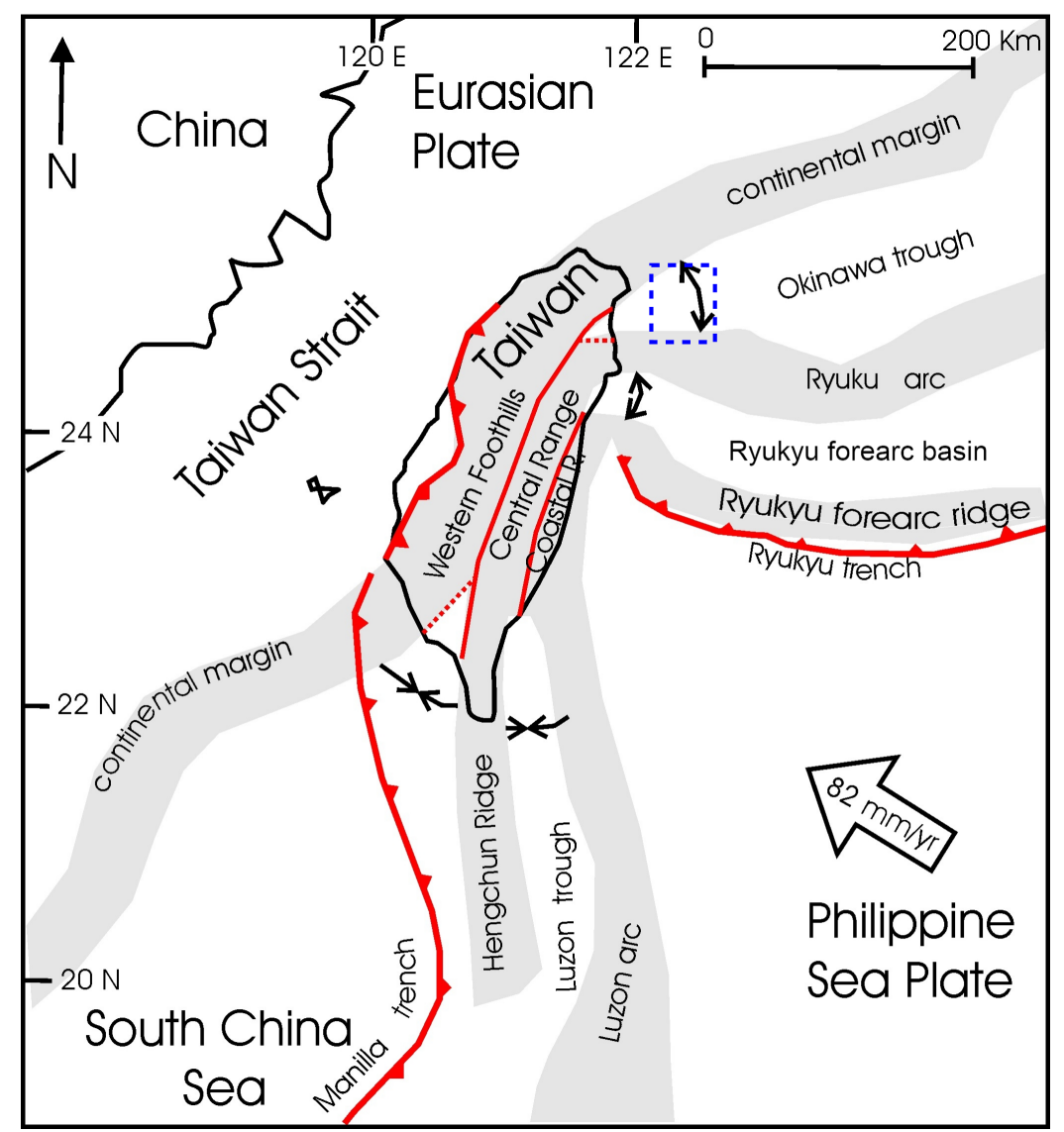

Figure 1.4: Taiwan Tectonic settings. Philippine Sea Plate subducts beneath Eurasian Plate along Ryukyu trench, forming Okinawa trough, Ryukyu arc, and Ryukyu forearc ridge. In contrast, the Eurasian Plate subducts beneath the Phillippine Sea Plate along the Manilla trench, forming Hengchun Ridge, Luzon trough, and Luzon arc. Okinawa trough and Ryukyu forearc basin are extending, while Luzon trough is contracting. The Philippine Sea Plate moves towards the northwest with a velocity of $82 \mathrm{~mm} / \mathrm{yr}$. The blue rectangle marks the study region in this thesis. [From https://www.earthscope.org/about/archived_events/m6.html] 

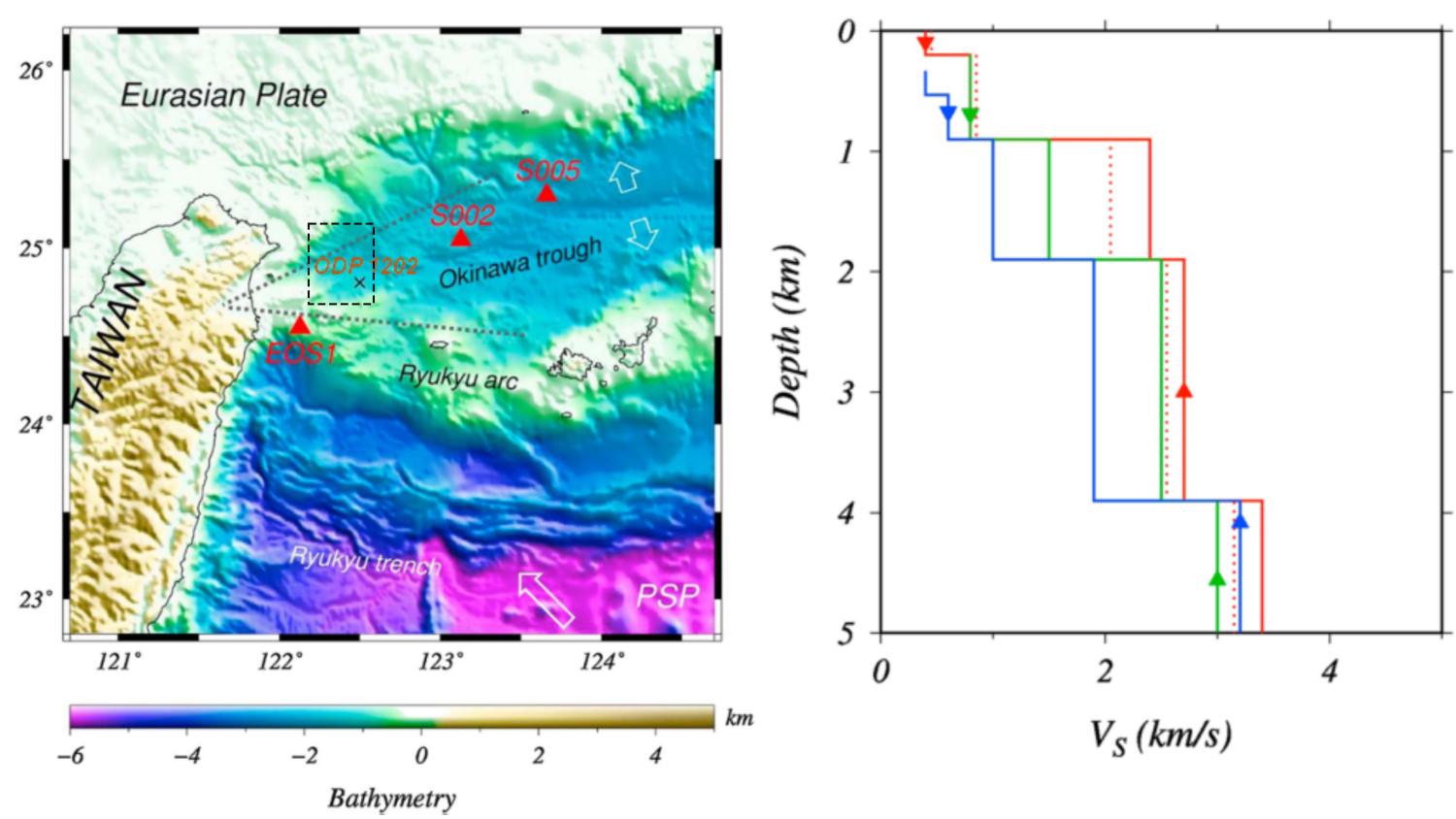

Figure 1.5: Shear wave velocity models in the southwestern Okinawa Trough constrained by seafloor compliance. The left figure shows the locations of three OBSs denoted by red triangles. The black rectangle denotes the study region in this thesis. The cross marks the location of the drilling Ocean Drilling Program (ODP) Site 1202 (Salisbury, 2002). In the right figure, red, green, and blue lines show the shear wave velocity models at EOS1, S002, and S005. [From Kuo et al. (2015)] 


\subsection{Comparison of the Hikurangi and Taiwan margins}

Both Taiwan and New Zealand comprise two subduction zones of reverse polarities (Wu et al., 2007). Table 1.2 summarizes a tectonic comparison of the two study regions. The relative plate motion between the Philippine Sea and the Eurasian plates at Ryukyu Trench is about $80 \mathrm{~mm} / \mathrm{yr}$ (Wu et al., 2007), while the convergence rate at the northern Hikurangi subduction zone is $50 \mathrm{~mm} / \mathrm{yr}$ (Wallace et al., 2004).

Geologically, northern and central Taiwan are underlain by continental crust whereas the southern part corresponds to the transition zone between continent and ocean lithospheres. The southern Okinawa Trough is underlain by a thinned continental crust (about 10 km; Wang et al., 2020). The subducting Pacific Plate at the Hikurangi margin contains the Hikurangi Plateau, an oceanic large igneous province (Coffin and Eldholm, 1994). The Hikurangi plateau is an oceanic large igneous province, where the crustal thickness is about $50 \%$ greater than normal oceanic crust (Mudiyanselage and Herath, 2021). Regular oceanic crust is $7 \mathrm{~km}$ thick while the subducting plate contains the Hikurangi Plateau which has a crustal thickness of 10-12 km (Herath et al., 2020).

Table 1.2: Tectonic and data comparison of southwestern Okinawa Trough and the Hikurangi study regions.

\begin{tabular}{|l|l|l|}
\hline & $\begin{array}{l}\text { Southwestern Okinawa } \\
\text { Trough }\end{array}$ & Northern Hikurangi (NZ) \\
\hline Plate motion & $80 \mathrm{~mm} / \mathrm{yr}$ (Ryukyu Trench) & $50 \mathrm{~mm} / \mathrm{yr}$ \\
\hline Tectonics & reverse polarities & reverse polarities \\
\hline Crust & $\begin{array}{l}\text { thinned continental crust }(10 \\
\mathrm{km})\end{array}$ & $\begin{array}{l}\mathrm{km}) \\
\mathrm{km})\end{array}$ \\
\hline
\end{tabular}

The southwestern Okinawa Trough is actively extending within continental lithosphere (Sibuet et al., 1998), while the Central Volcanic Region of New Zealand is an active back-arc basin developed within continental lithosphere which provides a rare opportunity to study back-arc extension from land-based observations (Stern, 1987). An asymmetric heat output with a particularly high heat flow $\left(800 \mathrm{~mW} / \mathrm{m}^{2}\right)$ in the east of the Central Volcanic Region is suggested to be a consequence of the back-arc asymmetric spreading (Stern, 1987; Stern and Benson, 2011), which is 
supported by the fact that the lithosphere in the east of the Taupo Volcanic Zone is thinner than the west due to the subduction (Stern and Benson, 2011). The asymmetric spreading is considered to be caused by the combination of kinematic and stress conditions that prevail within the central North Island. In the southwestern Okinawa Trough, heat flow measurements (about $110 \mathrm{~mW} / \mathrm{m}^{2}$ on average, varying from 9 to $437 \mathrm{~mW} / \mathrm{m}^{2}$; Shyu and Liu, 2001) are high and are related to continental rifting and seafloor spreading (Liu et al., 2016; Chen et al., 2020). Unlike the Central Volcanic Region of New Zealand, the southwestern Okinawa Trough is lack of seismometer deployment to provide us a high resolution lithosphere depth to investigate if the depths on the two sides of the rifting axis are different.

Table 1.3 compares southwestern Okinawa Trough and Central Volcanic Region. Both areas are in the initial stage of rifting (Miki et al., 1990). Both basins have rotated clockwise by more than $15^{\circ}$, forming wedge-shaped opening (Figure 1.6). The wedge-shaped opening may be the initial stage of the fan-shaped opening of a mature basin, such as the opening of the Japan Sea (Otofuji and Matsuda, 1987) north of the Okinawa Trough.

Table 1.3: Comparison of Southwestern Okinawa Trough (Taiwan) and Central Volcanic Region (NZ).

\begin{tabular}{|l|l|l|}
\hline & $\begin{array}{l}\text { Southwestern Okinawa } \\
\text { Trough }\end{array}$ & $\begin{array}{l}\text { Central Volcanic Region } \\
(\mathrm{NZ}))\end{array}$ \\
\hline Volcano & 2 - 0 Ma & 4 - $0 \mathrm{Ma}$ \\
\hline Back-arc extension & $\begin{array}{l}\text { actively extending (ini- } \\
\text { tial stage) }\end{array}$ & $\begin{array}{l}\text { actively extending (initial } \\
\text { stage) }\end{array}$ \\
\hline Asymmetric extension \\
evidence & $\begin{array}{l}\text { hedge-shaped opening } \\
\left(10^{3} m W / m^{2}\right) \text { in south }\end{array}$ & $\begin{array}{l}\text { wedge-shaped opening } \\
\left(800 m W / m^{2}\right) \text { in the east }\end{array}$ \\
\hline & $\begin{array}{l}\text { hening may occur only } \\
\text { in the south in } 1.9-0.5 \\
\text { Ma } 0\end{array}$ & \\
\hline
\end{tabular}




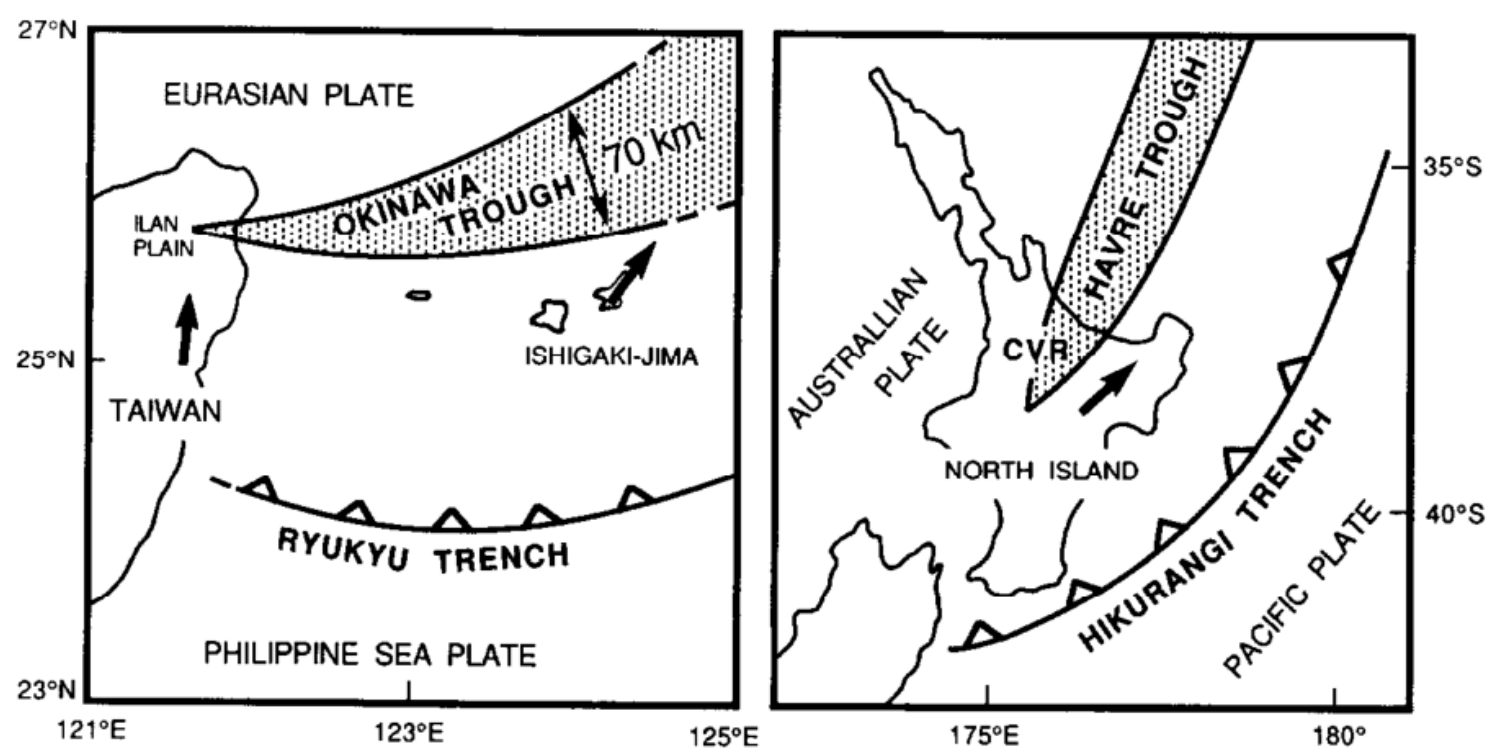

Figure 1.6: Wedge-shaped opening of the Okinawa Trough and the Central Volcanic Region of New Zealand. Ryukyu Arc and the eastern North Island of New Zealand have been rotated clockwise in association with the wedge-shaped back-arc spreading. [From Miki et al. (1990)]

\subsection{Thesis structure}

This thesis includes six chapters. Chapter 1 introduces the study background, including study topics and study region tectonics. Chapter 2 describes the data and methods used in this thesis. Chapters 3, 4, and 5 are the main body of this thesis, representing three research projects. Each project has individual abstract, data, method, result, discussion, and conclusion sessions. Chapter 3 was submitted to the journal "Earth and Planetary Science Letters" on 14th March 2021. Chapter 6 synthesizes the outcomes of these three projects, and their relation to each other.

\section{Chapter 1: Introduction}

Chapter 1 introduces slow slip events (SSEs) and seismic velocity variations studied in Chapters 3 and 4, as well as back-arc extension/rifting and shear wave velocity structure studied in Chapter 5. Tectonic settings of the study regions of Chapters 3 and 4 (New Zealand) and Chapter 5 (Taiwan) are also introduced.

\section{Chapter 2: Data and methodology}

Chapter 2 introduces the data and methods used in Chapters 3, 4, and 5. Data include 20 Ocean Bottom Seismometers (OBSs) offshore northeastern New Zealand 
and 34 OBSs offshore northeastern Taiwan. Methods include ambient noise (used in all three chapters), velocity variations (used in Chapters 3 and 4), phase velocity measurement (used in Chapter 5), and shear wave inversion (used in Chapter 5).

\section{Chapter 3: Temporal velocity variations in the northern Hikurangi margin and the relation to slow slip}

Chapter 3 investigates the temporal seismic velocity variations related to slow slip events (SSEs) in the northern Hikurangi Margin (offshore Gisborne, New Zealand) during the HOBITSS II in 2014-2015. 15 OBSs deployed by the HOBITSS II are used in this chapter. Four main SSEs occurred during the deployment, with the biggest SSE having a significant slip over $250 \mathrm{~mm}$ beneath the HOBITSS II deployment. This chapter studies the velocity variations related to the four SSEs, particularly the biggest SSE. We find that the velocity decreases during the SSE and increases after the SSE back to the original value. Our study demonstrates that velocity variations related to SSEs are detectable and provides evidence to support some hypotheses of the SSE mechanism.

This chapter is submitted to Earth and Planetary Science Letters as "Temporal velocity variations in the northern Hikurangi margin and the relation to slow slip". Weiwei Wang is the first and corresponding author. Weiwei Wang leads the project of this chapter and conducts the main work, including data processing, methodology, visualization, interpretation, and writing. Co-authors include Martha K. Savage, Alec Yates, Hubert J. Zal, Spahr Webb, Carolyn Boulton, Emily WarrenSmith, Megan Kortink, Tim Stern, Bill Fry, Kimihiro Mochizuki, and Laura Wallace. Continuous seismic data used came from Spahr Webb (Lamont-Doherty Earth Observatory, Columbia University) and Kimihiro Mochizuki (Earthquake Research Institute, University of Tokyo). Martha K. Savage provides supervision to the work of Weiwei Wang and contributes to funding acquisition. Alec Yates provides technical support on methodology and paper editing. Hubert J. Zal contributes to interpretation and visualization. Spahr Webb contributes to data collection, methodology, paper editing, and funding acquisition. Carolyn Boulton and Emily Warren-Smith contribute to interpretation and paper editing. Megan Kortink supports the methodology. Tim Stern and Bill Fry provide supervision and interpretation suggestions. Kimihiro Mochizuki contributes to data collection, paper editing, and funding acquisition. Laura Wallace contributes to paper edit- 
ing, interpretation, and funding acquisition. Except for the co-authors mentioned above, Pasan Herath and Katherine Woods kindly provided suggestions for the Hikuangi tectonics and slow slip events.

Supplemental materials for this chapter can be found in Appendix A.

\section{Chapter 4: Temporal velocity variations in 2018-2019 in the northern Hikurangi margin and the relation to slow slip}

Based on the process in Chapter 3, this chapter follows the same workflow and applies the same methods to another network deployed in the same region. 5 OBSs deployed by the HOBITSS V (2018-2019) are used in this chapter. One SSE occurred during the HOBITSS V deployment but with a smaller slip than the biggest SSE in 2014 (Chapter 3). The main slip was in the south of the deployment, and the slip beneath the deployment was up to $150 \mathrm{~mm}$. Similar to what was found in Chapter 3, the velocity decreases during the SSE and increases after the SSE, but they are smaller than the variations in Chapter 3. This chapter provides more evidence to support the hypotheses in Chapter 3. Supplemental materials for this chapter can be found in Appendix B.

This chapter was written in collaboration with Martha K. Savage and Kimihiro Mochizuki. Continuous seismic data came from Kimihiro Mochizuki (Earthquake Research Institute, University of Tokyo). Weiwei Wang undertakes the project and conducts the main work, including data collection, data processing, methodology, visualization, interpretation, and writing. Martha K. Savage provides supervision to the work of Weiwei Wang and contributes to funding acquisition. Kimihiro Mochizuki contributes to data collection and funding acquisition.

\section{Chapter 5: Studies of shear wave velocity structure in the southwestern Oki- nawa Trough from continuous OBS data}

Chapter 5 focuses on the shear wave velocity structure and the tectonics in the southwestern Okinawa Trough offshore northeastern Taiwan. For instrument development and tectonic study, Academia Sinica deployed 34 OBSs at various time periods between 2010 to 2018. This study uses the ambient noise data recorded on the OBSs to study the shear wave velocity structure in the southwestern Okinawa Trough. We find the velocity in the south of the back-arc rifting axis and near the 
axis is slower than the velocity in the north. This could be caused by a high heat flow in the south of the rifting axis, which may result in asymmetric back-arc extension/rifting. In addition to the velocity model, this study also suggests effective techniques for OBS noise correction and unwrapping the cycle skipping of phase velocity measurements. Supplemental materials for this chapter can be found in Appendix C.

This chapter was written in collaboration with Shu-Huei Hung, Martha K. Savage, Yinhe Luo, Xiaozhou Yang, Hao Rao, Pei-Ying Patty Lin, Spahr Webb, Ban-Yuan Kuo, Tim Stern, Bill Fry, and Hsin-Ying Yang. Weiwei Wang undertakes the project and conducts the main work, including data processing, methodology, visualization, interpretation, and writing. Shu-Huei Hung contributes to data collection, data processing, and methodology. Martha K. Savage contributes to data processing, interpretation, editing, and supervision. Yinhe Luo, Xiaozhou Yang, and Hao Rao contribute to data processing and methodology. Pei-Ying Patty Lin, Spahr Webb, and Hsin-Ying Yang contribute to methodology. Ban-Yuan Kuo contributes to data collection and funding acquisition. Tim Stern contributes to interpretation. Bill Fry contributes to data processing.

\section{Chapter 6: Conclusions}

Chapter 6 synthesizes the findings of the three projects (Chapters 3, 4, and 5) and suggests avenues for future study. 


\section{Chapter 2}

\section{Data and methodology}

\subsection{Data acquisition}

The data for this thesis were obtained from several sources, including temporary ocean bottom instrument deployments and the permanent GeoNet network. This thesis mainly uses ambient noise data recorded by Ocean Bottom Seismometers (OBSs): two networks offshore eastern New Zealand and one network offshore northeastern Taiwan. More information can be found in corresponding chapters.

\subsubsection{HOBITSS}

The Hikurangi Ocean Bottom Investigation of Slow Slip (HOBITSS) project was part of a project funded by the National Science Foundation (NSF), the Earthquake Research Institute (ERI), University of Tokyo, and New Zealand's GeoNet project. From May 2014 to June 2015, ten Lamont-Doherty broadband Ocean Bottom Seismometers (LOBS) and five Earthquake Research Institute, University of Tokyo short period Ocean Bottom Seismometers (EOBS) with a natural period of $1 \mathrm{~s}$ were deployed (Figure 2.1). Four slow slip events (SSEs) occurred during the deployment, and two of them had slip under the OBS deployment. More information can be found in Chapter 3. Raw data from this deployment were downloaded from the Incorporate Research Institutions for Seismology Data Management Center (IRIS-DMC) with network code YH (2014-2015). Continuous seismic data were downloaded in miniseed format. The data continuity is good, except for EOBS5 
lost about one month of data for November 2014.

From October 2018 to October 2019, five EOBSs were deployed (Figure 2.1). The instrument type is the same as the EOBSs deployed in 2014-2015. During the deployment, one SSE occurred with some of its slip under the OBS deployment. More information can be found in Chapter 4. Raw data were extracted and pre-processed by Earthquake Research Institute (ERI), University of Tokyo, and copied to us. The data continuity is good.

The OBSs are equipped with three components - one vertical and two horizontal seismic channels. All the three components are used in this thesis (Chapters 3 and $4)$.

\subsubsection{Southwestern Okinawa Trough deployment}

From 2010 to 2017, for the development of OBS production, the Institute of Earth Sciences, Taiwan (IES) deployed 34 OBSs during different times (Figure 2.2) in southwestern Okinawa Trough, from which five IES OBSs deployed in 2010 are with a natural period of 3 s, three IES OBSs deployed in 2011 are with a natural period of $7 \mathrm{~s}$, twenty-one IES OBSs deployed at various times from 2015 to 2018 are broadband, and five Germany OBSs deployed in 2011 are broadband. More information can be found in Chapter 5. Except for the three component seismic channels, most of the OBSs are equipped with one more differential pressure gauge (Figure 2.2). Raw data used in this study were copied from IES in miniseed format. The data continuity is good. The seismic vertical channels and differential pressure gauges are used for analysis.

\subsubsection{GNSS}

The Global Navigation Satellite System (GNSS) network is a part of GeoNet. The majority of ground deformation data are collected continuously using GNSS receivers and antennas. GeoNet (http://www.geonet.org.nz) is a project to build and operate a modern geological hazard monitoring system in New Zealand. GNSS network is funded by the Earthquake Commission (EQC). It is designed and maintained by GNS Science and Land Information New Zealand (LINZ).

To constrain the timing of the SSE in 2019, three GNSS stations close to the HO- 
BITSS V deployment are used in this study, providing time-series ground information caused by the SSE. Details can be found in Chapter 4.

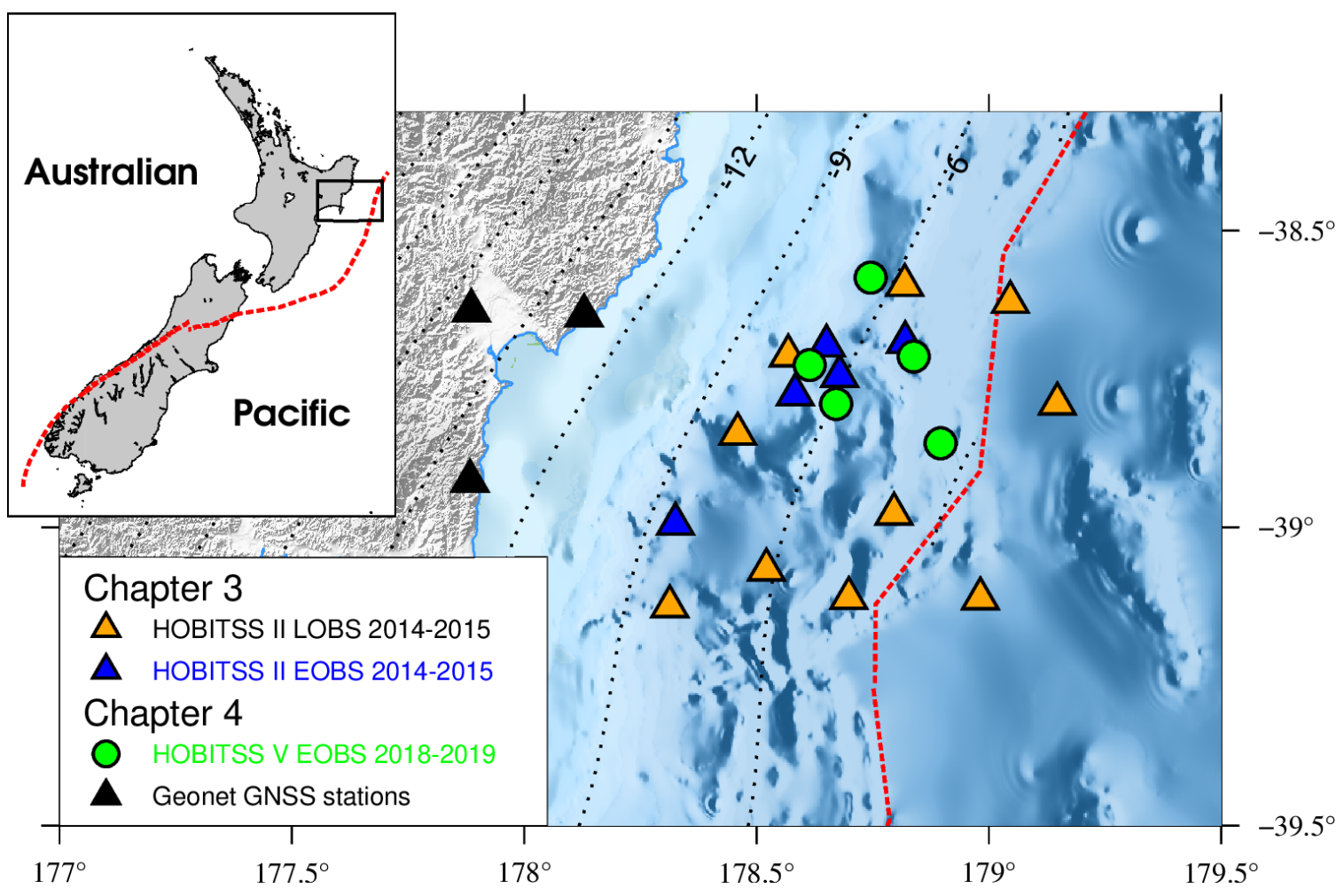

Figure 2.1: Stations used in Chapter 3 and Chapter 4. The orange triangles denote the LOBSs and the blue triangles denote the EOBSs deployed by HOBITSS II in 2014-2015, which are used in Chapter 3. The green circles denote the EOBSs deployed by HOBITSS V in 2018-2019 and the black triangles denote permanent GNSS stations deployed by Geonet, used in Chapter 4. 


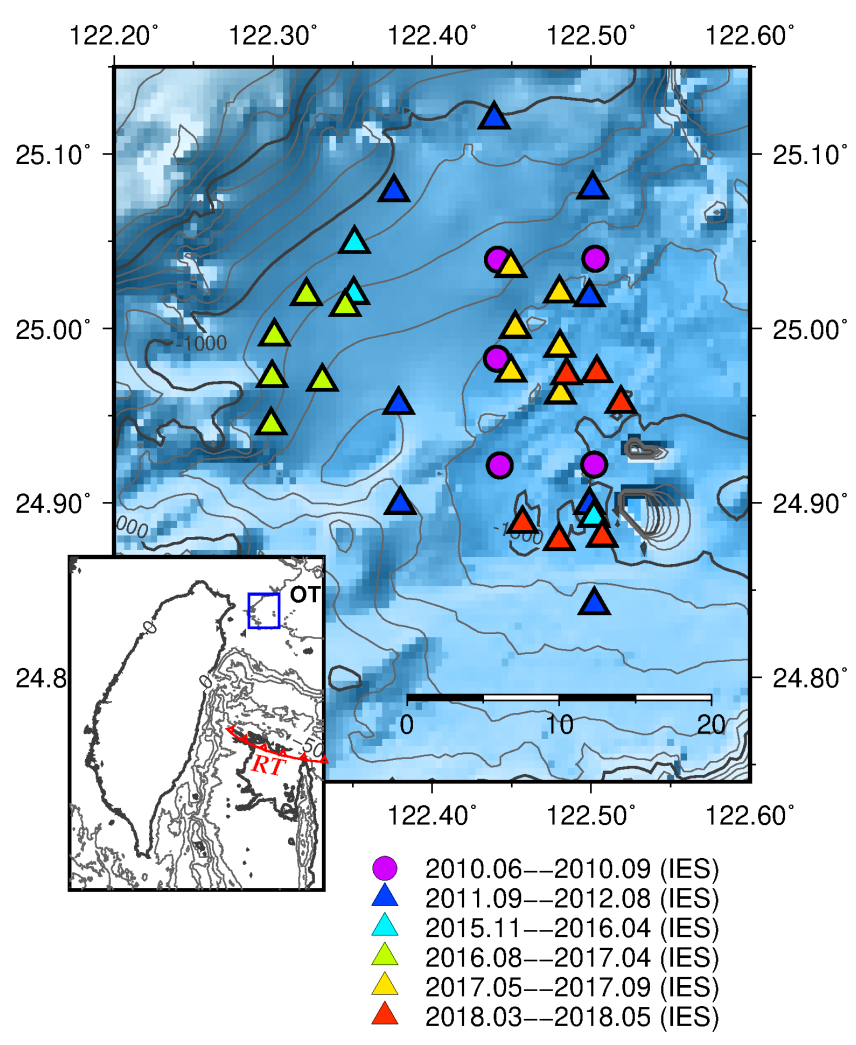

Figure 2.2: Stations used in Chapter 5. Different colors of stations denote the OBSs deployed at different times. Circles indicate the instruments equipped with 3-component seismometers only, triangles indicate the instruments equipped with differential pressure gauges and 3-component seismometers. 


\subsection{Ambient noise}

In the last 20 years, seismic ambient noise has been widely used to study the crust and upper mantle shear velocity structure (e.g., Shapiro and Campillo, 2004; Harmon et al., 2007; Yang et al., 2020), as well as velocity variations related to earthquakes (e.g., Brenguier et al., 2008; Minato et al., 2012; Froment et al., 2013), volcanic eruptions (e.g., Sens-Schönfelder and Wegler, 2006; Yates, 2018), and slow slip events (Rivet et al., 2011). Seismic interferometry cross-correlates continuous background noise recorded at two stations to construct surface waves traveling along the station path, which removes the reliance on the occurrence of earthquakes. Campillo and Paul (2003) first suggested that the impulse response of the Earth, particularly surface wave signals excited from a single point-force source, could be retrieved by cross-correlating earthquake coda waves recorded at two stations. The retrieved signals are often referred to as empirical Green's functions. Shapiro and Campillo (2004) and Shapiro et al. (2005) recognized that ambient noise can be considered as a diffuse wavefield because of its random distribution. They further demonstrated that Rayleigh wave signals can be retrieved by stacking the inter-station cross-correlation functions (CCFs) of continuous ambient noise over long time periods. The resulting waveforms and frequency-dependent group and phase velocities measured from the waveforms are compatible with those extracted from earthquakes. As the noise records are not restricted to earthquake distributions, the CCF-extracted surface waves have provided unprecedentedly dense inter-station path coverage, which has been widely used in surface wave group and phase velocity tomography for the shear wave velocity structures of the crust and upper mantle, especially in highly instrumented continental regions (e.g., Shapiro et al., 2005; Yao et al., 2006; Yang et al., 2008).

The multiple-scattering theory has validated that for a diffuse field with evenly distributed noise sources, the CCF between two seismic wavefields received at two stations is equivalent to the Earth's response, or Green's function, at one station excited by an impulsive force acting at the other (Lobkis and Weaver, 2001). Such conditions can be achieved by increasing the averaging of the CCFs from uniformly distributed earthquakes or noise sources (Wapenaar et al., 2010). As shown in Figure 2.3, the CCF-extracted Green's function depends on the distribution of noise sources. If the noise sources are evenly distributed, the waveforms of 
the computed CCFs are symmetric with respect to zero time lag (Wapenaar et al., 2010). In reality, evenly distributed noise sources are not common. Since the origins of ambient noise are strongly linked with ocean waves driven by winds and interacting with the coastline and seafloor bathymetry, and human activity, their distribution and spectral characteristics appear to vary over time and region (e.g., Tian and Ritzwoller, 2015; Wang et al., 2016). As such, the retrieved Empirical Green's Functions are in reality far from attaining the condition with evenly distributed noise sources, showing large amplitude asymmetric variations between the positive time lag and negative time lag of the signals (Figure 2.4).

In Figure 2.5, the curves show the upper and lower bounds of noise levels observed in the global seismograph network, obtained by averaging the power spectral density (PSD) of ground acceleration over a large collection of seismic data segments (Peterson et al., 1993). The worldwide ambient noise field has two predominant peaks in the period band between 1 and $20 \mathrm{~s}$, commonly referred to as microseisms. They are caused by the non-linear interaction of ocean waves and seabed bathymetry in coastal zones (Longuet-Higgins, 1950; Cessaro, 1994; Webb, 2007; Gerstoft and Bromirski, 2016). The noise at periods between $10 \mathrm{~s}$ and $20 \mathrm{~s}$ typically peaked around $16 \mathrm{~s}$, is produced through the interaction of ocean waves with coastal seafloor (Hasselmann, 1963) and is termed primary microseism. The noise at periods between 5 and $10 \mathrm{~s}$ with a stronger peak around $6 \mathrm{~s}$ is generated through non-linear interference of incoming and reflected ocean waves (Longuet-Higgins, 1950), having double the frequency of ocean waves, and is called secondary microseism. Primary microseism appears to be generated only in shallow water from the conversion of water waves into seismic waves of the same frequency through pressure variations at the seafloor (Hasselmann, 1963). Secondary microseism can be generated in shallow and deep water by the interference of two waves with equal frequencies moving in opposite directions (Longuet-Higgins, 1950). This interference generates standing waves with small attenuation with water depth, which can excite surface waves on the ocean floor (Longuet-Higgins, 1950). Ambient noise at higher frequencies of $1-10 \mathrm{~Hz}$, commonly known as microtremor, is often dominated by cultural sources such as vehicular traffic (e.g., Wang et al., 2016).

In this thesis, Chapter 5 applies ambient noise interferometry on station pairs, while Chapter 3 and Chapter 4 compute single station cross-component corre- 
lations. Wegler and Sens-Schönfelder (2007); Minato et al. (2012); Hobiger et al. (2014); De Plaen et al. (2016); Yates (2018) have applied single station cross-correlations for velocity change monitoring. Single station cross-component correlations assume a pulse excited at one component traveling out and are scattered back, received by another component. The application of seismic interferometry in velocity variation monitoring is based on the sensitivity of surface waves to small perturbations in the elastic properties of the crust. Elastic moduli $\lambda$ and $\mu$ are used to describe the properties of an isotropic material. Compressional wave velocity $\alpha$ and shear wave velocity $\beta$ are

$$
\alpha=\sqrt{\frac{\lambda+2 \mu}{\rho}}, \beta=\sqrt{\frac{\mu}{\rho}}
$$

where $\rho$ is density. Assuming a constant density, a change of elastic moduli would lead to a velocity change.
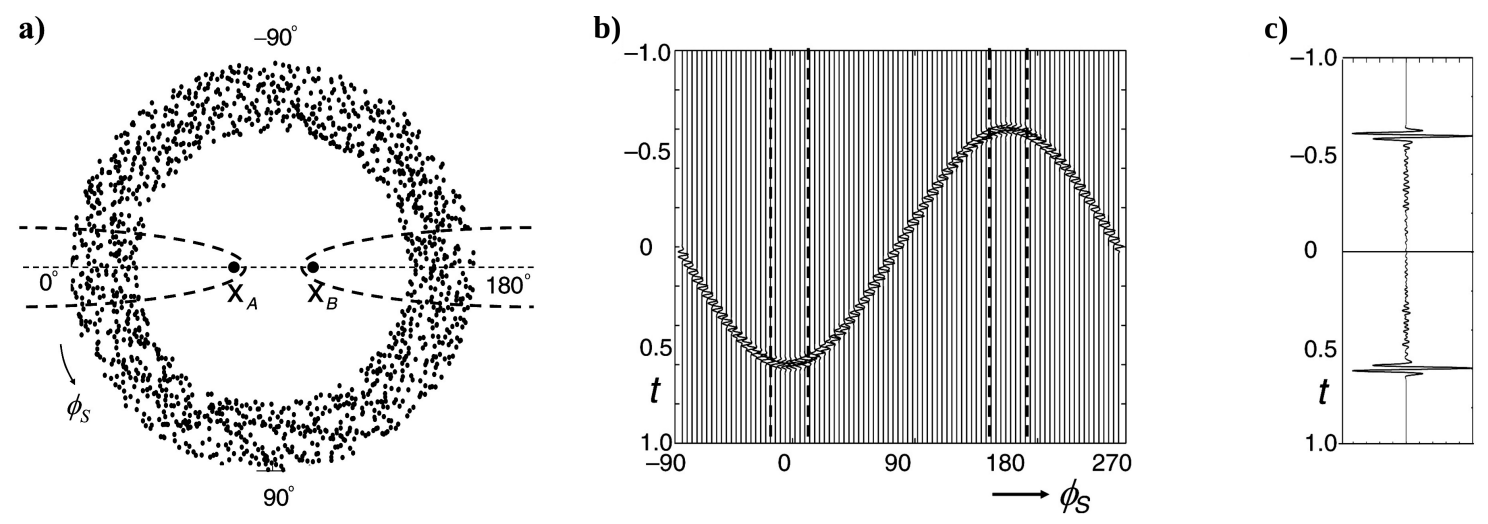

Figure 2.3: 2-D illustration of ambient noise interferometry. (a) Configuration of point sources (small dots) and two stations $X_{A}$ and $X_{B}$, receiving the signals emitted from the sources, at azimuth $\phi_{s}$, measured counterclockwise from the left to the right of the line. The $\phi_{s}$ is equally-spaced at an interval of $0.25^{\circ}$. The dashed curves denote the Fresnel zones. The medium is assumed to be uniform with no energy loss during the wave propagation. (b) Cross-Correlation Functions (CCFs) of the responses at $X_{A}$ and $X_{B}$ excited from the point sources at the same azimuth. The arrival times of the cross-correlated signals vary smoothly with $\phi_{s}$, depending only on the arrival-time difference between the signals at $X_{A}$ and $X_{B}$. The CCFs emitted from the sources within the Fresnel zones are bounded by vertical dashed lines. (c) The stack of the CCFs in (b) over azimuth. Only the CCFs from the sources within the Fresnel zones shown by dashed curves in (a) interfere constructively, which leads to the large-amplitude symmetric signals in the positive and negative time lags. [From Wapenaar et al. (2010)] 


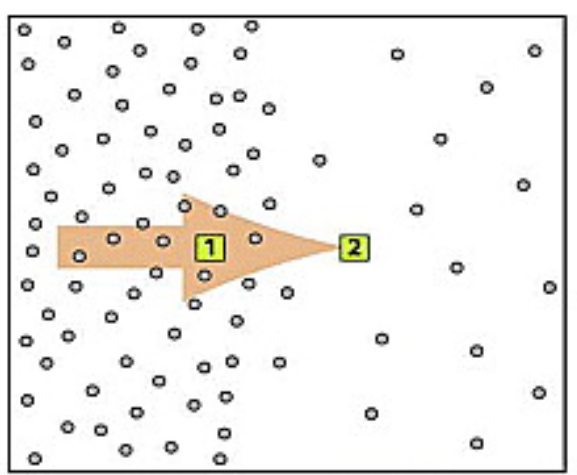

cross-corrleation 1-2

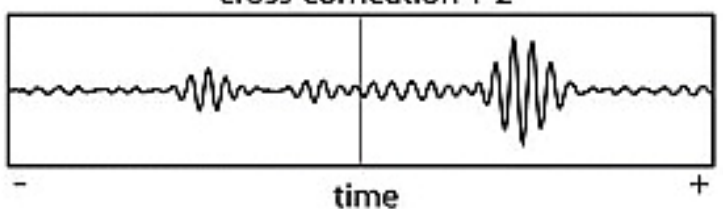

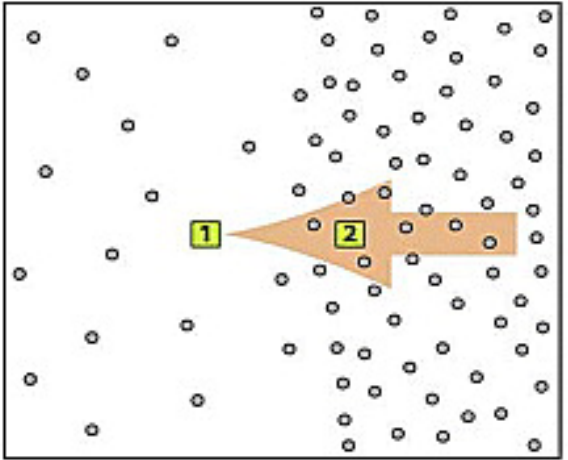

cross-corrleation 1-2

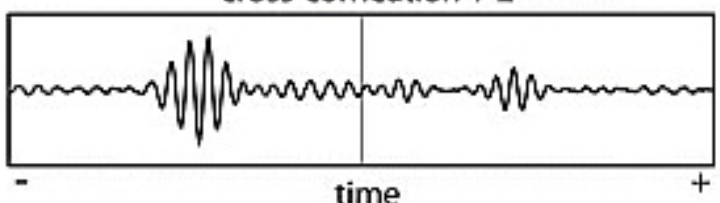

Figure 2.4: Unevenly distributed noise sources and corresponding CCFs. Distribution of noise sources shown on the top and corresponding empirical Green's functions derived from noise crosscorrelations between stations 1 and 2 on the bottom. In the positive lag, the wave is excited at 1 (source) and travels to 2 . In the negative lag, the wave is excited at 2 (source) and travels to 1 . The noise sources are unevenly distributed, dominated in one direction from station 1 to 2 and in the other direction from station 2 to 1, respectively. [From Stehly et al. (2006)].

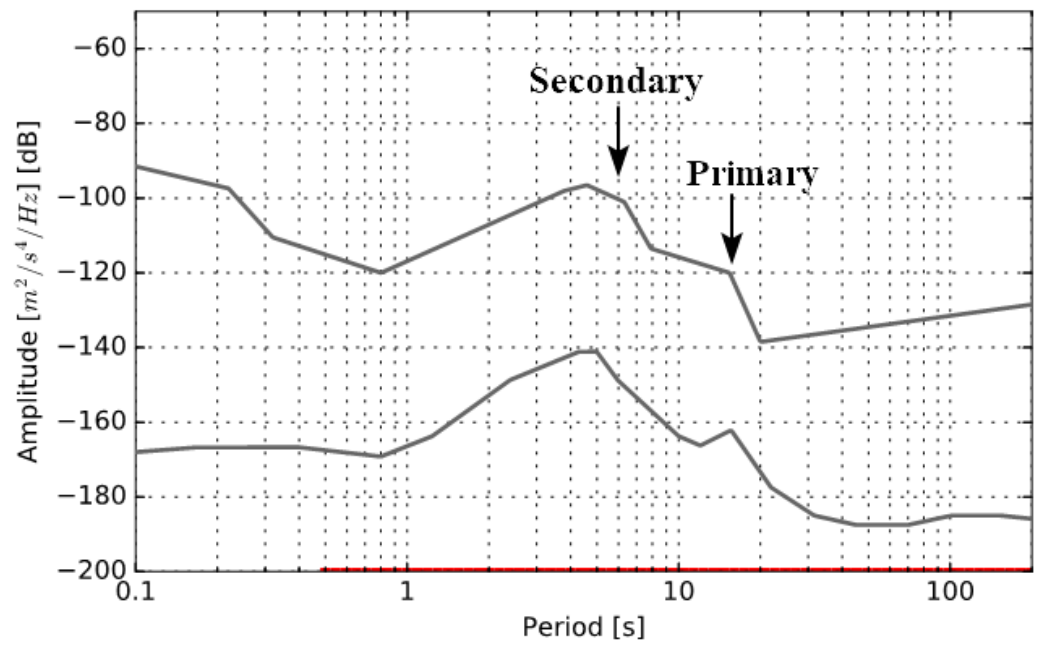

Figure 2.5: Global high noise and low noise models for power spectral densities (PSDs) of ground acceleration, estimated by averaging the PSDs at the global seismograph network (Peterson et al., 1993). The PSD is in decibels (dB) units relative to the metric unit $1\left(\mathrm{~m}^{2} / \mathrm{s}^{4}\right) / H z$. The smaller peak at periods between 10 and $20 \mathrm{~s}$ (typically around $16 \mathrm{~s}$ ) corresponds to the so-called primary microseism generated in shallow water, having the same period as the water waves that produce them. The stronger peak at periods between 5 and $10 \mathrm{~s}$ (typically around $6 \mathrm{~s}$ ) having double the frequency of the generating gravity waves is referred as to the secondary or double frequency microseism, arising from the interaction of ocean waves with equal frequencies moving in opposite directions (Longuet-Higgins, 1950). 


\subsection{MSNoise}

MSNoise (Measuring with Seismic Noise) is a python package that provides a method to go from raw noise data to velocity variations. The process includes waveform pre-processing, cross-correlation computation, stacking, and velocity change measurement. Parameterization is discussed in later chapters. MSNoise was first released in 2014. This study uses MSNoise version 1.5. After version 1.6 was released in 2019 (not used in this study), I developed a shell script package with some database language to use MSNoise, which is available at https: //github.com/wwwWeiweiWang/MSnoise1.6_scripts.

The process to cross-correlate functions follows Bensen et al. (2007). The following process to compute velocity variations is described in Lecocq et al. (2014).

\subsection{Cross-correlation functions}

In this thesis, ambient noise data are used to compute cross-correlation functions (CCFs) to retrieve surface waves. In Chapter 3 and Chapter 4, coda waves are used for velocity variation calculation, while in Chapter 5, direct waves are used for the shear velocity structure. Therefore, in Chapter 3 and Chapter 4, cross-correlations are computed aiming to maximise the Signal-Noise Ratio (SNR) of coda waves, while in Chapter 5, cross-correlations are computed aiming to maximise the SNR of direct waves.

The raw data are one-day miniseed format files. They are transferred to SAC (Seismic Analysis Code) format using mseed2sac software released by IRIS (https: //ds.iris.edu/ds/nodes/dmc/software/downloads/mseed2sac/). To save storage space, the data are down-sampled. For Chapter 3 and 4, there are two types of instruments. The sampling rate of LOBS is $100 \mathrm{~Hz}$, and the sampling rate of EOBS is $200 \mathrm{~Hz}$. In Chapter 5, the sampling rate of the instruments is 100 $\mathrm{Hz}$. All the data are down-sampled to $20 \mathrm{~Hz}$, as the highest frequency this study is interested in is lower than $10 \mathrm{~Hz}$ (Nyquist frequency). The one-day SAC data are then divided into several segments with overlap, such as 7200-second segments with $70 \%$ overlap in window length. The length of segments and value of overlap vary among different datasets, which are discussed in Chapter 3, Chapter 4, and Chapter 5. The instrument responses of the seismic components are only removed 
if the cross-correlations are computed among different instrument types. Crosscorrelation functions are computed following the standard processing procedures described by Bensen et al. (2007) using the python package MSNoise (Lecocq et al., 2014). The data are pre-filtered at a band of $0.02 \mathrm{~Hz}$ to $2 \mathrm{~Hz}$ followed by timedomain normalization and spectral whitening procedures (Bensen et al., 2007) to suppress earthquake signals and instrumental irregularities. There are different methods for time-domain normalization provided by MSNoise: 1-bit and RootMean-Square. 1-bit normalization replaces all the positive amplitude with 1 and all negative amplitude with -1 (Bensen et al., 2007). Root-Mean-Square normalization clips amplitude to an integer multiple of the Root-Mean-Square of the trace (Tukey, 1962; Yates, 2018). In the following process, the data are filtered in a narrower frequency band before cross-correlations are computed in the frequency domain. Assuming two time series $x(t)$ and $y(t)$ and their Fourier transforms $X(f)$ and $Y(f)$, the cross-correlation function between $x(t)$ and $y(t)$ is defined as

$$
C(f)=X^{*}(f) \times Y(f)
$$

$C(f)$ is then transferred back to the time domain. Afterwards, cross-correlation functions computed by the segments within one day are added together (one-day stack), and one-day stacks can be further stacked for different purposes (see Chapters 3,4 , and 5 for details). A general purpose is to obtain stable signals and enhance SNR by constructive interference of the signals and destructive interference of random noise. MSNoise provides two stacking methods: linear stack and phase weighted stack. Linear stack is the mean of the cross-correlations,

$$
c(t)=\frac{1}{n} \sum_{i=1}^{n} s_{i}(t)
$$

where $c(t)$ is the stack, $s_{i}(t)$ is the cross-correlation function, and $n$ is the number of cross-correlation functions. Phase weighted stack adds weighting based on the coherency of instantaneous phases (Schimmel and Paulssen, 1997):

$$
c(t)=\frac{1}{N} \sum_{i=1}^{n} s_{i}(t)\left|\frac{1}{n} \sum_{j=1}^{n} \exp \left[i \phi_{j}(t)\right]\right|^{\kappa}
$$

where $\phi_{j}(t)$ is the phase of one cross-correlation function, and the parameter $\kappa$ controls the emphasis on coherent phases, which is related to the parameter pws_power in MSNoise. Through a phase-weighted stack, every sample of the linear stack is 
weighted by the coherency of its instantaneous phases. Therefore, weak coherent signals can be enhanced through noise reduction. In Chapter 5, the phase weighted stack is applied to emphasize the direct arrivals. In Chapter 3 and 4, the linear stack is applied for scattered coda waves.

The determination of parameters are discussed in Chapter 3, 4, and 5. In Chapter 3 and 4, velocity variations are computed using MSnoise after stacking (Section 2.5). In Chapter 5, all available CCFs are stacked to maximise the SNR of the direct waves. The work with MSNoise in Chapter 5 finishes here. Afterwards, phase velocities are measured from stacks, following by shear velocity inversion (Section 2.6).

\subsection{Velocity variations}

Velocity variations are determined by measuring the time differences between successive current stacks (moving-window stack) and the reference stack (Brenguier et al., 2008; Yates, 2018). The reference stack (e.g., stack of 300 days) represents a background state that is stacked by more days than the current stack, while the current stack (e.g., stack of 20 days) indicates a stack over a short time and will be compared with the reference stack.

The reconstruction of a stable reference stack largely controls the accuracy of the velocity changes measurement (Hadziioannou et al., 2009; Lecocq et al., 2014). The SNR increases given more days are included in the stack, but the reference stack may not represent a background state if a strong velocity variation is involved (Duputel et al., 2009; Mordret et al., 2010).

The choice of the current stack size has a trade-off that the longer the time of stacking, the higher the coherence with the reference and the easier for the delay time measurement, but the lower the temporal resolution and therefore some short time variation may not be able to be detected (Lecocq et al., 2014; Yates, 2018). Different moving window size choices may over-estimate or hide the features in the reference and current stack (Liu et al., 2010). The stack size choices for reference stack and current stack are discussed in Chapters 3 and 4.

The delay time computation is carried out by the moving-window cross spectrum analysis (MWCS), which measures delay times in the frequency domain (Ratdo- 
mopurbo and Poupinet, 1995; Clarke et al., 2011; Lecocq et al., 2014). This method allows the frequency range of coherence to be defined clearly. The cross-spectrum is defined as

$$
X(\nu)=F_{r e f}(\nu) \times F_{c u r}^{*}(\nu)
$$

where $F_{\text {ref }}(\nu)$ and $F_{\text {cur }}(\nu)$ are Fourier-transformed reference and current stacks respectively, from a time window defined by the user. The asterisk denotes the complex conjugation. $X(\nu)$ is then smoothed by convolution with a Hanning window (Lecocq et al., 2014).

The cross coherence between the reference stack and current stack in the frequency domain is defined as

$$
C(\nu)=\frac{|\overline{X(\nu)}|}{\sqrt{\left|\overline{F_{\text {ref }}(\nu)}\right|^{2}\left|\overline{F_{\text {cur }}(\nu)}\right|^{2}}}
$$

where the overlines indicate the smoothing of the energy spectra for $F_{r e f}$ and $F_{c u r}$ and of the spectrum of $X$.

The unwrapped phase of the cross-spectrum is

$$
\phi_{j}=2 \pi \delta t \nu_{j}
$$

where $\delta t$ is delay time measured in user-defined moving windows using movingwindow cross spectrum analysis (Sens-Schönfelder and Wegler, 2006). $\nu_{j}$ represents the frequencies sampled in a bandwidth defined by the user. Relative time difference $\mathrm{dt} / \mathrm{t}$ is obtained by a linear regression using the measured delay time $\delta t$ (the blue dots) at the moving windows (Figure 2.6).

The final relative velocity change $d v / v$ is calculated from the relative travel time change $d t / t$ :

$$
\frac{d v}{v}=-\frac{d t}{t}
$$

To compute $\mathrm{dv} / \mathrm{v}$ by MSnoise, parameters including the time series of reference stack, length of current stacks, length of moving window, and lag time window for the time shift computation are tested and confirmed in Chapters 3 and 4. 


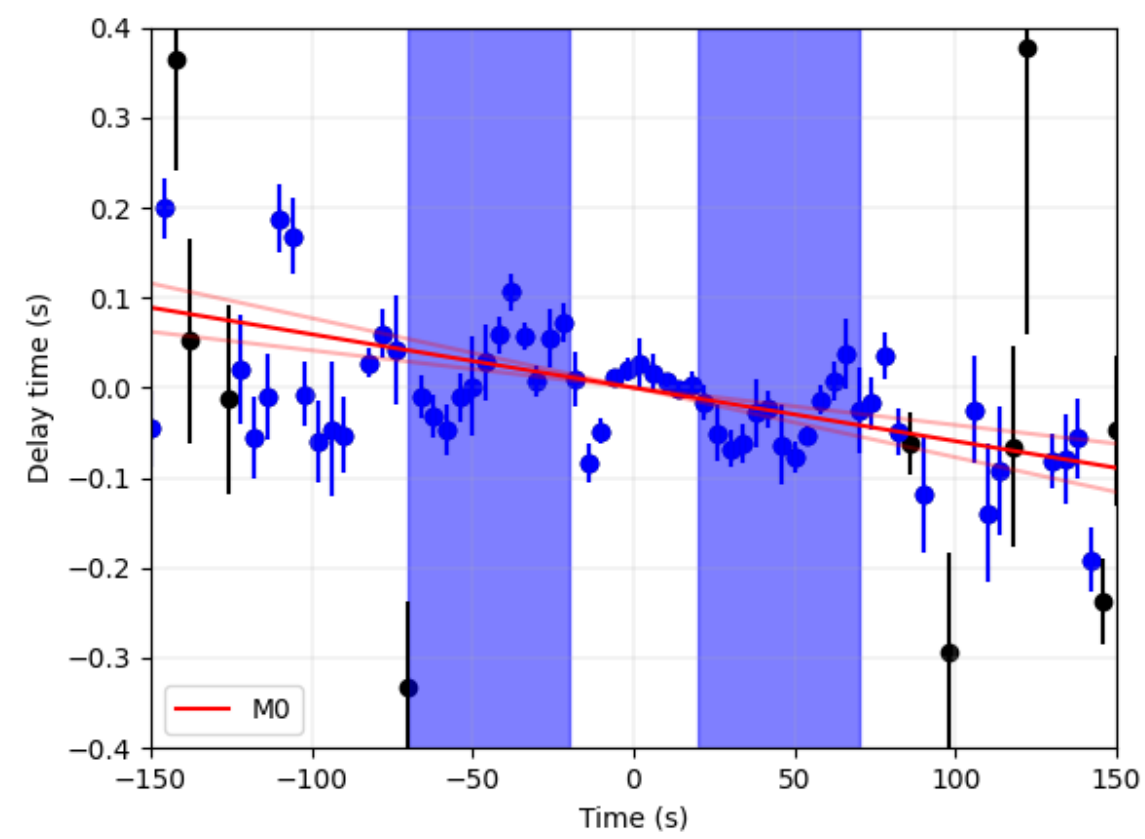

Figure 2.6: An example of dt/t measurement from LOBS8 Z-N cross-correlations at date 2014-0924. The dots with error bars are the measured delay time $\delta t$ at different moving windows. The red line (M0) shows a linear regression using the blue dots within the blue rectangles to obtain a relative time difference $\mathrm{dt} / \mathrm{t}$. The transparent red lines denote the uncertainties. The red lines are forced to cross $(0,0)$. The blue rectangles mark the coda window used for measurement. The definition of this window is discussed in later chapters. The black dots are the ones that do not meet the criteria during the measurement, e.g., $\delta t$ is larger than a threshold. The set of thresholds can be found in later chapters.

\subsection{Phase velocity measurement and shear velocity in- version}

In Chapter 5, after stacking CCFs (Section 2.4), phase velocities are measured from the stacks, and shear velocities are inverted using the phase velocities. As stated in Sections 2.2 and 2.4, the stacks of cross-correlation functions at two stations over a long time can be used as Empirical Green's functions between the two stations. In general, the most pronounced direct arrivals on the vertical-vertical components, including seismic vertical channel and pressure gauge, of the stacked CCFs, are fundamental mode Rayleigh waves propagating in the upper crust. Because the strength of ocean microseisms decays rapidly at periods $>20 \mathrm{~s}$, the contribution to the Empirical Green's functions from noise sources with frequencies lower than the spectral band of primary microseism is relatively small (Webb, 1998). In con- 
trast, higher-frequency energy of earthquake-generated surface waves tends to be attenuated and scattered more effectively during long-distance wave propagation. As such, the noise-extracted surface waves preserve better short-period dispersion information than teleseismic surface waves to constrain the shallow structure under the stations, especially the shear wave velocities to which the measured phase and group velocities are most sensitive (Stein and Wysession, 2009).

\subsubsection{Phase velocity measurement}

While group velocity represents the velocity of wave envelope, phase velocity is the velocity of wave phase (Figure 2.7).

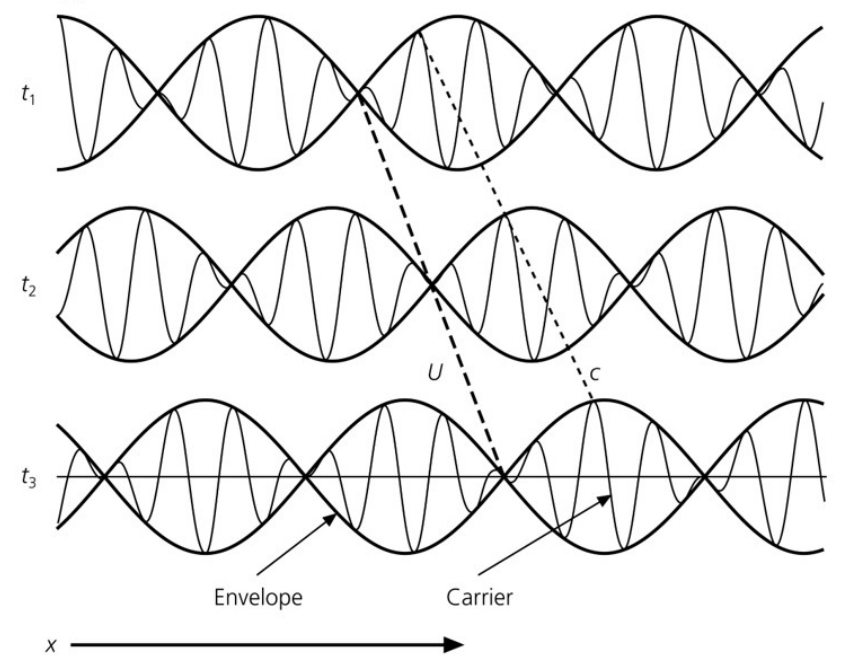

Figure 2.7: Illustration of group and phase velocities. The sinusoidal wave propagates along with time $\mathrm{t} 1, \mathrm{t} 2$, to $\mathrm{t} 3$. $\mathrm{U}$ indicates group velocity while $\mathrm{c}$ indicates phase velocity. [Figure from Stein and Wysession (2009)]

In this study, we employ frequency-time analysis (FTAN) (Levshin et al., 1992; Levshin and Ritzwoller, 2001) to measure frequency-dependent phase velocities of Rayleigh waves derived from the CCF stacks of ambient noise recorded on the seismic vertical components and differential pressure gauges between all available OBS pairs. FTAN first transfers a stack to the frequency domain, then a set of narrow-band Gaussian filters are applied,

$$
F\left(\omega-\omega_{i}\right)=\exp \left[-\alpha_{i}\left(\frac{\omega-\omega_{i}}{\omega_{i}}\right)^{2}\right], i=1, \ldots, N
$$

where $\omega_{i}$ is the central angular frequency, $\alpha_{i}$ controls the relative width of filters, $N$ is the number of filters. The filter output can be considered as a discrete image 
of the frequency-time representation of the input time series,

$$
S\left(\omega_{i}, t_{j}\right)=\int_{-\infty}^{\infty} W(\lambda) F\left(\lambda-\omega_{i}\right) \exp \left(\sqrt{-1} \lambda t_{j}\right) d \lambda, i=1, \ldots, N, j=1, \ldots, M
$$

where $W(\lambda)$ is the CCF stack in the frequency domain, $\lambda$ is wave length, $\mathrm{M}$ is the number of points at the filtered outputs. For the $i$ th filter, assuming the corresponding index in time domain is $j,\left|S\left(\omega_{i}, t_{j}\right)\right|$ is the envelope and $\arg S\left(\omega_{i}, t_{j}\right)$ is the temporal phase of the $i$ th narrow-band filter output. The FTAN code then automatically searches for the time $t_{\max }\left(\omega_{i}\right)$ corresponding to $\max \left|S\left(\omega_{i}, t_{j}\right)\right|$ for all frequencies $\omega_{i}$. Then phase velocity is estimated

$$
C(\omega)=\frac{d}{t_{\max }(\omega)-(\Phi(\omega)-\pi / 4+2 n \pi) / \omega}
$$

where $d$ is inter-station distance, $n$ is the number of cycles that a wave travels between the station pair (which is unknown), phase $\Phi$ for ambient noise crosscorrelation function (Tsai, 2009) is

$$
\Phi(\omega)=-k d+\pi / 4+\omega t_{t}
$$

where $k$ is the wavenumber, $t_{t}$ is the travel time between the station pair.

For a station pair, the phase velocity is measured at each frequency, forming a dispersion curve. Dispersion curves measured from available station pairs are then used to invert for the 1D models of shear velocities in the shallow crust.

\subsubsection{Bayesian inversion and Markov Chain Monte Carlo}

From measured dispersion curves, 1D inversion can be conducted. 1D inversion uses one measured dispersion curve, or one dispersion curve averaged from multiple dispersion curves, to invert a 1D shear velocity profile varying in depths.

The Bayesian method proposes many possible models using a Markov Chain Monte Carlo sampling algorithm. Markov Chain Monte Carlo is a sampling method in model space. It seeks the posterior probability distribution of the sample models based on the data and prior model information. By constructing a Markov chain with the desired distribution, one can obtain a sample of the desired distribution by recording states from the chain. One of the merits of the Bayesian inversion is that the data uncertainty and the number of unknown model parameters are parts of the model parameters. It provides a stable solution with no need for explicit regularization acting on the model parameters (Bodin and Sambridge, 2009). 
Given the observed data, $d^{o b s}$, the Bayesian interference aims to measure a posteriori probability distribution, $p\left(m \mid d^{\text {obs }}\right)$, e.g., the probability density of the model parameters, $m$. According to Bayes' theorem (Bayes, 1763), the posteriori probability density function relates to the priori information on the model with the observed data,

$$
p\left(m \mid d^{o b s}\right)=\frac{p\left(d^{o b s} \mid m\right) p(m)}{p\left(d^{o b s}\right)}
$$

where $p\left(d^{o b s} \mid m\right)$ is the likelihood function, which expresses the probability of having a particular model $m$ conditional on the observed data, and $p(m)$ is the priori probability density of $m$ known before measuring the data $d^{o b s}$. The inversion solution is given by a joint probability density function (PDF) in the parameter and data space, which contains the ensemble of acceptable models as allowed by data and prior information (Tarantola, 2005).

A Markov Chain Monte Carlo (Guo et al., 2016; Yang et al., 2020) is used to search for the Vs models. Phase velocity dispersion curves are forward-modeled using Computer Programs in Seismology (CPS) (Herrmann, 2013). CPS is developed by Saint Louis University, providing programs for synthetic dispersion curves for a given layered model. At each iteration of the chain, a new model conditional only on the current model $m$ is drawn from a probability distribution $q(m / \mid m)$. After $\mathrm{N}$ iterations, $\mathrm{M}$ final models are used to construct the posterior probability density function (PDF).

\subsection{D sensitivity kernel}

Sensitivity kernels provide information on how sensitive waves are to a model. A higher kernel value indicates that the waves have a better constraint on the model. The sensitivity kernels of Rayleigh wave phase velocity are calculated in Chapters 3, 4, and 5. Sensitivity kernels of Rayleigh wave group velocity and Love wave phase and group velocities are calculated in Chapters 3 and 4 .

Using Rayleigh wave phase velocity as an example (Stein and Wysession, 2009), assume there are fractional perturbations in shear wave velocity $\delta \beta / \beta$, primary wave velocity $\delta \alpha / \alpha$, and density $\delta \rho / \rho$, varying with depths $(z)$. The frequencydependent phase velocity perturbation $\delta c(f)$ caused by the model perturbation 
$(\delta \beta / \beta, \delta \alpha / \alpha, \delta \rho / \rho)$ is given by

$$
\delta c(f)=\int_{0}^{z}\left(K_{\beta}(f, z) \frac{\delta \beta}{\beta}(z)+K_{\alpha}(f, z) \frac{\delta \alpha}{\alpha}(z)+K_{\rho}(f, z) \frac{\delta \rho}{\rho}(z)\right) d z
$$

where $K_{\beta}, K_{\alpha}$, and $K_{\rho}$ are the sensitivity kernels that relate the phase velocity perturbation $\delta c(f)$ to the fractional perturbations of $\beta, \alpha$, and $\rho$, respectively. In this thesis, $\alpha$ and $\rho$ are fixed, and therefore $\delta \alpha$ and $\delta \rho$ are equal to zero.

\subsection{Data and method comparison of the two study re- gions}

Table 2.1 summarizes a comparison of the data, stations, and methods used for the studies in the southwestern Okinawa Trough and the northern Hikurangi.

Table 2.1: A comparison of data and methods used in southwestern Okinawa Trough and the Hikurangi studies.

\begin{tabular}{|l|l|l|}
\hline & $\begin{array}{l}\text { Southwestern Okinawa } \\
\text { Trough }\end{array}$ & Northern Hikurangi (NZ) \\
\hline Data/station & $\begin{array}{l}\text { Broadband + short-period (3 } \\
\text { s and 7 s) }\end{array}$ & $\begin{array}{l}\text { Broadband + short-period (1 } \\
\text { s) }\end{array}$ \\
\hline & $\begin{array}{l}34 \text { stas at various periods } \\
10 \text { stas 2014-2015; 5 stas } \\
2010-2018\end{array}$ & $\begin{array}{l}\text { Single sta cross component } \\
\text { correlation }\end{array}$ \\
\hline Techniques & Cross sta correlation & $\mathrm{dv} / \mathrm{v}$ \\
\hline & Phase velocity measurement \\
\hline & Vs inversion & $\mathrm{dv}$ \\
\hline
\end{tabular}




\section{Chapter 3}

\section{Temporal velocity variations in the northern Hikurangi margin and the relation to slow slip}

Weiwei Wang, Martha K. Savage, Alec Yates, Hubert J. Zal, Spahr Webb, Carolyn Boulton, Emily Warren-Smith, Megan Kortink, Tim Stern, Bill Fry, Kimihiro Mochizuki, Laura Wallace, submitted to Earth and Planetary Science Letters on 14th March 2021

\subsection{Abstract}

Slow slip events (SSE) have been studied in increasing detail over the last 20 years, improving our understanding of subduction zone processes. Although the relationship between SSEs and the physical properties of their surrounding materials is still not well-understood, the northern Hikurangi margin in New Zealand is the site of relatively shallow ( $<10 \mathrm{~km}$ deep), frequent SSEs, providing excellent opportunities for near-field investigations. From September to October 2014, an SSE occurred with more than $250 \mathrm{~mm}$ slip, and was recorded successfully by the Hikurangi Ocean Bottom Investigation of Tremor and Slow Slip (HOBITSS II) deployment. This study applies coda wave interferometry to ambient noise data acquired by nine HOBITSS II ocean bottom seismometers (OBS) to study the seismic velocity variations in the upper crust of the overriding plate related to the SSE. Single station cross-component correlations are computed within a period band 
that focuses on the upper plate in our study region. The average velocity variations display a decrease on the order of $0.05 \%$ during the SSE, followed by an increase of similar magnitude afterwards. We suggest that two possibilities exist to explain this. The first possibility, which has been suggested by other seismological observations, is that the SSE causes a low-permeability seal on the plate boundary to break. The break allows fluid to migrate into the upper plate, causing a seismic velocity decrease during the SSE because of increased pore fluid volume in the upper plate. Under this model, after the SSE, the fluids in the upper plate diffuse gradually and the velocity increases again. The second possibility is the velocity changes are related to changes in crustal strain during the slow slip cycle, whereby elastic strain accumulates prior to the SSE causing contraction and reduction of porosity and therefore increase of velocity above the SSE source (the seismic velocity increases between SSEs). During the SSE the upper plate goes into extension as the elastic strain is released, which results in dilation and a porosity increase (seismic velocity reduction). After the SSE, stress and strain accumulate again, causing a porosity decrease and a velocity increase.

\subsection{Introduction}

Slow slip events (SSE), involving fault slip over days to years, have been a topical field of tectonics and fault mechanics research over recent years as they extend our understanding of subduction zone deformation. The occurrence of SSEs, and how this relates to the physical properties of surrounding materials remains an outstanding question, although increasing numbers of geophysical observations from subduction zones globally are helping to constrain this relationship. Rivet et al. (2011) observed a seismic velocity decrease during an SSE in Mexico in 2006 and suggested the velocity decrease was related to the strain rate in the overlying crust. Frank et al. (2015) observed variations of low frequency earthquake occurrence rates during the same SSE in Mexico, suggesting that fluids play an active role in SSE source regions. Nakajima and Uchida (2018) found variations in seismicity rates and seismic attenuation with the cyclic occurrence of SSEs in Kanto, Japan, which were interpreted to represent intensive drainage during slow slip events. Gosselin et al. (2020) observed velocity changes after episodic tremors and slow slip events in Cascadia, which they interpreted to reflect fluctuations in pore fluid pressure. Recent investigations of SSEs in the northern Hikurangi 
showed temporal stress ratio variations (Warren-Smith et al., 2019), Vp/Vs ratio variations and shear wave splitting delay time changes (Zal et al., 2020) during the SSEs, suggesting build-up of pore fluid pressure on the plate boundary prior to the SSEs, and release during SSEs. Typically, these studies focus on specific parts of the subduction system (e.g. upper or lower plates), or are sensitive to different rates of changes, so additional techniques which help to constrain the timescales and magnitudes of stress and fluid pressure variability are required to complement previous methodologies. The Hikurangi margin, which has recently hosted several amphibious geophysical deployments focused on recording slow slip processes, provides an outstanding natural laboratory to test these methods in tandem.

The Pacific Plate subducts beneath the Australian Plate along the Hikurangi margin with a convergence rate of $50 \mathrm{~mm} / \mathrm{yr}$ at the northern Hikurangi subduction zone (Wallace et al., 2004). The plate interface is located at 10-15 km depth beneath the east coast of the North Island, which is much shallower than many other subduction zones in the world, where the equivalent shallow plate interfaces are typically located $>50-100 \mathrm{~km}$ offshore. This has enabled identification of shallow (<10 km depth) SSEs using land-based geophysical networks (e.g., Douglas et al., 2005; Wallace and Beavan, 2010). The Hikurangi margin can be divided into three segments: northern, central and southern. Shallow SSEs occur in the northern and central segments, while deep SSEs (>25-30 km depth) are observed in the southern segment (Wallace et al., 2009; Wallace and Beavan, 2010). This study focuses on shallow SSEs at the northern Hikurangi margin.

The northern Hikurangi is the site of some of the world's shallowest, well-documented SSEs. SSEs have been observed in the Hikurangi subduction zone since continuous GNSS sites started to be installed in 2002 (e.g., Douglas et al., 2005; Wallace and Beavan, 2010). SSEs offshore Gisborne occur approximately every 1-2 years, and are detected by continuously operating GNSS sites near the east coast of the North Island. To provide improved resolution of SSE processes on the offshore plate boundary, the HOBITSS II project deployed 24 absolute pressure gauges and 15 ocean bottom seismometers (OBSs) from mid-2014 to mid-2015. Four SSEs occurred during this deployment (SSE1-SSE4); the locations of these SSEs are shown in Figures 3.1 and A.2. Warren-Smith et al. (2019) calculated the timing of these SSEs from time-dependent geodetic inversions. SSE1 occurred to the south of the 
deployment region from 1st September to 17th November 2014. SSE2 occurred from 24th September to 2nd November 2014 directly beneath the HOBITSS II network. SSE3 occurred from 18th December 2014 to 12th January 2015. The main slip of SSE3 occurred mostly to the south of the HOBITSS II deployment but also within part of the HOBITSS II deployment region. SSE4 occurred from 7th February to 1st March 2015 outside of the deployment region. A primary focus of this study is SSE2, which had the largest slip. The main period of slip occurred over 2 to 3 weeks, equivalent to a moment magnitude of Mw 6.8, with the main pulse of slip from late September to mid-October, with a tailing off period from mid to late October (Wallace et al., 2016; Yohler et al., 2019). The largest slip (more than 200 $\mathrm{mm}$ ) occurred at a depth of 4-7 km beneath the central portion of the HOBITSS II network (Wallace et al., 2016).

In subduction zones, increased pore fluid pressure due to mineral dehydration and compaction likely influences the mechanism of earthquakes and SSEs (Saffer and Tobin, 2011; Chaves and Schwartz, 2016). Warren-Smith et al. (2019) calculated earthquake focal mechanisms of local microseismicity using the data recorded by the HOBITSS II deployment to determine the stress ratio variations within the subducting oceanic crust during the SSE cycle. The stress ratio $R_{r e t r}=\left(\sigma_{1}-\sigma_{2}\right) /\left(\sigma_{1}-\right.$ $\left.\sigma_{3}\right)$ is retrieved from focal mechanism inversion, where $\sigma_{1}, \sigma_{2}$ and $\sigma_{3}$ are the maximum, intermediate, and minimum principal stresses, respectively. Because the Pacific plate is subducting at a shallow angle, $\sigma_{1}$ is nearly perpendicular to the interface and $\sigma_{3}$ is oriented down-dip and aligned with tensional slab pull. Pore fluid pressure has a negative correlation to the retrieved stress ratio (Martínez-Garzón et al., 2016). Observations by Warren-Smith et al. (2019) showed that the stress ratio in the lower plate decreased before SSE2 and increased during SSE2, suggesting pore fluid pressure increased in the lead-up to SSE2, and then decreased during SSE2, and similar patterns occurred during the other three SSEs observed during the HOBITSS II. This was interpreted as increased fluid pressurization below a low-permeability barrier prior to slow slip, and subsequent fluid migration arising from increased strain induced permeability during slow interface rupture. Zal et al. (2020) used local earthquake events with seismic ray paths largely traveling through the upper plate to compute shear wave splitting and $\mathrm{Vp} / \mathrm{Vs}$ ratios at the HOBITSS II OBS sites. They found the $\mathrm{Vp} / \mathrm{Vs}$ ratios increased and shear wave splitting delay times decreased during SSE2, implying the amount of fluid in the 
upper plate increased during SSE2. Their study suggested fluid accumulation and release before and during SSE2 as SSE2 broke the permeability seal on the plate boundary and permitted the required fluid interconnection, which was broadly consistent with the observations and model of Warren-Smith et al. (2019).

Seismic velocity variation calculations using ambient noise coda wave interferometry have been widely used in the last fifteen years to monitor the changes of subsurface material properties, including those caused by large earthquakes (e.g., Brenguier et al., 2008; Minato et al., 2012; Froment et al., 2013). Rivet et al. (2011) used this method to study a SSE in Mexico in 2006 with a size equivalent to an M7.5 earthquake. They observed a velocity decrease in the upper and middle crust during the SSE and suggested it was related to the strain rate rather than the strain, suggesting the overlying crustal deformation showed nonlinear elastic behavior during the SSE. For the same SSE, Frank et al. (2015) suggested there was a pore pressure fluctuation that migrated updip along the subduction interface according to the variations of the low frequency earthquake occurrence rates during that event. They suggested that fluids play an active role in SSE source regions. Velocities of coda waves retrieved from ambient noise cross-correlations are sensitive to fluids. This paper uses seismic ambient noise data recorded by the HOBITSS II instruments to resolve the velocity variations in the upper crust of the overriding plate related to SSEs and to examine potential implications for changes in physical properties of the crust through the SSE cycle. Here we propose the hypotheses that occurrence of SSEs is accompanied with the changes of strain or/and fluid volume which results in seismic velocity changes. For example, if SSE occurrence causes increased fluids or decreased strain, the seismic velocity is expected to decrease.

\subsection{Data}

From May 2014 to June 2015, the HOBITSS II project deployed ten broadband OBSs from Lamont Doherty Earth Observatory (LOBS) with a velocity response flat down to $100 \mathrm{~s}$, and five short period OBSs from Earthquake Research Institute, Japan (EOBS) with a natural period of $1 \mathrm{~s}$, offshore from Gisborne (Figure 3.1). Three stations, LOBS2, LOBS3, and LOBS4 were deployed on the subducting Pacific Plate as reference sites, while the remaining stations were deployed on the overlying Australian plate to record signals associated with the underlying slow 


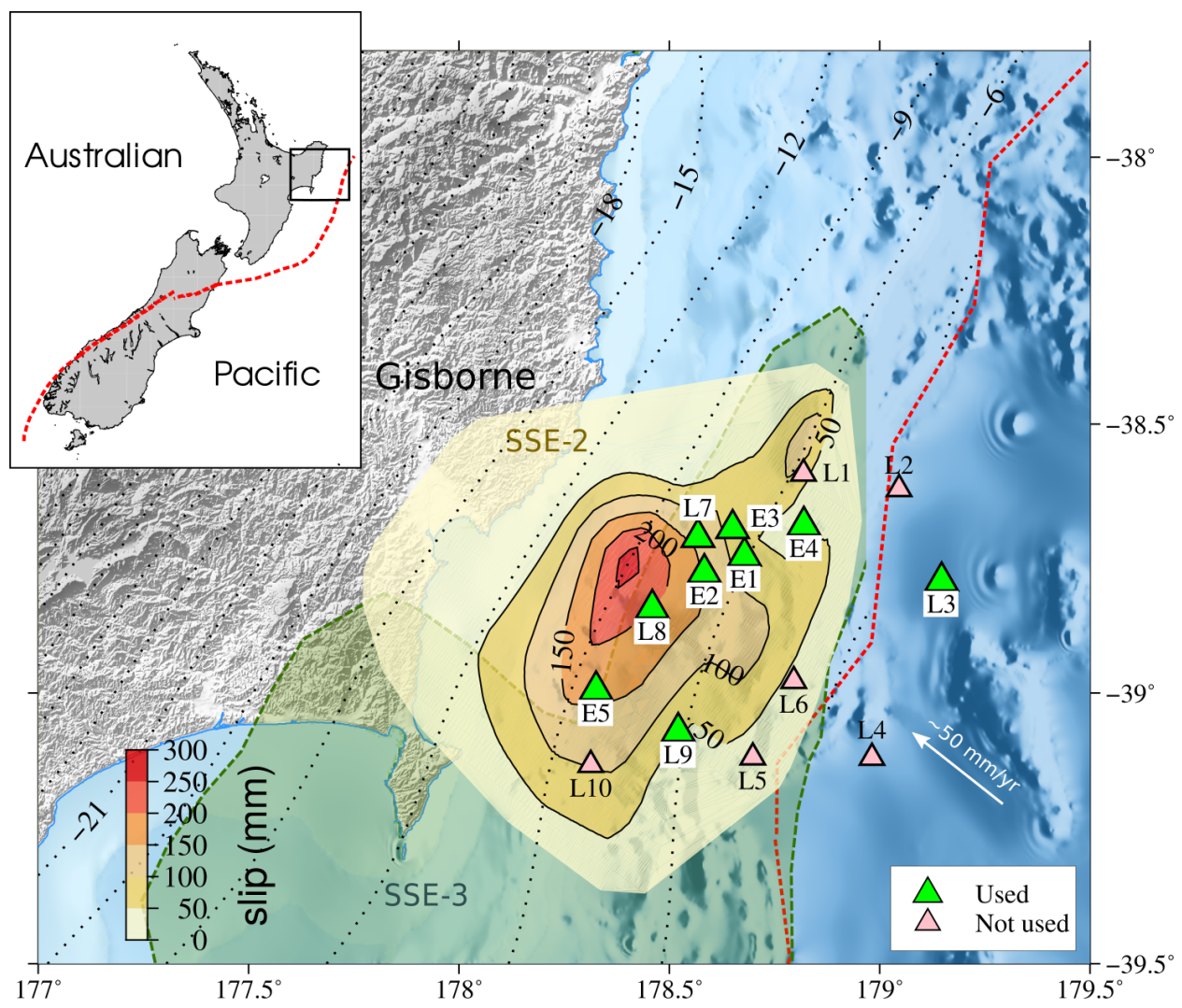

Figure 3.1: Locations of ocean bottom seismometers (OBSs; TAN1405 Science Party, 2014) with the contours showing the slip from SSE2 occurring from September to October 2014 (Wallace et al., 2016). The green shade shows the location of SSE3, which is shown in full in the map in supplementary Figure A.2. Station names are shortened from LOBS to L and EOBS to E. The green triangles denote the stations used in this study and the pink stations are not used. The dashed red line shows the subduction trench (Coffin et al., 1998) and black dotted contours show plate interface depths in $\mathrm{km}$ (Williams et al., 2013).

slip region.

The continuous data recorded from May 2014 to June 2015 by three component seismometers of nine OBSs (green triangles in Figure 3.1) are used to compute single station cross component correlation functions. Stations LOBS1, LOBS2, LOBS4, LOBS5, and LOBS10 did not acquire sufficient data and are not used in our analysis. The orientations of LOBS6 horizontal components were not able to be determined (Zal et al., 2020) and therefore these data are also not used in this study. We use the coda waves of cross-correlations to monitor velocity variations. Cross station correlations are not included in the main results because of instrument timing issues, which do not influence single station correlations.

During processing, horizontal station components are rotated to be parallel and 
perpendicular to the coastline according to the orientations determined by $P$ waves and Rayleigh waves of teleseismic events (Zal et al., 2020), as we expect the structure to follow the coastline. Coda waves are derived from single station verticalparallel cross correlations, vertical-perpendicular cross-correlations, and parallelperpendicular cross-correlations.

\subsection{Methods}

Velocity variations can be determined using coda waves retrieved from single station cross component correlations, which represent scattered waves traveling out from the station and back at later time. The further the waves travel, the later they appear in the cross-correlation function. Seismic noise data are processed through to velocity changes using a Python package MSNoise 1.5 (Lecocq et al., 2014). Parameters in MSNoise are tested, evaluated, and determined, with the aim of maximizing the lag-time dependent signal-to-noise ratio (SNR; Larose et al., 2007; Clarke et al., 2011) of cross-correlation functions (e.g., Yates, 2018). The coherence and correlation coefficient between current stacks and reference stacks are calculated to evaluate data quality (Supporting Information S1).

Each station is processed independently. Three-component daily noise records of each station are down sampled to $20 \mathrm{~Hz}$, and band pass filtered from 2.5 to 20 $\mathrm{s}$. Time domain normalization is applied through clipping data at three times the Root-Mean-Square, followed by spectral whitening. Daily series are then cut to $14400 \mathrm{~s}$ segments for LOBSs and $1800 \mathrm{~s}$ segments for EOBs, with $70 \%$ overlap in window length, to correlate, because these segment lengths of cross-correlations have higher SNR than other lengths (Figure A.5). The cross-correlations are then linearly stacked to determine daily cross-correlations. We evaluate the quality of each daily stack by computing the correlation coefficient between these and the stack of all days for a single station. This allows us to exclude daily crosscorrelations if they fall below a threshold correlation coefficient value (e.g., Figure A.6). The value varies from station to station ( 0.1 to 0.65$)$ in order to guarantee a sufficient number of daily cross-correlations for velocity change computation, while discarding the lowest quality cross-correlations for each station. The correlation coefficients of some stations are generally low because OBSs have higher noise levels than land stations. The daily cross-correlations after selection are re- 
stacked as a reference. Figure A.7 shows the final available cross-correlations of all the used stations and Figure A.8 shows the reference stacks.

Velocity changes are determined by measuring the delay time between smaller 'current' stacks and a reference stack over different lag times. A velocity change is then computed by fitting a slope to delay times, where $-\mathrm{dt} / \mathrm{t}=\mathrm{dv} / \mathrm{v}$, under the assumption of a homogeneous velocity change (Clarke et al., 2011; Lecocq et al., 2014). A positive value in $\mathrm{dv} / \mathrm{v}$ represents a velocity increase while a negative value in $\mathrm{dv} / \mathrm{v}$ represents a velocity decrease. A zero value in $\mathrm{dv} / \mathrm{v}$ means there is no velocity change relative to the background velocity. A reference stack would ideally represent the background state of the study region. Multiple references stacks are tested, including a linear stack of all available days and stacks using only daily stacks prior to SSE1 and SSE2 (Figure A.10). We observe similar velocity variations regardless of the choice of reference stack, so opt to use the reference stack of all available days going forward. Current stacks are chosen to be linear stacks of 20 daily cross-correlation functions (Figure A.6). This stacking improves the SNR of the coda signal by suppressing random noise. The current stack for a given date then represents the stack of that day and 19 days before it. If there is a velocity change on the given date, the change would start to be seen at that time. Figure 3.2 shows an example of the current stacks and the reference stack from one station.

Coda waves are used to measure the delay time because they travel longer distances, and the delay time between the current stacks and the reference stack is easier to identify on the coda than the direct arrivals. The coda wave window is determined to be -70 to -20 seconds and 20 to 70 seconds on the lag times of the stacks, according to the SNR (Supporting Information S1). We use the movingwindow cross-spectral analysis method (Clarke et al., 2011; Poupinet et al., 1984) to measure the time differences between the current stacks and the reference stack. The measurement is carried out in the frequency domain in a series of 20-second windows with a 4-second moving step within the coda wave window. A current stack must be similar to the reference stack to ensure its quality. Coherence between a current stack and the reference stack is computed and a threshold of coherence is set to 0.89 to exclude noisy current stacks (Figure A.9). The data in a frequency band of 2.5-14 s are used when applying the moving-window crossspectral analysis method. In our study region, surface waves with periods up to 14 


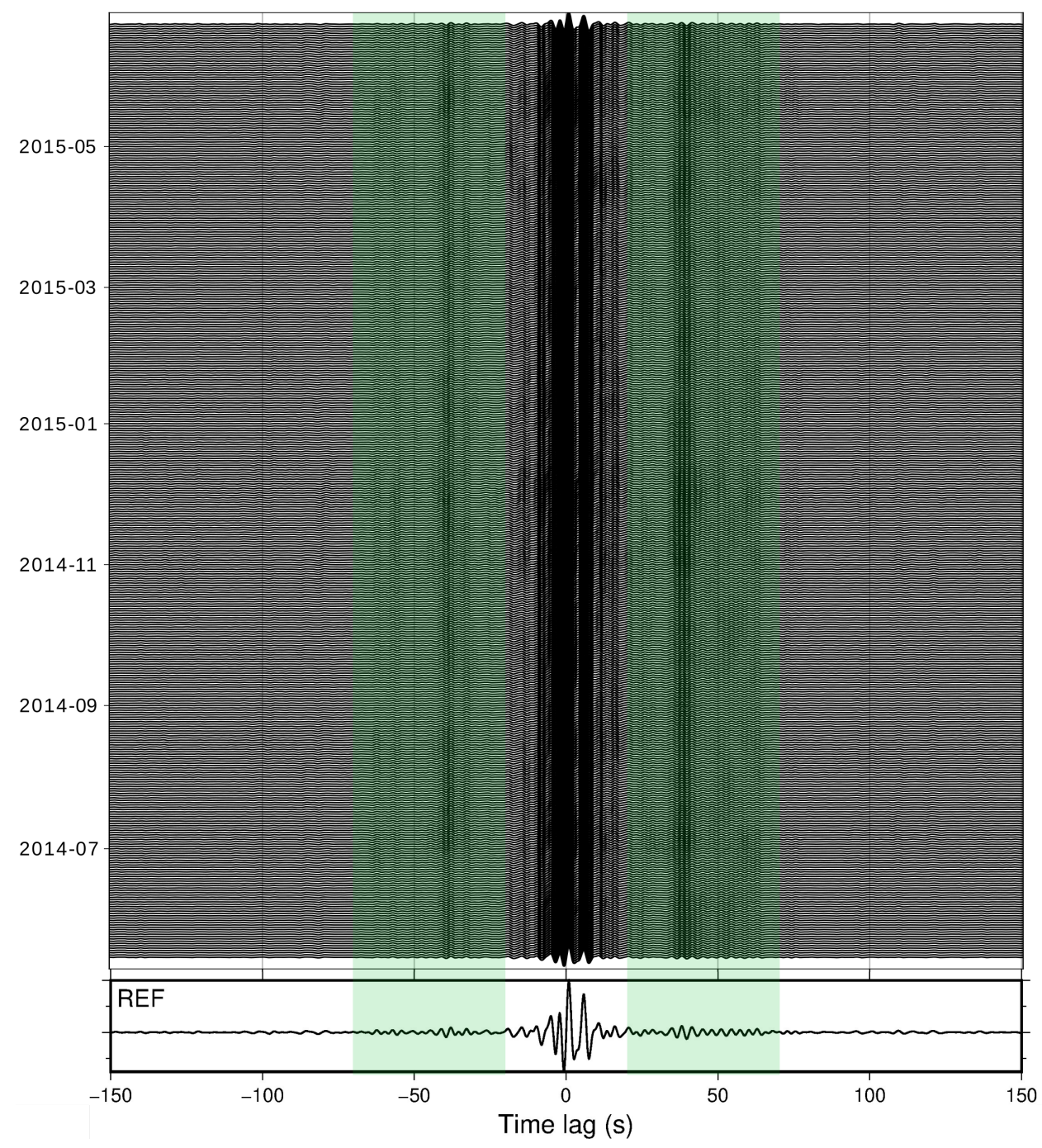

Figure 3.2: 20-day current stacks over time from EOBS1 vertical-perpendicular component correlations and corresponding reference stack. The green shades mark the window (-70 s to $-20 \mathrm{~s}$ and 20 $\mathrm{s}$ to $70 \mathrm{~s})$ to compute velocity variations.

$\mathrm{s}$ are most sensitive to properties of the upper plate (Figures 3.3 and 3.4). The first overtone mode is considered because observations of seafloor ambient noise show a transition from most of the noise energy in the fundamental mode at long periods to the first overtone mode at shorter period (<6 s; Harmon et al., 2007; Russell et al., 2019). Deeper depths (the subducting plate) require longer period observations. We do not concentrate on longer-period observations relevant to the lower plate because scattered waves used for computing velocity variations are mainly short period (Aki, 1981). Moreover, the EOBSs are short-period instruments with a natural period of $1 \mathrm{~s}$.

Relative velocity variation $\mathrm{dv} / \mathrm{v}$ is then determined by $\mathrm{dv} / \mathrm{v}=-\mathrm{dt} / \mathrm{t}$, and corre- 


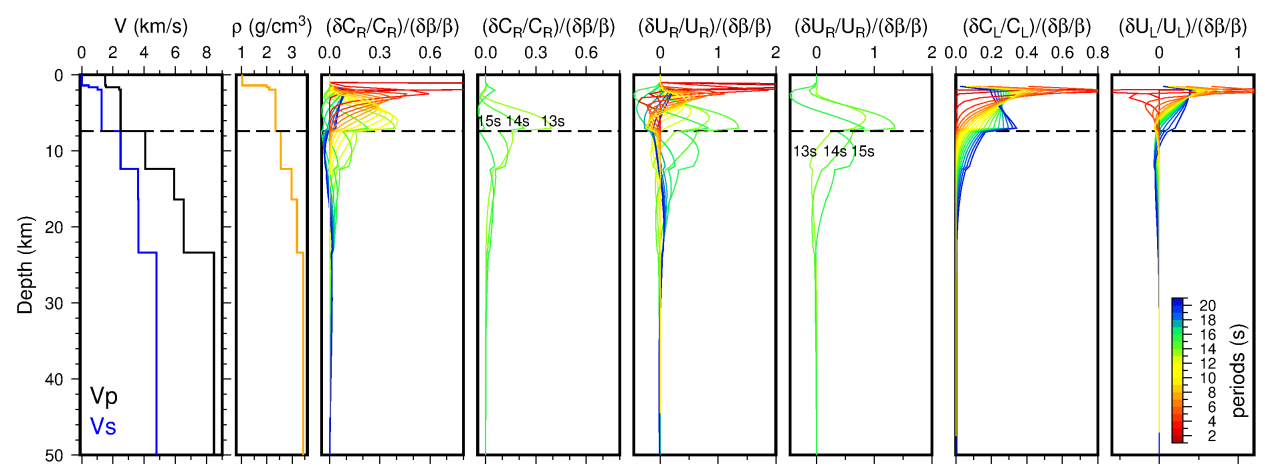

Figure 3.3: Velocity and density models in the study region and corresponding sensitivity kernels of fundamental mode Rayleigh wave phase velocities $\left(C_{R}\right)$ and group velocities $\left(U_{R}\right)$, fundamental mode Love wave phase velocities $\left(C_{L}\right)$ and group velocities $\left(U_{L}\right)$, with respect to shear wave velocities. The sensitivity kernels are color-coded according to corresponding periods. Velocity models are from Yarce et al. (2019), combined with the information of Vp and Vs from ODP U1519. The density model is from New Zealand Wide model 2.2 (Eberhart-Phillips et al., 2020) with the top layers from ODP U1519. The plate boundary is at a depth of about $7.4 \mathrm{~km}$ in the center of our study region, shown by the horizontal dashed line. The sensitivity kernels show the waves with periods shorter than $14 \mathrm{~s}$ are sensitive to the upper plate, while the waves with periods longer than $15 \mathrm{~s}$ are sensitive to the lower plate. In the frequency domain, we use the data up to $14 \mathrm{~s}$ to compute $\mathrm{dv} / \mathrm{v}$ to focus on the upper plate.

sponding errors are estimated (Lecocq et al., 2014). For a single station, $\mathrm{dv} / \mathrm{v}$ from the three cross-components is computed and averaged, using the median and mean values. Component-averaged $\mathrm{dv} / \mathrm{v}$ on single stations are then averaged from LOBS7, LOBS8, LOBS9, and all EOBSs. The errors of the average $\mathrm{dv} / \mathrm{v}$ are calculated by a bootstrap method that calculates the confidence intervals for a given sample of a population and a statistic with 200 times iteration. LOBS3 is excluded because it is located on the subducting plate where the SSE2 slip does not extend beneath. The $\mathrm{dv} / \mathrm{v}$ result from LOBS3 is also less reliable because LOBS3 recorded a shorter dataset compared to the other stations (Figure A.7).

\subsection{Results}

There were four main SSEs (Warren-Smith et al., 2019) during the deployment, from which SSE2 had the most significant displacements, in some locations over $250 \mathrm{~mm}$ (Figure 3.1; Wallace et al., 2016). Many single station results show a velocity decrease during SSE2 and an increase after it (Figure 3.5). There are also some significant changes in velocity outside the time of SSE2 in the single station results. 


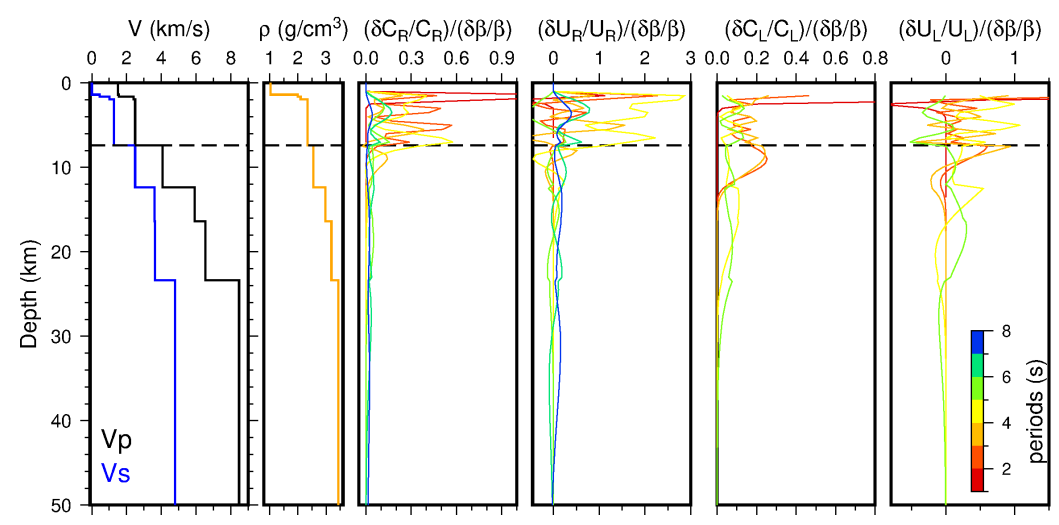

Figure 3.4: Similar to Figure 3.3 but for the first overtone mode. Differs from the fundamental mode at 2.5-6 s which is only sensitive to shallow structure (down to $4 \mathrm{~km}$ depth), the first overtone mode is also sensitive to shear velocity changes at deeper depths.

However, they are suppressed when we average the single station variations, suggesting they are more likely to be random noise.

From the average result, there is a $0.06 \% \pm 0.03 \%$ velocity decrease during SSE2 over one month and a $0.07 \% \pm 0.04 \%$ velocity increase after SSE2 over one and a half months. Subsequently, the velocity decreases $0.06 \% \pm 0.03 \%$ over 20 days, which might be caused by SSE3, followed by a small increase of $0.03 \% \pm 0.03 \%$ over 10 days returning to original values. Note that because the $\mathrm{dv} / \mathrm{v}$ value on a given date is computed from the 20 days before it, the velocity variations have some ambiguity in timing of up to 20 days. Although the error bars on the average result are large, the velocity variations during and after SSE2 are larger than other variations, and we show below that data processed by other techniques are wellcorrelated with these results.

\subsection{Discussion}

\subsubsection{Relation to SSEs}

The data in a frequency band of 2.5-14 s are used to compute dv/v. From the sensitivity kernels (Figures 3.3 and 3.4), our observations (2.5-14 s) are most sensitive down to $7.4 \mathrm{~km}$ depth, which is in the upper plate. The physical mechanism of single station cross component correlations is not yet well understood and the cross-correlations may contain other types of waves such as body waves, but we assume they are mainly surface waves following previous studies (e.g., Hobiger 

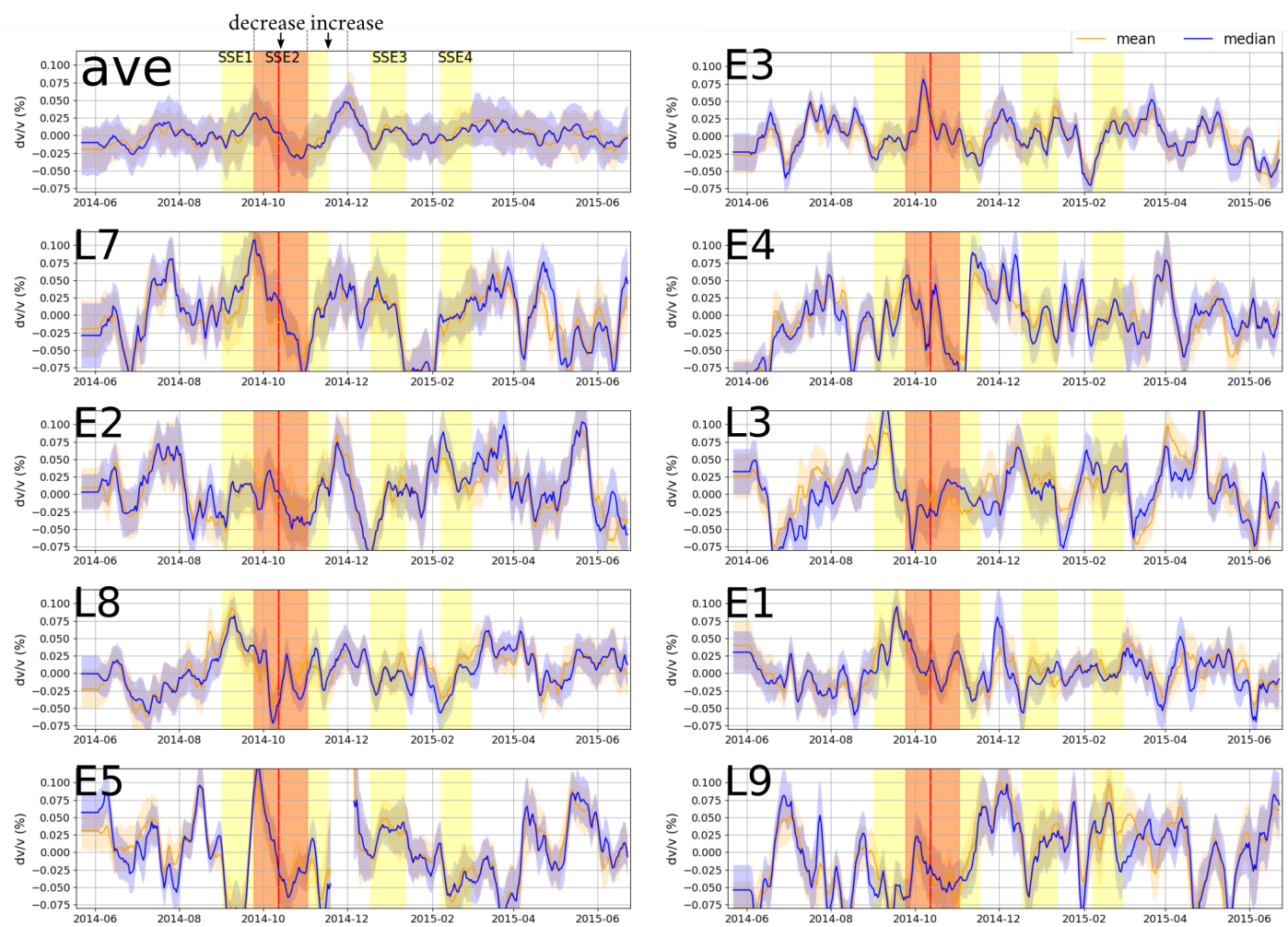

Figure 3.5: Single station time-dependent velocity variations and their average. Mean (orange) and median (blue) values of velocity variations are shown with their uncertainties for the single-station results and the average result. L3 is not included in the average because it is not in the slip region, and it lost some data (Figure A.7). The dv/v values are computed using a 20-day window and further smoothed by a 5-day window. The gap in E5 after SSE2 is because of the lack of data. Four main SSEs during the deployment are marked. The biggest event, SSE2, is marked by orange and the other three are marked as yellow. The red vertical lines mark the time when SSE2 finishes its main slip.

et al., 2014). Velocity variations with a band pass of 1 to $2.5 \mathrm{~s}$ do not show obvious temporal variations (Figure A.11), suggesting the changes are not in the very shallow portion of the crust (Figure 3.3). The temporal velocity variations at $2.5-6 \mathrm{~s}$ are similar to those at 2.5-14 s (Figure A.12), suggesting that the dominant frequencies of the velocity changes might be $2.5-6 \mathrm{~s}$. The fundamental mode at $2.5-6 \mathrm{~s}$ is only sensitive to shallow depths above $4 \mathrm{~km}$ (Figure 3.3), while the first overtone at 2.5-6 s is also sensitive to deeper depths (Figure 3.4) in the upper plate. The velocity variations at 6-14 s also show a velocity decrease during SSE2 and an increase after it (Figure A.13), indicating the data still sample the deeper region. The limitation of our data is that they do not have high resolution at depths; the results only reflect an average of the upper plate. Determining the horizontal sensitivity for single-station $\mathrm{dv} / \mathrm{v}$ measurements is not as straightforward. For a conservative 
estimate, we consider the maximum distance a surface wave could travel from the network using a velocity of $1.3 \mathrm{~km} / \mathrm{s}$ (Yarce et al., 2019) and the maximum lag time of our coda window (70s). This equates to a radius of $45 \mathrm{~km}$ around the network, approximately the distance from the network to the coast. Note, however, it could be expected that this radius is not sampled evenly, with $\mathrm{dv} / \mathrm{v}$ measurements more sensitive to changes within the network. Such local sensitivity of single-station measurements has been observed elsewhere (e.g., De Plaen et al., 2019).

Seismic velocity changes, especially for shear waves, mainly depend on fluid changes. Fluids influence seismic velocities in many different ways (Berryman, 2007). For instance, near surface water table levels in very shallow regions (hundreds of meters), bound water in minerals (mainly in the mantle and lower crust), fluid pressure and free water. In our case, the velocity variations are more likely to be linked with free water. Fluid volume increase leads to a shear velocity decrease (Grêt et al., 2006). According to the decrease in $\mathrm{dv} / \mathrm{v}$ in the station average (Figure 3.5 top left), we suggest there might be a fluid migration event or a porosity increase over approximately one month from late-September to the start of November 2014. The timing fits well with previous studies suggesting that SSE2 occurs from 24th September 2014, with its main slip taking place until mid-October, with slip tapering off from mid to late October (Wallace et al., 2016; Yohler et al., 2019). After SSE2, the seismic velocity increases gradually until around 1st December 2014, taking about one and a half months.

There is a small increase in the station-averaged velocity at the end of October 2014 during SSE2 from the 10-day moving window dv/v (Figure A.12), which is smoothed out by the 20-day moving window dv/v results. Some single station 20-day moving window dv/v results (LOBS8, EOBS1, EOBS3, and EOBS4) also show this velocity increase (Figure 3.5). Wallace et al. (2016) suggested the main slip of SSE2 happened before 12th October 2014, while the slip after that until 2nd November 2014 (Warren-Smith et al., 2019) was lower. The velocity increase at the end of October occurred during a tapering-off of slip in SSE2, after the main pulse of slip. Because only some of the stations detect this velocity change, we cannot definitively distinguish whether this is just noise, or if it is a real velocity change during the last, lower slip rate phase of SSE2.

Following the velocity increase which ends in early December 2014, the seismic 
velocity appears to undergo another decrease, which is a couple of weeks prior to the observation of SSE3 on land-based GNSS stations (Warren-Smith et al., 2019). It is plausible that this velocity decrease could be related to SSE3, if the SSE began in the offshore region earlier than it is seen at the onshore GNSS stations, similar to the progression during SSE2 (Wallace et al. (2016) suggested SSE2 may start earlier offshore). However, given that we had limited seafloor geodetic instrument coverage above SSE3 to constrain the timing of SSE3 (which is located just to the south of the HOBITSS II network), it is difficult to say if these velocity changes are related to SSE3. Although the patterns of velocity increase and decrease in this period are similar to those observed during SSE2 - there is a period of velocity decrease followed by a period of velocity increase. The timing suggested by the velocity decrease is from the beginning of December, lasting approximately 20 days. After that, velocity recovery occurs over approximately 10 days, possibly reflecting fluid diffusion and/or changes in porosity.

SSE1 and SSE4 are smaller SSEs, and did not occur under the deployment region (Warren-Smith et al., 2019) and therefore are not expected to influence observed changes in seismic velocity beneath the region to the same extent as for the larger events within the network footprint.

We suggest two hypothesis based on our observation. 1) Fluid migration related to fault-valve behavior: the velocity decrease during SSE2 is caused by more fluids migrating into the upper plate as SSE2 breaks a low-permeability seal on the plate boundary. 2) Crustal strain changes through the SSE cycle: the velocity decrease during SSE2 is caused by increased porosity because SSE2 relieves the elastic strain, which results in dilation. A combination of both processes is also possible. 


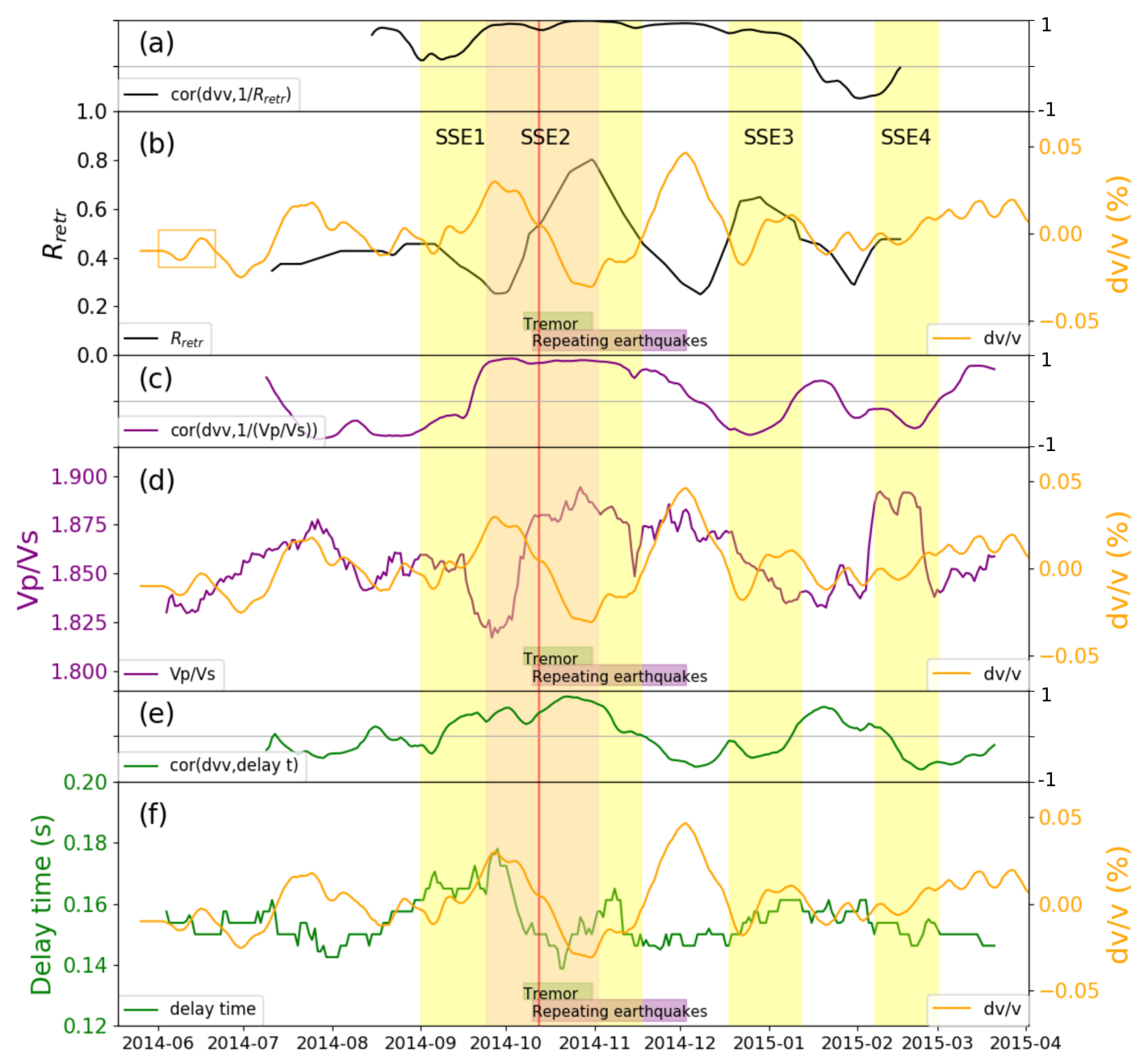

Figure 3.6: The median value of 20-day moving window station-average velocity variations compared to other studies. Four SSEs are marked and the vertical red line marks the time when SSE2 finished its main slip (Wallace et al., 2016; Yohler et al., 2019). (b), (d), (f) are to compare with other studies and (a), (c), (e) are the corresponding correlations. (a) Correlation of velocity variations and inverted stress ratio variations, computed using a 35-day moving window and a step of 1 day. The grey horizontal line marks value 0 of correlation. The top and bottom are 1 and -1 respectively. (b) Velocity variations compared to retrieved stress ratio variations Rretr. The black curve shows stress ratio changes (Warren-Smith et al., 2019), which focus on the lower plate. The orange curve shows the velocity variations which focus on the upper plate. The yellow rectangle shows a 20-day window length. The time duration of tremor (Todd et al., 2018) and repeating earthquakes (Shaddox and Schwartz, 2019) is denoted by green shade and purple shade respectively. (c) Correlation of velocity variations and inverted $\mathrm{Vp} / \mathrm{Vs}$ variations [from Zal et al. (2020)]. (d) Velocity variations compared with $\mathrm{Vp} / \mathrm{Vs}$ variations. (e) Correlation of velocity variations and delay time variations [from Zal et al. (2020)]. (f) Velocity variations compared with delay time variations. 


\subsubsection{Fluid migration related to fault-valve behavior}

The scattered waves that we use to compute velocity variations are mainly surface waves. These waves are sensitive to fluid volume, and an increase in fluid volume corresponds with a shear velocity decrease, and vice versa (Grêt et al., 2006). The velocity decrease is consistent with a fluid volume increase in the upper plate during SSE2. After SSE2, the velocity increase suggests the fluids in the upper plate diffuse over time.

Both studies of Warren-Smith et al. (2019) and Zal et al. (2020) support a 'faultvalve' hypothesis in this region of the Hikurangi margin, wherein a temporary low-permeability seal on the plate boundary maintains near lithostatic fluid pressure in the lower plate, which accumulates over time, reaching a peak prior to slow slip, which might trigger slip initiation. Following this, the occurrence of slow slip ruptures the seal and fluids migrate from the interface to the upper plate.

Here we compare the velocity variations in the upper plate with the stress ratio variations in the lower plate (Figure 3.6b; Warren-Smith et al., 2019), Vp/Vs and delay time variations in the upper plate (Figure 3.6d and f; Zal et al., 2020). The temporal stress ratio variations and velocity variations might have a time shift (Figure 3.6) because the stress ratio variations have horizontal uncertainties varying from \pm 2 to \pm 13 days (Warren-Smith et al., 2019). Zal et al. (2020) used a 20-day moving window for $\mathrm{Vp} / \mathrm{Vs}$ and delay time computations and represented the values on the middle day of the 20-day window, while our velocity variations computed on a 20-day window represent the values on the last day of the 20-day window. To better compare the results, we shift the variations of Zal et al. (2020) to the right by 10 days. The $\mathrm{dv} / \mathrm{v}$ variations around SSE2 are negatively correlated with the stress ratio (Figure 3.6b) and the $\mathrm{Vp} / \mathrm{Vs}$ variations (Figure $3.6 \mathrm{~d}$ ), and are in good positive correlation with delay time variations (Figure 3.6f). The stress ratio increases while the velocity decreases during SSE2. Since the stress ratio has a negative correlation with pore fluid pressure, this suggests a fluid pressure decrease in the top of the subducting plate during SSE2 (as the impermeable seal at the plate interface is broken during the SSE). Likewise, the velocity decrease that we observe along with the $\mathrm{Vp} / \mathrm{Vs}$ ratio increase as well as the delay time decrease observed by Zal et al. (2020) suggest an increase in amount of interconnected fluids in the upper plate during SSE2. After SSE2, the plate boundary becomes resealed, 
and the fluid pressure gradually builds up again beneath the low-permeability boundary (producing a decrease in the stress ratio; Warren-Smith et al., 2019; Figure 3.6b) The fluids in the upper plate dissipate over time, producing an increase in velocity and a decrease in Vp/Vs (Zal et al., 2020; Figure 3.6d). Together, these observations support the suggestion of Warren-Smith et al. (2019) that fault valve behaviour may regulate the timing and occurrence of SSEs (Figure 3.7). Although the lower plate region sampled by stress ratio changes (Warren-Smith et al., 2019) is not within the footprint of the HOBITSS II network (but closer to the coast), there may be some coming from the interface, or from the slab underneath the network, which has not been resolved with the focal mechanism catalogues (Warren-Smith et al., 2019). Although the volume and pathway of the fluids is largely unconstrained, our observation of decreased velocity is consistent with fluid migration into the upper plate during SSE2 (Zal et al., 2020).

Periods of both burst-type repeating earthquakes (Shaddox and Schwartz, 2019) and tremor (Todd et al., 2018) were observed during and after SSE2 (shown in Figure 3.6). The repeating earthquakes are suggested to occur on an upper-plate fracture network above the subducted seamount, lasting about two months. The timing of repeating earthquake activity spans both a period of velocity decrease during SSE2, and the subsequent velocity increase. A possible explanation can be found in the relative timing and location of the repeating earthquake activity. The majority of the repeating earthquakes occurred following the end of the SSE2 main slip, with Shaddox and Schwartz (2019) arguing that these, along with the tectonic tremor, were triggered by the migration of fluid from over-pressured sediments down-dip of the seamount into the upper plate fracture network. The time needed for fluids to migrate from the interface to the surface is on the order of days to weeks. We suggest that the velocity decrease we observe during SSE2 could be associated with the initial migration of fluids from the interface into the upper plate, which triggered the repeating earthquakes during SSE2. The subsequent velocity increase may be associated with diffusion of fluids through the upper plate after SSE2, which could also be responsible for triggering the repeating earthquakes. The geophysical signatures of fluid movement depend on the pathways that the fluids take and the amount of fluids migrating. Drawing direct correlations between variations in crustal velocities, repeating earthquakes, and fluid migration events remains challenging. Upper plate fluid migration models 
are required to test the timing difference.

The patterns of temporal variations of the velocity and the stress ratio for SSE3 are also broadly consistent. Before SSE3, the decrease in stress ratio implies a fluid pressure accumulation in the lower plate related to the presence of a lowpermeability seal, while an increase in velocity is caused by fluids diffusing out of the upper plate following SSE2 (Figure 3.7). Subsequently, the increase in stress ratio and the decrease in velocity start a week or two earlier than the timing of SSE3 observed from onshore GNSS stations. However, it is plausible that SSE3 began earlier in the offshore region, out of reach of the onshore GNSS stations; this supposition is consistent with the stress ratio (Warren-Smith et al., 2019), and seismic velocity changes. 

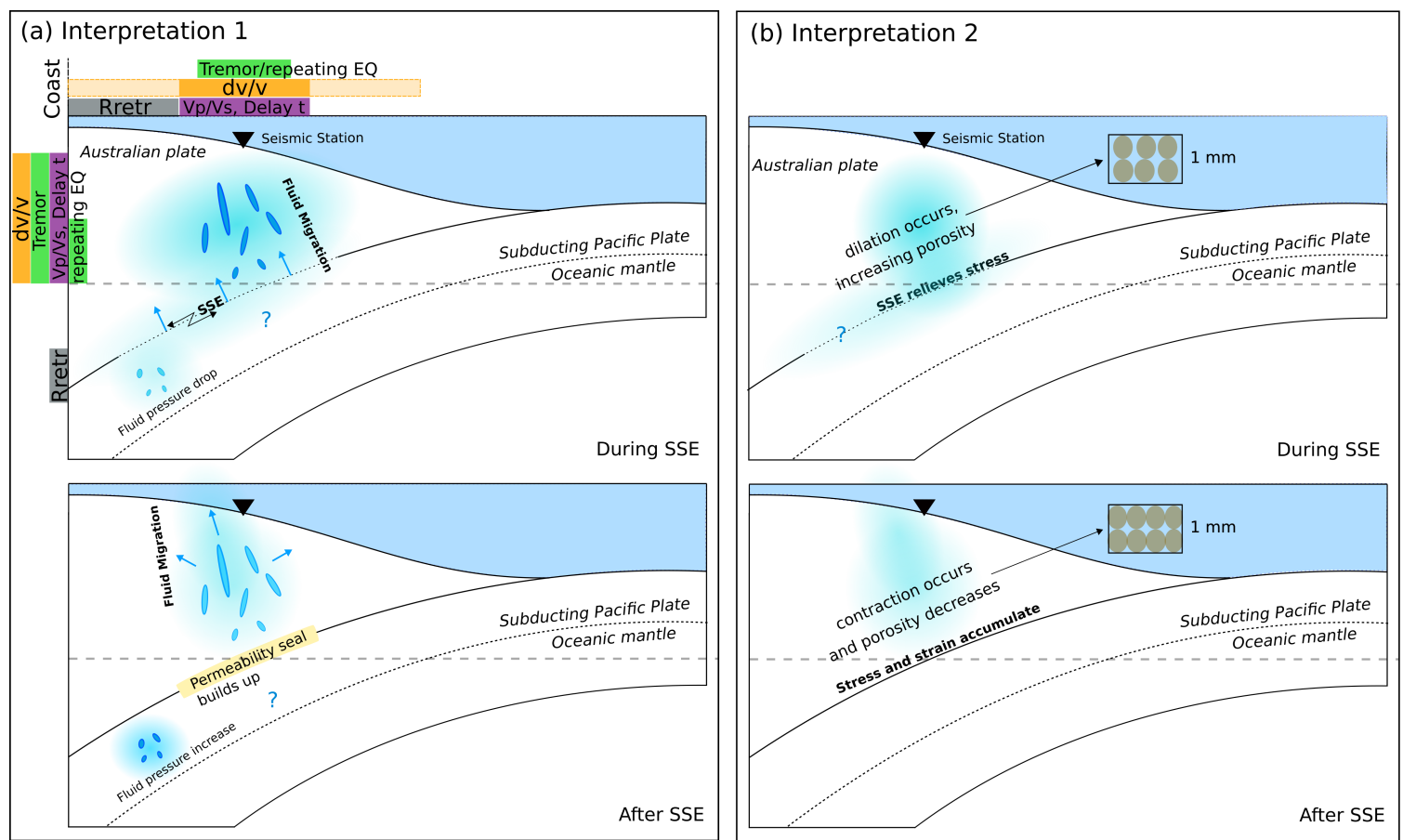

Figure 3.7: Model adapted after Husen and Kissling (2001) and Zal et al. (2020). The horizontal grey dashed lines mark the depths of our observation under the seismic station, referring to the sensitivity kernels (Figure 3.3). The bars on the top of Figure (a) show the horizontal space coverage of different data (Figure 3.6), and the bars on the left of Figure (a) show the corresponding vertical space coverage. The main observation of $\mathrm{dv} / \mathrm{v}$ is considered to be under the network (orange), though it is possible that it extends over a wider region (light orange). Here we give two interpretations. A combination of both processes is also possible. (a) Interpretation 1: Fluid migration. During the SSE, the low-permeability seal on the plate boundary is broken. This allows the interconnected fluids around the interface to migrate to the upper plate, causing a seismic velocity decrease in the upper plate. In the lower plate, Warren-Smith et al. (2019) observed a fluid pressure drop near the coast. However, we do not have constraints for the lower plate within the footprint of the HOBITSS II network. After the SSE, the fluids in the upper plate diffuse and the seismic velocity increases. The permeability seal on the plate interface establishes itself, and the fluid pressure in the lower plate starts to re-accumulate. (b) Interpretation 2: crustal strain changes through the SSE cycle. During the SSE, the occurrence of SSE relieves stress, resulting in dilation and an increase of porosity, which in turn decreases the velocity. With the increase of porosity, there might be an increase in fluid volume. After the SSE, the subduction interface re-locks and contractional elastic strain starts to accumulate and squeeze the materials, resulting in a decrease of porosity and increase in velocity between SSEs. 


\subsubsection{Assessing our observations in terms of porosity changes due to crustal strain}

The outer forearc overlying the shallow SSE region of north Hikurangi is thought to be largely composed of Miocene to recent sediments of the East Coast Basin (analogous to the Tolaga Group exposed in the adjacent onshore region (Mazengarb and Speden, 2000)), consisting of interlayered mudstones, sandstones, and occasional limestones (Neef and Bottrill, 1992; Wallace et al., 2019). According to previous studies (Gardner et al., 1974; Eberhart-Phillips et al., 1989; Bassett et al., 2014 ), the porosity in our study region is about $20 \%$ to $35 \%$. Assuming a Vs of $1.3 \mathrm{~km} / \mathrm{s}$ (Yarce et al., 2019), and based on the relationship of Vs and porosity $\phi$ (Mavko et al., 2020; Hoffman and Tobin, 2002), the velocity decreases $0.06 \%$ during SSE2 and so the effective porosity increases on the order of $0.0001 \%$, and the percentage change of porosity is about from $0.0001 \% / 35 \% \times 100 \%=0.0003 \%$ to $0.0001 \% / 20 \% \times 100 \%=0.0005 \%$. The velocity decrease can also be explained by small changes in porosity resulting from crustal strain acting on the upper plate during the SSE, which places the upper plate overlying the SSE source into dilation. The occurrence of SSE2 relieves the accumulated stress and strain recovers elastically, which results in dilation. The porosity then increases, and velocity decreases. After SSE2, the fault resumes inter-SSE locking, and the upper plate experiences contraction as elastic strain accumulates, causing the porosity to steadily decrease and velocity to increase (Figure 3.7 (b)).

Whereas we estimate the change in porosity using changes in shear-wave velocity, the elastic moduli of accretionary prism rocks can be used independently to estimate the positive volume change (dilation) expected to result from the reduction in stress coincident with an SSE. Assuming an average prism sediment Vs $=1300 \mathrm{~m} / \mathrm{s}$ (Yarce et al., 2019), a sediment density of $\rho=2300 \mathrm{~kg} / \mathrm{m}^{3}$ (EberhartPhillips et al., 2020), the shear modulus, G, is equal to approximately $4 \mathrm{GPa}$ (where $V s=\sqrt{G / \rho})$. Using an average prism sediment $\mathrm{Vp}=2300 \mathrm{~m} / \mathrm{s}$ (Yarce et al., 2019), the bulk modulus $\mathrm{K}$ is approximately $7 \mathrm{GPa}$ (where $V p=\sqrt{(K+4 G / 3) / \rho}$ ).

From the bulk modulus, we can then calculate the volume change expected for the prism sediments following slip during the SSE, which results in a stress drop given by $\delta \sigma_{\text {static }}=G d / w$ (Stein and Wysession, 2009). Where $\mathrm{G}$ is the shear modulus, $d$ is the amount of slip, and $w$ is the down-dip width of the fault. For the 
displacement $(\mathrm{d}=0.27 \mathrm{~m})$ and down-dip width of the fault $(\mathrm{w}=33000 \mathrm{~m})$ measured in SSE2 (Wallace et al., 2016), $\delta \sigma_{\text {static }}$ is estimated to be $30 \mathrm{kPa}$. A $30 \mathrm{kPa}$ stress drop equates with a percent volume change of $0.0004 \%$ using the relationship $K=\delta \sigma_{\text {static }} /(\delta V / V)$. This volume change, derived from the estimated stress drop and measured bulk modulus of the hanging wall sediments, concurs with the percent change in porosity $(0.0003 \%$ to $0.0005 \%)$ estimated from seismic velocity variations. We propose that given sufficient resolution, seismic velocity variations may be capable of monitoring minute strains in the hanging wall of subduction zone megathrusts.

Rivet et al. (2011) studied velocity variations at 7-17 s, corresponding to the depth of 5-20 km (upper and middle crust), related to the 2006 Mexico SSE. Following this, Rivet et al. (2014) observed velocity variations at 12-24 s, corresponding to the depth of 10-30 km (middle and lower crust), related to the 2009-2010 Mexico SSE. They discussed that there are possible velocity variations at longer periods and deeper depths (Rivet et al., 2014). Table 3.1 compares the velocity variations associated with the 2006 and 2009-2010 Mexico SSEs (Rivet et al., 2011, 2014) with this study.

Rivet et al. (2011) computed the static strain field associated with the 2006 Mexico SSE which showed that the SSE produced an extended increase in dilation. This coincides with our interpretation of crustal strain changes through the SSE cycle. However, volumetric deformation $\left(10^{6}\right)$ estimated during the 2006 Mexico SSE was much smaller than the volume change that they estimated $\left(10^{3}\right)$ from their velocity change, suggesting that the velocity change was related to the strain rate rather than the strain itself (Rivet et al., 2011). The finding from Rivet et al. (2014) for the 2009-2010 Mexico SSE supported this conclusion. During the 2014 Gisborne SSE2 in this study, however, our estimated percent volume change $(0.0004 \%)$, derived from the estimated stress drop and bulk modulus of the hanging wall sediments, concurs with the percent porosity change $(0.0003 \%$ to $0.0005 \%)$ estimated from seismic velocity variations. Therefore, if the observed velocity change is due to a change in porosity, we suggest it is related to strain. Rivet et al. (2014) found significant correlation between tremor and velocity variations during the SSE, suggesting that these two independent observations can be linked to the same mechanism. For the 2014 Gisborne SSE2, however, both the tremor and repeating earthquakes occurred slightly later than the start of SSE2 and the measured velocity decrease. 
Table 3.1: Comparison of Rivet et al. (2011) and this study.

\begin{tabular}{|l|l|l|l|}
\hline & $\begin{array}{l}\mathrm{dv} / \mathrm{v} \text { for 2014 Gis- } \\
\text { borne SSE2 }\end{array}$ & $\begin{array}{l}\mathrm{dv} / \mathrm{v} \text { for 2006 } \\
\text { Mexico SSE }\end{array}$ & $\begin{array}{l}\mathrm{dv} / \mathrm{v} \text { for 2009- } \\
2010 \mathrm{Mexico} S \mathrm{SE}\end{array}$ \\
\hline periods & $2.5-6 \mathrm{~s}$ & $7-17 \mathrm{~s}$ & $>12 \mathrm{~s}$ \\
\hline depth & $\begin{array}{l}<\mathrm{km} \text { (crust in } \\
\text { upper plate) }\end{array}$ & $\begin{array}{l}5-20 \mathrm{~km} \mathrm{(upper} \\
\text { and middle crust) }\end{array}$ & $\begin{array}{l}>10 \mathrm{~km} \text { (middle } \\
\text { and lower crust) }\end{array}$ \\
\hline volume change & $\begin{array}{l}\text { same order as the } \\
\text { estimation using } \\
\mathrm{dv} / \mathrm{v}\end{array}$ & $\begin{array}{l}\text { much smaller than } \\
\mathrm{dv} / \mathrm{v}\end{array}$ & $\begin{array}{l}\text { much smaller than } \\
\mathrm{dv} / \mathrm{v}\end{array}$ \\
\hline relate to & strain & strain rate & strain rate \\
\hline
\end{tabular}

This is particularly obvious for the repeating earthquakes, which occurred until the velocity increased to its peak. A possibility is that the velocity variations are either partly or fully controlled by the strain/volume changes. Fluid migration, which can trigger the tremor and repeating earthquakes, may happen later or over a longer time period.

\subsection{Conclusions}

We analyse one-year ambient noise data acquired by nine OBSs deployed in the northern Hikurangi Margin in a region where SSEs occur. During the deployment from May of 2014 to June of 2015, there are four SSEs (Warren-Smith et al., 2019), with the strongest of them (SSE2) occurring from September to October 2014, lasting five weeks. We compute temporal velocity variations using coda waves retrieved from single station cross component correlations. The average velocity variations exhibit a velocity decease during SSE2 and a velocity increase after SSE2. The velocity variations fit well with the variations of stress ratio, $\mathrm{Vp} / \mathrm{Vs}$, and shear-wave splitting delay times from other studies (Warren-Smith et al., 2019; Zal et al., 2020; Shaddox and Schwartz, 2019; Todd et al., 2018). After SSE2, the velocity decrease followed by an increase returning to original values might be caused by SSE3.

We give two possible end-member interpretations: 1) Fluid migration related to fault-valve behavior between the two plates: Before SSE2, there is a permeability seal on the plate boundary that traps fluid beneath the subduction interface and 
fluid pressure steadily increases, to the point at which the SSE is triggered (WarrenSmith et al., 2019). The occurrence of SSE2 breaks the seal on the plate boundary and trapped fluids migrate to the upper plate. The fluid migration takes place over approximately one month from late September to the start of November 2014. We observe a velocity decrease in the upper plate consistent with increased fluid volume in the upper plate. After SSE2, the plate boundary re-seals and the fluids in the upper plate diffuse, and velocity begins increasing again. This happens after SSE2 until around 1st December 2014. This interpretation is consistent with that of Warren-Smith et al. (2019) and Zal et al. (2020). 2) Crustal strain changes through the SSE cycle: Before SSE2, the plates are locked together along the plate interface, and elastic strain (largely contraction) accrues in the overriding plate. The occurrence of SSE2 relieves the accumulated elastic strain, resulting in dilation and an increase of porosity. This takes place over approximately one month from late-September to the start of November 2014. We observe a velocity decrease in the upper plate, which may be consistent with increased porosity. A porosity increase can lead to an increase of fluid volume. After SSE2, the plate boundary re-locks and contraction begins again, resulting in a subsequent porosity decrease and velocity increase. This happens after SSE2 until around 1st December 2014.

We point out that aspects of both the interpretations may be at play to explain the observed seismic velocity changes. Both of them can possibly lead to fluid volume changes, but the volume and pathway of the fluids are unconstrained. A combination of fluid migration related to fault-valve behavior and consequences of crustal strain during the SSE may explain both this study and previous research (WarrenSmith et al., 2019; Zal et al., 2020; Shaddox and Schwartz, 2019; Todd et al., 2018). Our study shows that velocity variations associated with the occurrence of SSEs in New Zealand are detectable using ambient noise interferometry, and results provide new evidence to support the integral role that fluids and accumulated elastic strain energy play in promoting slow slip events on the northern Hikurangi Margin.

\subsection{Acknowledgments}

This research was supported by an Endeavour Fund grant (funding contract C05X1605) from Ministry of Business, Innovation, and Employment. Additional funding sup- 
port came from National Science Foundation (NSF) grants: OCE-1333311, OCE1551683, OCE-1333025, OCE-1334654, and OCE-1551922. The ocean bottom seismic data was provided by instruments from the Ocean Bottom Seismograph Instrument Pool (www.obsip.org) funded by the National Science Foundation (NSF), the Earthquake Research Institute (ERI), University of Tokyo, and New Zealand's GeoNet project. Raw data from the experiment is archived at Incorporate Research Institutions for Seismology Data Management Center (IRIS-DMC) with experiment codes YH 2014-15 (seismic data) and 8F 2014-15 (bottom pressure record data).

We acknowledge the HOBITSS II project, and Tangaroa cruise TAN1405 to collect the data. We acknowledge MSNoise 1.5, which supports this study technically. We also acknowledge Pasan Herath, Katherine Woods, Katrina Jacobs, Susan Schwartz, and Heather Shaddox for their helpful feedback and discussion. 


\section{Chapter 4}

\section{Temporal velocity variations in 2018-2019 in the northern Hikurangi margin and the relation to slow slip}

\subsection{Abstract}

Seismic velocity variations have been observed in association with SSEs (Chapter 3). This chapter uses different data set to test if the observation is consistent, as well as the hypotheses proposed by previous observation. From the study of an SSE occurring from September to October 2014, seismic velocity decreases during the SSE and increases after the SSE. Two hypotheses are proposed: 1) the velocity decrease during the SSE is caused by more fluids migrating into the upper plate as the SSE breaks a low-permeability seal on the plate boundary. 2) the velocity decrease during the SSE is caused by increased porosity because the SSE relieves the accumulated elastic strain on the subduction interface, which results in dilation. As a part of the Hikurangi Ocean Bottom Investigation of Tremor and Slow Slip (HOBITSS V) project, five OBSs were deployed for one year from 2018 to 2019. An SSE occurred with the largest slip of $200 \mathrm{~mm}$ during the deployment, from the end of March to the beginning of May 2019. The main slip was south of the deployment, and the slip beneath the deployment was up to $150 \mathrm{~mm}$. This chapter uses the ambient noise data recorded by the five OBSs to compute velocity variations in the upper crust of the overriding plate. The average velocity variations show a 
decrease on the order of $0.015 \%$ during the SSE and an increase back to the original velocity value after the SSE, which is consistent with the observation in Chapter 3. Velocity variations computed from individual stations also show velocity increases before the SSE, which are suppressed on the average velocity variations through destructive interference because of their time difference. The velocity increases before the SSE can only be explained by the elastic strain changes (the 2nd hypothesis) if the velocity increases from individual stations are real, but the 1st hypothesis (fluid migration), suggested by previous studies using different geophysical data set (stress ratio and $\mathrm{Vp} / \mathrm{Vs}$ ), can still happen at the same time.

\subsection{Introduction}

In the northern Hikurangi margin, some of the world's shallowest slow slip events (SSEs, $<10 \mathrm{~km}$ deep) are well-documented (Wallace et al., 2016). In 2014-2015, during the Hikurangi Ocean Bottom Investigation of Tremor and Slow Slip II (HOBITSS V II) deployment, four SSEs occurred and were recorded successfully, from which the second SSE (SSE2) had the most significant slip (over $250 \mathrm{~mm}$ ). In Chapter 3 , ambient noise data recorded by nine OBSs deployed by the HOBITSS V II are used to calculate single station cross-component correlations and compute seismic velocity variations. The velocity shows a decrease on the order of $0.05 \%$ during SSE2, followed by an increase of similar magnitude afterwards. Two possible interpretations are proposed: 1) Fluid migration related to fault-valve behavior between the two plates: Before SSE2, there is a permeability seal on the plate boundary that traps fluid beneath the subduction interface, and fluid pressure steadily increases (Warren-Smith et al., 2019). The occurrence of SSE2 breaks the seal on the plate boundary, and the trapped fluids migrate to the upper plate. A velocity decrease in the upper plate is consistent with increased fluid volume in the upper plate. After SSE2, the plate boundary re-seals, and the fluids in the upper plate diffuse, and velocity begins increasing again. This interpretation is consistent with that of Warren-Smith et al. (2019) and Zal et al. (2020). 2) Crustal strain changes through the SSE cycle: Before SSE2, the plates are locked together along with the plate interface, and elastic strain (largely contraction) accrues in the overriding plate. The occurrence of SSE2 relieves the accumulated elastic strain, resulting in dilation and an increase of porosity. A velocity decrease in the upper plate is consistent with increased porosity. A porosity increase also possibly lead to a fluid 
volume increase. After SSE2, the plate boundary re-locks, and contraction begins again, resulting in a subsequent porosity decrease and velocity increase.

An SSE from 3rd March 2019 to 6th July 2019 includes the brief Porangahau slow slip at the beginning, the Gisborne slow slip in March and April, and the Hawke Bay slow slip that continues into June (Woods et al., 2020). This SSE was recorded successfully by five OBSs deployed by the HOBITSS V deployment from 2018 to 2019 (Barker, 2019). This chapter uses the ambient noise data recorded from 2018 to 2019 by the five OBSs to study the seismic velocity variations in the upper crust of the overriding plate related to the SSE in 2019 and provides more evidence to support and improve the hypothesis proposed in Chapter 3.

\subsection{Data}

From October 2018 to October 2019, for the HOBITSS V deployment (Barker, 2019), five short-period OBSs with a natural period of $1 \mathrm{~s}$ from Earthquake Research Institute, Japan (EOBS), were deployed offshore Gisborne (Figure 4.1). The OBSs were deployed on the overlying Australian plate to record SSE data. The OBSs deployed from 2014 to 2015 (Chapter 3) are also plotted in Figure 4.1 for reference.

The continuous data recorded by three-component seismometers of the five OBSs (green triangles in Figure 4.1) are used to compute the single station cross-component correlation functions.

There are three components equipped on an OBS: two horizontal components, 1 and 2, and a vertical component. In contrast to the 2014-2015 data process, horizontal components of the stations are not rotated because the orientations are not determined. Therefore, they are called 1 and 2 instead of North and East. Coda waves are derived from vertical-horizontal 1 cross-correlations, vertical-horizontal 2 cross-correlations, and horizontal1-horizontal2 cross-correlations, to compute velocity variations. 


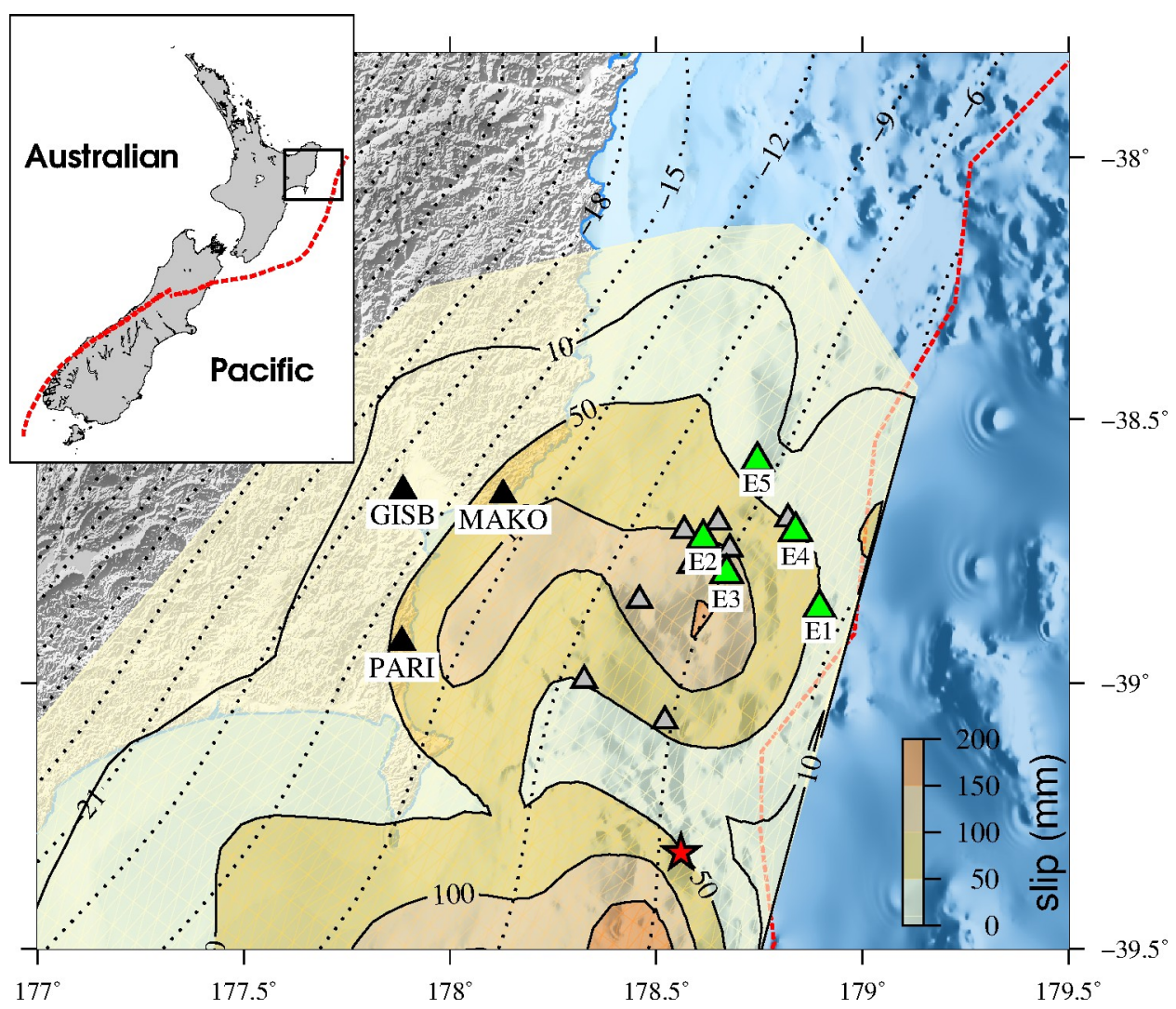

Figure 4.1: Locations of ocean bottom seismometers deployed in 2018-2019 (Barker, 2019), with the contours showing slip (Woods et al., 2020) of an SSE occurring from the end of March to May 2019. Station names are shortened from EOBS to E. The green triangles denote the stations used in this chapter, and the grey triangles denote the stations deployed in 2014-2015 used in Chapter 3. Black triangles show the on-land GNSS stations. The red star denotes a magnitude 5 earthquake occurring on 14th May 2019. The dashed red line shows the plate boundary interface (Coffin et al., 1998). Dotted contours show plate interface depths in km (Williams et al., 2013).

\subsection{Methods}

\subsubsection{Parameterization}

Similar to Chapter 3, parameters in MSNoise are tested. Different filter ranges are tested and compared with lag-time dependent SNR. Figure 4.2 shows the SNR averaged from all the components of all the stations. Except for the filter of 10-20 s, all the filters' cross-correlations have enough SNR $(>2)$ for velocity variation computation. To compare with the results in Chapter 3, filters 1-2.5 s and 2.5-20 s are used for cross-correlation computation. Because the instrument type is the same as the EOBSs deployed in 2014-2015 and the general geometry is the same, param- 
eters of segmentation length (raw data window length to correlate), segmentation overlap (overlap in window length between segments), normalization (1-bit, or windsorizing at $\mathrm{N}$ time Root-Mean-Square) are kept the same as the parameters used in Chapter 3 for EOBSs.

A large size of the current stack can decrease the temporal resolution and might hide some velocity changes happening in a short time, but too small a size of the stack might not be enough to suppress noise. The current stack size is determined to be 20 days according to the correlation coefficients between current stacks and the reference stack (Figures 4.3 and 4.4). In contrast to Chapter 3, the correlation coefficients between the current stacks and the reference stack are all high, and therefore, no threshold is set for selecting single-day stacks before stacking for current stacks and the reference stack.

The delay time between the current stacks and the reference stack is easier to identify on the coda than on the direct arrivals because coda waves travel longer distances. The coda window on the cross-correlations to compute velocity variations is determined by the lag-time dependent SNR (Figures 4.5 and 4.6), with the SNR of all the single stations larger than 2. The SNR threshold follows that of previous studies (Yates, 2018; Yates et al., 2019). The coherence threshold is determined by the relationship between SNR and coherence to exclude the data with $\mathrm{SNR}<2$. A current stack must be similar to the reference stack to ensure its quality. Coherence between a current stack and the reference stack is computed and a threshold of coherence is set to 0.89 to exclude noisy current stacks (Figures 4.5 and 4.6). The coda window on the negative lag is set to be $-70 \mathrm{~s}$ according to the SNR. The window on positive lag is set to be the same (70 s) to keep it symmetric. Direct arrivals are excluded (-20 s to $20 \mathrm{~s})$. 

slip

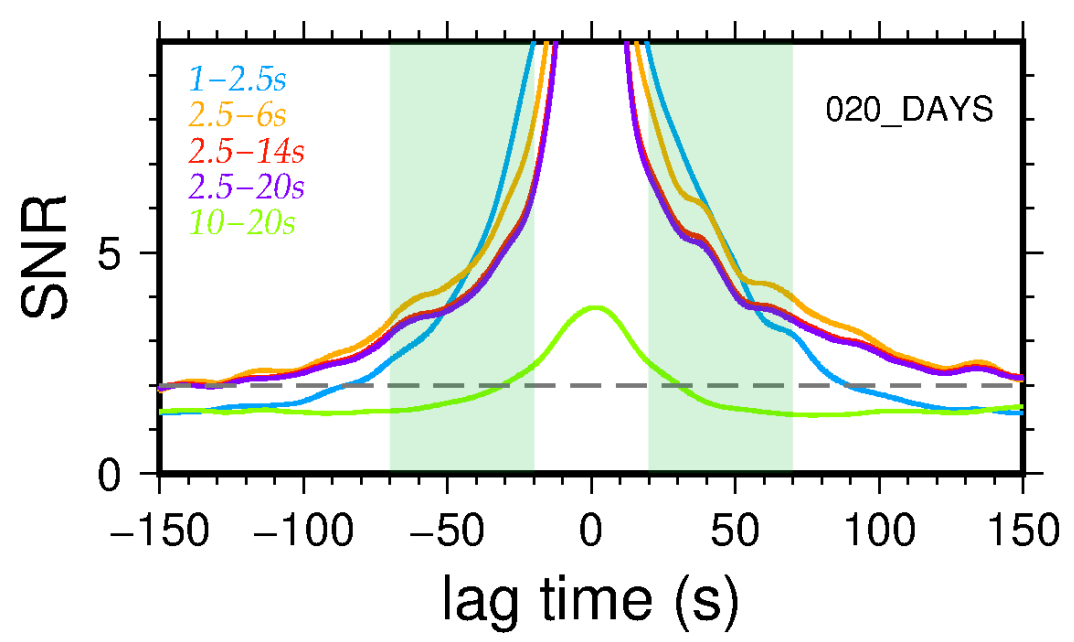

Figure 4.2: Comparison of different filters by the SNR averaged from all the components of all the stations. The curves of SNR are color-coded by the filter period bands. 20-day stacks are used. The green shades mark the coda window to compute velocity variations, and the horizontal dashed line marks SNR=2. The cross-correlations at 2.5-14 s (red) and 2.5-20 s (purple) have very similar SNR.

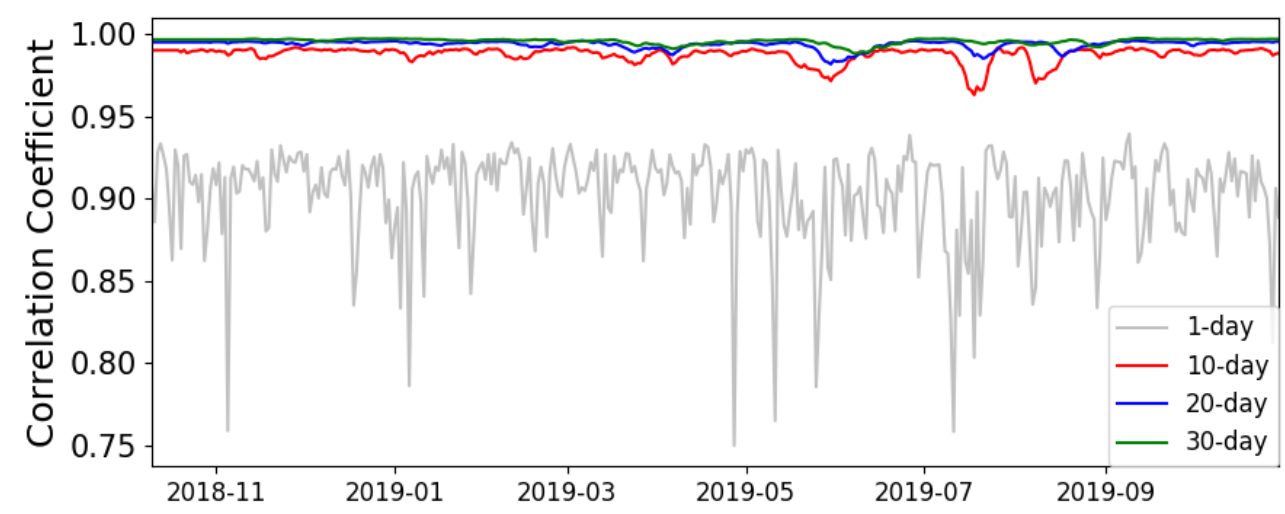

Figure 4.3: An example of the relationship between stack sizes and correlation coefficients computed on EOBS2 vertical-horizontal1 component correlations at 1-2.5 s band. Correlation coefficients between different size current stacks (1-day stacks, 10-day stacks, 20-day stacks, and 30-day stacks) and reference stack are computed and compared. From 20-day stacks to 30-day stacks, the correlation coefficient is not improved much. In contrast to Chapter 3, the correlation coefficients of 1-day current stacks are all high; no threshold of correlation coefficients is set to exclude daily cross-correlations. 


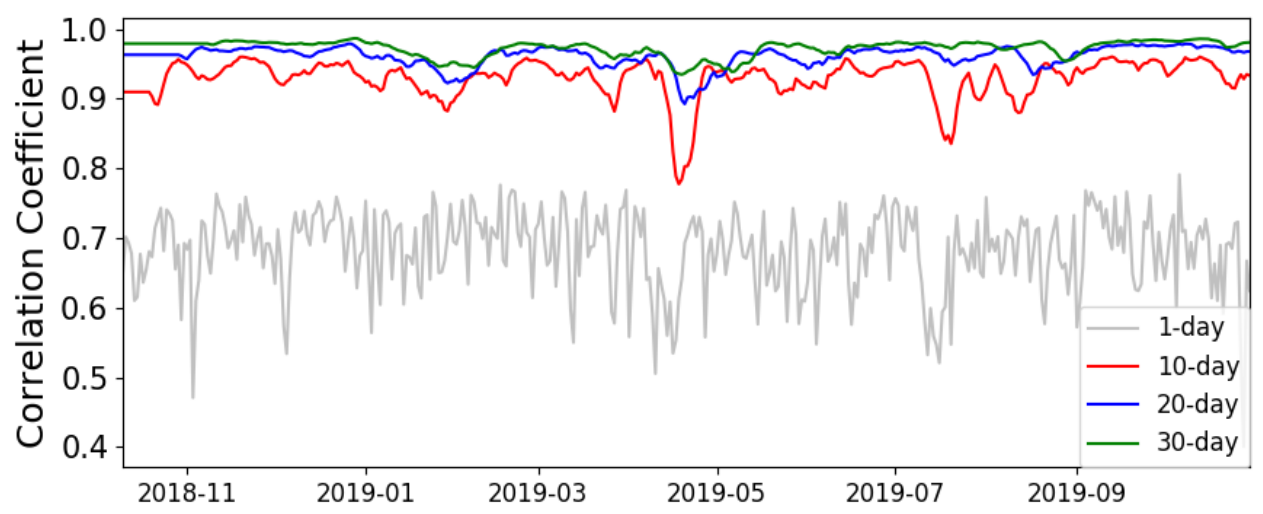

Figure 4.4: Similar to Figure 4.3 but for the 2.5-20 s band. An example of the relationship between stack sizes and correlation coefficients computed on EOBS2 vertical-horizontal1 component correlations at 2.5-20 s band. From 20-day stacks to 30-day stacks, the correlation coefficient is not improved much.
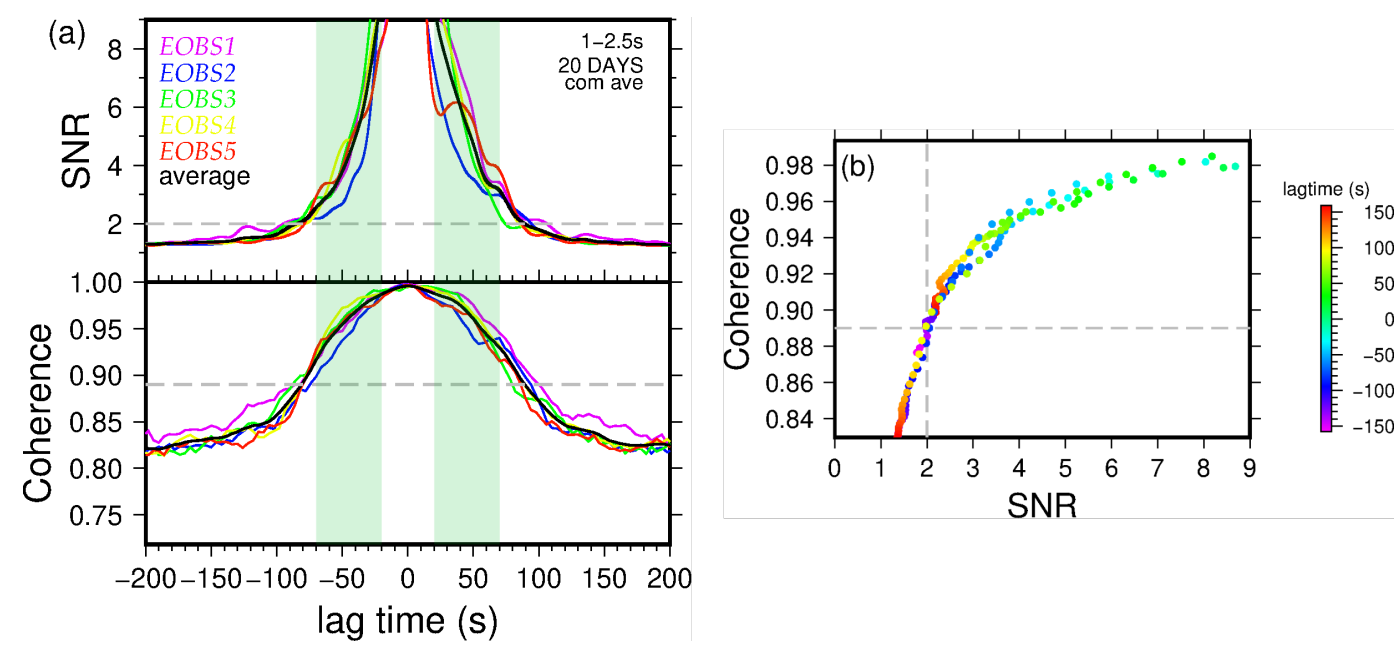

Figure 4.5: Determination of coda wave window and threshold of coherence for 1-2.5 s filter. (a) Lag-time dependent SNR and the coherence between 20-day current stacks and the reference stack of single stations (color-coded) and their average (black). For each station, the SNR is averaged from its three components. The SNR of each component is averaged from all the 20-day current stacks. The green shades mark the coda wave windows to compute $\mathrm{dv} / \mathrm{v}$, determined by the SNR of all the single stations above 2 . The horizontal dashed line on the top figure marks the threshold of SNR, and the one on the bottom figure marks the threshold of coherence. (b) Relationship between the average SNR and coherence (black curves in (a)). Different colors denote the values at different lag times. The threshold of coherence (0.89) is determined by SNR $>2$. The dashed lines mark the thresholds of SNR and coherence. 

slip

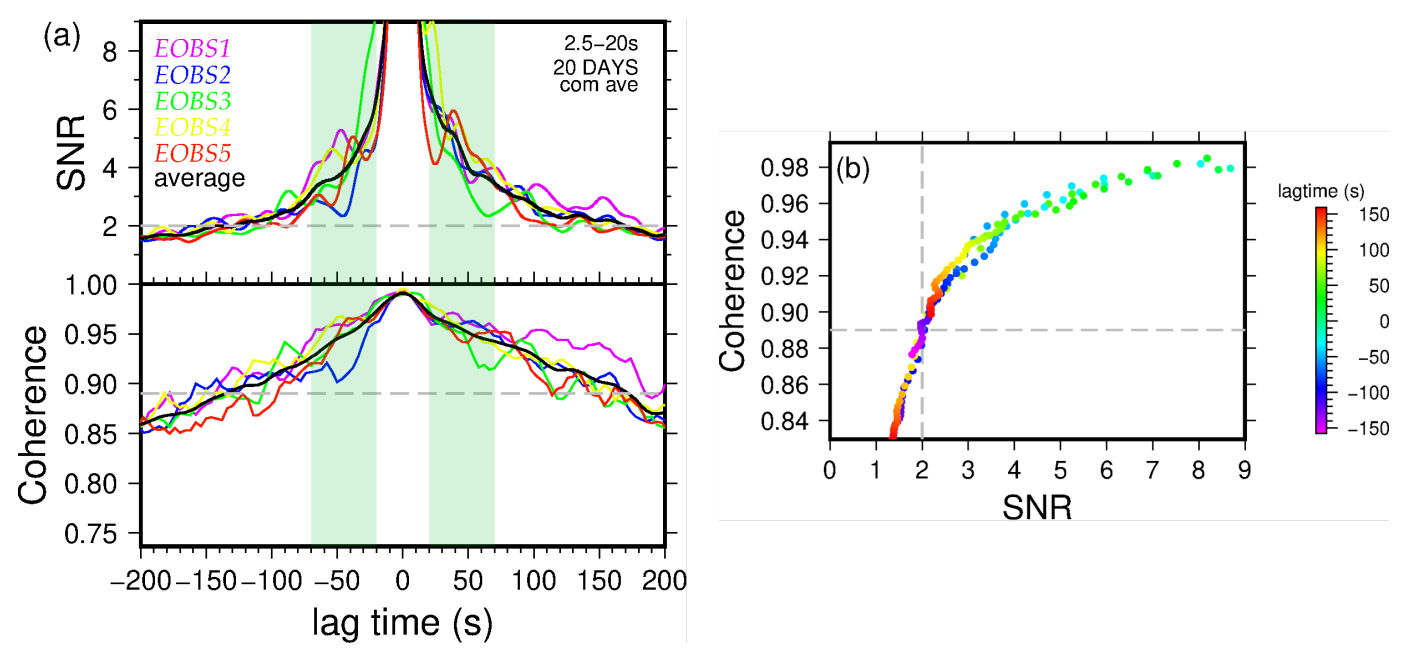

Figure 4.6: Similar to Figure 4.5 but for filter 2.5-20 s. 
Table 4.1: Final parameters used in this chapter for MSNoise 1.5

\begin{tabular}{|l|l|l|}
\hline Parameter & Description & Value \\
\hline startdate & start date for computation & $2018-10-10$ \\
enddate & end date for computation & $2019-10-30$ \\
ref_begin & start date of reference stack & $2018-10-10$ \\
ref_end & end date of reference stack & $2019-10-30$ \\
maxlag & maximum lag of cross-correlations & 300 \\
cc_sampling_rate & sampling rate for the cross-correlation & 20 \\
preprocess_lowpass & preprocessing low-pass filter & $2.0 \mathrm{~Hz}$ \\
preprocess_highpass & preprocessing high-pass filter & $0.02 \mathrm{~Hz}$ \\
remove_response & remove instrument response & $\mathrm{N}$ \\
corr_duration & data windows to correlate & $1800 \mathrm{~s}$ \\
overlap & overlap between data windows & 0.7 \\
windsorizing & windorizing at N times RMS & 3 \\
whitening & whiten Traces before cross-correlation & A (all traces) \\
stack_method & linear or phase weighted stack & linear \\
autocorr & compute single station or not & Y \\
mov_stack & current stack size & 20 days \\
mwcs_wlen & window length to perform MWCS & $20 \mathrm{~s}$ \\
mwcs_step & step of the moving window in MWCS & $4 \mathrm{~s}$ \\
dtt_lag & how the window is defined & static \\
dtt_minlag & min lag time to compute dtt & $0 \mathrm{~s}$ \\
dtt_width & window length to compute dtt & $70 \mathrm{~s}$ \\
dtt_sides & which sides of stacks to use & both \\
dtt_mincoh & threshold of coherence for data & 0.89 \\
dtt_maxdt & maximum dt measurement & $0.2 \mathrm{~s}$ \\
dtt_maxerr & maximum error on dt measurement & $0.1 \mathrm{~s}$ \\
\hline
\end{tabular}




\subsubsection{Data processing}

Following the workflow in Chapter 3, data recorded on three-component channels of each station are downsampled to $20 \mathrm{~Hz}$. Two bandpass filters, 1-2.5 s, and 2.5-20 s are applied. Afterwards, time-domain normalization through clipping data at three times the Root-Mean-Square and spectral whitening is applied. Daily series are then cut to 1800 s segments with $70 \%$ overlap in window length to correlate. The cross-correlations are then linearly stacked to determine daily crosscorrelations. In Chapter 3, the correlation coefficient between each daily stack and the stack of all days is computed. The daily stacks with low correlation coefficients are excluded. However, in this chapter, the correlation coefficients are high for all the stations' daily stacks. Therefore, all the daily stacks are used. For each station, 20-day successive current stacks and a reference stack are then computed. Current stack examples and all the reference stacks are shown from Figures 4.7-4.10.

The moving-window cross-spectral analysis method (Poupinet et al., 1984; Clarke et al., 2011) is applied to measure the time differences between the current stacks and the reference stack. The measurement is carried out in the frequency domain in a series of 20-second windows with a 4-second moving step within the coda wave window. The data in a frequency band of 2.5-14 s are used when applying the moving-window cross-spectral analysis method on the 2.5-20 s stacks. The data in a frequency band of 1-2.5 s are used when applying the moving-window cross-spectral analysis method on the 1-2.5 s stacks. The relative velocity difference between the current stacks and the reference stack is then determined by $\mathrm{dv} / \mathrm{v}=-\mathrm{dt} / \mathrm{t}$ (Section 2.5), and corresponding errors are estimated (Lecocq et al., 2014). For a single station, $\mathrm{dv} / \mathrm{v}$ from the three cross-components is computed and averaged, using the median and mean values. Component-averaged $\mathrm{dv} / \mathrm{v}$ on single stations are then all averaged. The errors of the average $\mathrm{dv} / \mathrm{v}$ are estimated using a bootstrap method that calculates $95 \%$ confidence intervals for a given sample of a population and a statistic. The original data (individual station $\mathrm{dv} / \mathrm{v}$ values) are resampled with 200 times iterations. In each iteration, the sample size is the same as original data size (the number of individual stations). The samplings after 200 times iterations are then ranked from the minimum to the maximum. Afterwards, the lower 95\% confidence limit and the upper 95\% confidence limit are determined from the sampling array. The lower $95 \%$ confidence 
limit is the $l$-th percentile of the array elements, where $l$ is given by

$$
l=((1-\text { confidence }) / 2) * 100
$$

The upper $95 \%$ confidence limit is the $u$-th percentile of the array elements, where $u$ is given by

$$
u=(\text { confidence }+((1-\text { confidence }) / 2)) * 100
$$

Where confidence is $95 \%$. 


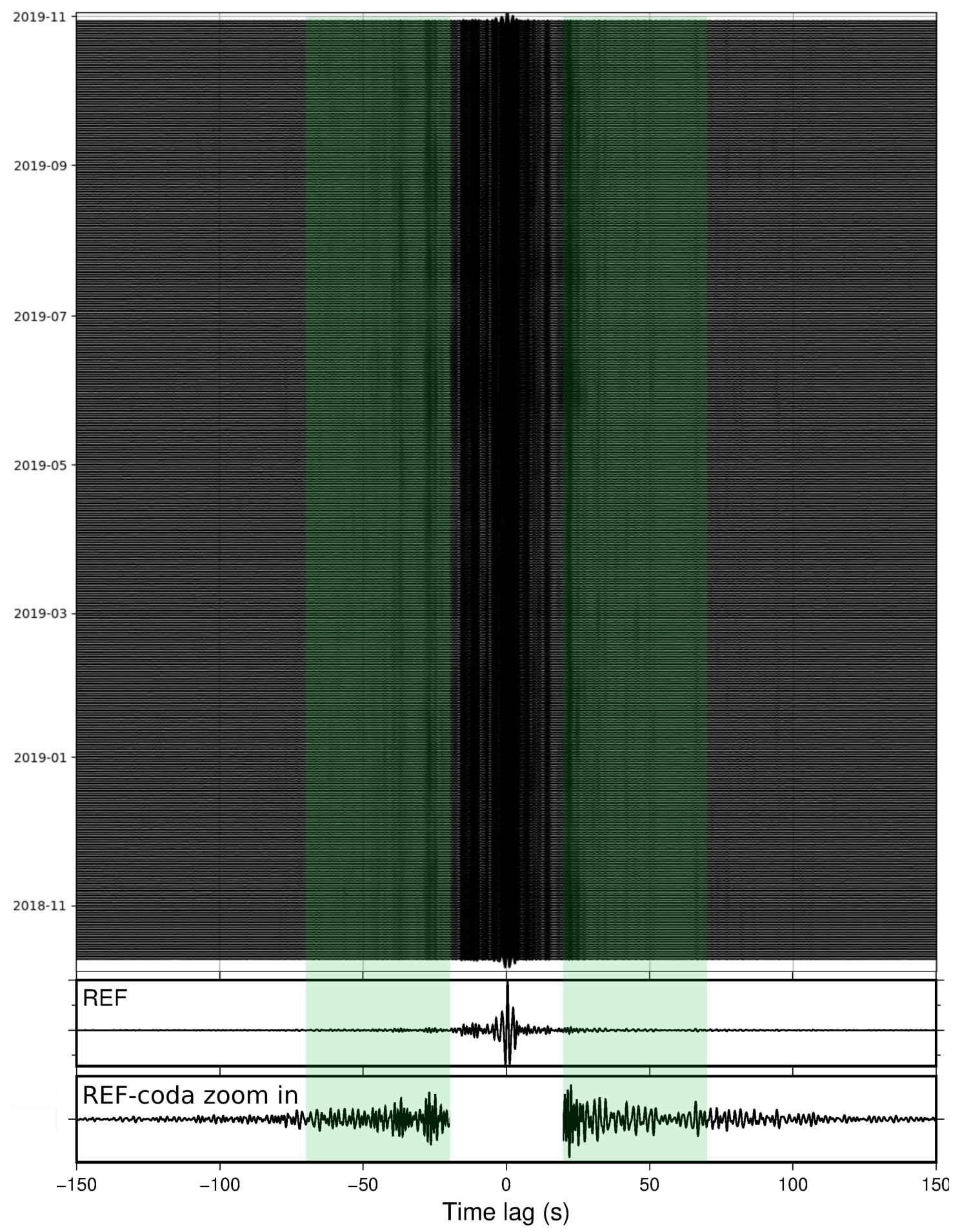

Figure 4.7: 20-day current stacks over time from EOBS2 vertical-horizontal1 component correlations and corresponding reference stack (REF), at 1-2.5 s. The bottom figure excludes the direct arrival and zooms in on the coda waves. The green shades mark the windows to compute velocity variations. 


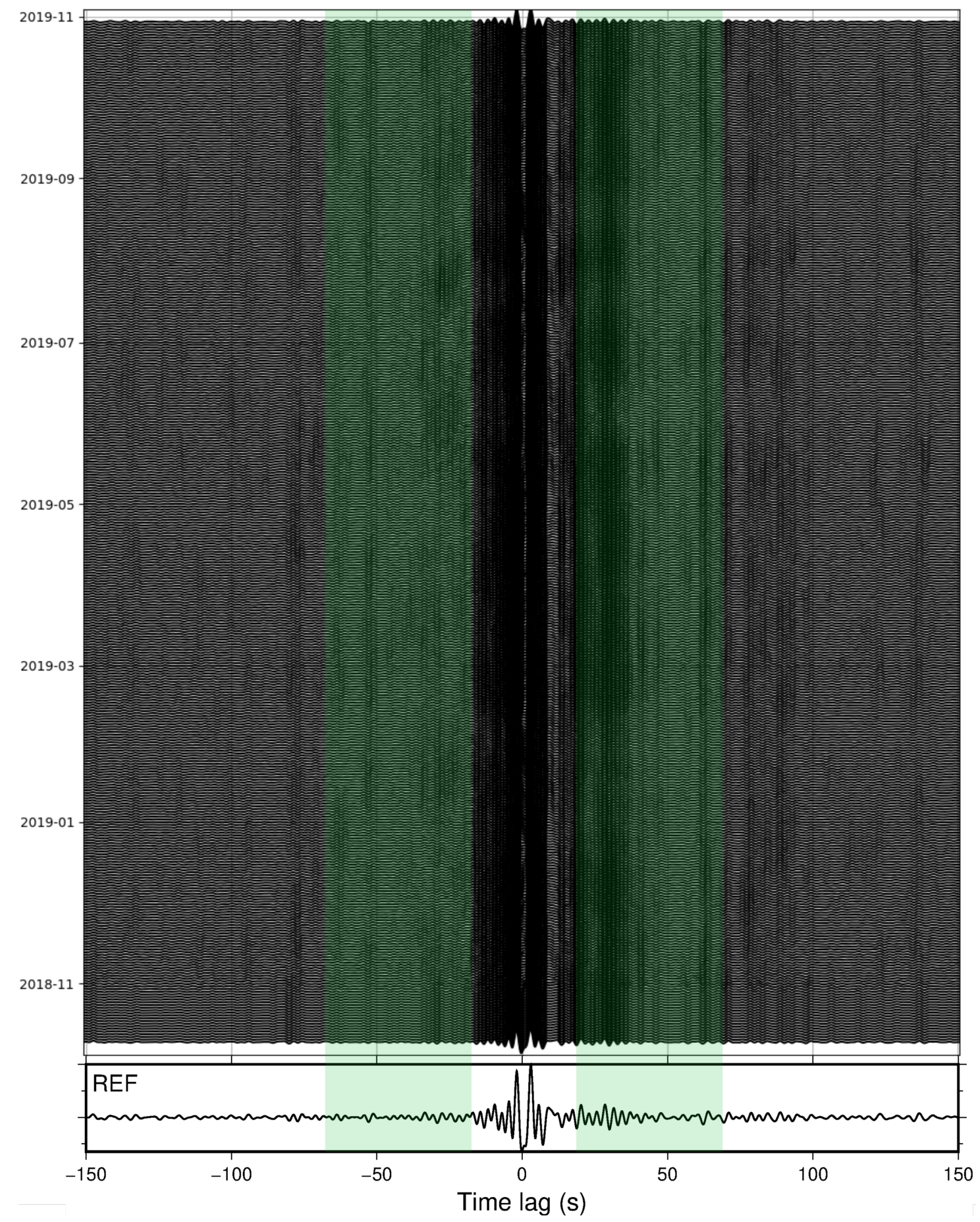

Figure 4.8: 20-day current stacks over time from EOBS2 vertical-horizontal1 component correlations and corresponding reference stack (REF), at 2.5-20 s. The green shades mark the windows to compute velocity variations. 

slip
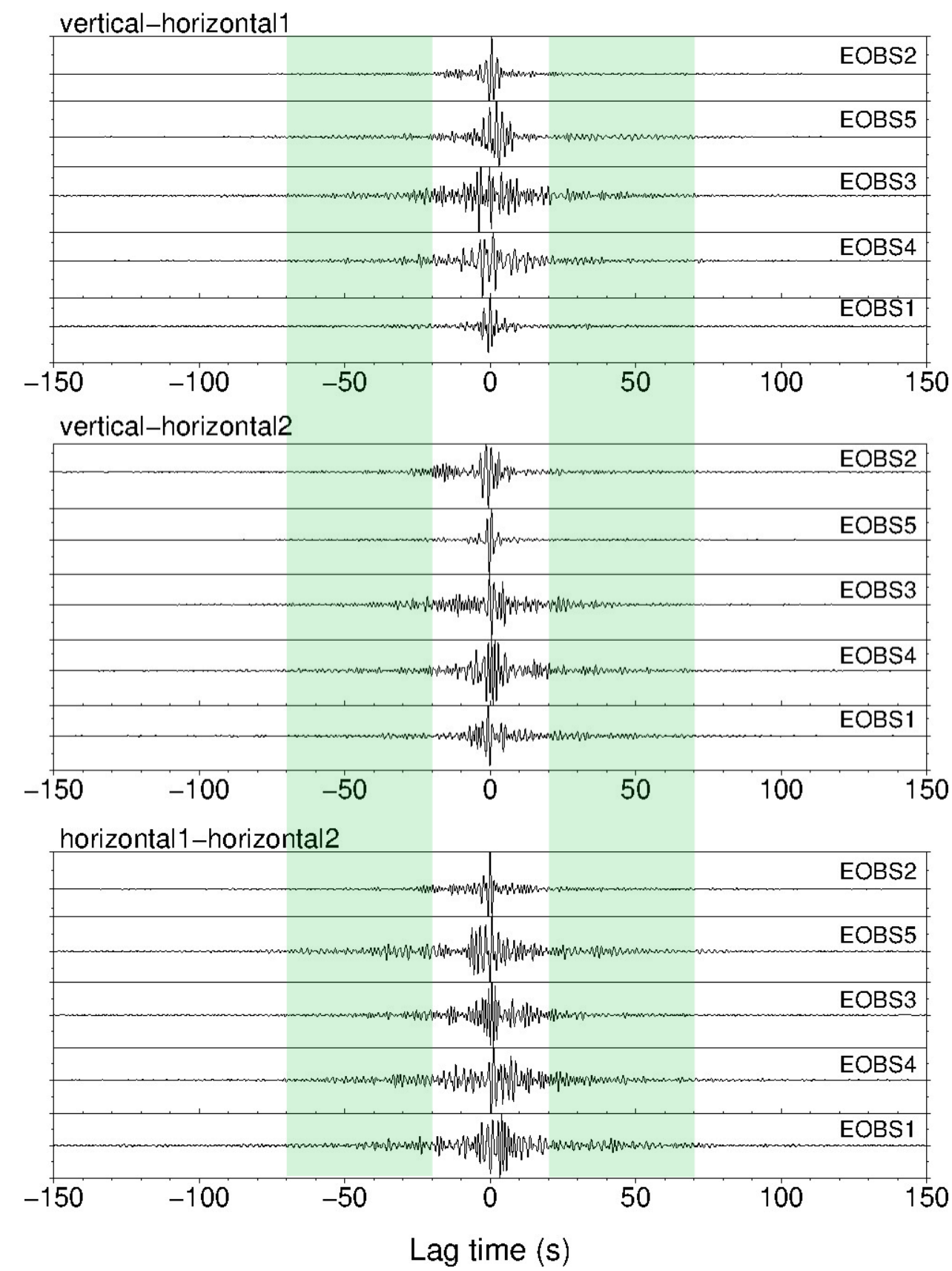

Figure 4.9: Reference stacks of $1-2.5 \mathrm{~s}$ on the three components, plotting from the closest to the coast to further (top to bottom). The green shades mark the windows used to compute $\mathrm{dv} / \mathrm{v}$. 


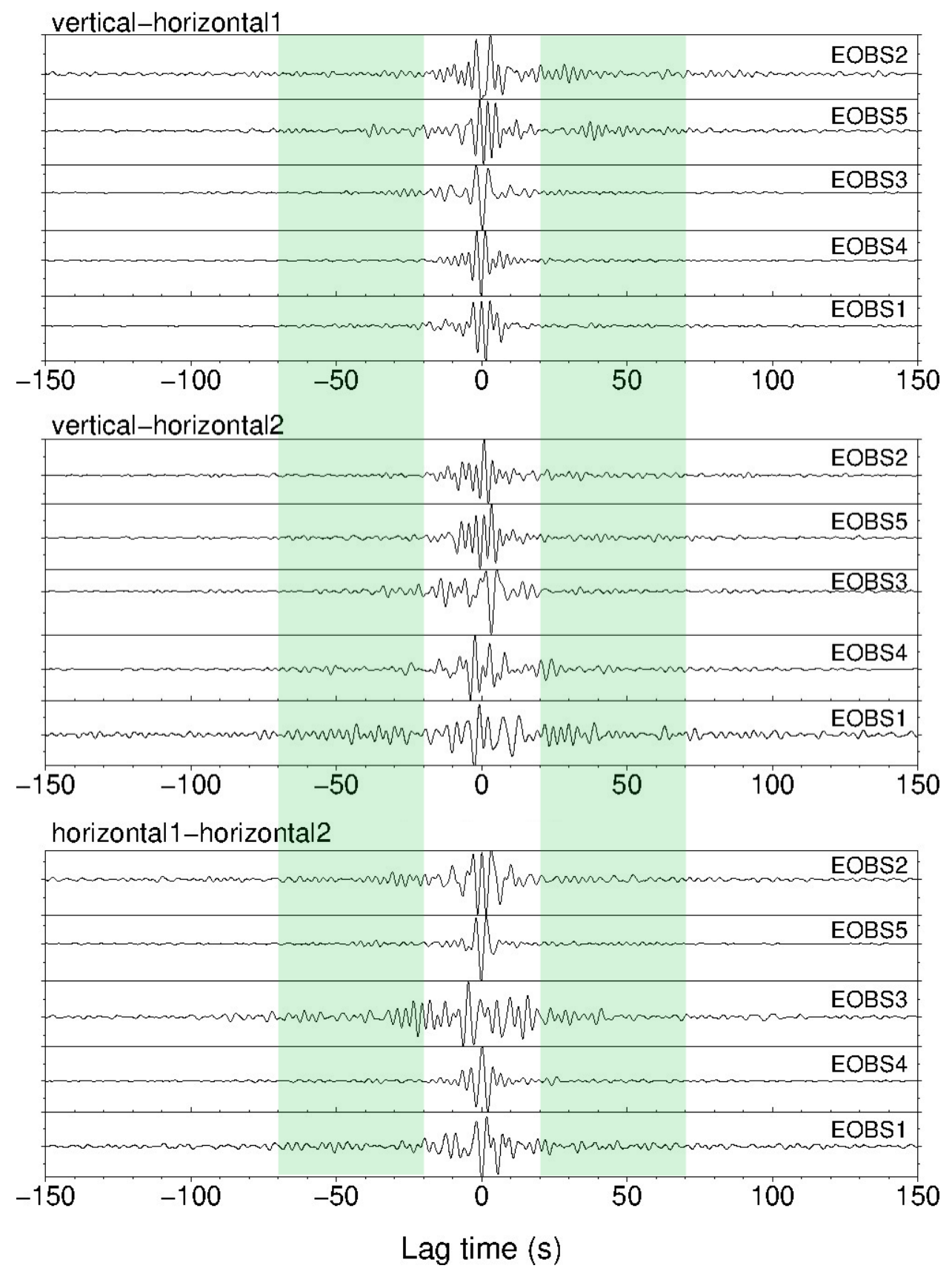

Figure 4.10: Reference stacks of $2.5-20 \mathrm{~s}$ on the three components, plotting from the closet to the coast to further (top to bottom). The green shades mark the windows used to compute $\mathrm{dv} / \mathrm{v}$. 


\subsection{Results}

From raw GNSS data (Figure 4.11), the slip offshore Gisborne is from 24th March to 1st May 2019, with the maximum displacement over $150 \mathrm{~mm}$ (Woods et al., 2020). The limitation here is that the timing is from the onshore GNSS data, and there is not yet a good constraint for the timing of the slip offshore.

The velocity variations at $1-2.5 \mathrm{~s}$ (Figure 4.12 ) show an approximate $0.02 \% \pm$ $0.008 \%$ decrease from the beginning of April to the beginning of May, during the Gisborne slip, followed by an increase after the SSE back to the average velocity, lasting about 15 days. A velocity increase before the SSE is observed at each individual station. These velocity increases are suppressed on the average result, possibly as a result of velocity increases occurring at different times at different stations.

A magnitude 5 earthquake is located about $50 \mathrm{~km}$ away from the deployment (Figure 4.1), occurring on 14th May 2019 (Figure 4.12). However, the velocities do not change after this earthquake, which is located far from the OBS deployment. There is no obvious velocity variation at $2.5-14 \mathrm{~s}$ band (Figure 4.13).

\subsection{Discussion}

Comparing to Chapter 3 which uses nine OBSs, this chapter uses fewer stations (five OBSs). The slip of the 2019 SSE (150 mm) under the deployment is also less than the 2014 SSE2 $(250 \mathrm{~mm})$ studied in Chapter 3. The velocity decrease during the 2019 SSE (on the order of $0.015 \%$ ) is less significant comparing to the velocity decrease (on the order of $0.05 \%$ ) during the 2014 SSE2 in Chapter 3.

\subsubsection{Depths of velocity variations}

Here we use the kernels computed in Chapter 3 to discuss the depth, because the velocity model and the period bands (2-20 s) are the same in Chapter 3 and this chapter. From the sensitivity kernels (Figures 3.3 and 3.4), 1-2.5 s surface waves are sensitive to very shallow depths (above $3 \mathrm{~km}$ ). While velocity variations with a bandpass of 1-2.5 s show changes during and after the 2019 Gisborne SSE, velocity variations with a bandpass of $2.5-14 \mathrm{~s}$, that sample deeper depths, do not 


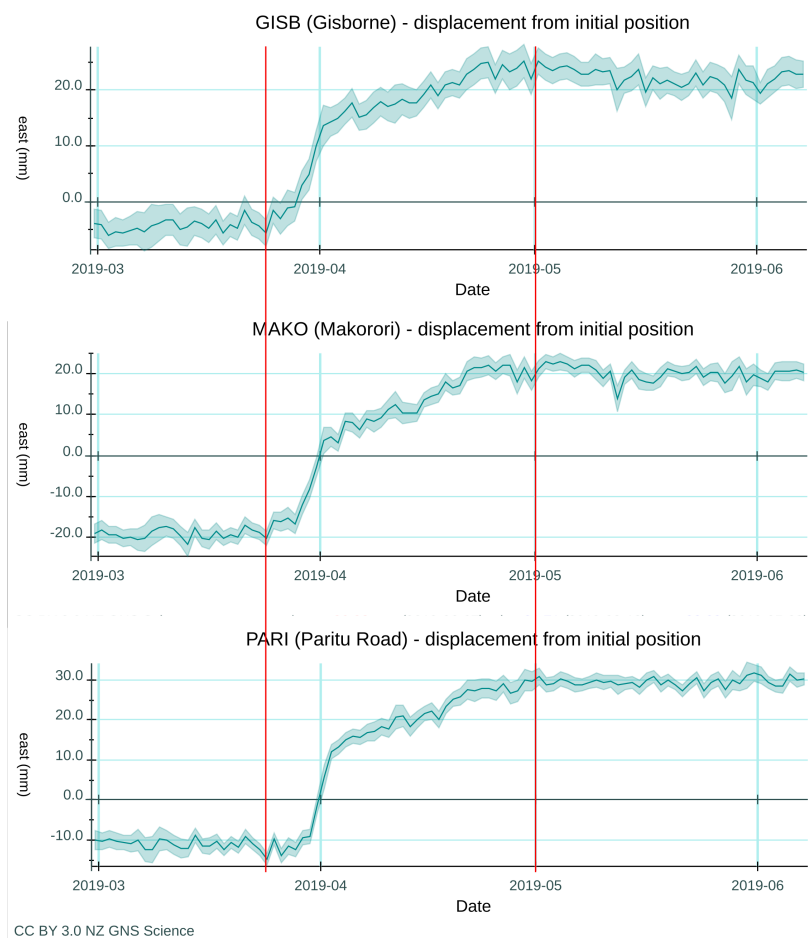

Figure 4.11: 2019 SSE timing from raw GNSS data. The locations of the three GNSS stations are shown in Figure 4.1. The slip offshore Gisborne is from 24th March to 1st May 2019, marked by the red lines.
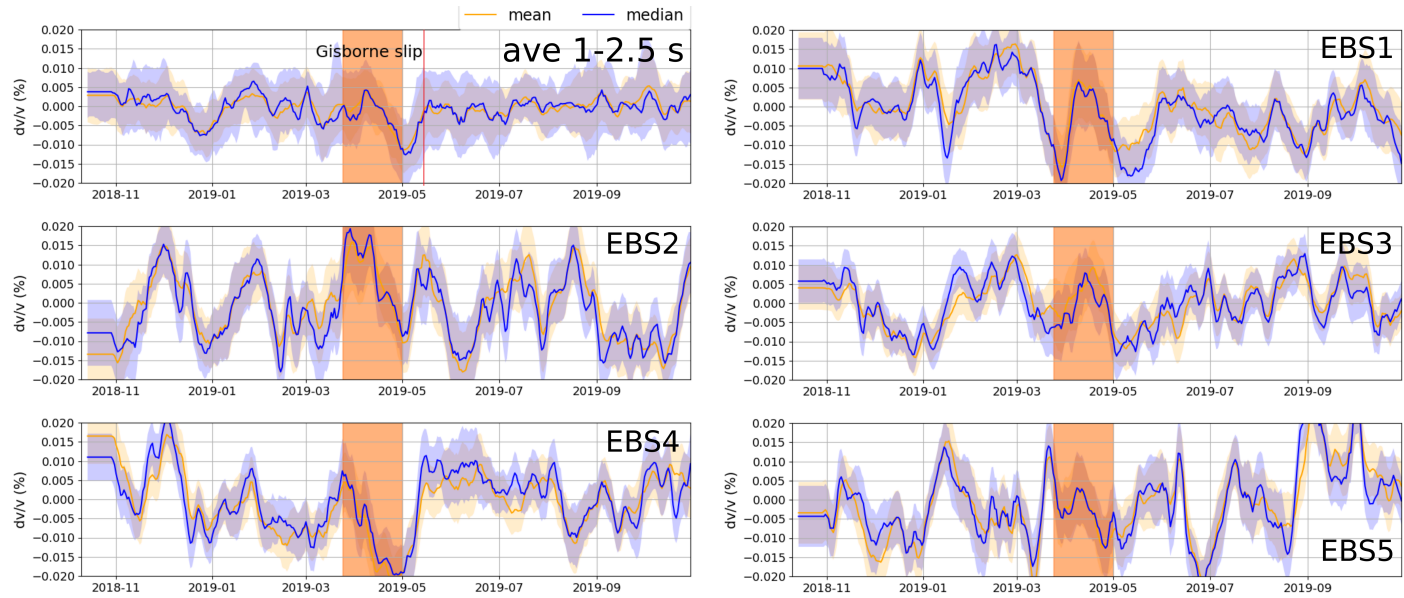

Figure 4.12: Velocity variations of single-station results and their average, at 1-2.5 s. Mean (orange) and median (blue) values of the five OBSs' velocity variations are shown with their uncertainties. The errors of the average $\mathrm{dv} / \mathrm{v}$ are calculated by a bootstrap method. The orange shades mark the slow slip event in 2019 offshore Gisborne. The red vertical line in the average result figure marks the magnitude 5 earthquake occurring on 14th May 2019 (Figure 4.1).

show obvious changes related to 2019 Gisborne SSE (Figure 4.13). In comparison, in Chapter 3, the velocity variations at 2.5-14 s show changes caused by the 2014 Gisborne SSE while the velocity variations at $1-2.5 \mathrm{~s}$ do not show changes (Figure 

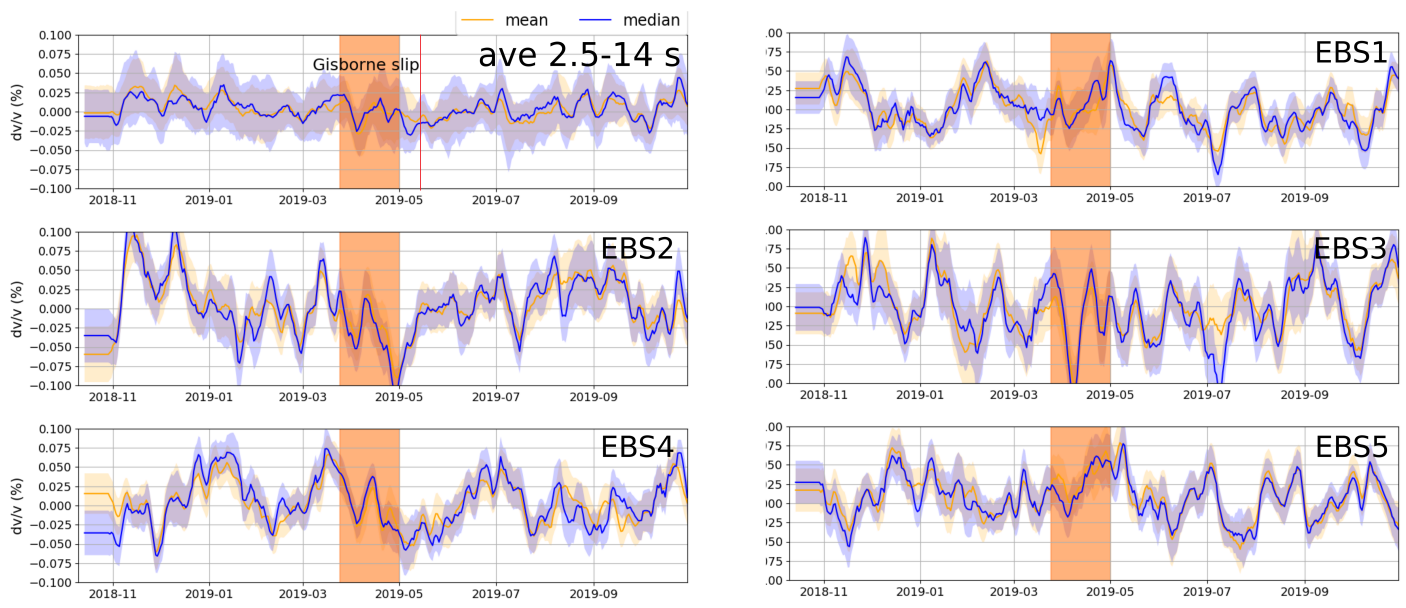

Figure 4.13: Velocity variations of single station results and their average, at 2.5-14 s.

A.11). Although from the first overtone kernels (Figure 3.4), $\sim 2 s$ is also sensitive to deeper depths in the upper plate, it is more sensitive to shallower depths. Comparing to the velocity variations caused by the 2014 Gisborne SSE (Chapter 3), the velocity variations caused by the 2019 Gisborne SSE (this chapter) sample shallower depths (mainly above $3 \mathrm{~km}$ ). Another possible reason why velocity variations are only observed at higher frequencies in this chapter is that this chapter only uses EOBSs, short-period instruments with a natural period of $1 \mathrm{~s}$. In contrast, broadband instruments (LOBSs) are included in Chapter 3. To test this, the velocity variations in 2014-2015 at 1-2.5 s and 2.5-14 s are computed using only EOBSs. Figure 4.14 shows that the velocities at 2.5-14 s have obvious changes while the velocity variations at $1-2.5 \mathrm{~s}$ are considerably smaller. Therefore, the reason that the velocity variations caused by the 2019 SSE are only at 1-2.5 s and the velocity variations caused by the 2014 SSE2 are only at 2.5-14 s is the depth difference rather than the effects of instruments.

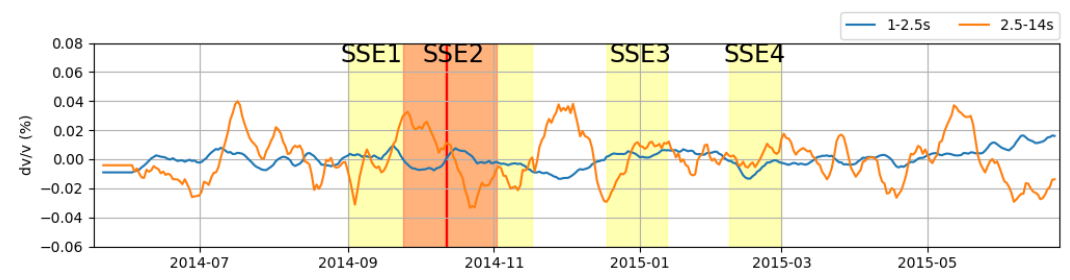

Figure 4.14: Velocity variations in 2014-2015 at 1-2.5 s and 2.5-14 s computed using only EOBSs. Refer to Chapter 3 for details.

In conclusion, the velocity variations during the 2014 SSE2 occurred at deeper depths, while the velocity variations during the 2019 SSE occurred at shallower 
depths.

\subsubsection{Velocity increases before the SSE}

All the single station results (1-2.5 s) show a velocity increase before the SSE, although it is not significant compared to the noise and this increase is not obvious in the average result. It may be possible that the SSE timing is different on different station locations and therefore the velocity increases before the SSE undergo destructive interference through averaging. The average velocity variations in 2014 (Figure 3.5 in Chapter 3) also show velocity increases before SSE2 and between SSE2 and SSE3. Both observations imply there might be a precursory signal - a velocity increase before an SSE. In Chapter 3, two hypotheses are proposed to interpret the velocity variations. 1) Fluid migration related to fault-valve behavior: the velocity decrease during an SSE is caused by more fluids migrating into the upper plate as the SSE breaks a low-permeability seal on the plate boundary. 2) Crustal strain changes through the SSE cycle: the velocity decrease during an SSE is caused by increased porosity because the SSE relieves the elastic strain, which results in dilation. A combination of both processes is also possible. Assuming the velocity increases before the SSEs are real, the second model can explain the observation better. Before an SSE, elastic strain accumulates, causing contraction and reduction of porosity, which results in a velocity increase.

If the velocity increases before the SSEs are real, since the fluid migration hypothesis cannot explain the velocity variations before the SSE, the interpretation can be improved as (Figure 4.15): Before the 2019 Gisborne SSE, the accumulated elastic strain compresses the upper plate materials causing a reduction of porosity, which leads to a seismic velocity increase. The occurrence of the SSE releases the accumulated strain. It causes a dilation and porosity increase, which results in a seismic velocity reduction. Accompanied by a porosity increase during the SSE, there might be a fluid volume increase, and fluids may fill into the increased pores. The fluids may come from the interface or lower plate as a result of the SSE breaking the low permeability seal on the interface and the fluids becoming interconnected (Warren-Smith et al., 2019; Zal et al., 2020). After the SSE, the elastic strain starts to accumulate again, causing a contraction and reduction of porosity, which results in a velocity increase that comes back to the average velocity. Meanwhile, the plate boundary re-seals, and the fluids in the upper plate diffuse. 
Using the same process to interpret the velocity variations in 2014-2015 (Figures 3.7), the velocity increase before SSE2 might be caused by porosity reduction because of the accumulated elastic strain. The velocity increase is less likely to be related to SSE1 because SSE1 did not happen within the deployment region (Figure A.2). During SSE2, the accumulated strain is released, resulting in a dilation and porosity increase. An increased porosity results in a velocity decrease. At the same time, fluids around the interface may migrate to the upper plate and fill the increased pores. After SSE2, elastic strain accumulates again prior to SSE3, causing contraction, porosity reduction, and increasing velocity. It is also possible that the increased strain before SSE3 squeezes the pores and causes an increased pore fluid pressure or decreased fluid volume if the fluids diffuse, causing a velocity increase. It is more likely that the strain variations are dominant in velocity variations because the fluid migration hypothesis cannot explain the velocity changes before the SSEs. With only the influence from fluid diffusion, the velocity increase after SSE2 cannot be over the original velocity values, although it remains unclear if it is over the original velocity values considering the errors. The same process may happen to SSE3.

From the timing of the velocity variations at $1-2.5 \mathrm{~s}$ on different single stations (Figure 4.12), it is plausible that the SSE started from the north (EBS5, EBS4, EBS2) and migrated to the south (EBS1, EBS3). Because of the time shift, the velocity increases before the SSE shown on the single station results may have undergone destructive interference through averaging the single station velocity variations, becoming obscured in the averaged result. The timing difference is within two weeks, which requires a high temporal resolution with geodesy. It is difficult to be constrained given that the seafloor geodetic instruments (pink circles in Figure 4.16) only cover the southern part of the OBS network.

\subsubsection{Porosity changes}

Similar to Chapter 3, porosity and volume changes are estimated and compared. The velocity decrease caused by the 2014 Gisborne SSE (Chapter 3) is $0.06 \% \pm$ $0.03 \%$, and the velocity decrease caused by the 2019 Gisborne SSE (this chapter) is $0.02 \% \pm 0.008 \%$. Following the same analysis, the effective porosity increase during the 2019 Gisborne SSE is estimated to be the same order, $0.0001 \%$, as during the 2014 SSE2. The percentage change of porosity is estimated to be the same 


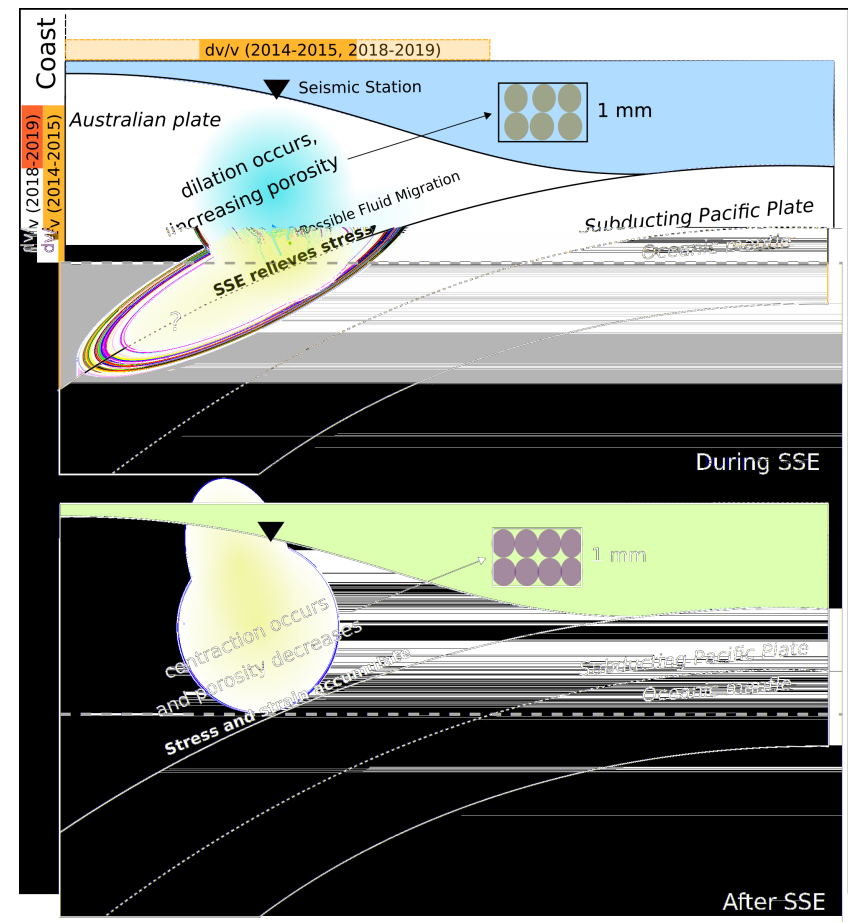

Figure 4.15: Interpretation schematic model adapted after Husen and Kissling (2001), Zal et al. (2020), and Chapter 3. The horizontal grey dashed lines mark the depths of the plate interface under the seismic station, referring to the sensitivity kernels (Figure 3.3). The bars on the top and left of the figure show the horizontal and vertical space coverage of the $\mathrm{dv} / \mathrm{v}$ observations on 20142015 and 2018-2019. The main observation is considered to be under the network (orange), though it may extend over a wider region (light orange). The horizontal coverage of the $\mathrm{dv} / \mathrm{v}$ in 2014-2015 and 2018-2019 is similar; both are around the OBS deployments. The vertical coverage of the two observations is different.

in both years, from $0.0003 \%$ to $0.0005 \%$. For the volume change estimation, the only parameter different from Chapter 3 is that the amount of slip is $0.15 \mathrm{~m}$. The stress drop $\delta \sigma_{\text {static }}=G d / w$ (Stein and Wysession, 2009) during the 2019 Gisborne SSE is estimated to be $18 \mathrm{kPa}$. Then the percent volume change is estimated to be $0.00025 \%$, which is also in accordance with the porosity change assuming the porosity change is caused by the volume change. However, even though the velocity change during the 2019 Gisborne SSE $(0.02 \%)$ is obviously different from the velocity change during the 2014 Gisborne SSE $(0.06 \%)$, the porosity changes estimated from these two velocity changes are still on the same order of magnitude. This suggests that estimating porosity changes from velocity changes cannot provide a good resolution, and seismic velocity variations may not monitor minute strains in the hanging wall of subduction zone megathrusts based on the current methodology. In this study, porosity is estimated from velocity considering lab 


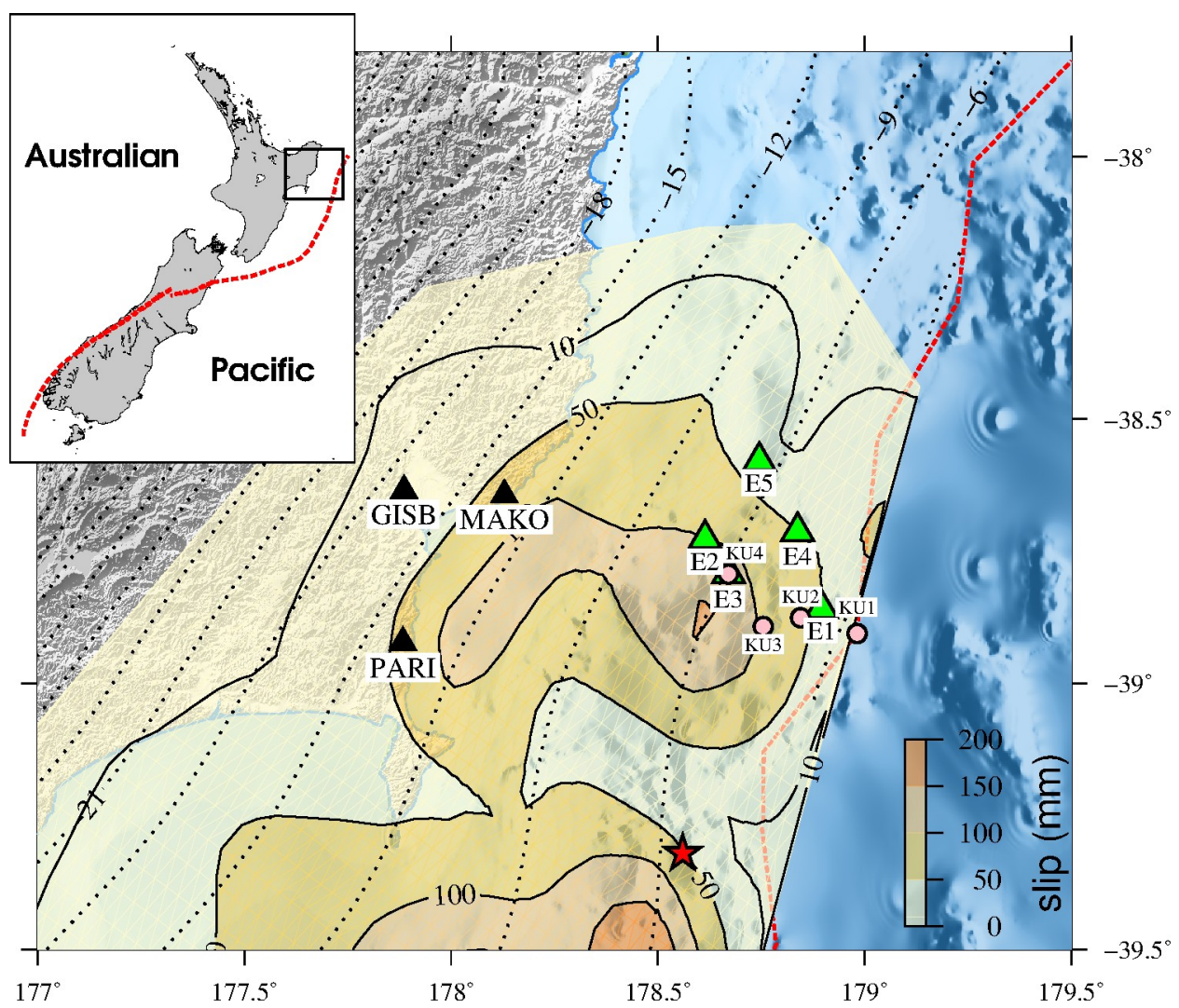

Figure 4.16: Locations of bottom pressure recorders 2018-2019, plotted based on Figure 4.1. The pink circles denote the bottom pressure recorders.

data from experiments on particular rock types (poorly consolidated sandstones, limestones, or mudstones; Mavko et al., 2020; Hoffman and Tobin, 2002). However, the real underground texture is more complicated. Even though we have information from drilling (Wallace et al., 2019), it is hard to identify a precise relationship between porosity and velocity, and therefore we can only estimate an order of porosity magnitude.

\subsection{Conclusions}

We analyse one-year ambient noise data acquired by five OBSs deployed from October of 2018 to October of 2019 in the northern Hikurangi Margin in a region where an SSE occurred from the end of March to the beginning of May, lasting about five weeks. Temporal velocity variations are computed using coda waves retrieved from single station cross-component correlations. The average velocity variations exhibit a velocity decrease during the SSE. The velocity decrease is followed by an increase after the SSE, returning to original values. While it is not 
obvious on the average velocity variations, the single station velocity variations exhibit a velocity increase before the SSE. While the velocity decrease during the SSE and increase after SSE support both hypotheses (strain changes and fluid migration), the velocity increase before the SSE tend to support the strain change hypothesis.

This study provides more evidence to support that velocity variations associated with the occurrence of SSEs in New Zealand are detectable using ambient noise interferometry, and results provide evidence to support the integral role that accumulated elastic strain energy and/or fluids play in promoting slow slip events on the northern Hikurangi Margin.

We have found that:

1. The depth of velocity variations caused by the 2019 SSE is shallower than the velocity variations caused by the 2014 SSE2.

2. There is likely a precursor signal of velocity increase before SSEs, considering the velocity increases before the SSEs in Chapter 3 and assuming the velocity increases before the 2019 SSE observed in single stations in this chapter are real.

3. The hypothesis of crustal strain changes through the SSE cycle can better explain the velocity increase before SSEs than the hypothesis of fluid migration related to fault-valve behavior. 
4. Temporal velocity variations in 2018-2019 in the northern Hikurangi margin and the relation to slow slip 


\section{Chapter 5}

\section{Studies of shear wave velocity structure in the southwestern Okinawa Trough from continuous OBS data}

\subsection{Abstract}

The southwestern Okinawa Trough is an active back-arc basin, extending and rifting within the continental lithosphere. Studying the southwestern Okinawa Trough is important to understand the opening and development of a back-arc basin. At various times between 2010 and 2018, 34 Ocean Bottom Seismometers (OBSs) on a small scale $\left(\sim 0.2^{\circ} \times 0.3^{\circ}\right)$ were deployed by Academia Sinica in southwestern Okinawa Trough offshore northeast Taiwan. Ambient noise recorded on vertical velocity and pressure sensors is used to retrieve Rayleigh/Scholte waves to study the shear wave velocity structure. A phase velocity dispersion curve is forward-modeled, according to a shear velocity model from an adjacent region proposed by Kuo et al. (2015), and to shear strength and density data from Ocean Drilling Program (ODP) Site 1202. Phase velocity dispersion curves are measured from the retrieved Rayleigh/Scholte waves and unwrapped according to which branch of the modeled phase velocities they appear closest to. The phase velocities measured from the stations in the north of the rifting axis $(714 \pm 39 \mathrm{~m} / \mathrm{s}$ at 3.5 
$\mathrm{s}$ and $1305 \pm 145 \mathrm{~m} / \mathrm{s}$ at $5.8 \mathrm{~s}$ ) are higher than the phase velocities measured from the stations in the south $(632 \pm 22 \mathrm{~m} / \mathrm{s}$ at $3.5 \mathrm{~s}$ and $\sim 1100 \mathrm{~m} / \mathrm{s}$ at $5.8 \mathrm{~s})$. Shear wave velocities are inverted using the phase velocity dispersion curves, suggesting the shear velocity in the south is slower than that in the north of the rifting axis. Previous studies have shown high heat flows (about $110 \mathrm{~mW} / \mathrm{m}^{2}$ on average) in the south of the rifting axis. The low velocity in the south can be caused by the high heat flow in the south region, which may be a consequence of asymmetric backarc extension and/or rifting. The asymmetric (along the rifting axis) shear wave velocities observed in this study may imply that the back-arc extending/rifting is asymmetric in the study region.

\subsection{Study region and tectonic background}

Taiwan is formed by the orogeny from the collision of the Eurasian Plate and the Philippine Sea Plate (Figure 1.4; Figure 5.1), which forms a plate-convergent margin (Sibuet and Hsu, 2004). The plate-convergent margin in Taiwan is composed of two subduction zones of opposite polarities (Wu et al., 2007), which is similar to the tectonic setting of New Zealand. In east offshore Taiwan, the Philippine Sea Plate subducts under the Eurasian Plate along Ryukyu Trench, forming the Ryukyu Arc and Okinawa Trough, while in south offshore Taiwan, the Eurasian Plate subducts under Philippine Sea Plate along Manila Trench, which forms Luzon Arc (Ramsey et al., 2006).

In offshore eastern Taiwan, the Philippine Sea Plate is subducting under the Ryukyu Trench at a rate of about $82 \mathrm{~mm} / \mathrm{yr}$ (Seno et al., 1993). At the same time, the Ryukyu arc retreats southwards at a rate of about $70 \mathrm{~mm} / \mathrm{yr}$ because of the oblique subduction (Figure 5.1). Long-term seafloor geodetic measurements near the axis of the Okinawa Trough back-arc basin from 2009 to 2019 (Chen et al., 2018, 2021) suggested the tectonics in the southwestern Okinawa Trough are complicated (Figure 5.1).

The Okinawa Trough is a curved back-arc basin where the most notable tectonic processes are the active extension and rifting within the continental lithosphere. Continental rifting along the Okinawa Trough has occurred in three phases since initiation (Sibuet et al., 1995). The first phase of rifting started in the Middle Miocene (12 Ma) or Late Miocene (6 Ma), with the most significant extension of 
50 to $75 \mathrm{~km}$. The second and third rifting phases occurred in the Late PliocenePleistocene and Late Pleistocene to Recent, involving an extension of 25 to $30 \mathrm{~km}$ in total. Rifting within the continental lithosphere is an important tectonic activity for lithospheric evolution in an extensional stress condition. It can widen the back-arc basin and accumulate more sediment or lead to the breaking of continental lithosphere (Kearey et al., 2009). The continental lithosphere of the central and southern Okinawa Trough has ruptured along the rifting axis (Figure 5.2), and the new oceanic crust is developing at the rifting center since 2 Ma (Liu et al., 2016). The back-arc extension is believed to have started recently (upper Pleistocene) in an N-S direction (Sibuet et al., 1998; Shyu and Liu, 2001). The rifting and extension of the Okinawa Trough enhance the Philippine Sea Plate subduction rates. By comparing with global passive continental margins and marginal basins in the West Pacific, it is suggested that the central and southern Okinawa Trough is at the early stage of seafloor spreading, generating new oceanic crust at the spreading center, while the northern Okinawa Trough is at the mature stage of continental rifting (Liu et al., 2016). Previous studies on the helium isotope of volcanic rocks indicated that the southern Okinawa Trough spreads faster than the central Okinawa Trough (Yu et al., 2016).

The thickness of the crust in the northern Okinawa Trough is $30 \mathrm{~km}$, thinning to $10 \mathrm{~km}$ in the southern Okinawa Trough (Taylor, 2013). In the study region of this chapter, the crust thickness is about $25 \mathrm{~km}$ (Han et al., 2007; Jia and Sun, 2021) and the depth to the top of the slab is around $125 \mathrm{~km}$ (Chou et al., 2006). Sibuet et al. (1998) suggested a region with a cross-back-arc volcanic trail (Figure 5.2), which was considered as voluminous anomalous volcanism. This is located beside our study region (Figure 5.2). Chung et al. (2000) suggested that these volcanoes were the products of arc magmatism. It is common to have many volcanoes where the slab is about $100 \mathrm{~km}$ deep (Syracuse and Abers, 2006).

Previous geothermal studies since the 1960s have revealed that heat flow is generally high in back-arc basins (e.g., Watanabe et al., 1970; Yasui et al., 2012). From studies on the relationship between heat flow and the age of marginal basins (Sclater et al., 1980; Yamano, 1988), it seems that the relationship between heat flow and basin age is similar for ocean basins, suggesting that the thermal evolution of marginal basin lithosphere is similar to that of oceanic lithosphere. Heat flow measurements (about $110 \mathrm{~mW} / \mathrm{m}^{2}$ on average, varying from 9 to $437 \mathrm{~mW} / \mathrm{m}^{2}$; 
Shyu and Liu, 2001) in the southwestern Okinawa Trough are high and are related to continental rifting and seafloor spreading (Liu et al., 2016; Chen et al., 2020). High heat flows (Yamano et al., 1989; Kinoshita and Yamano, 1997; Shyu and Liu, 2001) indicate continuous spreading and volcanism in the mid and southern Okinawa Trough. East of my study region, there is a spot (the red circle in Figure 5.2) with particularly high heat flow $\left(3000 \mathrm{~mW} / \mathrm{m}^{2}\right)$ compared to the surroundings (Yamano et al., 1989; Shyu and Liu, 2001; Gutscher et al., 2016; Liu et al., 2016; Wu et al., 2019; Chen et al., 2020). Chen et al. (2020) suggested there is a magma chamber beneath it and the hydrothermal fluid here might mix with the magmatic body. The magmatism is intense in the rift center, and linear magnetic stripes are identified in the southern Okinawa Trough (Kimura, 1985; Sibuet et al., 1987; Liang et al., 2002; Liu et al., 2016). Crustal magmatic bodies are generally characterized by low Vp, low Vs, and high Vp/Vs (Ito et al., 1979; Mavko and Mukerji, 1995). Lin et al. (2007) studied Vp/Vs (Figure 5.2) using earthquake tomography in the southern Okinawa Trough and found most earthquakes in the southwestern Okinawa Trough were located around low Vp and Vs, and high Vp/Vs bodies, suggesting the seismicity was related to magmatic and/or fluid activities.

In contrast to normal mid-ocean ridges, back-arc basins can be characterized by asymmetric seafloor spreading, which means one side is spreading and the other side dose not. This asymmetry is variable for different basins or even within single basins (Deschamps and Fujiwara, 2003; Martinez et al., 2000). The cause of asymmetric spreading in back-arc basins remains poorly understood. Some hypotheses invoke asymmetries relative to the spreading axis in arc melt generation processes and heat flow, hydration gradients with distance from the slab, mantle wedge effects, and evolution from rifting to spreading (Barker and Hill, 1980; Martínez et al., 1995; Molnar and Atwater, 1978). In some cases, volcanic activities are asymmetric relative to the extensional axes and occasionally exhibit alongaxis variations in eruption loci. Examples are the Taupo Volcanic Zone (Stern, 1987; Hamling et al., 2016) and the Ethiopian Rift (Keir et al., 2015). Stern (1987) and Stern and Benson (2011) studied the Taupo Volcanic Zone and suggested the asymmetric distribution of heat output may be caused by the asymmetric back-arc spreading. Southeast rollback of the subduction zone leads to thinned lithosphere in the east of the Taupo Volcanic Zone and a higher heat output than the west.

The Kuroshio (Black) Current is the biggest western boundary surface current in 
the western Pacific. To study the Kuroshio (Black) Current, Ocean Drilling Program (ODP) Site 1202 was drilled at $24^{\circ} 48.24^{\prime} N, 122^{\circ} 30.00^{\prime} E$ (Salisbury, 2002), which is within the study region of this chapter (Figure 5.2). Four holes were drilled, providing peak shear strength down to 120 meters below sea floor, $\mathrm{Vp}$ down to 15 meters below sea floor, and density down to 140 meters below sea floor.

This study uses seismic ambient noise data collected at the southwestern edge of the Okinawa Trough, where the rifting terminates, to study the shear velocity structure and the back-arc tectonics of this region.

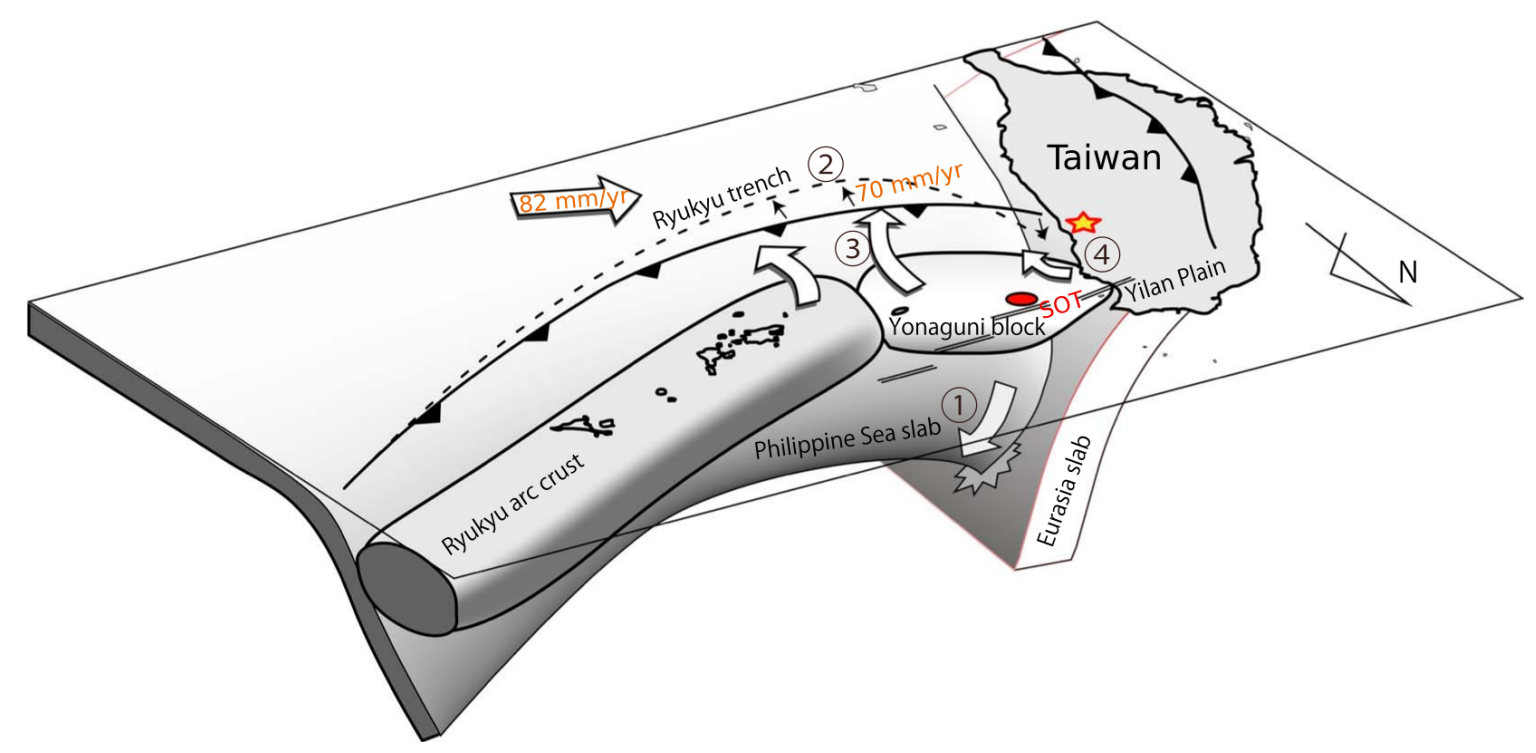

Figure 5.1: Illustration of tectonics northeastern offshore Taiwan suggested by GNSS studies (Chen et al., 2018, 2021). The location of this study is on the red circle (SOT: Southwestern Okinawa Trough). The Philippine Sea Plate is subducting under the Ryukyu Trench at a rate of about 82 $\mathrm{mm} / \mathrm{yr}$ (Seno et al., 1993) while the Ryukyu arc retreats southwards at a rate of about $70 \mathrm{~mm} / \mathrm{yr}$ (Nakamura, 2004). Open arrows represent the motion of the slab and blocks. The broken line shows the deformation of the Ryukyu trench. (1) the Philippine Sea slab slides down along the slope of the Eurasia slab. (2) the Ryukyu trench retreats southward. (3) the slab suction makes Ryukyu arc and the Yonaguni block rotate. (4) the Yonaguni block pulls the Yilan Plain southeastward. [From Chen et al. (2018)] 


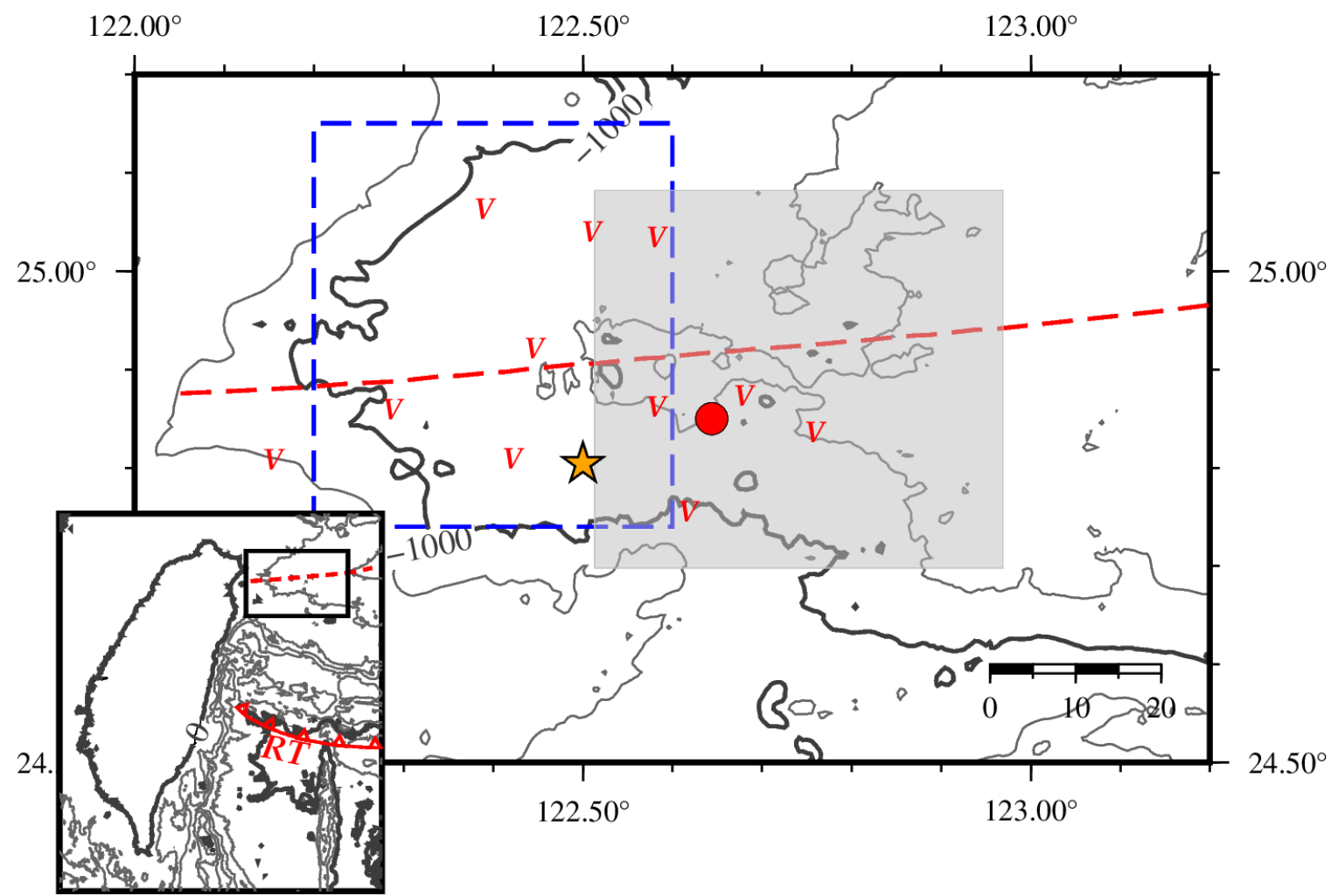

Figure 5.2: Hot vents in southwestern Okinawa Trough. The blue dashed rectangle outlines the study region in this chapter. The grey shade indicates a cross-back-arc volcanic trail (Sibuet et al., 1998). In the same region, Lin et al. (2007) suggested high Vp/Vs values (>1.78) and low Vp and Vs values where most earthquakes were located. Red $\mathrm{V}$ mark the locations of detected hot vents (Lee, 2005). The red circle denotes a spot with particularly high heat flow $\left(3000 \mathrm{~mW} / \mathrm{m}^{2}\right.$; Chen et al., 2020). The red dashed line denotes the rifting axis of Okinawa Trough (Sibuet et al., 1998). The orange star denotes the position of ODP1202 (Salisbury, 2002). 


\subsection{Data}

From 2010 to 2018, the Institute of Earth Sciences (IES), Taiwan deployed 34 OBSs during different times (Figure 5.3) in southwestern Okinawa Trough. The OBSs were of two types: one is called Lobster from Germany, and the other one is called Yarbird from IES. Yarbird instruments were produced by IES and have been frequently updated since 2010. We name different types of Yarbird instruments according to the low corner frequency of the instrument response, e.g., if the low corner frequency is $3 \mathrm{~s}$, the instrument is called Yarbird-3s. Among the 34 OBSs, 5 of them are only equipped with 3-component seismometers, and 29 of them are equipped with differential pressure gauges and 3-component seismometers.

Table 5.1 shows the information about the deployment and different types of instruments, and Figure 5.4 shows the corresponding instrument responses. As all the instruments in the southwestern Okinawa Trough use the same differential pressure gauge type, instrument responses of differential pressure gauges are not considered during data processing. Instrument responses of seismic vertical components are only removed if the instruments deployed during the same time are different types.

During the processing, the first overview of the data quality is a power spectral density plot. An example power spectral density from this study (Figure 5.5) shows the data are noisier than the global average high-noise model at most periods. 


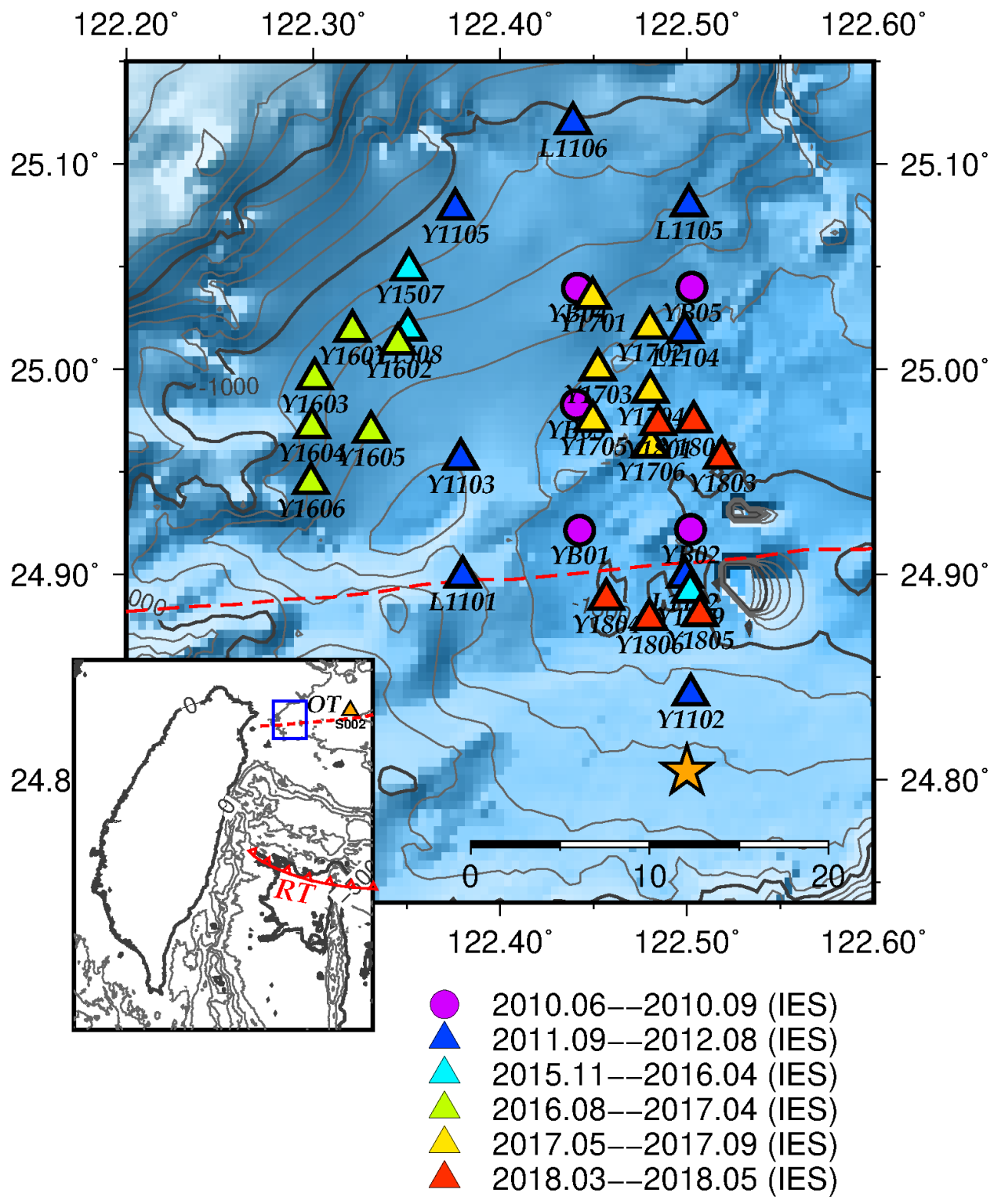

Figure 5.3: Deployment of Ocean Bottom Seismometers (OBSs) in the southwestern Okinawa Trough. 34 OBSs were deployed at different times, denoted by different colors. Circles indicate the instruments equipped with 3-component seismometers only; triangles indicate the instruments equipped with differential pressure gauges and 3-component seismometers. $\mathrm{L}$ is for Lobster and $\mathrm{Y}$ is for Yarbird. The red dashed line denotes the rifting axis of Okinawa Trough (Sibuet et al., 1998). The orange star shows the position of ODP1202 (Salisbury, 2002). The blue rectangle in the inset denotes the deployment region. The orange triangle denotes a station $\mathrm{S} 002$ used in a previous study (Kuo et al., 2015). A Vs model on site $S 002$ was proposed (Kuo et al., 2015), which is used as a reference model in this chapter. EP, Eurasian Plate; PSP, Philippine Sea Plate; OT, Okinawa Trough; RT, Ryukyu Trench. 
Table 5.1: Deployment and instrumentation information. Yarbird uses the same type of differential pressure gauge as Lobster. The max number of cross-correlations (CC) is computed by permutation $C_{n}^{2}$, where $n$ is the number of stations during the same time period, 2 stands for 'every 2 stations compute one CC'. CC: cross-correlations; BB: Broadband.

\begin{tabular}{lccc}
\hline Time & Number of stations & Max number of CC & Instrument type \\
\hline $2010.06-2010.09$ & 5 & 10 & Yarbird-3s \\
$2011.09-2012.08$ & 8 & 28 & 3 Yarbird-7s, 5 Lobster \\
$2015.11-2016.04$ & 3 & 3 & Yarbird-BB \\
$2016.08-2017.04$ & 6 & 15 & Yarbird-BB \\
$2017.06-2017.10$ & 6 & 15 & Yarbird-BB \\
$2018.04-2018.06$ & 6 & 15 & Yarbird-BB \\
In total & 34 & 86 & \\
\hline
\end{tabular}

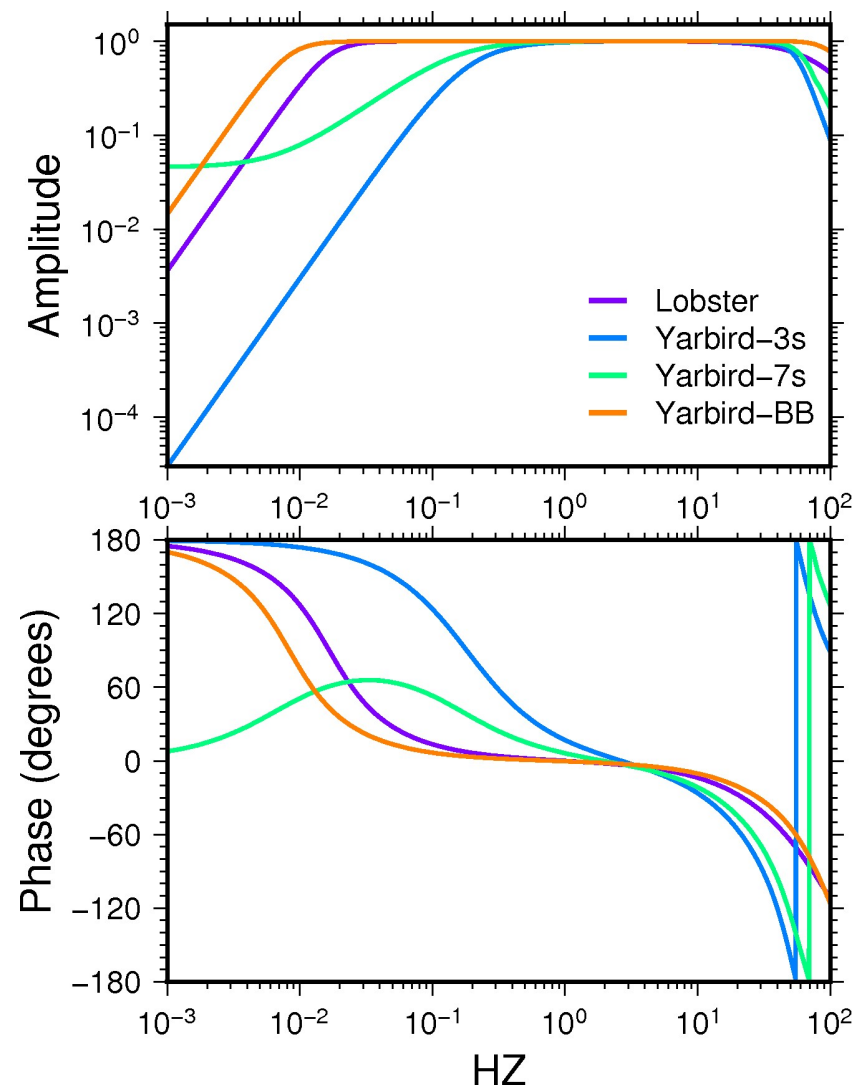

Figure 5.4: Velocity responses of the vertical components of different instruments deployed in southwestern Okinawa Trough with amplitude normalized to 1, corresponding to the instrument types in Table 5.1. 


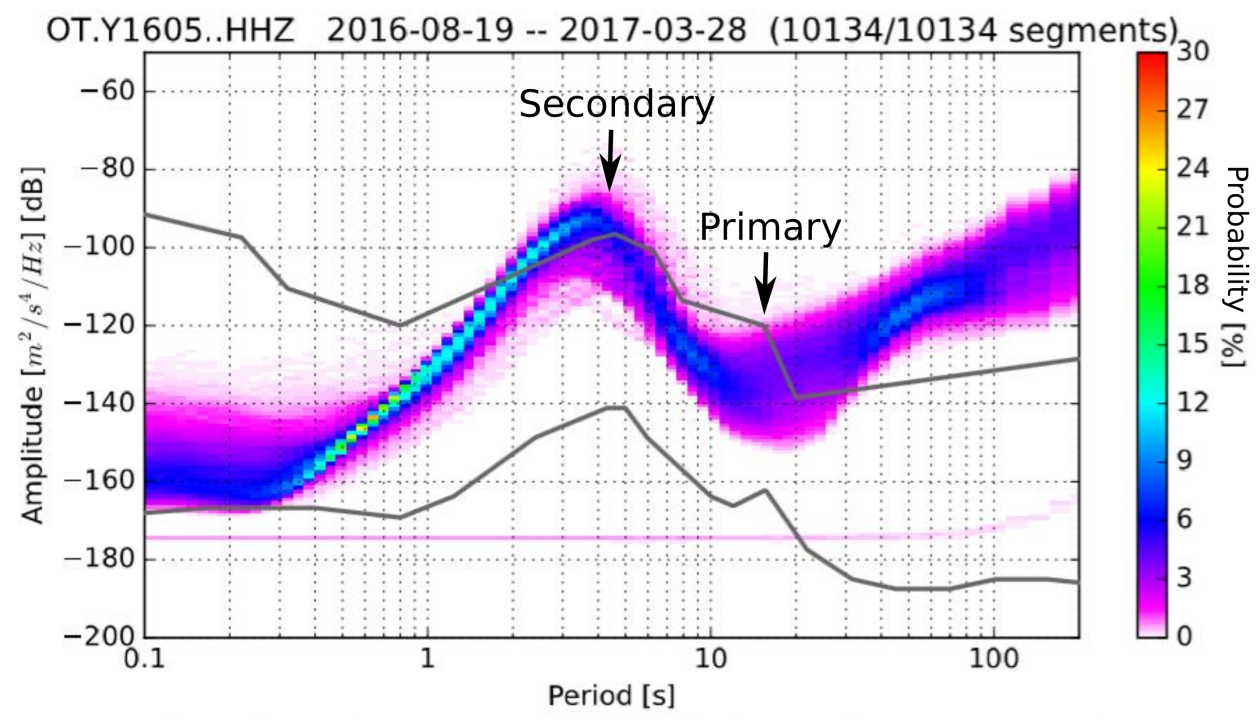

Figure 5.5: Power spectral density of ambient noise recorded at Y1605. The worldwide high and low noise levels from land stations (Peterson et al., 1993) are shown by grey curves for comparison. The two arrows mark the peaks of primary microseism and secondary microseism.

\subsection{Method}

\subsubsection{Ambient noise cross-correlations}

Station pair cross-correlations are computed on both differential pressure gauges and seismic vertical continuous data. In this study, cross-correlation functions are computed using MSnoise 1.5 (Lecocq et al., 2014).

\section{Parameterization}

Parameters in MSnoise are tested before processing data. When testing one particular parameter, other parameters are fixed. Some parameters do not affect the results, but two parameters, including time-domain normalization and stack method, strongly influence the cross-correlation computation in this study.

For time-domain normalization, the user can clip amplitudes to an integer value of the Root-Mean-Square of the trace or apply one-bit normalization that sets all positive values to be 1 and negative values to be -1 . Both ways can flatten the amplitude in the time domain. The comparison of Root-Mean-Square and one-bit normalization (Figure 5.6) shows that for long interstation distance, Root-MeanSquare normalization gives a clearer waveform than 1-bit; for short interstation 
distance, 1-bit normalization gives a better result than Root-Mean-Square. For short interstation distance, as the signals in positive lag and negative lag are close to each other, the zero-point noise caused by earthquakes mixes with the signals. 1-bit normalization works better for suppressing earthquakes. Therefore, RootMean-Square is used for long interstation distance pairs ( $>5 \mathrm{~km})$, and 1-bit normalization is used for short interstation distance pairs $(<5 \mathrm{~km})$. Different times of Root-Mean-Square do not make much difference for cross-correlation computation (Figure 5.7).

Choices of the stacking methods are linear stacking or phase-weighted stacking (Schimmel and Paulssen, 1997). Phase-weighted stacking can result in clearer waveforms (Figure 5.8). To make sure the phase-weighted stacking does not bias original phases, phase velocities of phase-weighted stacking and linear stacking are compared (Figure 5.8). Phase velocity measurements show that the phaseweighted stacking does not change the phase because the phase velocities measured from phase-weighted stack and linear stack are the same. Phase-weighted stacking is then conducted because it results in clearer cross-correlation waveforms.

After daily cross-correlation functions are computed, data are selected by calculating the correlation between the all-day-stacked cross-correlation function and single daily cross-correlation functions. If the correlation is larger than a threshold, one daily cross-correlation is used for the stack. Parameter thresholds of 0.1 , $0.2,0.3,0.4,0.5$ are tested. Examples of threshold $=0.1$ and 0.4 are shown in Figure 5.9. The stacked cross-correlation functions after selecting data have clearer signals (less noise) than the all-stacked cross-correlation function. But the positive signal (at $\sim 30 \mathrm{~s}$ lag time) is too weak if a high threshold is chosen, and the threshold of 0.1 is enough to improve the signal and suppress the noise. Therefore, 0.1 is chosen as the threshold to select data. That is, if the correlation between a daily cross-correlation function and the all-stacked cross-correlation function is bigger than 0.1 , the daily cross-correlation function would be used for stacking. 

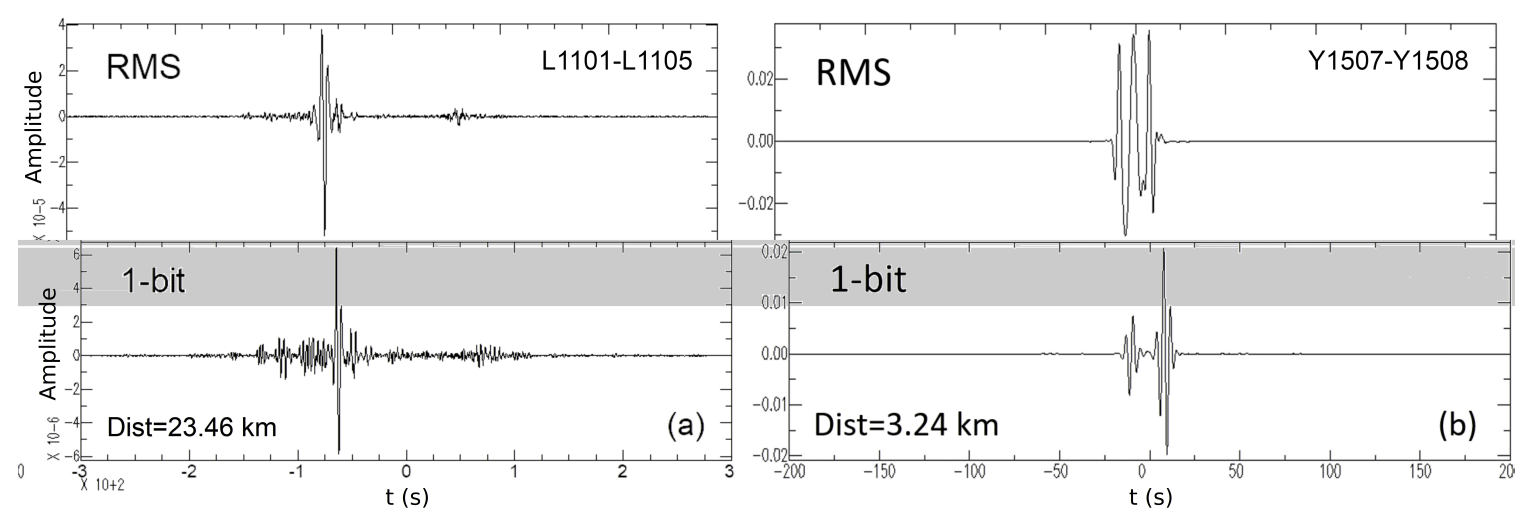

Figure 5.6: Comparison of three times Root-Mean-Square (RMS) and 1-bit normalization. Phase weighted stacking is used. (a) Comparison on station pair L1101-L1105 with long interstation distance $(23.46 \mathrm{~km})$. The cross-correlation processed by Root-Mean-Square normalization has a clearer signal; (b) Comparison on station pair Y1507-Y1508 with short interstation distance (3.24 $\mathrm{km})$. The cross-correlation processed by 1-bit has less zero-point noise. 

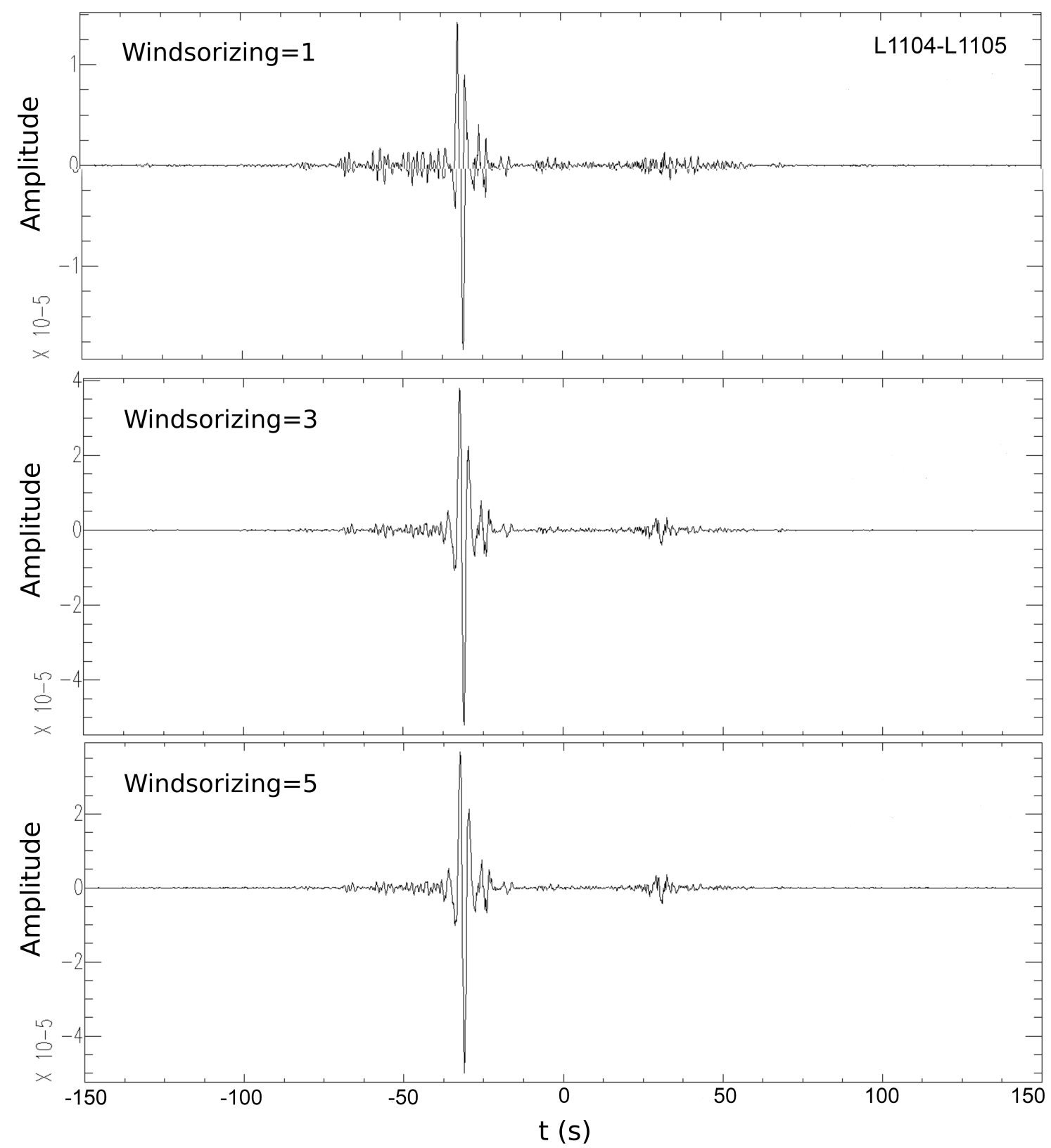

Figure 5.7: Comparison of one times, three times, and five times Root-Mean-Square on station pair L1104-L1105. Phase weighted stacking is used. Cross-correlations computed using (a) one, (b) three, and (c) five times Root-Mean-Square. 

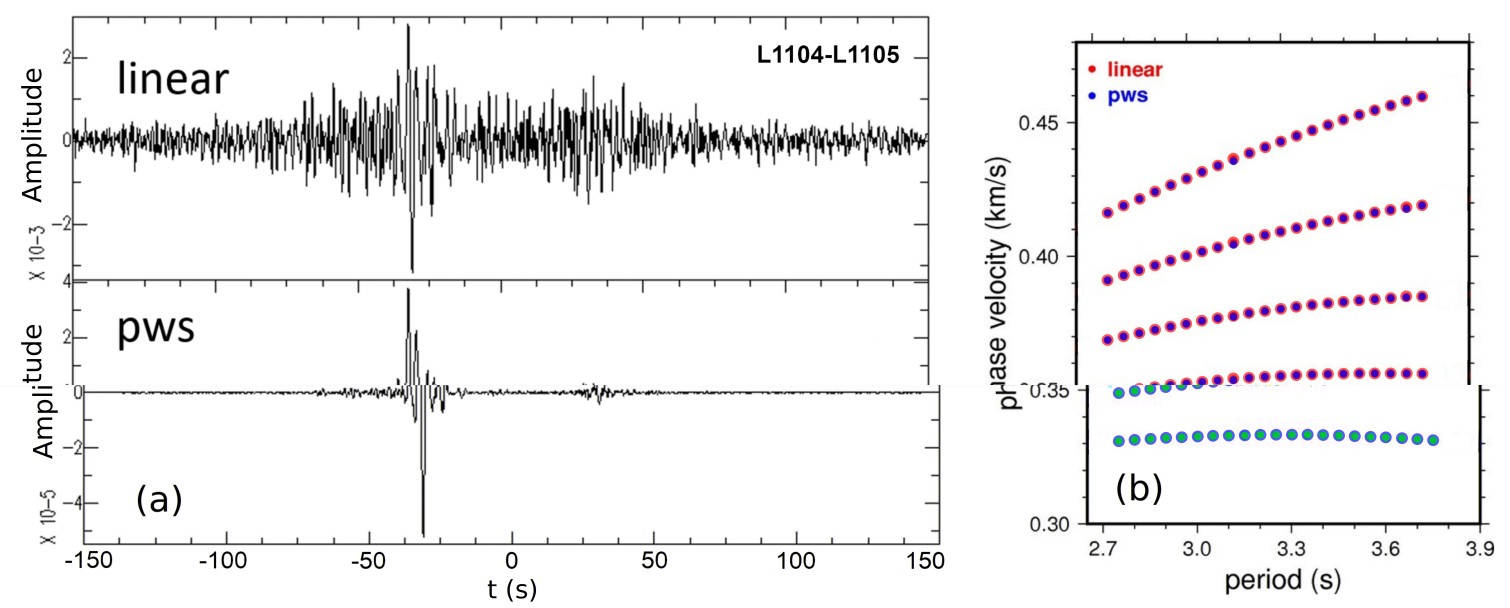

Figure 5.8: Comparison of the phase information of linear stack and phase-weighted stack on station pair L1104-L1105. (a) Comparison of the linear stack (top) and phase-weighted stack (bottom). (b) Comparison of the phase velocities measured from the linear stack and phase-weighted stack. The two measurements overlap with each other.

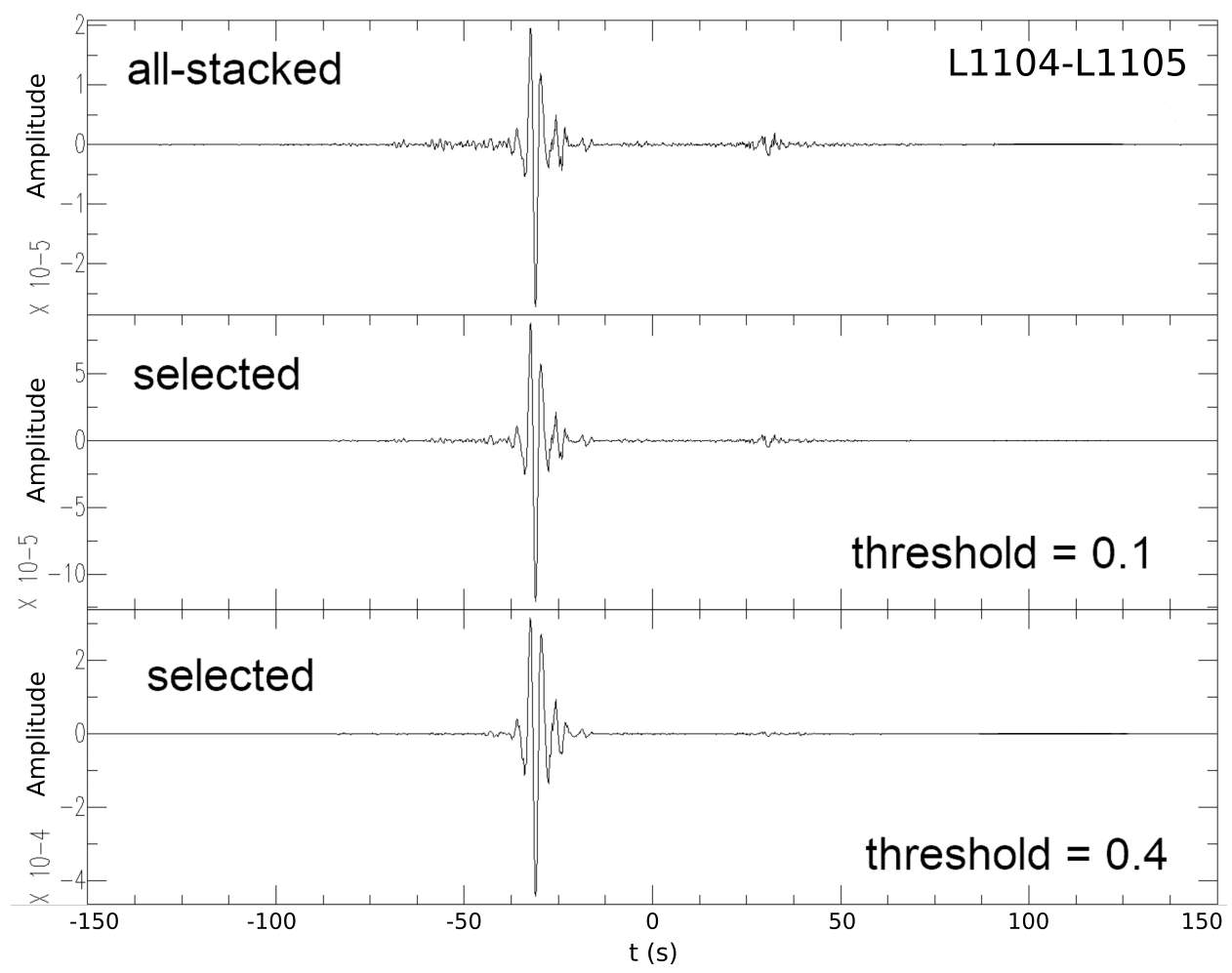

Figure 5.9: Comparison of the stacking using all days, and the stacking using the daily crosscorrelations selected by the correlation between all-day stack and daily cross-correlations bigger than 0.1 and 0.4 , respectively, on station pair L1104-L1105. 


\section{Cross-correlation Functions}

Figure 5.10 shows the cross-correlations derived from pressure gauges and seismic vertical components. The arrivals are considered to be Rayleigh/Scholte waves. Rayleigh waves including both radial and vertical motions are surface waves propagating on a solid-solid interface, while Scholte waves have the same physical properties but propagates on a fluid-solid interface. The cross-correlation functions derived from seismic vertical components and pressure gauges show similar fundamental mode arrivals. Therefore, for one station pair, the cross-correlation function with clearer signals is used. For the station pairs without pressure gauges equipped, the cross-correlations derived from the seismic vertical components are used. The earlier arrivals show a velocity close to the sound wave speed in the water, and they are mostly only observed by pressure sensors. We prove in Section 5.4.2 that the earlier arrivals couple well with the solid earth instead of just traveling in seawater by correction of compliance and tilt noise.

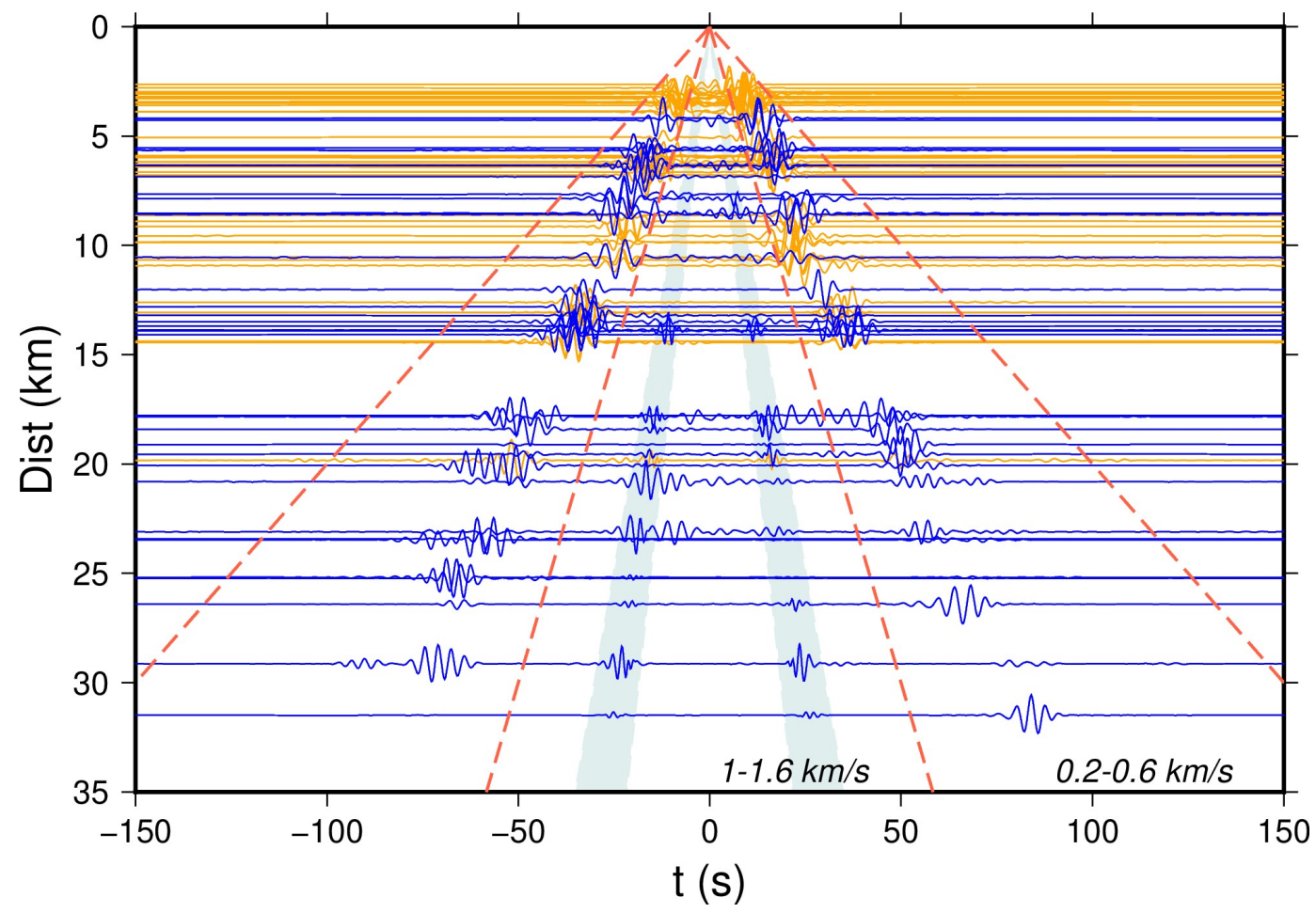

Figure 5.10: 75 station pair cross-correlation functions with clear signals computed using the pressure gauges (blue) or seismic vertical components (orange) of the OBSs, plotted as a function of interstation distance, filtered at 1-20 s, showing an earlier arrival with a dominant period of 1-3 $\mathrm{s}$ and a velocity of 1-1.6 km/s, denoted by the blue shade, and a later arrival (fundamental mode Rayleigh/Scholte waves) with a dominant period of 3-6 s and a velocity of $0.2-0.6 \mathrm{~km} / \mathrm{s}$. 


\subsubsection{Correction for compliance and tilt noise}

When deploying OBSs, instead of digging a hole and burying the sensor into the ground, as with onshore seismometers, OBSs are dropped from the ship and sink to the seafloor with unknown conditions. Although some seismometers can level themselves after landing on the seafloor, the sensor can still be tilted by the water waves during recording. Considering the special conditions under the sea, OBSs may be affected by seawater and unstable sediment (Crawford and Webb, 2000). The data recorded by OBSs are usually noisier than land station data. Most power spectral densities show the data from the seafloor are noisier than the global average high-noise model (e.g.,Figure 5.5; Kuo et al., 2014; Flores et al., 2017).

One way to improve the data quality is to correct the compliance and tilt noise on the seismic vertical component data (Crawford and Webb, 2000). Compliance noise (Figure 5.11a) is from the seafloor deformation caused by the pressure change of the overlying water column. As the water moves (gravity wave, infragravity wave, current, tide, etc.), its weight changes and deforms the seafloor. Bottom currents move the sensor to be tilted from the vertical, and current noise recorded by horizontal components projects onto the vertical component (Figure 5.11b), which is called tilt noise (Webb, 1998). Usually, compliance noise is larger than tilt noise. Crawford and Webb (2000) proposed a method to remove the compliance noise by determining the coherence between the pressure and vertical sensors and subtracting the coherent part from the vertical sensor. On the other hand, they suggested removing the tilt noise by determining the coherence between the horizontal and vertical sensors and subtracting the coherent part from the vertical sensor. These two processes can greatly improve long period signals like earthquakes. For short periods, Bowden et al. (2016) and Yang et al. (2020) applied this method to retrieve the 1st overtone Rayleigh/Scholte waves from ambient noise data. Compliance removal on the vertical component suppresses the waves that have good coupling with seawater. Fundamental mode Rayleigh/Scholte waves couple better with the seawater, and therefore they are suppressed by the compliance removal; in this way the 1st overtone Rayleigh/Scholte wave can be retrieved (Bowden et al., 2016).

To apply the correction, several days among the deployment time are picked uniformly. The days with big earthquakes should be avoided because earthquake 
frequencies can dominate the data. Power spectral density of raw data and power spectral density of the data after tilt and compliance noise correction are plotted (e.g., Figure 5.14) to determine if a correction is required or not. Sometimes the power spectral density after correction is the same as the power spectral density of the raw data. In such a case, correction is unnecessary. In other situations, the frequency range to correct is determined according to the power spectral density plots. In this study, tilt and compliance noise correction is conducted for the whole period range for higher mode Rayleigh/Scholte wave observation.

(a)

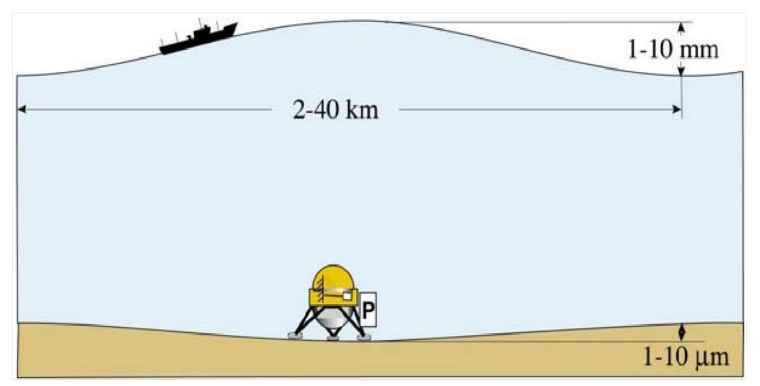

(b)

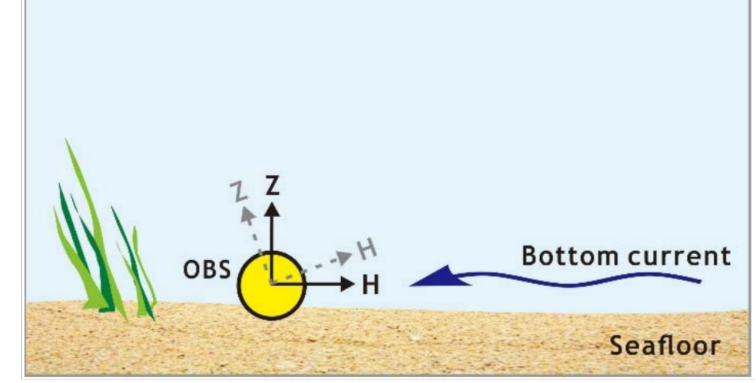

Figure 5.11: Cartoon illustration of two major sources of noise on OBS data. (a) An illustration of seafloor compliance noise. Assuming the water height changes 1-10 $\mathrm{mm}$, the seafloor would deform for 1-10 $\mu \mathrm{m}$ because of the overlying pressure change. (b) An illustration of tilt noise. $\mathrm{Z}$ denotes one vertical component, and $\mathrm{H}$ denotes two horizontal components. [From Kuo and Lin (2014)]

\section{Theory and workflow}

Pressure gauges equipped with OBSs are used to remove compliance noise, and seismic horizontal components are used to remove tilt noise. A pressure-vertical transfer function is calculated to remove compliance noise, and a horizontal-vertical transfer function is calculated to remove tilt noise (Crawford and Webb, 2000). Compliance noise cannot be corrected for the stations that do not have pressure channels.

One-sided auto-spectral density functions are estimated from the Fourier transforms of windowed sections of data. The auto-spectral density functions of the vertical component $G_{z z}$ and pressure gauge component $G_{p p}$ are given by

$$
G_{z z}(f)=\frac{1}{n \times N \delta t} \sum_{i=1}^{n}\left|Z_{i}(f)\right|^{2}
$$




$$
G_{p p}(f)=\frac{1}{n \times N \delta t} \sum_{i=1}^{n}\left|P_{i}(f)\right|^{2}
$$

and the cross-spectral density function of the vertical component and pressure gauge component $G_{z p}$ is given by

$$
G_{z p}(f)=\frac{1}{n \times N \delta t} \sum_{i=1}^{n} Z_{i}^{*}(f) P_{i}(f)
$$

where $Z_{i}^{*}(f)$ and $P_{i}(f)$ are fast Fourier transforms of data window $i$ for the $Z$ and $P$ components respectively, $n$ is the number of data windows, $N$ is the number of points of each data window, $\delta t$ is the sampling interval, the asterisk is complex conjugate (Bendat and Piersol, 2011). Each window is detrended and cosine tapered.

The coherence function is

$$
\gamma_{z p}(f)=\frac{G_{z p}(f)}{\sqrt{G_{z z}(f) G_{p p}(f)}}
$$

One then calculates the frequency-domain transfer function between $\mathrm{Z}$ and $\mathrm{P}$ channels:

$$
T_{z p}(f)=\gamma_{z p}(f) \times \sqrt{\frac{G_{z} z(f)}{G_{p} p(f)}}
$$

In the frequency domain, the noise in component $Z$ is linearly related to $P$ component through the transfer function. Subtracting the noise from $\mathrm{P}$ on $\mathrm{Z}$ :

$$
Z^{\prime}(f)=Z(f)-T_{z p}^{*} \times P(f)
$$

In this study, as both seismic vertical $(\mathrm{Z})$ and pressure gauge $(\mathrm{P})$ data are used for computing cross-correlation functions, both $\mathrm{Z}$ and $\mathrm{P}$ data are corrected from tilt and compliance noise. The horizontal components of on-land seismometers are usually named $\mathrm{N}$ and E. For OBSs, because the horizontal components cannot be orientated on the seafloor during deployment, they are named 1 and 2 instead of $\mathrm{N}$ and E. The workflow of the correction is shown in Figure 5.12: (1) Calculate the transfer function $T(Z, 1)$ between $Z$ and component 1 to obtain the noise from 1 on $Z$. Subtract $T(Z, 1)$ from $Z$, forming $Z^{\prime}$. (2) Since the noise source channels 1 and 2 may be coherent with one another, one must subtract the coherent effect on 2 from 
previous source 1. Otherwise, the transfer function (containing the spectrum of 2) between 2 and $Z^{\prime}$ would include the noise from 1 already removed, adding noise to $Z$. Therefore, this step is to compute the transfer function $T(1,2)$ between 1 and 2 components. Subtract the coherent part of 1 and 2 from 2, forming 2'. (3) Compute the transfer function $T\left(2^{\prime}, Z^{\prime}\right)$ and subtract it from $Z^{\prime}$ to take the noise out from $2^{\prime}$ on $Z^{\prime}$, forming $Z^{\prime \prime}$, which has been corrected from tilt noise. (4) Similar to (1) (2) (3), removing tilt noise on $P$, forming $P^{\prime \prime}$. (5) To subtract the coherent pressure signal, one calculates the transfer function $\mathrm{T}\left(\mathrm{P}^{\prime \prime}, \mathrm{Z}^{\prime \prime}\right)$ to obtain the noise from $\mathrm{P}^{\prime \prime}$ on $Z^{\prime \prime}$. Subtract $T\left(P^{\prime \prime}, Z^{\prime \prime}\right)$ from $Z^{\prime \prime}$, forming $Z^{\prime \prime \prime}$, which is corrected from tilt and compliance noise.

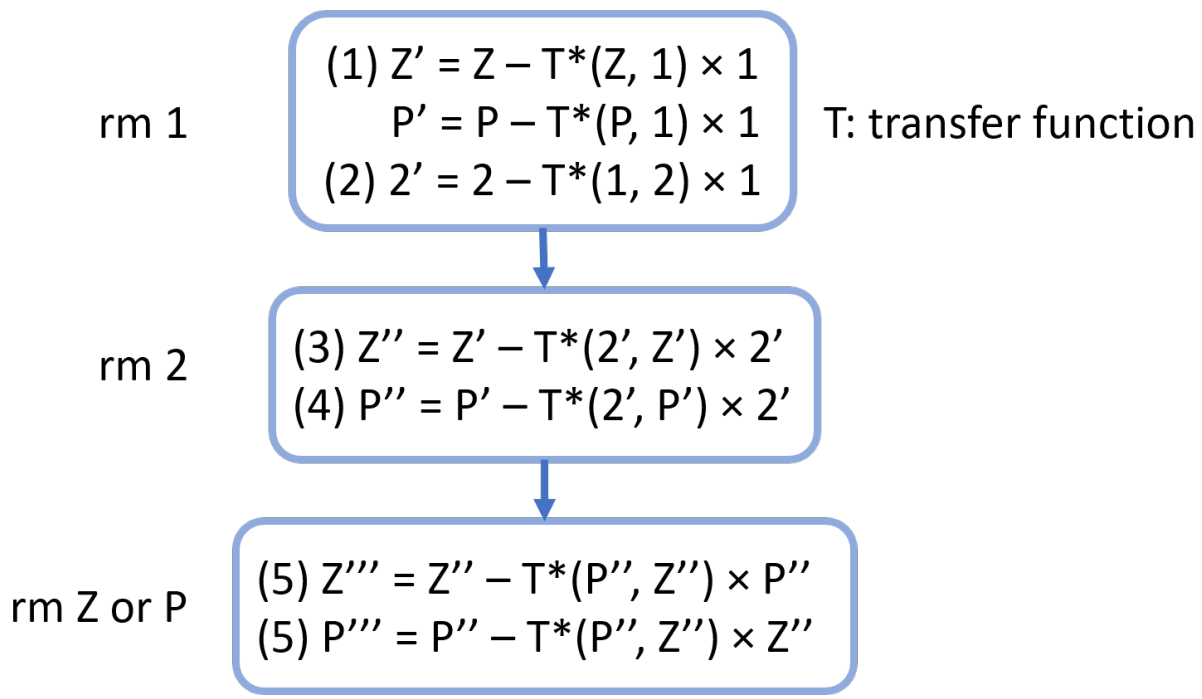

Figure 5.12: Workflow of tilt and compliance noise correction. The numbers (1) to (5) on the left of the equations correspond to that in the texts. Here 1 means one of the seismic horizontal channels, and 2 means the other seismic horizontal channel. Asterisk denotes the complex conjugation. rm stands for remove. The first step is to remove the effect of 1 on $\mathrm{Z}, \mathrm{P}$, and 2; the second step is to remove the effect of 2 on $Z$ and $P$; the last step is, if $Z$ will be used, remove $P$ effect on $Z$, or if $P$ will be used, remove the effect of $Z$ on $P$.

\section{The frequency range to correct}

Pressure $(\mathrm{P})$, seismic vertical $(\mathrm{Z})$, and the horizontal components are coherent among both noise and signal (e.g., microseism) periods (Figure 5.13). To avoid microseism signal being subtracted from $Z$, some corrections set the transfer function to 0 during the microseism signal period range (e.g., Crawford and Webb, 2000; Bowden et al., 2016). The P-Z coherence is strong during the secondary microseism period (Figure 5.13), but we only want to subtract the noise from the source channels (1, 
2, $\mathrm{P}$ components) to the vertical channel. To avoid subtracting the secondary microseism, Crawford and Webb (2000) set transfer function $\mathrm{T}(\mathrm{f})=0$ for frequencies above $0.1 \mathrm{~Hz}$. However, to retrieve the 1st overtone Rayleigh/Scholte waves, Yang et al. (2020) conducted the correction for the whole frequency range.

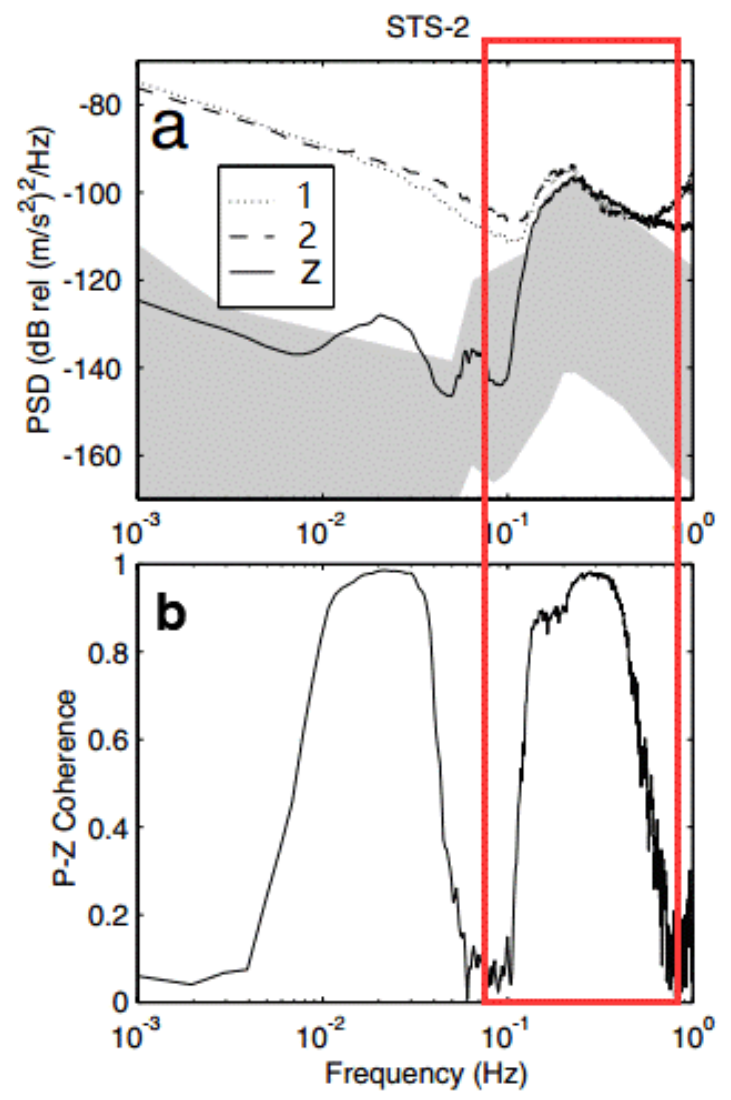

Figure 5.13: Power spectral density (a) and pressure-vertical coherence (b) from a seafloor seismometer/pressure gauge package (not from this study). The grey shade in the spectra shows the world noise bounds. The P-Z coherence in the red rectangle shows that $\mathrm{P}$ and $\mathrm{Z}$ are also coherent during the microseism period (around $0.125 \mathrm{~Hz}$ ). (a) Vertical and horizontal spectra. (b) Pressurevertical coherence. [From Crawford and Webb (2000)]

For some stations in this study, if we set $\mathrm{T}(\mathrm{f})=0$ during the microseism period range, the cross-correlation computed by the corrected data is the same as the cross-correlation computed by uncorrected data (Figure 5.14). This is because the frequency of the noise caused by seawater in our study region is lower than the frequency range of the microseism. Therefore, the noise correction cannot improve the signal (short-period) in this study.

Even if the correction does not appear to improve data quality, the earlier arrivals can be retrieved when the correction is conducted on the whole frequency range 
(Figure 5.15). Fundamental mode Scholte waves couple well with seawater since they propagate along with the interface of seawater and solid earth. Channel $\mathrm{P}$ records signals from the water directly, and the correction removes the coherent part of $\mathrm{P}$ and $\mathrm{Z}$ from $\mathrm{Z}$. Consequently, if the correction is conducted on the whole frequency range, it would suppress the fundamental mode Scholte waves at Z. When the fundamental mode is suppressed, the 1st overtone would be enhanced and emerge during cross-correlation computation. The reason is that the fundamental mode Scholte waves couple better with seawater. Removing signals that are coherent with the pressure gauge also removes the fundamental mode Scholte wave signals, and thus the 1st overtone can be emphasized (Bowden et al., 2016). As shown in Figure 5.15, when all the frequency range is corrected, the earlier arrivals (the blue waveform) emerge, and there is no fundamental mode Scholte wave. The earlier arrivals may be higher mode Scholte waves.

\section{The higher mode Scholte wave}

In this study, the compliance and tilt noise correction does not improve the data quality (Figures 5.14 and 5.15). Instead, the correction is used to test the coupling of the observed waves with the solid earth. If the waves couple well with the solid earth, they can be used to study the underground structure.

The earlier arrivals in Figure 5.10 show a velocity close to the speed of sound wave in the water. To test if they couple well with the solid earth instead of just traveling in water, correction of tilt and compliance noise is conducted on the seismic vertical components of 12 station pairs from which the latter arrivals (fundamental Scholte waves) are well retrieved, but the earlier arrivals are not observed. The compliance noise correction subtracts the coherent signal between the pressure and vertical channels from the vertical channel, which can suppress the signal that couples well with seawater. If the faster waves observed by the pressure-derived cross-correlations (Figure 5.10) emerge on the vertical channel after correction, the waves must couple well with the solid earth instead of the seawater. This is because the waves traveling only in the seawater should be removed by the compliance noise removal. In the results shown in Figure 5.16, the cross-correlations derived by compliance corrected data show the early arrivals similar to the ones in Figure 5.10, and the latter arrivals are suppressed. This suggests that the early arrivals couple well with solid earth and can be used to study the earth structure. 

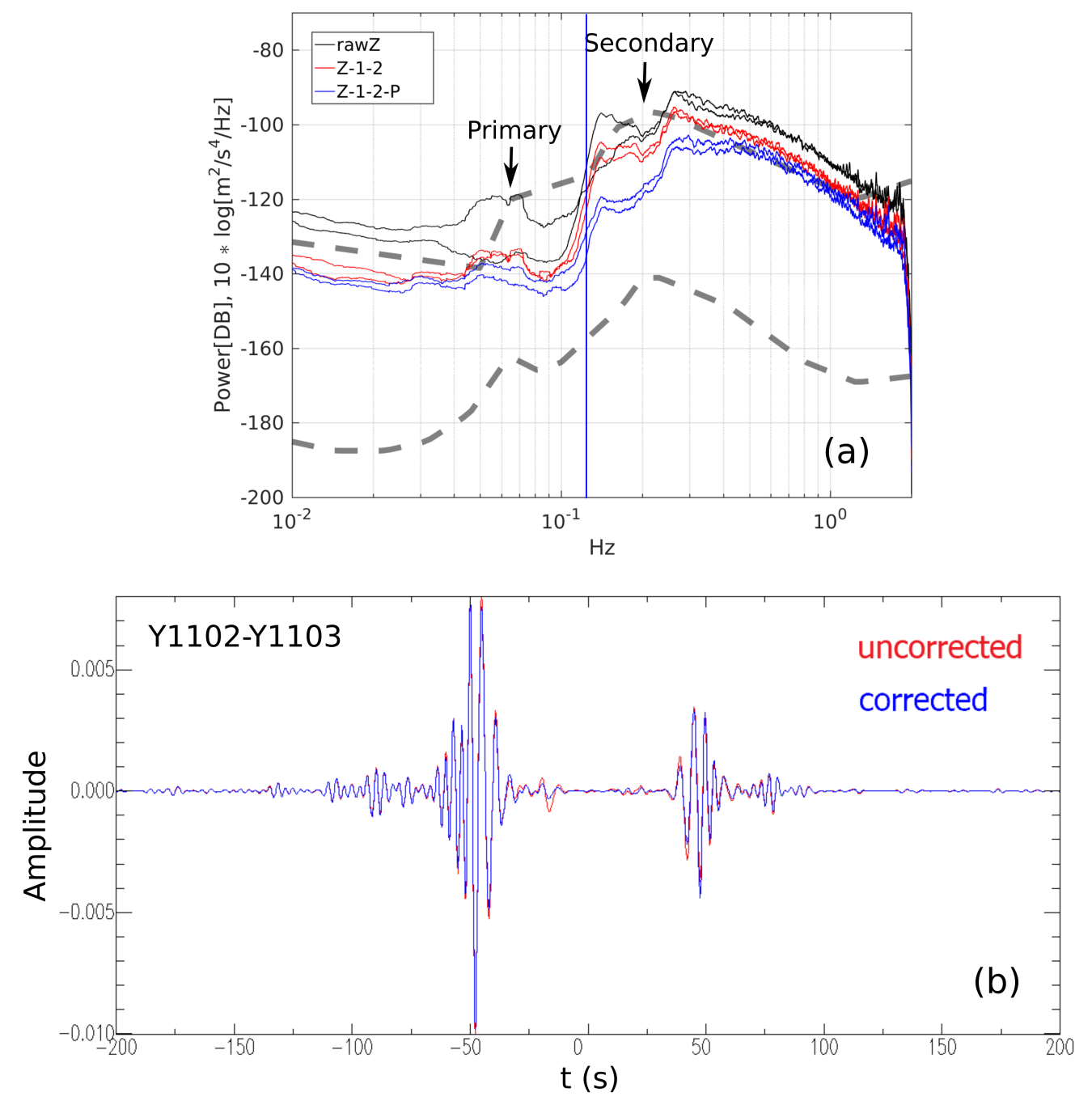

Figure 5.14: Power spectral density and waveform comparison of cross-correlations computed by uncorrected data and corrected data of station pair Y1102-Y1103. Data on each day is cut into half-day segments for computing the transfer function. Here power spectral density of one-day data is shown as an example. (a) Power spectral densities of raw data (black), the data after tilt noise correction (red), and the data after tilt and compliance noise correction (blue). The vertical blue line marks the highest frequency $(0.11 \mathrm{~Hz})$ to correct. Correction for frequencies $>0.11 \mathrm{~Hz}$ would decrease the secondary microseisms. Therefore, we only correct for the frequencies $<0.11$ Hz. (b) Waveform comparison. The red waveform is the stack of cross-correlations computed by uncorrected data; the blue waveform is the stack of cross-correlations computed by corrected data. They are similar. 


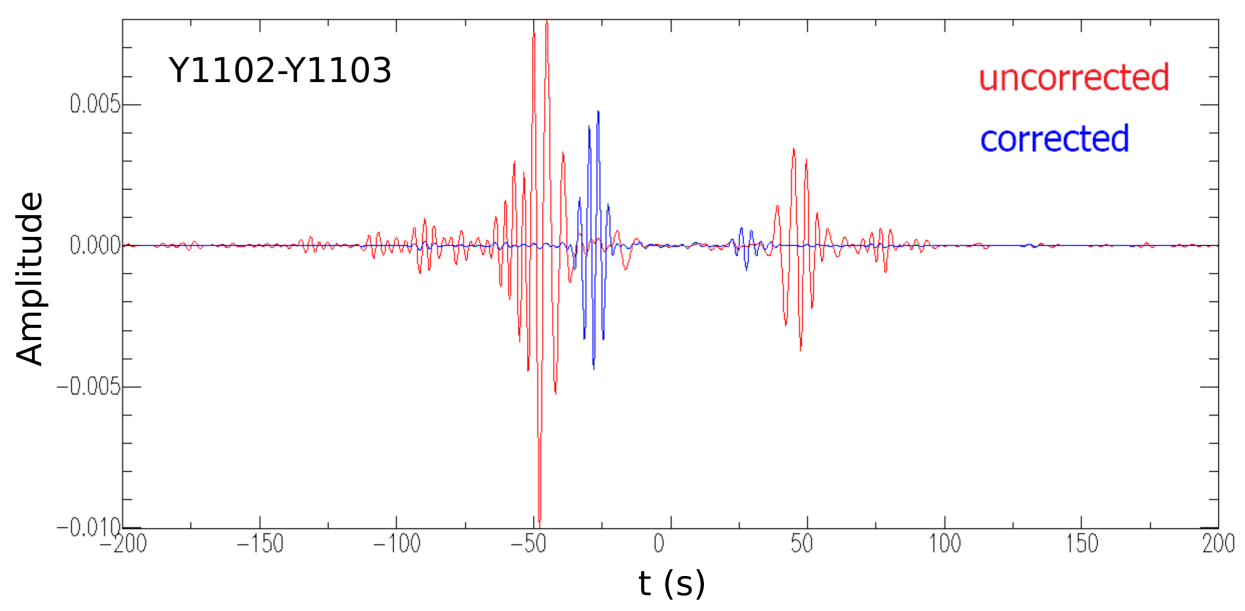

Figure 5.15: Similar to Figure 5.14b, but all the frequency range is corrected. Only the later arrivals (fundamental mode Scholte waves) are retrieved in the cross-correlation computed by uncorrected data. In contrast, in the cross-correlation computed by the corrected data, the latter arrivals vanish, and the earlier arrivals are retrieved, which might be the higher mode Scholte waves.
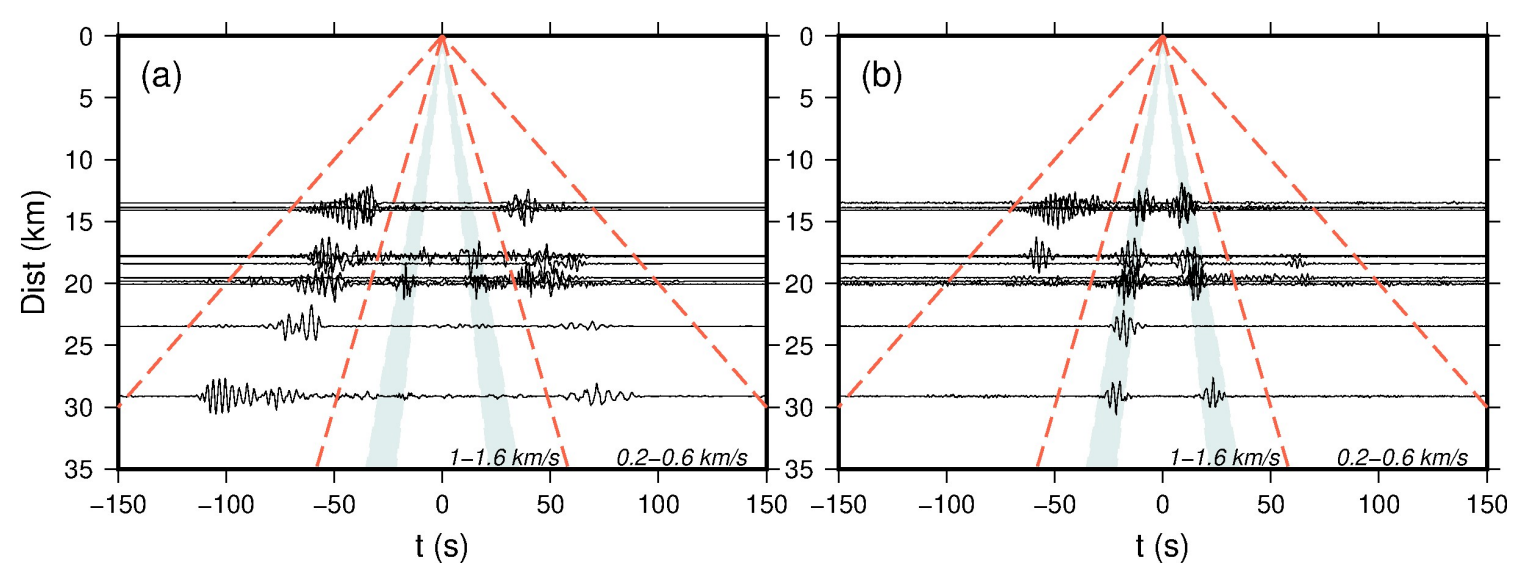

Figure 5.16: Comparison of cross-correlation functions computed by raw data and compliance corrected data. (a) cross-correlations computed by raw vertical component data. (b) cross-correlations computed by tilt and compliance noise corrected data. After the tilt and compliance noise correction, the latter arrivals (fundamental mode Scholte waves) are suppressed, and the early arrivals become much clearer. This indicates the early arrivals couple better with the solid earth. 


\subsection{Results}

\subsubsection{Phase velocity dispersion curves and 1D inversion}

\section{Measurement and average}

The cross-correlation functions are input into a program Frequency-Time Analysis (FTAN; Levshin et al., 1992; Levshin and Ritzwoller, 2001), designed for processing seismic surface wave records through spectral and frequency-time analysis for phase velocity measurements. The phase velocity measurement method is described in Section 2.6. Phase velocities of both the earlier arrivals (possible higher mode Scholte waves) and the latter arrivals (fundamental mode Scholte waves) are measured. The longest period that can be measured is restricted to two times the interstation distance (Bensen et al., 2007; Luo et al., 2015). Measurements with low signal-to-noise ratio $(<10)$ are not used. A forward model of phase velocity dispersion curve based on a shear wave velocity model at site S002 (Figure 5.3; Kuo et al., 2015) and an associated phase velocity dispersion curve computed by all the cross-correlation functions in the corresponding region are used as references to unwrap the phase (picking one branch from the measurement). Figure 5.17 shows an example of phase unwrapping. Figure 5.18 displays the phase velocity measurements from all the station pairs, and Figure 5.19 shows the station-pair averaged phase velocities. 


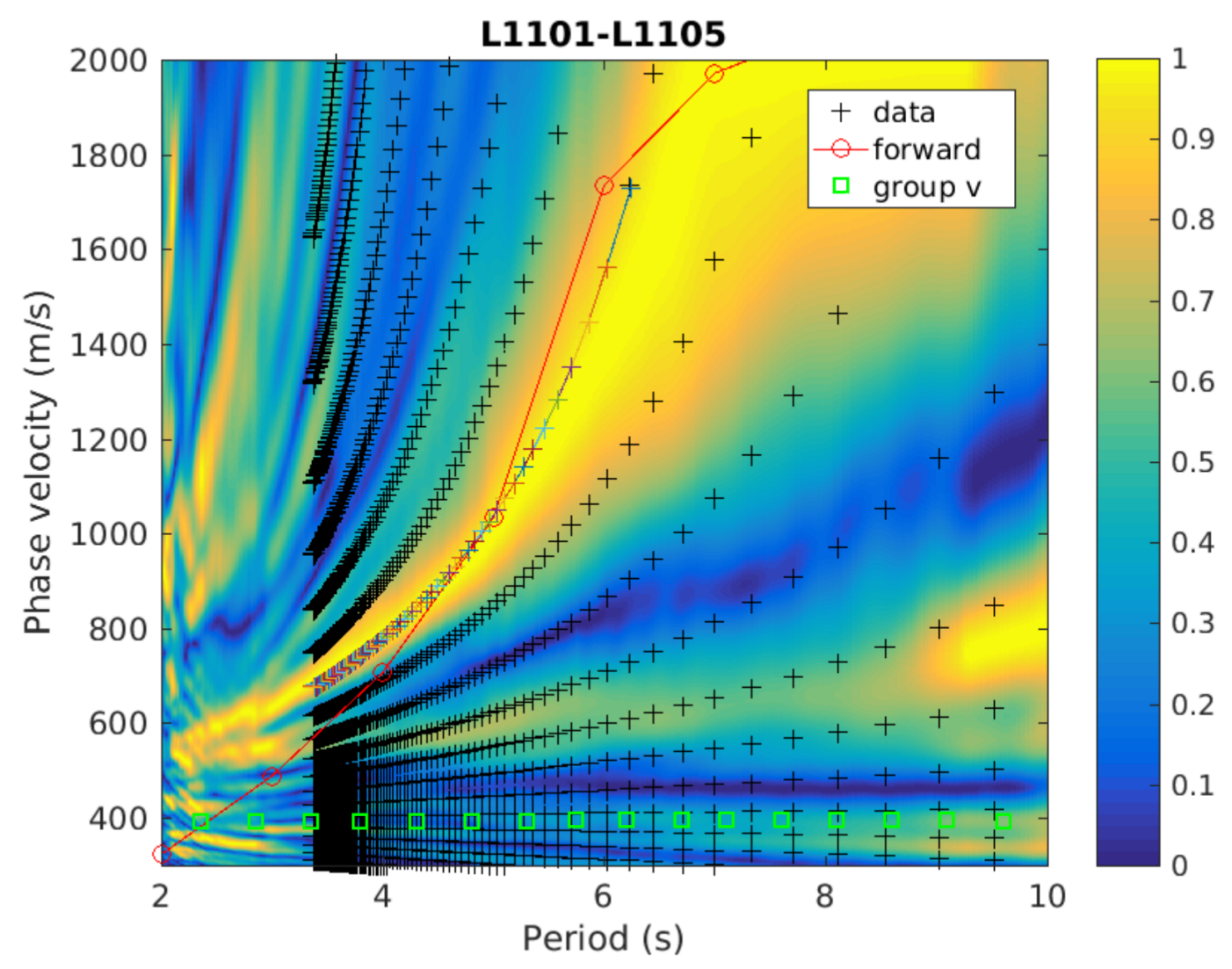

Figure 5.17: Unwrapping the phase of measurement on L1101-L1105. Black crosses display the measured phase velocities with different cycles, and colored crosses are the chosen phase velocities. Each station pair is picked separately. The background colors show associated phase velocity dispersion energy using all the fundamental mode Scholte waves, following the method proposed by Park et al. (1998). The red curve with circles showing its samples denotes the phase velocities predicted by a reference model at site S002 close to our study region (Figure 5.3; Kuo et al., 2015). 


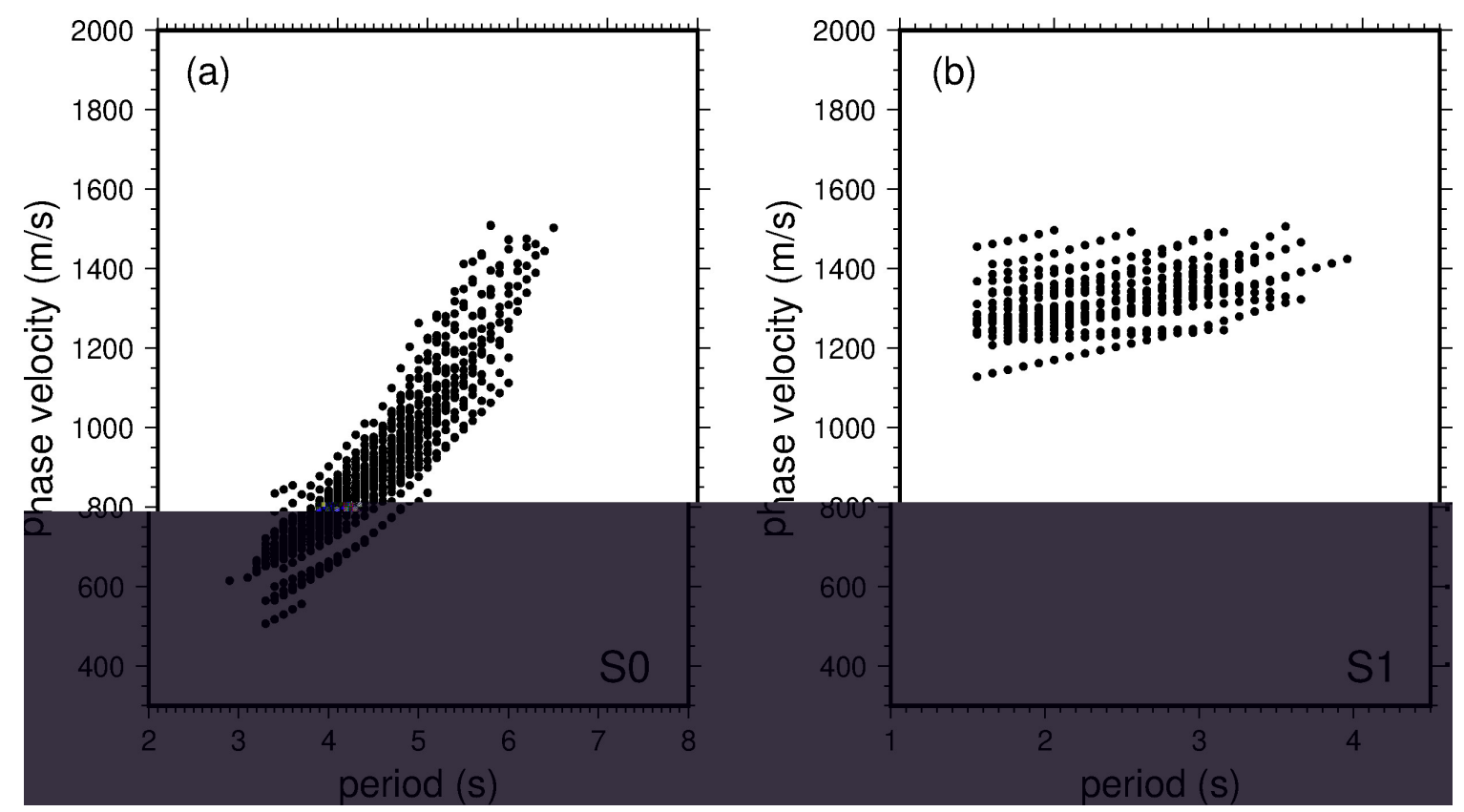

Figure 5.18: Phase velocity dispersion curves measured from all the cross-correlation functions. (a) Dispersion curves of fundamental mode Scholte waves. (b) Dispersion curves of the possible higher mode Scholte waves. Here the dispersion curves are after phase unwrapping (Figure 5.17).

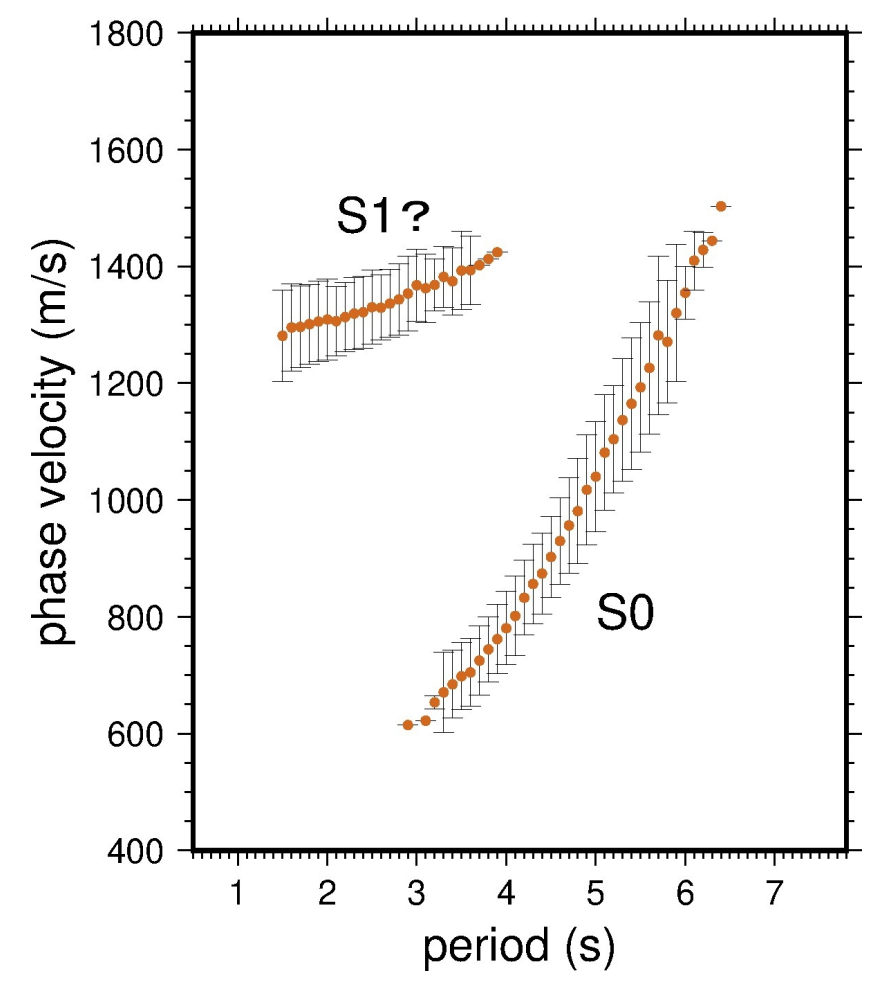

Figure 5.19: Averaged phase velocity dispersion curves of fundamental mode Scholte waves (S0) shown in Figure 5.18a and the possible 1st overtone Scholte waves (S1) shown in Figure 5.18b, with standard deviations as the errors. 


\section{Three sub-regions}

The study region is divided into three sub-regions (Figure 5.20) according to the measured phase velocities and tectonics: southwest edge of Okinawa Trough, the center of the study region, and south of the rifting axis. The station-pair path coverages (Figure 5.20) and dispersion curves (Figure 5.21) in the corresponding sub-regions are displayed with different colors. The phase velocities in the south and northwest edge regions are slower than the phase velocities in the center region, but not by more than one standard deviation. As the station coverage in the south region is only near the rifting axis, to better compare the velocity difference from either side of the rifting axis, the station pairs in the center region close to the rifting axis are marked by orange (Figures 5.20 and 5.21a). The orange dispersion curves (center) are still faster than the red dispersion curves (south). The dispersion curve measured from pair YB01-YB02 (Figure 5.20) crossing the volcanic outcrop (2-0 Ma; Sibuet et al., 1998; Wang et al., 1999) shows higher velocity than other dispersion curves. The averaged dispersion curves (Figure 5.21b) in the three sub-regions are used to invert for three 1-D shear wave velocity structures in the corresponding sub-regions. 


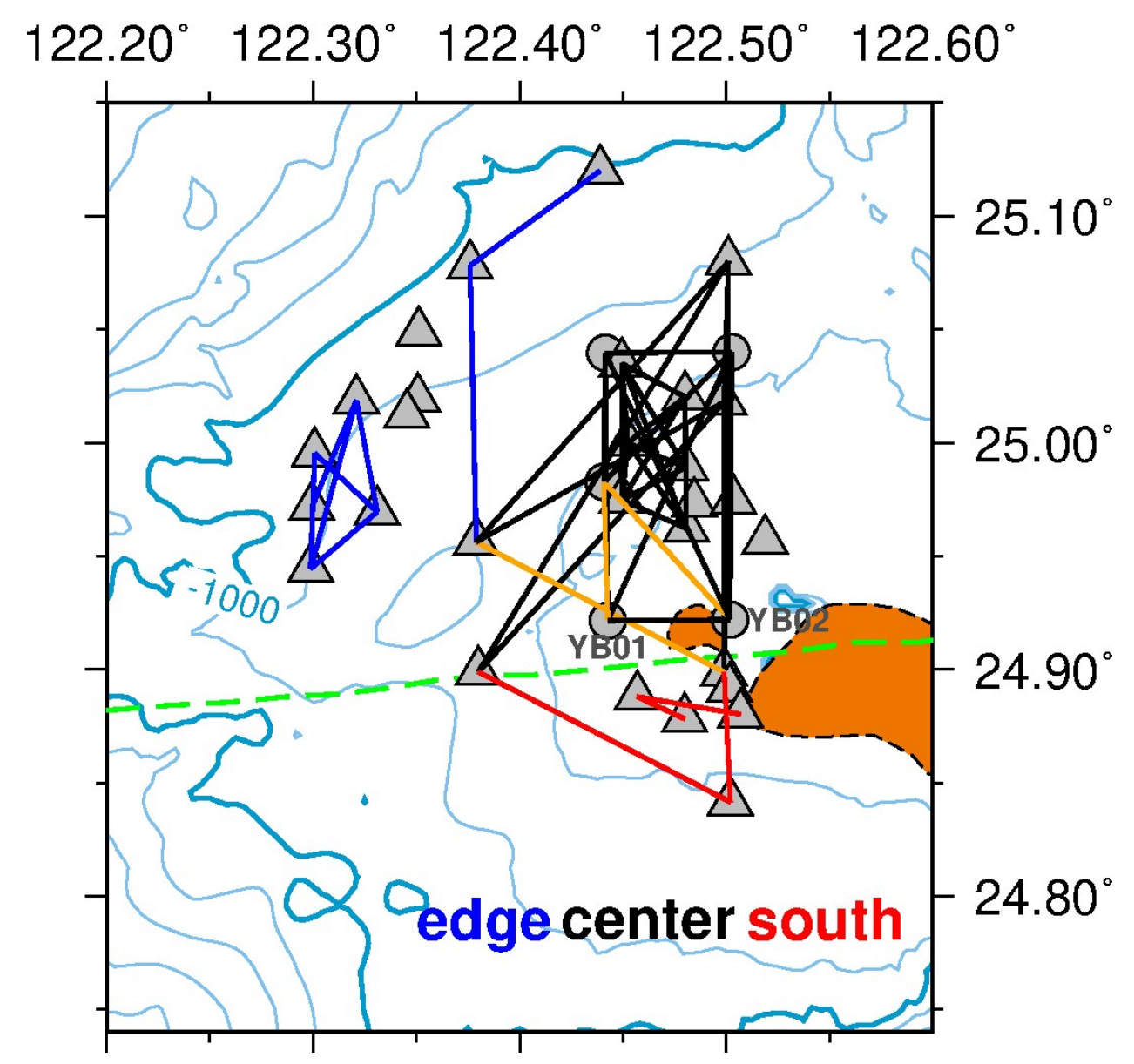

Figure 5.20: Station-pair path coverage in the northwest edge of the study region (blue), center of the study region (black), and south of the rifting axis (red). The green dashed line indicates the rifting axis. To better compare the velocities in center and south regions, the stations pairs in the center region that are close to the rifting axis are denoted by orange. The orange shades mark the volcanic outcrops or slightly buried volcanism in the study region (Sibuet et al., 1998). 

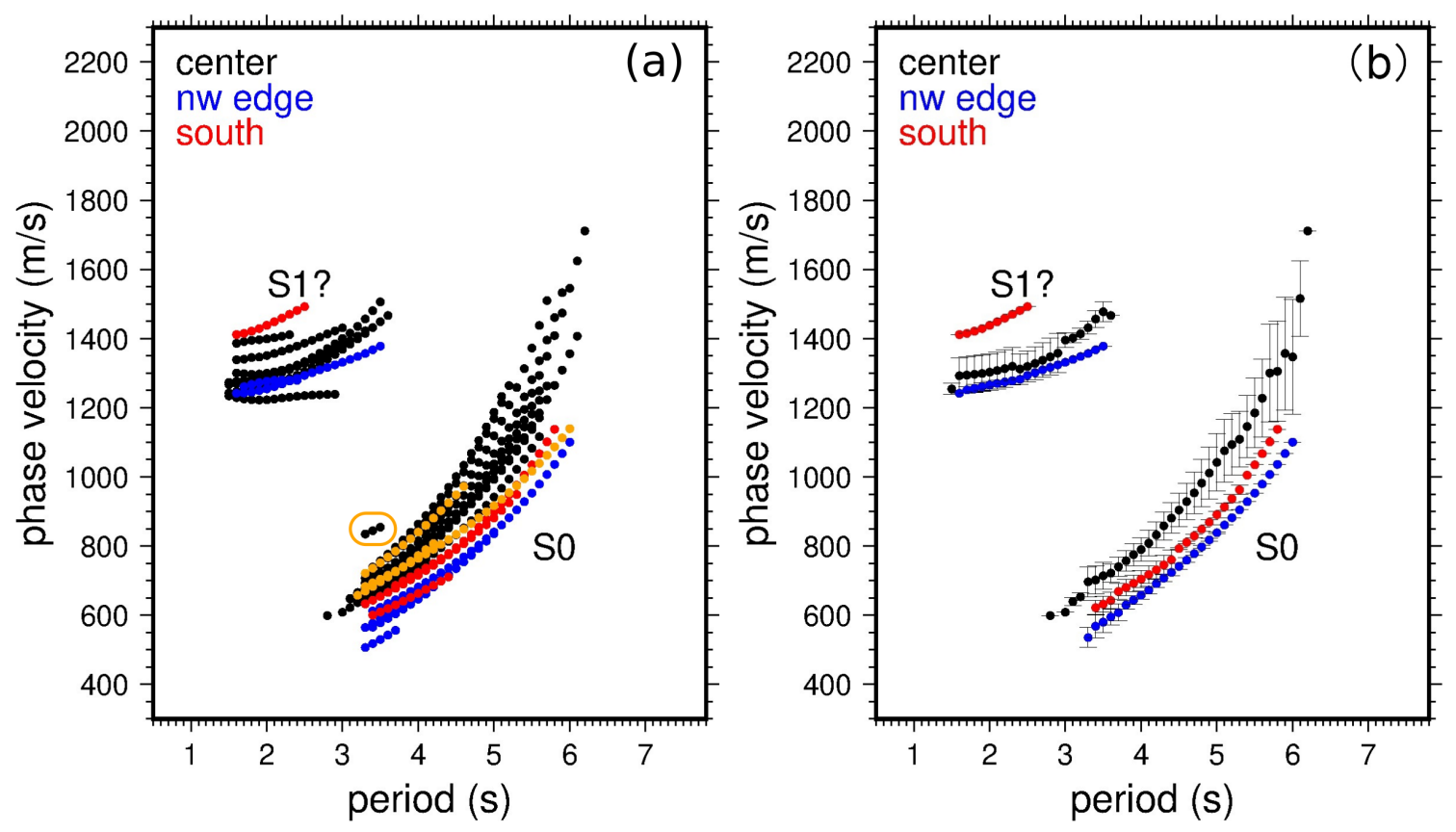

Figure 5.21: Phase velocity dispersion curves measured from the two arrivals in Figure 5.10 in the three sub-regions: center, northwest edge (nw edge), and south. The colors of the dispersion curves correspond to the ray path colors in Figure 5.20. (a) Dispersion curves measured from the latter arrivals - fundamental mode Scholte waves (S0) and the earlier arrivals - possible 1st overtone Scholte waves (S1). The orange dispersion curves are from the center region near the rifting center (Figure 5.20). The short black dispersion curve marked by an orange circle is associated with pair YB01-YB02 crossing the volcanic outcrop (Figure 5.20), and shows higher velocity than the other dispersion curves. (b) Averaged phase velocity dispersion curves of those in (a), with standard deviations as the errors. The standard deviations in the south and northwest edge regions are small because these two regions do not contain many dispersion curves. 
The averaged fundamental mode dispersion curves in the three sub-regions (Figure 5.21b) are used as the observations for 1D shear wave velocity inversions (Figures 5.22-5.24). The inversion is conducted using a Bayesian method (Guo et al., 2016), which is discussed in Section 2.6. A Markov Chain Monte Carlo method (Guo et al., 2016; Yang et al., 2020) is used to search for the Vs models. Phase velocity dispersion curves are forward-modeled based on the Vs models using Computer Programs in Seismology (Herrmann, 2013). During the inversion, 40,000 initial models are proposed within a wide range of shear velocities (Table 5.2). Vp values and layer depths are fixed according to previous reflection seismic studies (Klingelhoefer et al., 2009; Wang et al., 2004) that cross the study region of this chapter. Density is calculated based on Vp according to a relationship $\rho=1.85+0.165 V p$ (Christensen and Shaw, 1970; Zha and Webb, 2016). For the three sub-regions, Vs search ranges are the same, and the prior settings are the same except for the depths of seawater and sediment. Seawater depths in the three sub-regions are obtained by averaging water depths of the OBSs used in each corresponding region. Following Guo et al. (2016) and Yang et al. (2020), we first conduct 40,000 searches following a uniform distribution within the model space. The 40,000 models are then used to generate an initial Gaussian proposal distribution. Afterwards, every 10,000 proposed models (searches) are used as historical samplings to adjust a covariance matrix of the Gaussian proposed distribution. Subsequent samplings then draw from this Gaussian distributed prior. After 100,000 searches, the last 2,000 samplings are used to construct the posterior probability density function (PDF).

If many models with a wide range of values fit the data with high PDF values, it suggests that the shear wave velocity is not well constrained by the observed data. Inversions for the Vs models in the center region (Figure 5.22), northwest edge (Figure 5.23), and south region (Figure 5.24) show that the observed data do not have resolution of shear wave velocity below $3.6 \mathrm{~km}$ (basement). Below the basement, a wide range of Vs values have high PDF, indicating that observations in this study can only resolve Vs structure down to the basement.

A joint inversion using the fundamental mode and the 1st overtone Rayleigh/Scholte waves in the center region is tested, assuming the earlier arrivals are the 1st overtone (Figure 5.25). However, the data cannot be fit when the earlier arrivals are assumed to be the 1st overtone, and are used together with the fundamental mode 
Table 5.2: The prior setting for Vs inversion. The velocity and density values are the same for the three sub-regions, but the water and sediment depths in the three sub-regions vary. The first three columns show the depths of layers in the center, northwest edge (nw edge), and south regions. The depth here denotes the bottom depth of the layer beneath sea level.

\begin{tabular}{|l|l|l|l|l|l|}
\hline Depth $(\mathrm{km})$ - center & nw edge $(\mathrm{km})$ & South $(\mathrm{km})$ & $\mathrm{Vp}(\mathrm{km} / \mathrm{s})$ & $\mathrm{Vs}(\mathrm{km} / \mathrm{s})$ & $\rho\left(\mathrm{g} / \mathrm{cm}^{3}\right)$ \\
\hline 1.39 (water) & 1.16 & 1.455 & 1.5 & 0 & 1.02 \\
1.4 & 1.17 & 1.465 & 1.8 & $0.05-1$ & 2.0 \\
2.31 (sediment) & 2.08 & 2.245 & 2.0 & $0.05-2.5$ & 2.0 \\
3.6 (basement) & 3.6 & 3.6 & 3.25 & $1.0-3.52$ & 2.386 \\
6.8 (upper crust) & 6.8 & 6.8 & 5.0 & $2.05-4.48$ & 2.675 \\
9.0 & 9.0 & 9.0 & 6.0 & $2.45-4.48$ & 2.84 \\
11.0 (lower crust) & 11.0 & 11.0 & 6.75 & $3.95-5.48$ & 2.964 \\
15.0 (upper mantle) & 15.0 & 15.0 & 8.1 & $3.95-5.48$ & 3.327 \\
\hline
\end{tabular}

during the inversion. This suggests that the earlier arrivals might not be the 1st overtone of Rayleigh/Scholte waves. Similar inversion tests for the other two subregions, and tests assuming that the earlier arrivals are 2nd or 3rd overtones, are conducted (Appendix C), but the data still cannot be fit. The observed earlier arrivals, dominated at 1-3 s period band, may be mixed by multiple higher mode Scholte waves traveling in the sub-seafloor sediments (Nolet and Dorman, 1996). Therefore, they are not able to be used during inversion with the assumption of one particular mode. The earlier arrivals are finally not used for inversion. 

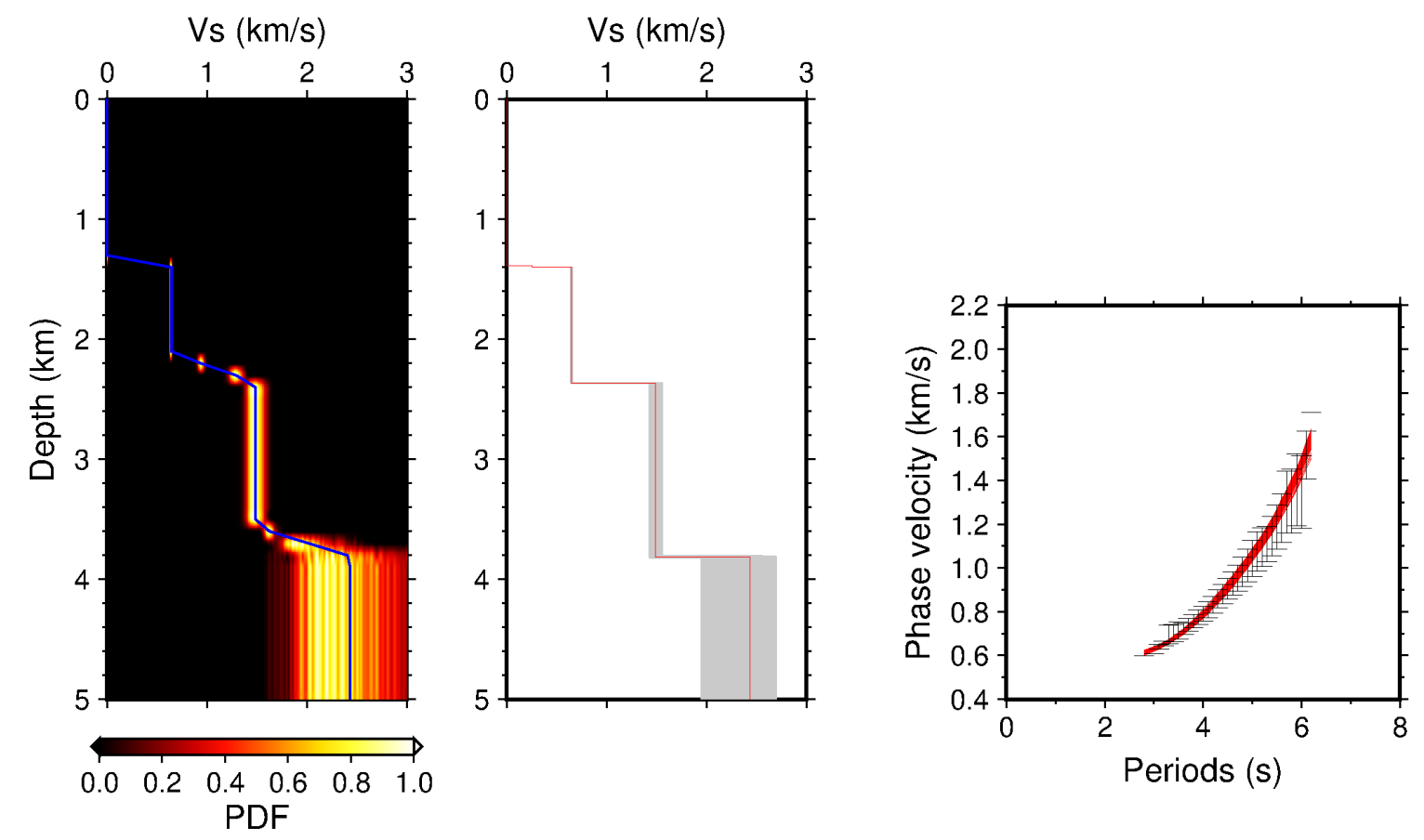

Figure 5.22: Vs inversion in the center region using the fundamental mode dispersion curve (the black dispersion curve $\mathrm{S} 0$ in Figure 5.21b), and corresponding data fit. Left: posterior distributions of the final 2,000 models from the inversion. The color scale represents the normalized posterior probability density function (PDF). The blue line indicates the mean of the 2,000 models. Middle: Vs range of the final 2,000 models (grey shade) and their mean (red line). Right: data fit; the observed fundamental mode phase velocities are shown by error bars, while the red shade shows the range of phase velocities forwarded by the 2,000 Vs models. 

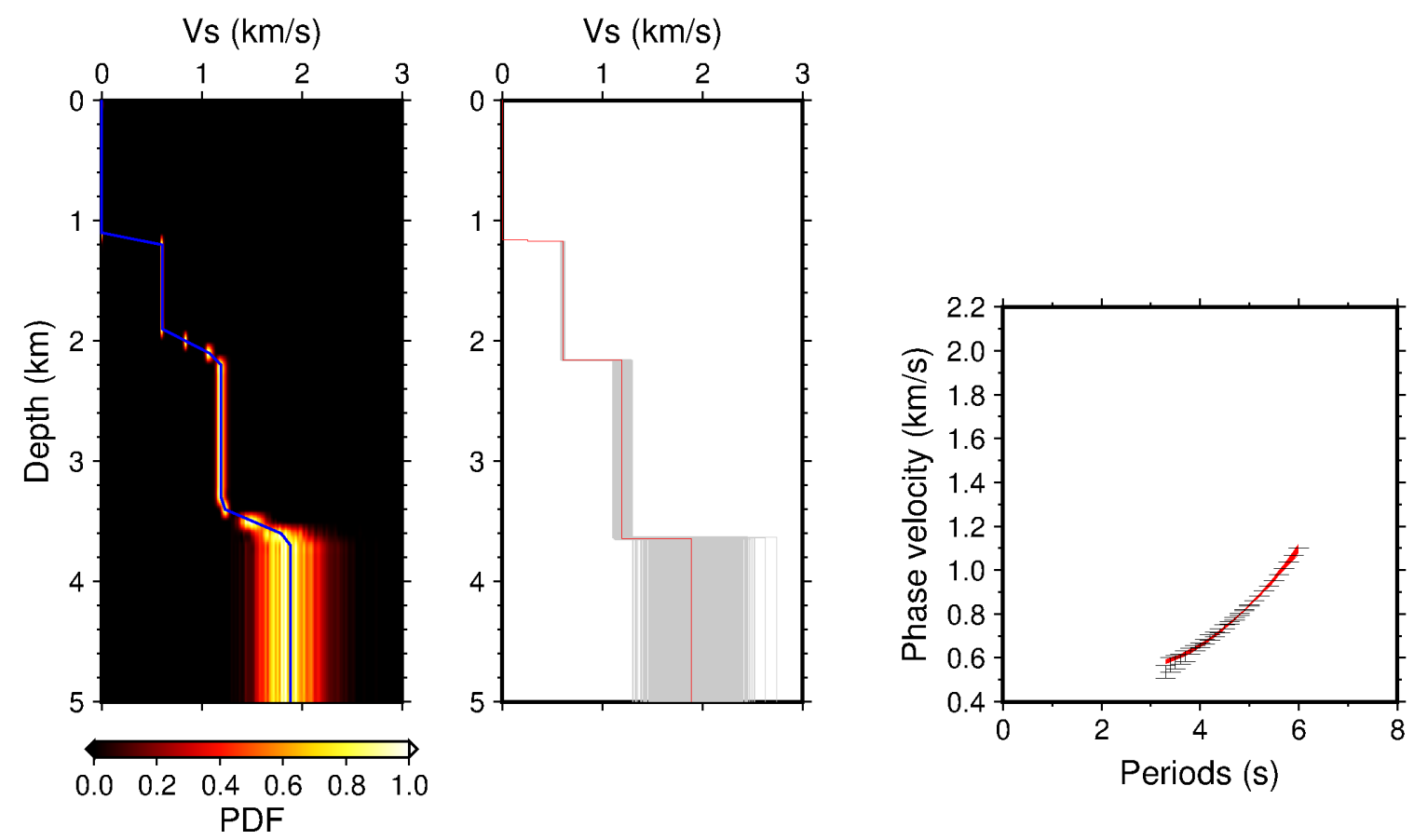

Figure 5.23: Vs inversion in the northwest edge region using the fundamental mode dispersion curve (the blue dispersion curve S0 in Figure 5.21b), and corresponding data fit.
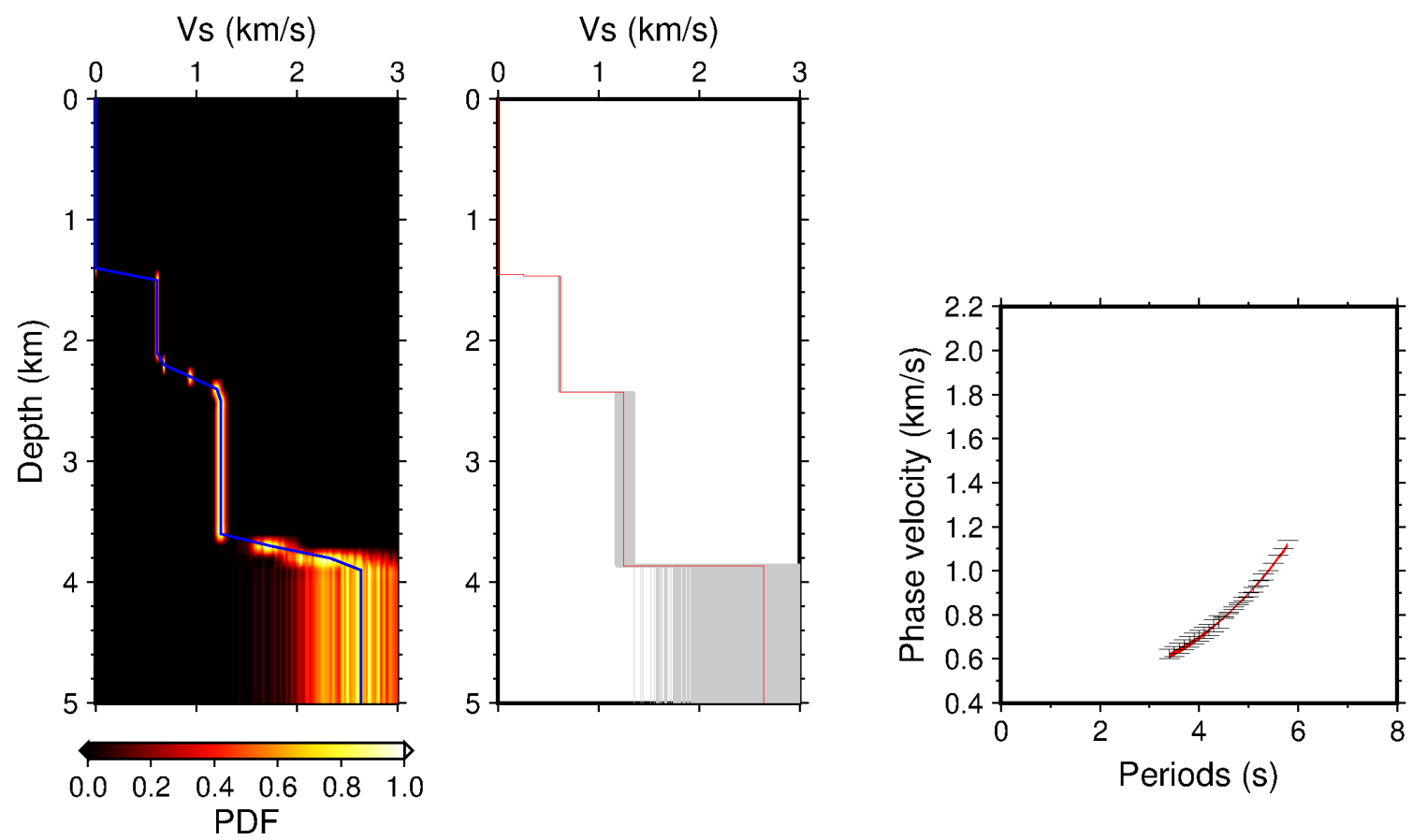

Figure 5.24: Vs inversion in the south region using the fundamental mode dispersion curve (the red dispersion curve S0 in Figure 5.21b), and corresponding data fit. 

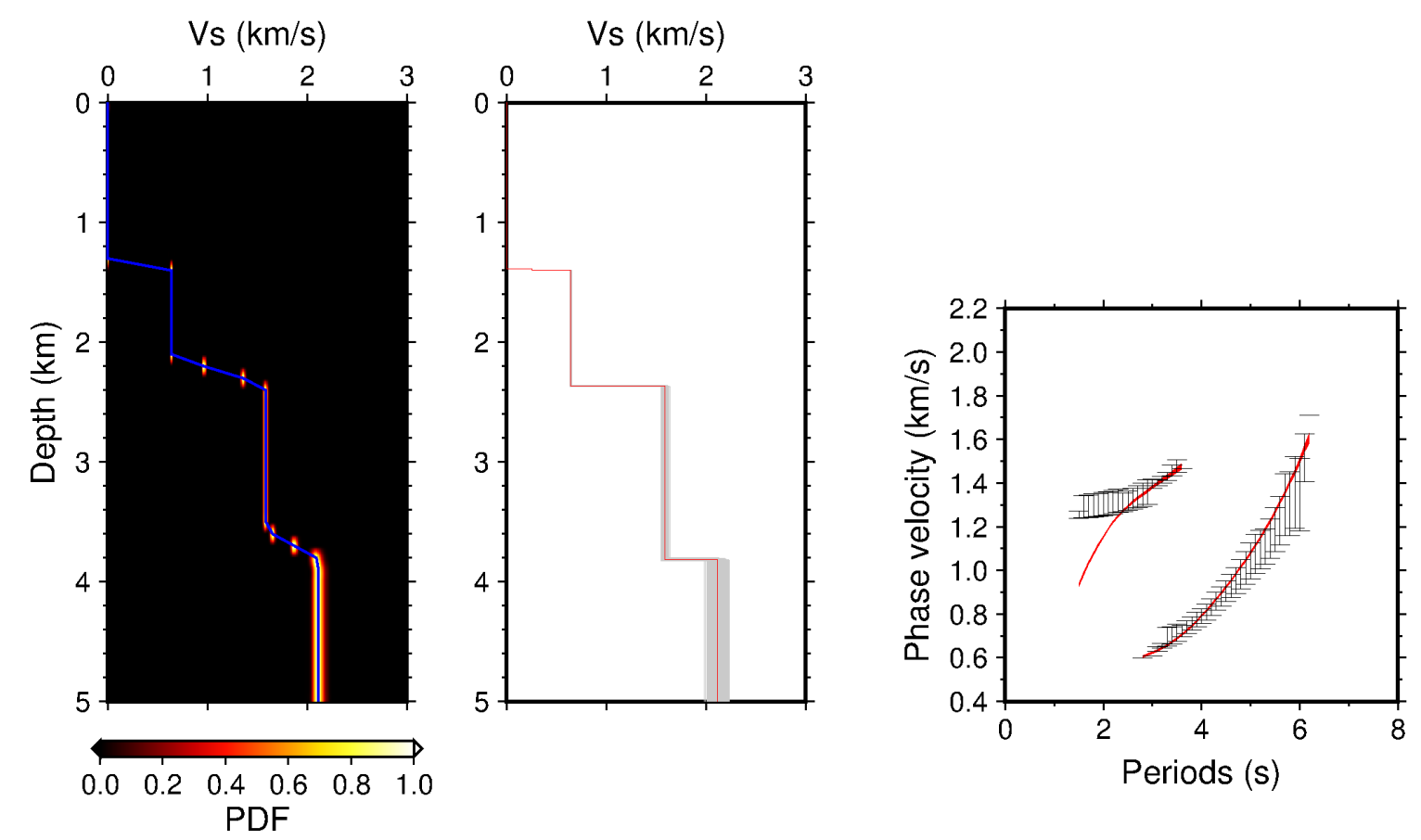

Figure 5.25: Vs inversion in the center region using the fundamental mode and assumed 1st overtone dispersion curves (the black dispersion curves S0 and S1 in Figure 5.21b), and corresponding data fit. The higher mode cannot be fit by the observation. 
Table 5.3 presents the mean Vs and corresponding uncertainties in the three subregions at sediment, basement, and upper crust layers. The uncertainties are calculated using the weighted standard deviation,

$$
\sigma=\sqrt{\frac{\sum_{i=1}^{N} \omega_{i}\left(v_{i}-\bar{v}\right)^{2}}{\frac{M-1}{M} \sum_{i=1}^{N} \omega_{i}}}
$$

where $N$ is the number of models $(N=2,000), M$ is the number of non-zero weights, PDF values are used as weights $\omega_{i}, v_{i}$ is the shear velocity of one of the 2,000 models, and $\bar{v}$ is the mean shear velocity. We have not taken into account the errors in the phase velocity curves.

Table 5.3: Mean Vs and corresponding double standard deviations in the three sub-regions at sediment, basement, and upper crust layers, plotted in Figure 5.26.

\begin{tabular}{llll}
\hline layer & sediment & basement & upper crust \\
\hline $\begin{array}{l}\text { center region } \\
\text { mean Vs }\end{array}$ & 0.645 & 1.488 & 2.431 \\
$2 \times$ standard deviation & 0.006 & 0.0896 & 0.6084 \\
\hline northwest edge & & & \\
mean Vs & 0.604 & 1.193 & 1.884 \\
$2 \times$ standard deviation & 0.0104 & 0.0444 & 0.3096 \\
\hline south region & & & \\
mean Vs & 0.613 & 1.246 & 2.643 \\
$2 \times$ standard deviation & 0.0104 & 0.0478 & 0.6272 \\
\hline
\end{tabular}


Figure 5.26 shows the mean Vs models of the three sub-regions with uncertainties. The shear wave velocity in the center region of the basement layer is higher than that in the northwest edge and south regions. The center region and south region are divided by the rifting axis (Figure 5.20). The velocity difference on the two sides of the rifting axis suggests an asymmetric structure of the back-arc basin, or a low shear velocity in the south region near the rifting axis. We acknowledge that we do not have many stations in the south region.

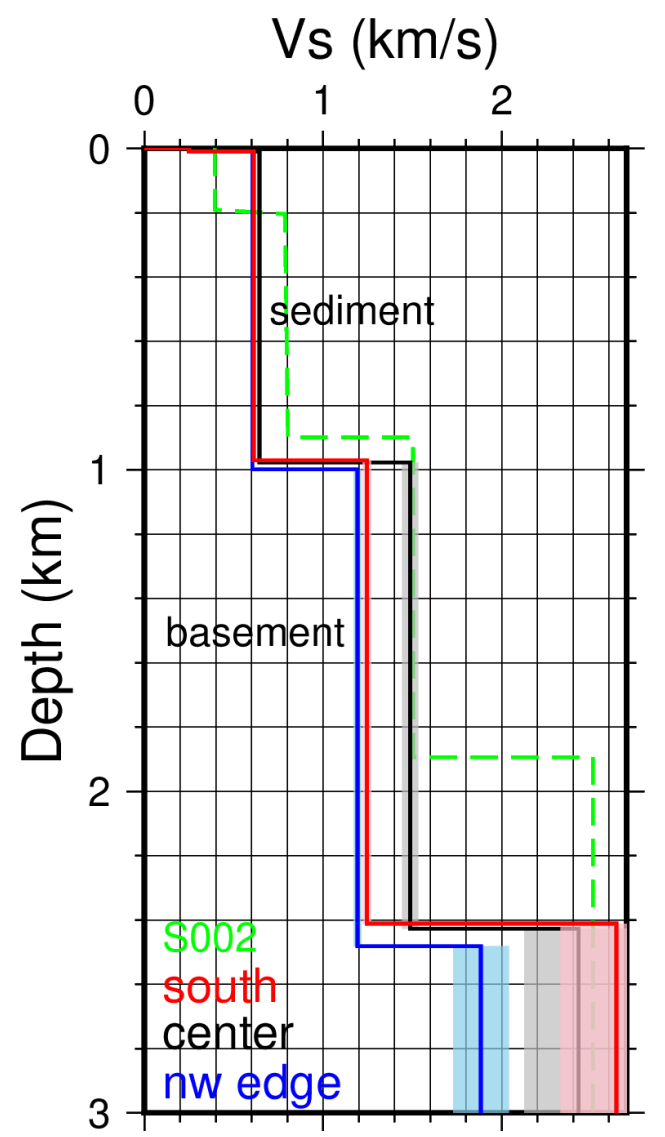

Figure 5.26: Mean Vs models of the three sub-regions plotted from the seafloor (water layer is not plotted). Corresponding dispersion curves refer to Figure 5.21b. The shades (e.g., the grey shade behind the black Vs model) show the corresponding uncertainties. As a reference, the green dashed line shows the Vs model at site S002 proposed by Kuo et al. (2015). 


\subsection{Discussion}

\subsubsection{Higher mode Rayleigh/Scholte waves}

Tilt and compliance noise is corrected in an attempt to retrieve higher mode Scholte waves. Earlier arrivals are observed (Figure 5.16) after the noise correction, but the inversion cannot fit the data if the earlier arrivals are assumed to be a single type of higher mode Rayleigh/Scholte waves (Figure 5.25).

Here higher mode Rayleigh/Scholte wave dispersion curves are forward-modeled (Figures 5.27-5.29), based on the inverted velocity models (Figure 5.26) which only use the fundamental mode dispersion curves as observations, for the three sub-regions. For all the three regions, the dispersion curves of the earlier arrivals do not fit with the forward-modeled dispersion curves of any higher mode Rayleigh/Scholte waves. The higher mode Rayleigh/Scholte wave dispersion curves are steeper than the dispersion curves of the earlier arrivals. The earlier arrivals must couple better with the solid earth than water because they are retrieved after compliance removal. It is possible that the observed earlier arrivals dominant at $1-3 \mathrm{~s}$ period are mixed with multiple high modes of Rayleigh/Scholte waves in the sediments of the seafloor (Nolet and Dorman, 1996). Previous studies (Bowden et al., 2016; Yang et al., 2020) have applied compliance correction to retrieve the 1st overtone Rayleigh/Scholte waves which were successfully included in shear velocity inversions. However, their observations were longer than $3 \mathrm{~s}$ period. Different modes of Rayleigh/Scholte waves at longer periods ( $>3 \mathrm{~s}$ ) may not easy to mix. 


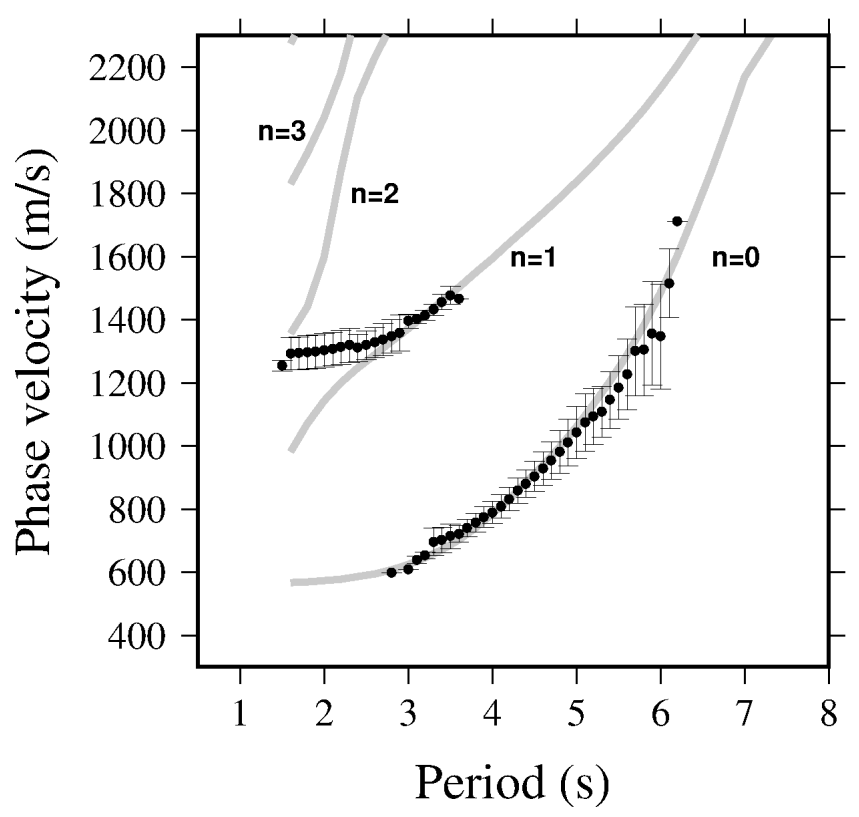

Figure 5.27: Comparison of forward-modeled dispersion curves and observed dispersion curves in the center region. The grey lines show the forward-modeled dispersion curves based on the inverted Vs model (the black line in Figure 5.26), from fundamental mode ( $n=0)$ Rayleigh/Scholte wave to the fourth overtone $(n=4)$. The black dots with error bars show the averaged phase velocity dispersion curves in the center region (Figure 5.21b).

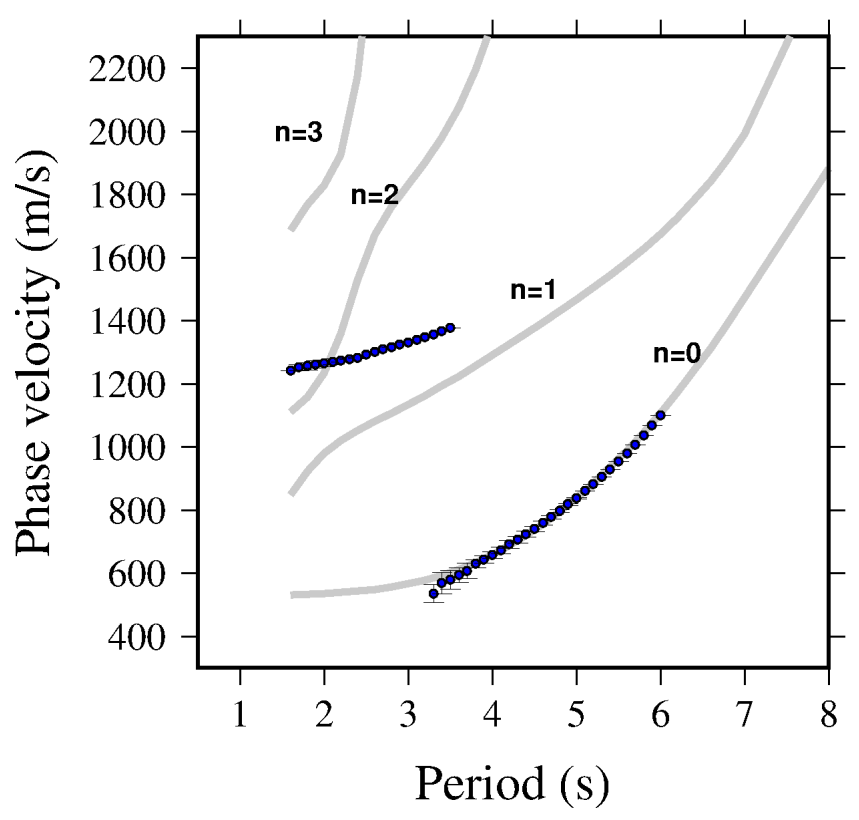

Figure 5.28: Comparison of forward-modeled dispersion curves and observed dispersion curves in the northwest edge region. The grey lines show the forward-modeled dispersion curves based on the inverted Vs model (the blue line in Figure 5.26). The blue dots with error bars show the averaged phase velocity dispersion curves in the northwest edge region (Figure 5.21b). 


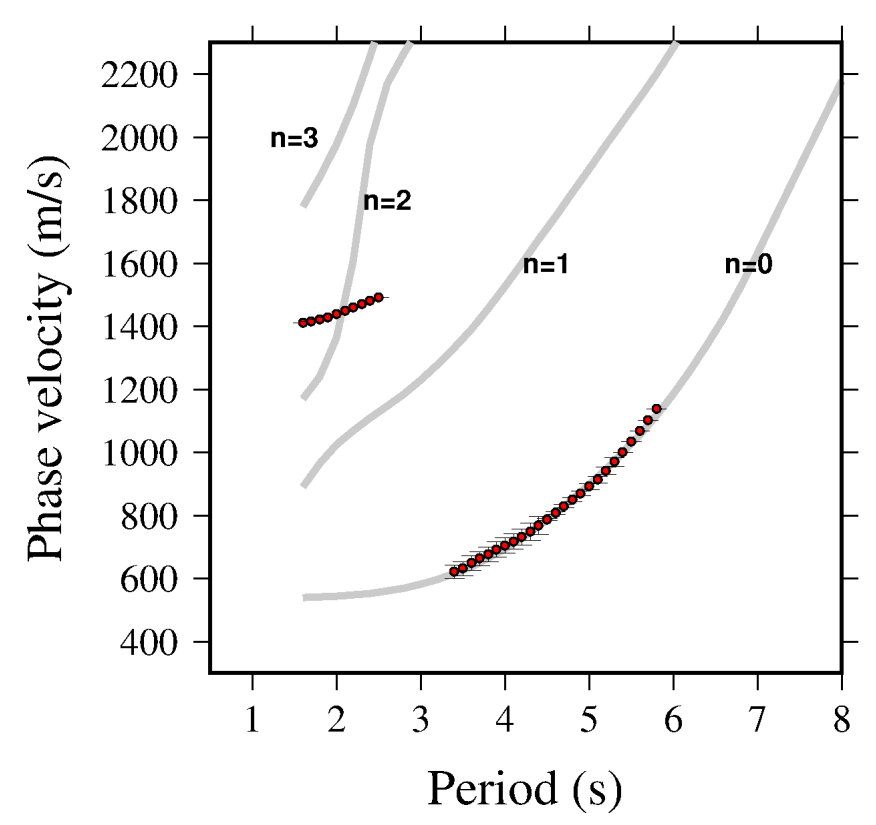

Figure 5.29: Comparison of forward-modeled dispersion curves and observed dispersion curves in the south region. The grey lines show the forward-modeled dispersion curves based on the inverted Vs model (the red line in Figure 5.26). The red dots with error bars show the averaged phase velocity dispersion curves in the south region (Figure 5.21b). 


\subsubsection{Sensitivity kernels}

Sensitivity kernels of fundamental mode Rayleigh/Scholte wave phase velocities are computed based on the inverted shear velocity models in the three sub-regions (Figure 5.30). The kernels show sensitivity down to $4 \mathrm{~km}$, indicating the inversion results down to $4 \mathrm{~km}$ are valid (Figure 5.26). This is in accordance with the inversion resolution (e.g., Figure 5.22) which indicates that the models with a wide range of Vs values have high PDF values (the yellow models) below $3.6 \mathrm{~km}$.
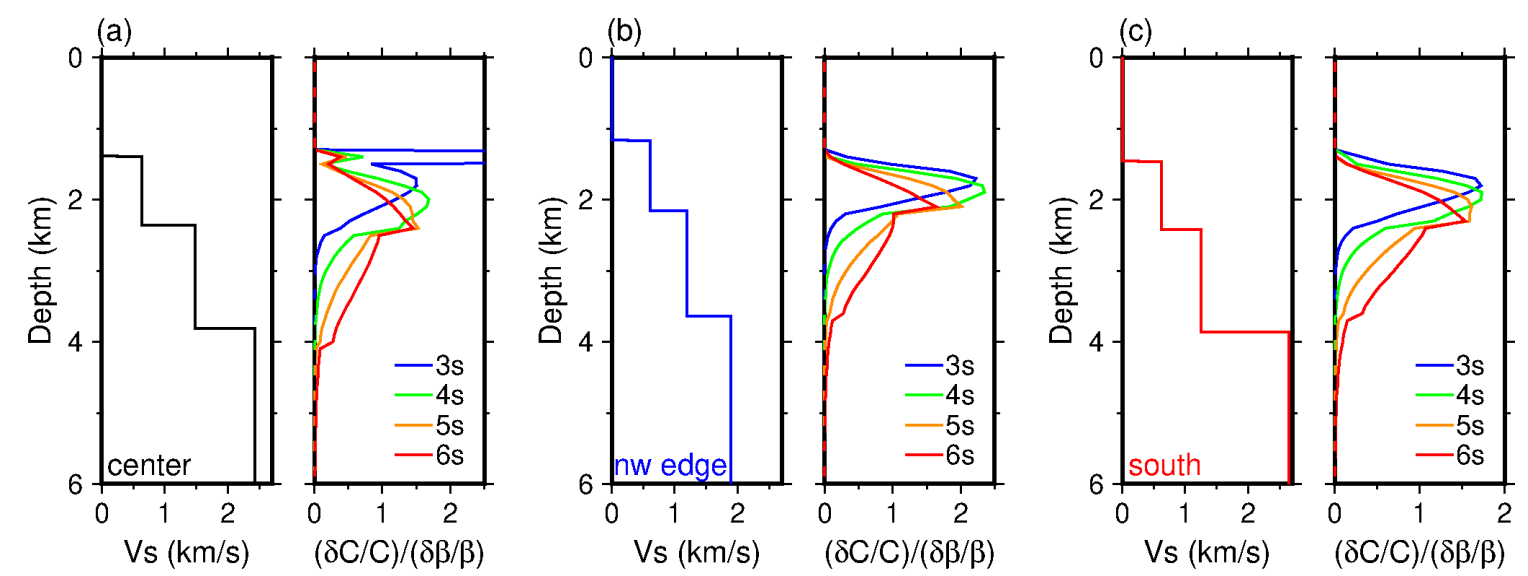

Figure 5.30: Sensitivity kernels of fundamental mode Rayleigh/Scholte wave phase velocities, based on the inverted shear velocity models in the center region (a), northwest edge region (b), and south region (c). 


\subsubsection{Volcano}

In the study region, refraction channel (Sibuet et al., 1998) was characteristic of a low-energy environment with muddy deposits. The sedimentation rate is extremely high, possibly in excess of $3 \mathrm{~m} / \mathrm{k} . \mathrm{y}$. (Salisbury, 2002). The sedimentary succession consists of one lithostratigraphic unit, which is characterized by homogeneous, bioturbated clayey silt with isolated sandy intervals. From the smear slide analyses, the nonbiogenic sediment fraction is dominated by quartz and feldspar grains and abundant detrital carbonate clasts.

The ray path YB01-YB02 passes through a volcanic outcrop (Figure 5.20), aged at 2-0 Ma (Sibuet et al., 1998; Wang et al., 1999), and its dispersion curve (Figure 5.21) shows higher velocity than other station pairs. This could be due to a cooled magma chamber, which would result in higher velocity than extruded lava or melt. The cooled magma chamber may be deeper than the depths we can constrain, but the velocity above a cooled magma chamber would still be high. South of this volcanic outcrop, the dispersion curves (red curves in Figure 5.21) show much slower velocities. The cooled magma chamber under pair YB01-YB02 must be within a small region (diameter $\sim 3 \mathrm{~km}$ ), which does not extend to the south region. The dispersion curve is very short because the inter-station distance is short, with only three samplings at short periods ( $3.3 \mathrm{~s}, 3.4 \mathrm{~s}$, and $3.5 \mathrm{~s})$, but the benefit of short inter-station distance is that it can provide information for small structures, as a dispersion curve reflects an average of materials between the two stations. The dispersion curve of YB01-YB02 is therefore largely determined by this small volcanic outcrop (diameter $\sim 3 \mathrm{~km}$ ), without the perturbation of others.

\subsubsection{High heat flow}

The center and south regions are divided by the back-arc rifting axis. The reason that the velocity in the south region is lower than the velocity in the center region might be related to high heat flow in the south region (red circle in Figure 5.2). High heat flow in the south region would result in more melts/fluids in surrounding materials, leading to lower shear wave velocity.

Previous studies have linked asymmetric spreading to thermal effects (Barker and Hill, 1980; Mantovani et al., 2001). Stern and Benson (2011) proposed the litho- 
sphere in the east of the Taupo Volcanic Zone is thinner than the west due to the subduction, which causes heat output in the east to be higher than that in the west. In our study region, the heat flow in the south region may be a consequence of asymmetric spreading of the back-arc. Previous studies (Oshida et al., 1992; Doo et al., 2019) showed an asymmetric magnetic anomaly distribution in our study region - the magnetic anomaly in the south is higher than that in the north. However, because we do not have a high resolution lithosphere depth in the study region, we do not have sufficient evidence to support the asymmetric spreading hypothesis.

Arai et al. (2017) provided seismological evidence for the structure of the southern Okinawa Trough, to the east of our study region, where they suggested a symmetrical rifting. While their seismic profile did not cross any hydrothermal vents, the deployment in this study covers volcanic outcrop (Figure 5.20), and the deployment is beside a high heat flow spot (red circle in Figure 5.2). Within our study region, the heat flow in the south of the basin is higher than that in the north of the basin (Chen et al., 2020), which might be a consequence of asymmetric spreading. We acknowledge that we do not have much constraint in the south region because of the limited station coverage, and the low velocity in the south region may be only near the rifting center. However, comparing the phase velocities from the station pairs that are near the rifting axis from north and south of the rifting axis (the orange and red dispersion curves in Figure 5.21), the velocity in the north of the rifting axis is still higher than the velocity in the south, suggesting the velocity structure is asymmetric along the rifting axis. Figure 5.31 summarises the findings of this study.

\subsection{Conclusions}

This study provides information on shear wave velocities in the upper crust of the southwestern Okinawa Trough. This study focuses on a small region $\left(\sim 0.2^{\circ} \times 0.3^{\circ}\right)$, with a dense deployment (2-10 km inter-station distance), providing evidence for small structures (volcanic outcrop with diameter $\sim 3 \mathrm{~km}$ ). Under the small volcanic outcrop, a cooled magma chamber is more likely than a hot one because of the high velocity. This study finds the seismic velocity in the north of southwestern Okinawa Trough rifting axis is faster than the seismic velocities in the south of 


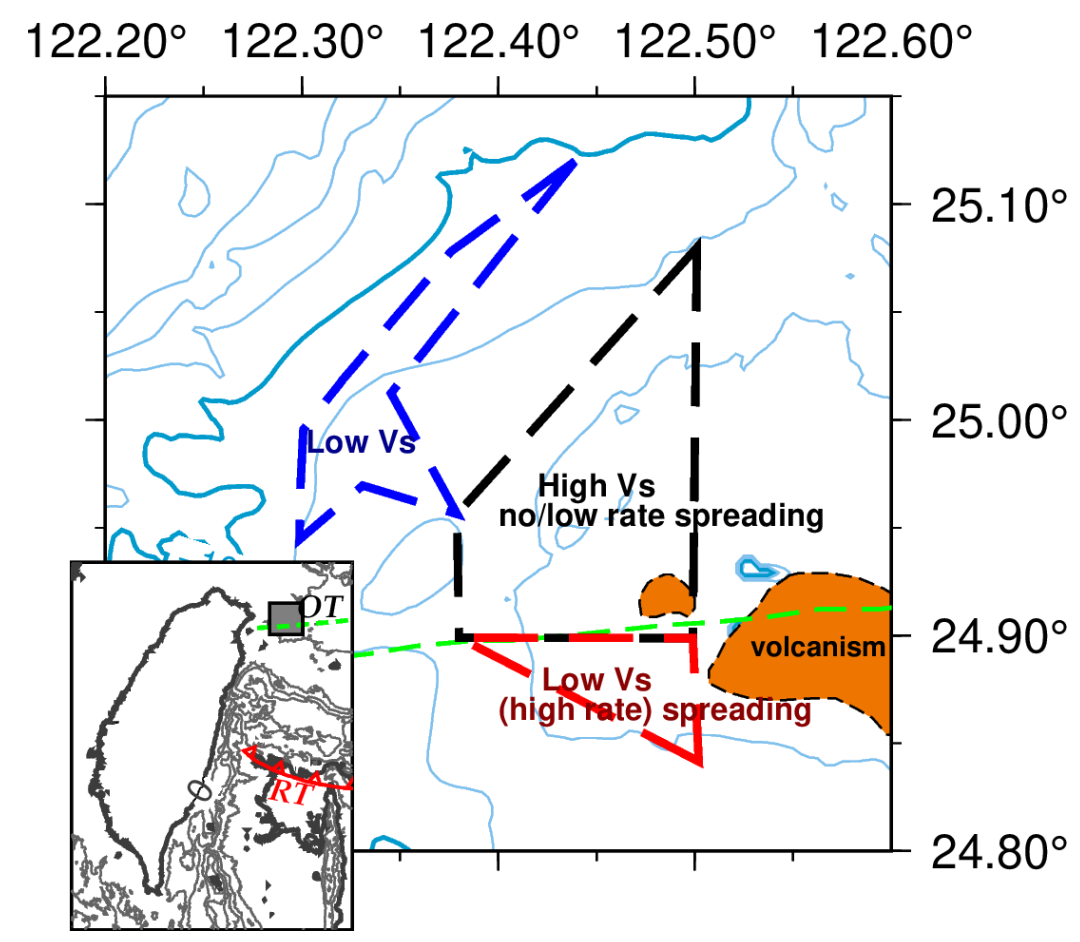

Figure 5.31: Spatial distribution of velocity findings and tectonic interpretation. The station coverage in the three sub-regions are outlined by different colored dashed lines. The green dashed line marks the back-arc rifting center. OT, Okinawa Trough; RT, Ryukyu Trench.

the rifting axis and northwest edge of the study region. The possible causes of the velocity difference are the high heat flow in the south region and/or asymmetric back-arc extension. We do not have additional information in the northwest edge to interpret the lower velocity.

In summary, this chapter:

1. Tests parameters in MSNoise, aiming to retrieve high SNR direct arrivals (Rayleigh/Scholte waves).

2. Uses both seismic vertical channels and pressure gauges equipped with OBSs. From both seismic vertical channels and pressure gauges, clear Rayleigh/Scholte waves can be retrieved.

3. Applies compliance and tilt noise corrections to ambient noise data recorded by OBSs. Waves faster than the fundamental mode Rayleigh/Scholte waves are retrieved after noise correction. These waves must couple better with the solid earth than water because they are retrieved after compliance removal. They are likely a mix of more than one higher modes of Rayleigh/Scholte 
waves.

4. Gives a reference to phase unwrapping at short periods. As the inter-station distances are short $(2-10 \mathrm{~km})$, the dispersion curves are mainly at short periods $(<6.5 \mathrm{~s})$. In this situation, cycle skipping during phase velocity measurement is a challenge because dispersion curves of different cycles at short periods are very close to each other. Several reference models are made to unwrap the phase, including associated phase velocities which count all station pairs, and a forward-modeled dispersion curve using a model in an adjacent area.

5. Indicates from the dispersion curves and the inverted shear velocity models, the velocity in the south of the back-arc rifting center is slower than the velocity in the north, which may be caused by the high heat flow in the south region. The high heat flow may be a consequence of asymmetric back-arc extension and/or rifting.

6. Suggests that there is likely a cooled magma chamber below a small volcanic outcrop in the deployment region. 


\section{Chapter 6}

\section{Conclusions}

In this thesis, we analyse some ocean bottom seismometer data from the Hikurangi subduction zone offshore New Zealand and the southwestern Okinawa trough offshore Taiwan to study the slow slip events and back-arc tectonics, respectively. In this chapter, we answer the questions posed in Chapter 1 and outline the key contributions to our understanding of these two subduction zones.

1. What is the best practice for processing ocean bottom seismometer ambient noise data?

The main dataset used in this thesis have been collected using Ocean Bottom Seismometers (OBSs). OBSs provide us direct observations above the subduction zones. Here we suggest the best practise of processing OBS data for reference in future studies. Processing OBS data is generally more difficult when compared with on-land seismometer data because of how the OBSs are deployed, effects from ocean wave noise, and instrument timing. 1) Deployment. When deploying an OBS, it is dropped from a ship and allowed to freely fall to the ocean bottom with unknown conditions, in contrast to burying a seismograph onshore after levelling and orienting it. Therefore, the orientation of OBSs is unknown, and the horizontal level is not guaranteed. Although some types of seismometers are capable of levelling and orienting themselves after arriving at the seafloor. Nevertheless, the seismometers can still be tilted by the water waves. 2) Ocean wave noise. As OBSs stay on the seafloor, they can be affected by the ocean waves around them. Chapter 5 introduces seafloor compliance noise and tilt noise, as well as the cur- 
rent techniques to correct these two types of noise. In this thesis, we find such correction is not helpful in improving short period data quality. However, it may be applied to retrieve high mode surface waves. Therefore, the correction is not applied in Chapters 3 and 4. 3) Timing. OBSs are not equipped with GPS clocks and their clocks are affected by pressure and temperature variations under the sea. Therefore, the timing of OBSs is not very accurate. Velocity variations studied in Chapters 3 and 4 have a high sensitivity to timing. Therefore, the main results are computed using only single OBS stations. Timing does not affect single station cross-correlations because different channels at one OBS station use the same clock.

2. How does the occurrence of slow slip events influence seismic velocities? Are velocity variations associated with the occurrence of slow slip events in New Zealand detectable?

In Chapters 3 and 4, we study seismic velocity variations related to slow slip events (SSEs) in the northern Hikurangi Margin offshore Gisborne. Chapter 3 uses continuous ambient noise data recorded by 15 OBSs of the Hikurangi Ocean Bottom Investigation of Tremor and Slow Slip II (HOBITSS II) deployment during 20142015. During the deployment, four main SSEs occurred, from which the biggest one, SSE2, occurred right underneath the deployment with a slip larger than 250 $\mathrm{mm}$. Chapter 4 uses continuous ambient noise data recorded by 5 OBSs deployed in 2018-2019 by the HOBITSS V. One SSE occurred during the deployment. We calculate single station cross-component correlations and use the coda waves to compute velocity variations. Our results suggest that velocity variations associated with the occurrence of SSEs in New Zealand are detectable. We find that the velocity decreases during the SSEs and increases after the SSEs. There are also possible velocity increases before the SSEs. While the velocity variations during 2014-2015 occur at 2.5-14 s, the velocity variations during 2018-2019 occur at 1-2.5 s, suggesting the velocity variations during the 2018-2019 SSE were at shallower depths compared to the velocity variations during the 2014-2015 SSE.

3. What are the physical processes by which slow slip events cause velocity variations?

Combining the findings from Chapters 3 and 4, we suggest: 
1) There are two potential physical processes that result in velocity variations due to SSEs: a) Fluid migration related to fault-valve behavior between the two plates: Before the SSE, there is a permeability seal on the plate boundary that traps fluid beneath the subduction interface and fluid pressure steadily increases, to the point at which the SSE is triggered (Warren-Smith et al., 2019). The SSE breaks the seal on the plate boundary, and the trapped fluids migrate to the upper plate. We observe a velocity decrease in the upper plate during the SSE that is consistent with the increased fluid volume in the upper plate. After the SSE, the plate boundary re-seals, the fluids in the upper plate diffuse, and velocity increases again. This interpretation is consistent with that of Warren-Smith et al. (2019) and Zal et al. (2020) for changes in stress ratio and Vp/Vs respectively. b) Crustal strain changes through the SSE cycle: Before the SSE, the plates are locked together along the plate interface, and elastic strain (largely contraction) accrues in the overriding plate. The occurrence of the SSE relieves the accumulated elastic strain, resulting in dilation and an increase of porosity. We observe a velocity decrease in the upper plate during the SSE, which may be due to the increased porosity. A porosity increase can lead to an increase in fluid volume. After the SSE, the plate boundary re-locks, and contraction begins again, resulting in a subsequent porosity decrease and velocity increase.

2) We point out that aspects of both interpretations may be at play to explain the observed seismic velocity changes. Both of them can possibly lead to fluid volume changes, but the volume and pathway of the fluids are unconstrained. A combination of fluid migration related to fault-valve behavior and consequences of crustal strain during the SSE may explain the velocity variations observed in this thesis and previous research (Warren-Smith et al., 2019; Zal et al., 2020; Shaddox and Schwartz, 2019; Todd et al., 2018). However, only elastic strain changes can explain the velocity increases before the SSEs (if they are real) and fluid migration cannot explain them.

4. What is the seismic velocity structure in the southwestern Okinawa Trough? How does it relate to the regional tectonics?

In Chapter 5, we study the shear wave velocity structure in the southwestern Okinawa Trough offshore northeastern Taiwan. The Okinawa Trough is a backarc basin in the process of opening. We use the continuous ambient noise data 
recorded by 34 OBSs deployed at various times from 2010 to 2018. We compute cross-station correlations using seismic vertical channels and pressure gauges. We measure phase velocities from retrieved Rayleigh waves. We invert for three 1D shear velocity models in three sub-regions (south and north of the rifting axis and northwest edge of the study region) based on the phase velocities of Rayleigh/Scholte waves. We find that the phase and shear velocities in the south of the rifting axis and the northwest edge of the study region are slower than that in the north of the rifting axis. The velocity difference suggests the velocity structure in the southwestern Okinawa Trough is asymmetric along the rifting axis. Previous studies have shown high heat flows (about $110 \mathrm{~mW} / \mathrm{m}^{2}$ on average) in the south of the rifting axis. The low velocity in the south could be caused by the high heat flow in this region which may be caused by asymmetric back-arc extension and rifting. We acknowledge that we do not have many constraints in the south due to the limited station coverage in the south region. This study provides shear velocity models in the southwestern Okinawa Trough and information on the back-arc tectonics.

The key contributions of this thesis and suggested future work include:

1. This thesis focuses on OBS continuous data, providing effective methods and examples of OBS data processing for ambient noise applications.

2. This thesis studies temporal velocity variations related to SSEs, providing evidence to support some hypotheses of the SSE mechanism. Further networks of ocean instruments and studies are still required to improve our understanding of SSEs and subduction.

3. This thesis studies shear velocity structure in the southwestern Okinawa Trough, providing evidence for the back-arc extension and rifting. In the future, we will conduct a 3D shear velocity model to provide a better resolution of the shear velocity structure in the study region.

Future work:

1. A precise calculation for strain following Rivet et al. (2011) will be conducted, to give a better estimation for porosity changes through the SSE cycle, and to study its relation to velocity variations. 
2. More OBSs have been deployed in the northern Hikurangi. After the data are collected, velocity variations will be calculated to test the hypotheses proposed in this thesis. A focus will be on testing if there are velocity increases before SSEs, which are observed but not obvious from this thesis.

3. In the southwestern Okinawa Trough, while the $1 \mathrm{D}$ inversion in this thesis proposes an averaged shear wave velocity structure in the study region, the lateral velocity changes in the study region remain unknown. This knowledge which would help constraint whether the shear velocity in the center region near the rifting axis is different from the shear velocity far away from the rifting center. Traditional 2D tomography at different depths loses lateral spatial correlations during the inversion and introduces more uncertainty. Future work for a 3D shear velocity model will be conducted to constrain a 3D shear wave velocity structure in the southwestern Okinawa trough to provide a more detailed shear velocity model (e.g., lateral shear velocity variations in the north of the rifting center). Funding from the HPC-Europa3 project was granted in 2020, providing computing resources from the Edinburgh Parallel Computing Centre, University of Edinburgh. Working with Andrew Curtis, we will apply 3D Markov Chain Monte Carlo to the dispersion curves measured in this chapter. This 3D inversion will constrain a 3D shear wave velocity structure in the southwestern Okinawa trough to provide more details of the structure, helping to better understand the extension and rifting of the back-arc. Due to the travel restriction because of the Covid-19, the collaboration will be transferred online. 


\section{Appendix A}

\section{Appendix A: Chapter 3}

This chapter provides appendix material for Chapter 3: Temporal velocity variations in the northern Hikurangi margin and the relation to slow slip.

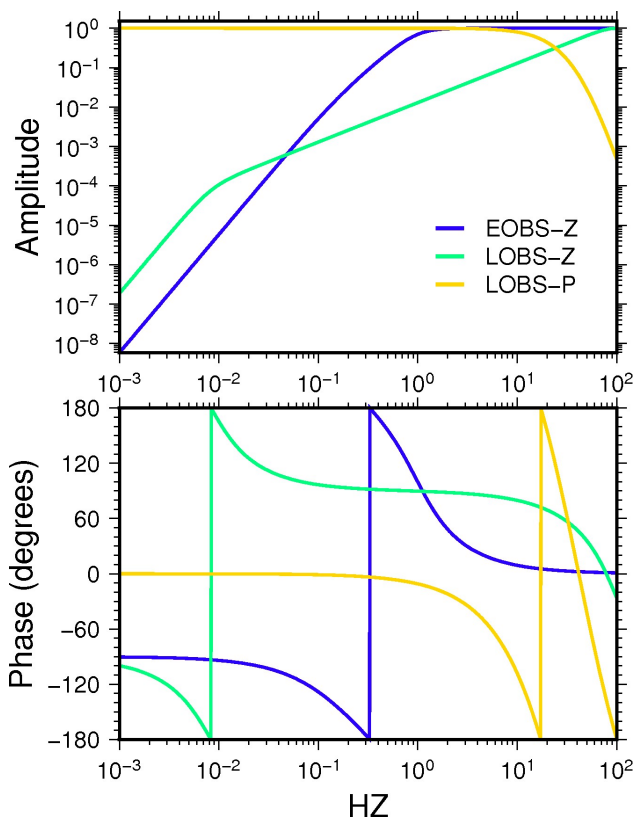

Figure A.1: Instrument responses of vertical seismometers $(\mathrm{Z})$ and pressure gauges $(\mathrm{P})$ of different types of instruments deployed in the Hikurangi margin, with amplitude normalized to 1 . The instrument responses of horizontal components are the same as the vertical components $(Z)$. 
Table A.1: Locations of the ocean bottom seismometers deployed by the HOBITSS II.

\begin{tabular}{lccc}
\hline Station & Longitude & Latitude & Altitude $(\mathrm{m})$ \\
\hline LOBS1 & 178.818700 & -38.592100 & -993.6 \\
LOBS2 & 179.046100 & -38.621000 & -3562.5 \\
LOBS3 & 179.147300 & -38.792200 & -3540.3 \\
LOBS4 & 178.981500 & -39.120100 & -3440.5 \\
LOBS5 & 178.698400 & -39.119300 & -2361 \\
LOBS6 & 178.796000 & -38.977800 & -1873.5 \\
LOBS7 & 178.568100 & -38.711500 & -784.1 \\
LOBS8 & 178.459400 & -38.843200 & -651.3 \\
LOBS9 & 178.521400 & -39.071600 & -1457.4 \\
LOBS10 & 178.313200 & -39.133300 & -1444 \\
EOBS1 & 178.678900 & -38.745900 & -995 \\
EOBS2 & 178.583500 & -38.777100 & -930 \\
EOBS3 & 178.650600 & -38.694600 & -1023 \\
EOBS4 & 178.819900 & -38.688800 & -1712 \\
EOBS5 & 178.325700 & -38.994400 & -1348 \\
\hline
\end{tabular}

Table A.2: Velocity (Yarce et al., 2019) and density (Eberhart-Phillips et al., 2020) models used to compute the sensitivity kernels for the HOBITSS II region. The depth here denotes the bottom depth of the layer beneath sea level. The water depth is about $1.38 \mathrm{~km}$ in the study region.

\begin{tabular}{lccc}
\hline Depth $(\mathrm{km})$ & $\mathrm{Vp}(\mathrm{km} / \mathrm{s})$ & $\mathrm{Vs}(\mathrm{km} / \mathrm{s})$ & Density $\left(\mathrm{g} / \mathrm{cm}^{3}\right)$ \\
\hline 1.38 & 1.5 & 0 & 1.03 \\
7.38 & 2.496 & 1.269 & 2.348 \\
12.38 & 4.081 & 2.494 & 2.551 \\
16.38 & 5.929 & 3.618 & 2.980 \\
23.38 & 6.548 & 3.637 & 3.191 \\
51.38 & 8.481 & 4.797 & 3.441 \\
\hline
\end{tabular}




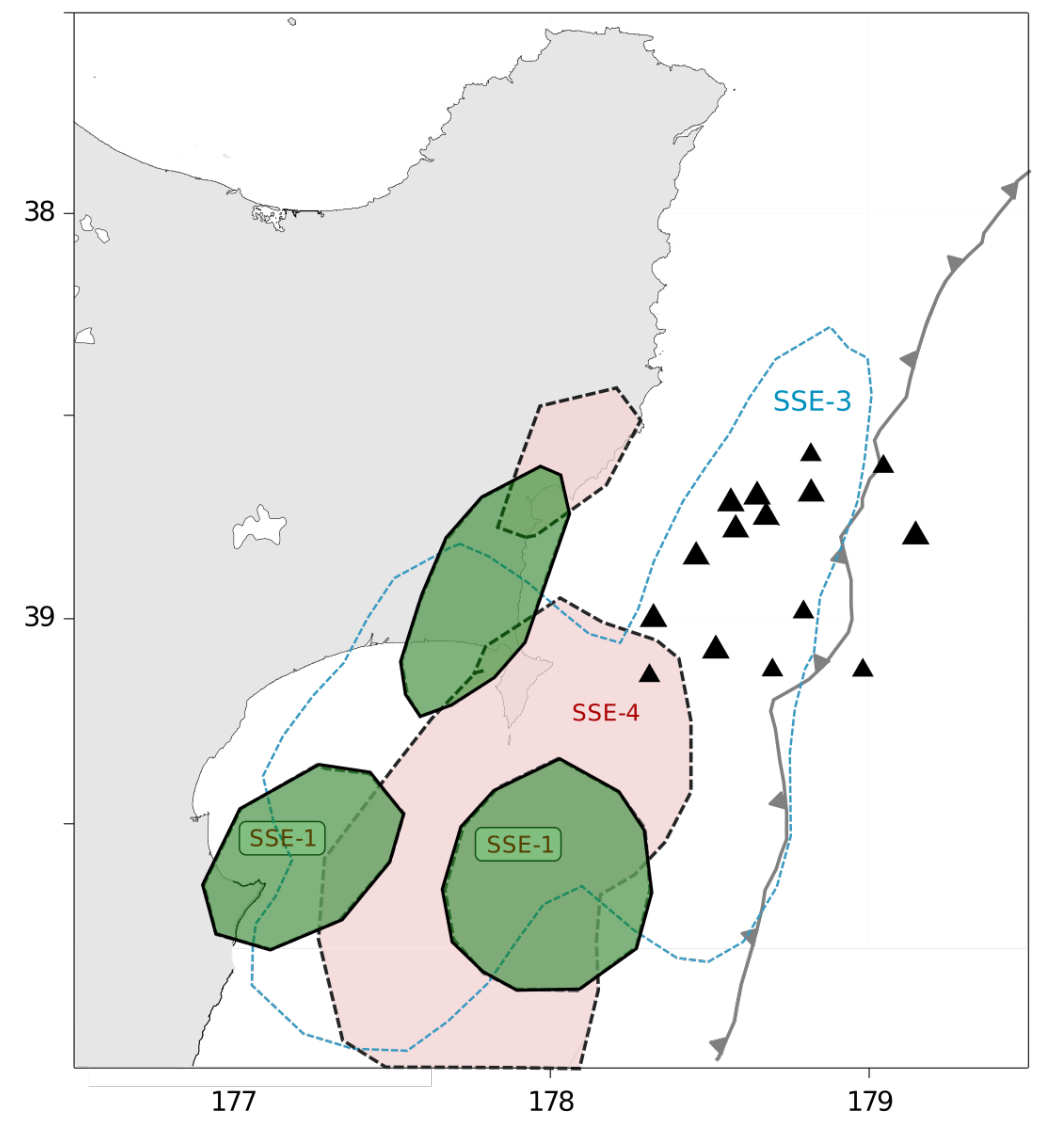

Figure A.2: Locations of SSE1, SSE3 and, SSE4 (Warren-Smith et al., 2019). The green shades mark the regions where SSE1 occurred, the blue dashed line outlines SSE3, the pink shades mark the location of SSE4. 


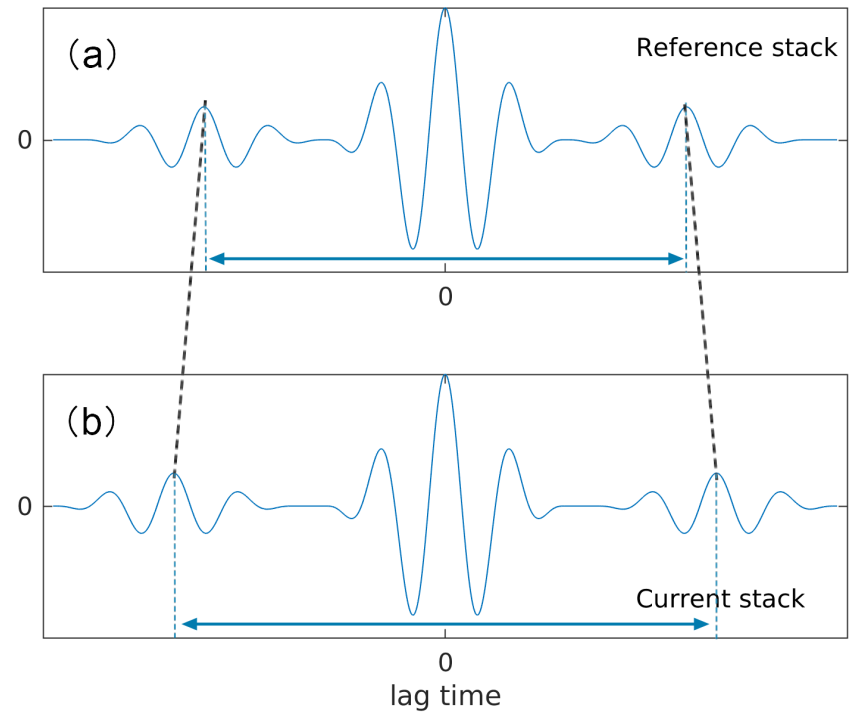

Figure A.3: Velocity variation measurement (schematic). The waves at 0 lag time are direct arrivals. The coda denoted by the dashed lines at both positive and negative lags are scattered waves. The blue arrows indicate the arrival time of the coda waves. (a) Reference stack. (b) Current stack of the same station after a velocity change. There is a travel time difference between the reference and current stack. The relative velocity change $\mathrm{dv} / \mathrm{v}$ can be determined by $-\mathrm{dt} / \mathrm{t}$. 


\section{A.1 Parameterization}

Parameters in MSNoise are tested before they are determined. Different filters are tested and compared using lag-time dependent SNR (Larose et al., 2007; Clarke et al., 2011)

$$
\operatorname{SNR}(N, t)=\frac{s(N, t)}{\sigma(N, t)}
$$

where $\mathrm{N}$ is the stack number of individual days, $\mathrm{t}$ is lag time, $s(N, t)$ is the signal level, and $\sigma(N, t)$ is the noise level.

The signal level $s(N, t)$ is the Hilbert envelope of an average of single-day crosscorrelations

$$
s(N, t)=|<c c(t)>+i H(<c c(t)>)|
$$

where $<.>$ is the average over $\mathrm{N}$ single functions $\mathrm{cc}(\mathrm{t})$ and the Hilbert transform $\mathrm{H}($.$) .$

The noise $\sigma(N, t)$ is computed by measuring the variation of single-day crosscorrelations at each lag time

$$
\sigma(N, t)=\sqrt{\frac{<c c(t)^{2}>-<c c(t)>^{2}}{N-1}} .
$$

Figure A.4 shows an example of LOBS8 vertical-parallel component correlations, shown with its waveform for a better understanding of the lag-time dependent SNR. Other components and other stations have the same features that the filters of 2.5-6 s and 2.5-20 s have high SNR for the coda. Segmentation length (raw data window length to correlate), segmentation overlap (overlap in window length between segments), normalization (1-bit, or windsorizing at $\mathrm{N}$ time RootMean-Square) are also determined by lag-time dependent SNR. Figure A.5 shows examples for how segmentation length and overlap are determined.

Current stack size is determined by correlation coefficients between current stacks and the reference stack. A large size of the current stack can decrease the temporal resolution and might hide some velocity changes happening in a short time, but too small a size of the stack might not be enough to suppress noise. Correlation coefficients between different size current stacks and the reference stack are computed and compared (Figure A.6). Before stacking for both current stacks and the reference stack, the single-day stacks with correlation coefficients smaller than a 
threshold are taken out to exclude noisy data. The threshold varies from station to station.

The coda window on the cross-correlations to compute velocity variations is determined by lag-time dependent SNR (Figure A.9), with the SNR of all the single stations larger than 2. The SNR threshold follows previous studies (Yates, 2018; Yates et al., 2019). MSNoise selects data by coherence. The coherence threshold is determined by the relationship between SNR and coherence to exclude the data with SNR $<2$. The coda window on the negative lag is set to be $-70 \mathrm{~s}$ according to the SNR. The window on positive lag is set to be the same to keep it symmetric. The direct arrivals (-20 s to $20 \mathrm{~s}$ ) are excluded. 
Table A.3: Final parameters used for MSNoise 1.5. L: LOBSs; E: EOBSs.

\begin{tabular}{|c|c|c|}
\hline Parameter & Description & Value \\
\hline startdate & start date for computation & 2014-05-15 \\
\hline enddate & end date for computation & 2015-06-23 \\
\hline ref_begin & start date of reference stack & 2014-05-15 \\
\hline ref_end & end date of reference stack & $2015-06-23$ \\
\hline maxlag & maximum lag of cross-correlations & 300 \\
\hline cc_sampling_rate & sampling rate for the cross-correlation & 20 \\
\hline preprocess_lowpass & preprocessing low-pass filter & $2.0 \mathrm{~Hz}$ \\
\hline preprocess_highpass & preprocessing high-pass filter & $0.02 \mathrm{~Hz}$ \\
\hline remove_response & remove instrument response & $\mathrm{N}$ \\
\hline corr_duration & data windows to correlate & 14400s (L), 1800s (E) \\
\hline overlap & overlap between data windows & 0.7 \\
\hline windsorizing & windorizing at $\mathrm{N}$ times $\mathrm{RMS}$ & 3 \\
\hline whitening & whiten Traces before cross-correlation & A (all traces) \\
\hline stack_method & linear or phase weighted stack & linear \\
\hline autocorr & compute single station or not & Y \\
\hline mov_stack & current stack size & 20 days \\
\hline low & the lower frequency bound of whitening & $0.05 \mathrm{~Hz}$ \\
\hline high & the upper frequency bound of whitening & $0.4 \mathrm{~Hz}$ \\
\hline mwcs_low & the lower frequency bound for MWCS & $0.07 \mathrm{~Hz}$ \\
\hline mwcs_high & the upper frequency bound for MWCS & $0.4 \mathrm{~Hz}$ \\
\hline mwcs_wlen & window length to perform MWCS & $20 \mathrm{~s}$ \\
\hline mwcs_step & step of the moving window in MWCS & $4 \mathrm{~s}$ \\
\hline dtt_lag & how the window is defined & static \\
\hline dtt_minlag & min lag time to compute $\mathrm{dtt}$ & $0 \mathrm{~s}$ \\
\hline dtt_width & window length to compute $\mathrm{dtt}$ & $70 \mathrm{~s}$ \\
\hline dtt_sides & which sides of stacks to use & both \\
\hline dtt_mincoh & threshold of coherence for data & 0.89 \\
\hline dtt_maxdt & maximum dt measurement & $0.2 \mathrm{~s}$ \\
\hline dtt_maxerr & maximum error on $\mathrm{dt}$ measurement & $0.1 \mathrm{~s}$ \\
\hline
\end{tabular}




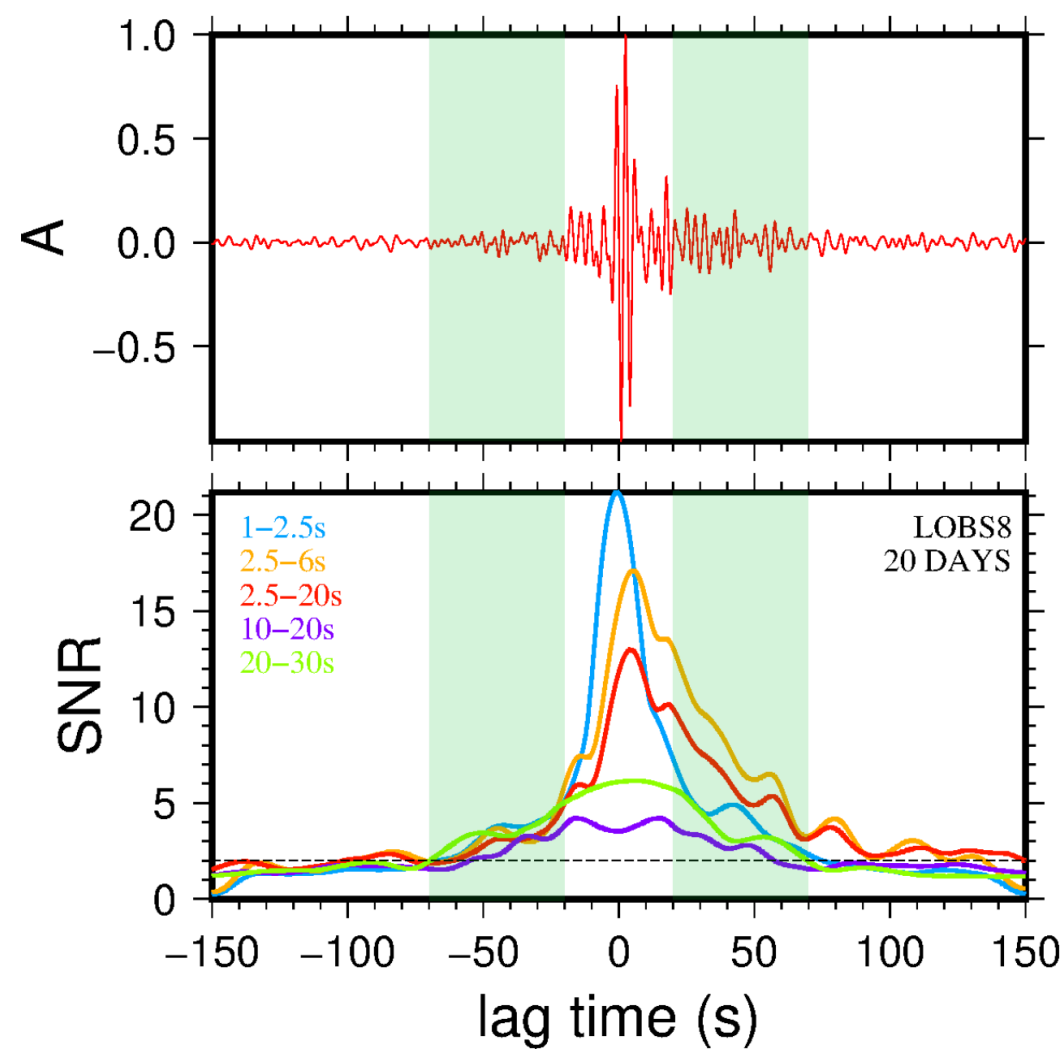

Figure A.4: Different filter comparison on LOBS8 vertical-parallel component correlations. A waveform of 20-day stack filtering at 2.5-20 s is shown on the top, which has a stronger signal on the positive lag. The amplitude is normalized. Lag-time dependent SNR of different filtered 20-day stacks are computed (the bottom figure). The green shades mark the coda wave windows to compute $\mathrm{dv} / \mathrm{v}$, and the horizontal dash line in the bottom figure marks $\mathrm{SNR}=2$. 

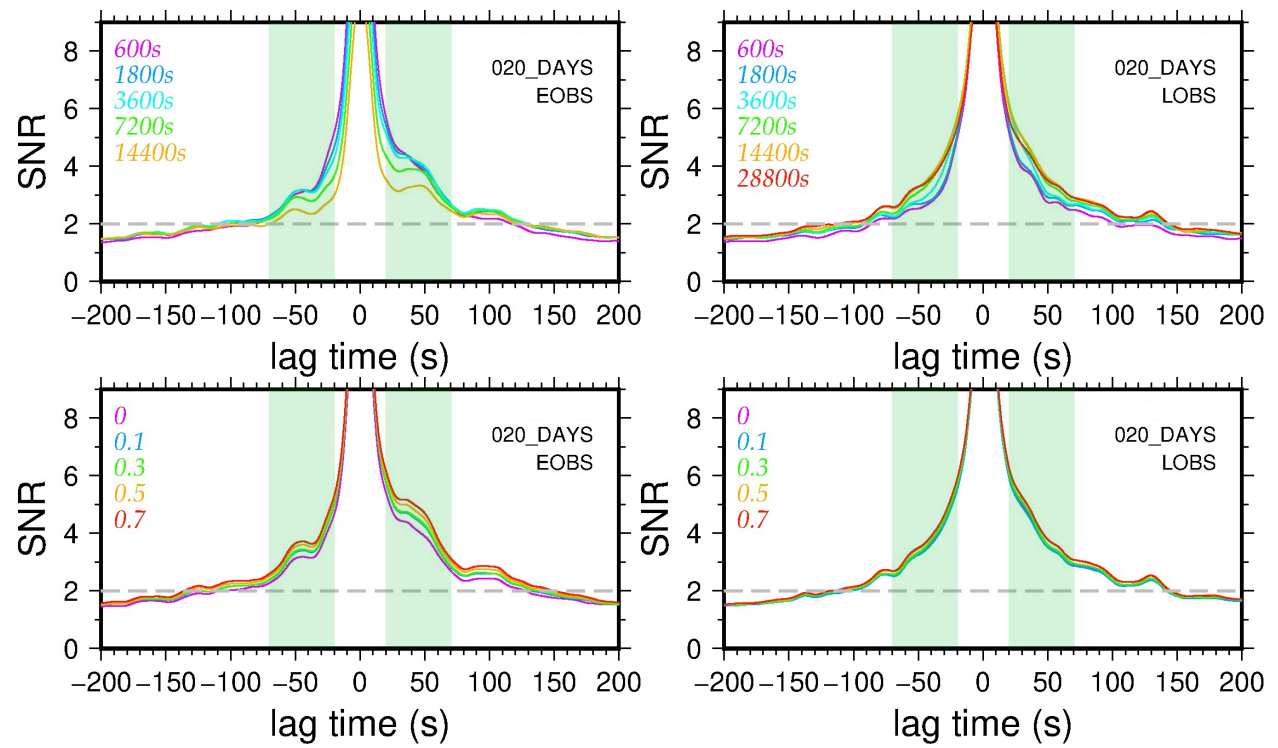

Figure A.5: Lag-time dependent SNR of different segmentation lengths (upper) and segmentation overlaps (bottom). The EOBS SNR (left) are averaged from different components of the five EOBSs. The LOBS SNR (right) are averaged from different components of the four used LOBSs. The green shades mark the coda wave windows to compute $\mathrm{dv} / \mathrm{v}$.

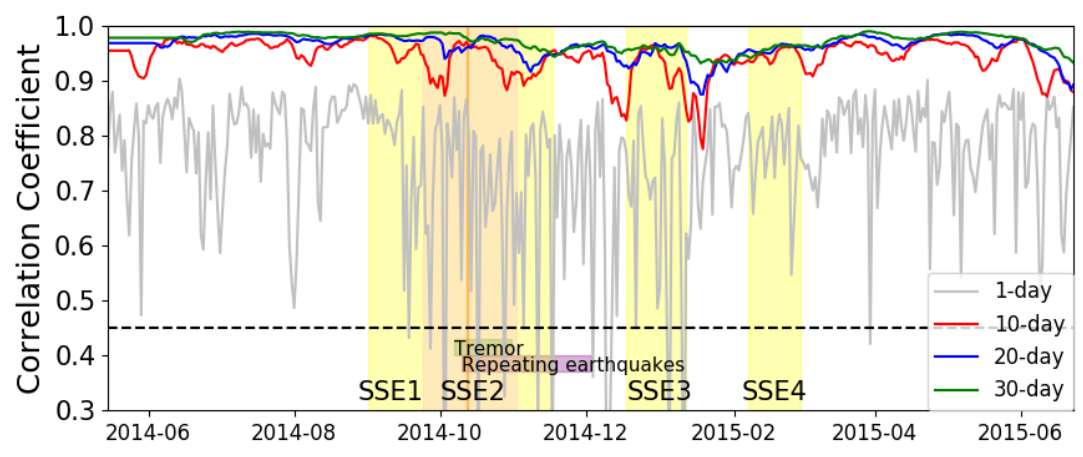

Figure A.6: An example of the relationship between stack sizes and correlation coefficients computed on LOBS8 vertical-parallel component correlations. The occurrence of SSEs, tremors and repeating earthquakes are marked. The vertical orange line in SSE2 marks the time when SSE2 finishes its main slip. Correlation coefficients between different size current stacks (1-day stacks, 10-day stacks, 20-day stacks, and 30-day stacks) and reference stack are computed and compared. From 20-day stacks to 30-day stacks, the correlation coefficient is not improved much. The horizontal black dashed line marks the threshold of correlation coefficient $=0.45$ to exclude daily crosscorrelations for LOBS8 vertical-parallel component correlations. The correlation coefficient thresholds for other stations are, EOBS1: 0.65; EOBS2: 0.55; EOBS3: 0.55; EOBS4: 0.5; EOBS5: 0.3 for parallel-perpendicular cross-correlations, 0.6 for the other two components; LOBS3: 0.1; LOBS7: 0.45; LOBS9: 0.4 for all the three components. 


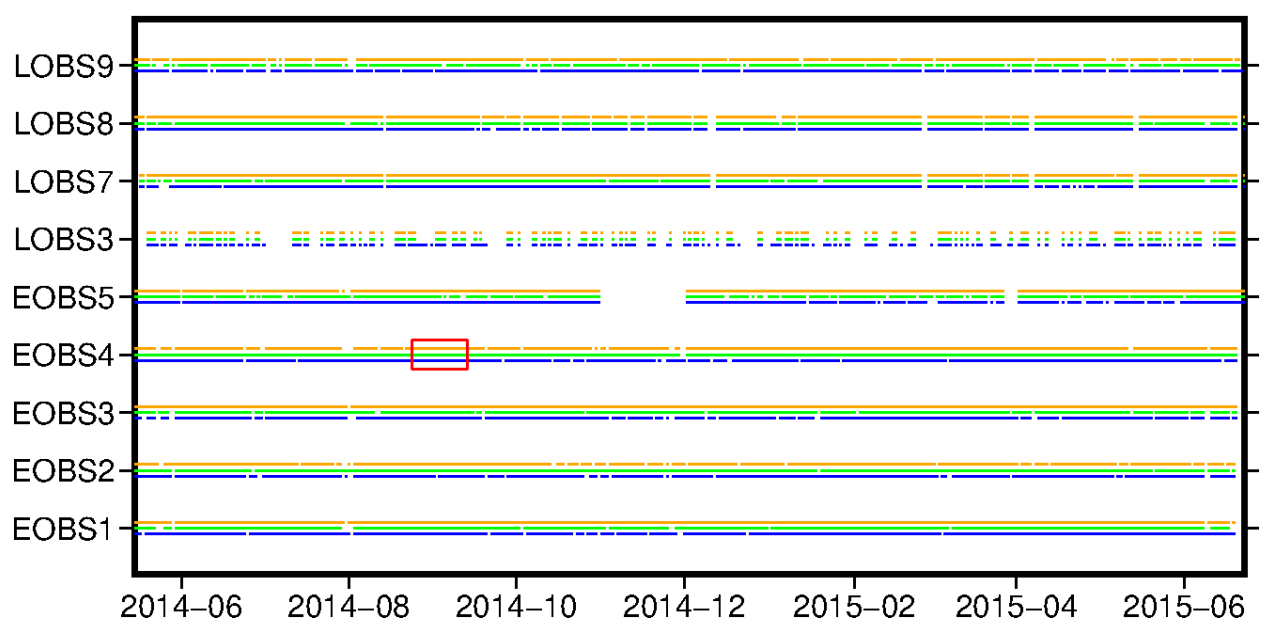

Figure A.7: Available cross-component correlations of used stations after selecting daily crosscorrelations according to the correlation coefficient of the daily cross-correlations and the reference stack. Three colors indicate three different component correlations (Yellow: vertical-parallel; Green: vertical-perpendicular; Blue: parallel-perpendicular). The gap in EOBS5 from the end of 2014 to early 2015 is because of the lack of data. LOBS3 also lost some data from different days, and so the $\mathrm{dv} / \mathrm{v}$ result from LOBS3 is less reliable. The red rectangle denotes a 20-day window. The daily cross-correlations within the window are stacked to be a current stack.
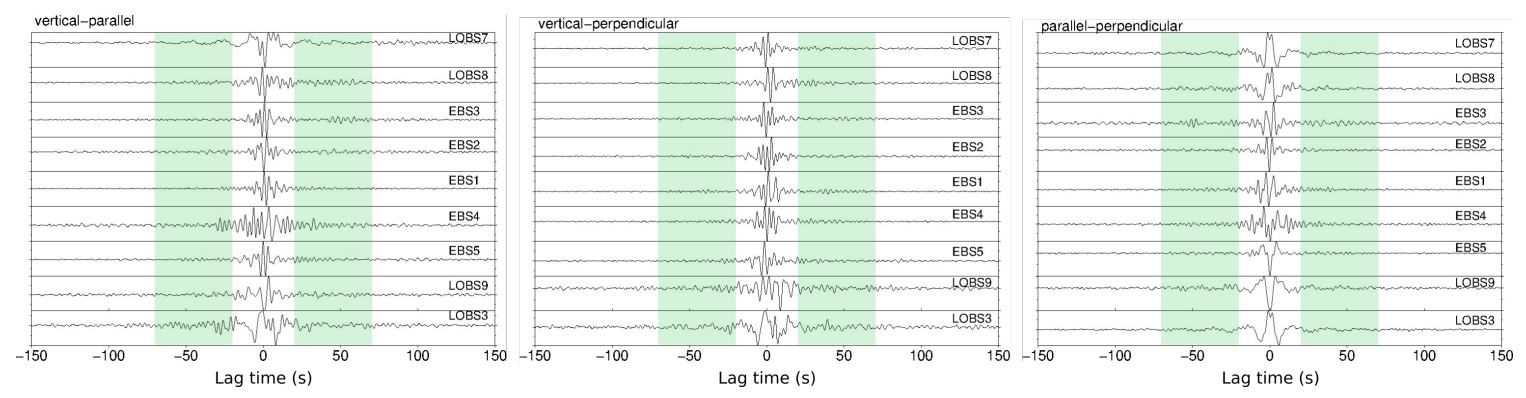

Figure A.8: Reference stacks on the three components, plotting from the closest (top) to the furthest (bottom) distance from the coast. The green shades mark the coda wave windows to compute $\mathrm{dv} / \mathrm{v}$. 

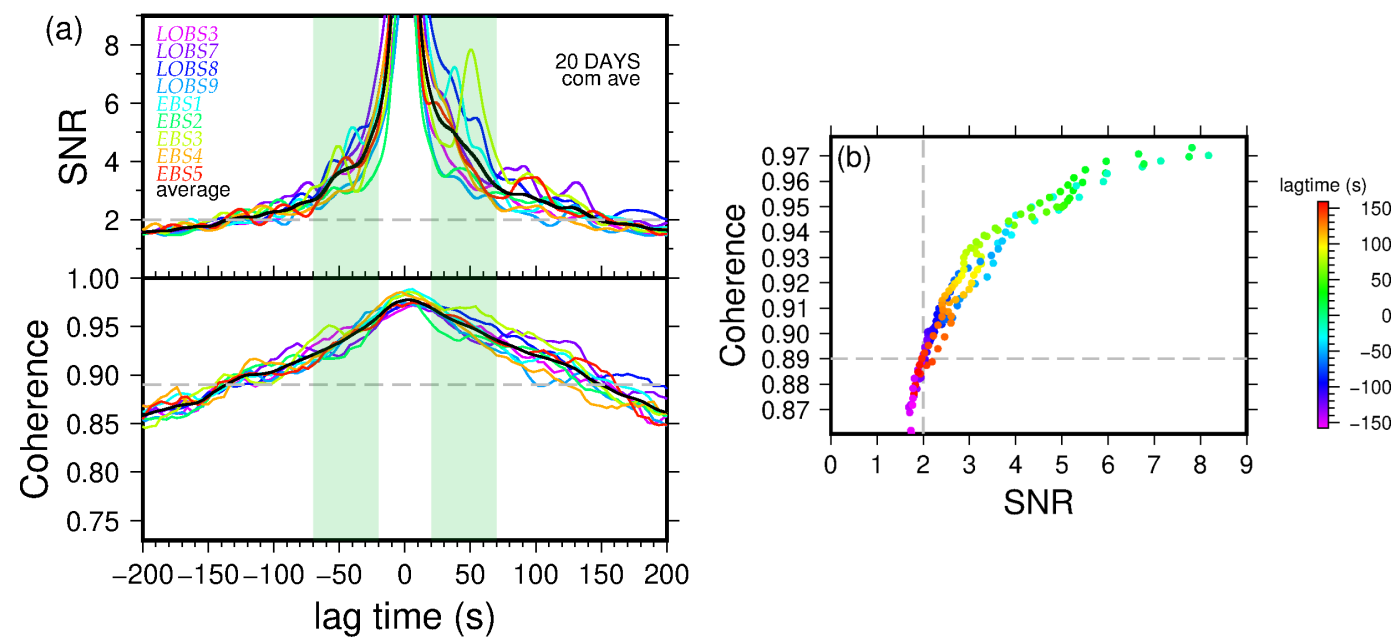

Figure A.9: Determination of coda wave window and threshold of coherence. (a) The final lagtime dependent SNR and the coherence between the 20-day current stacks and the reference stack of single stations (color-coded) and their average (black), computed using determined parameters. For each station, the SNR is averaged from its three components. The SNR of each component is averaged from all the 20-day current stacks. The green shades mark the coda wave windows to compute $\mathrm{dv} / \mathrm{v}$, determined by the SNR of all the single stations above 2 . The horizontal dashed line on the top figure marks the threshold of SNR, and the one on the bottom figure marks the threshold of coherence. (b) Relationship between the average SNR and coherence (black curves in (a)). Different colors denote the values at different lag times. The threshold of coherence (0.89) is determined by SNR $>2$. The dashed lines mark the thresholds of SNR and coherence.

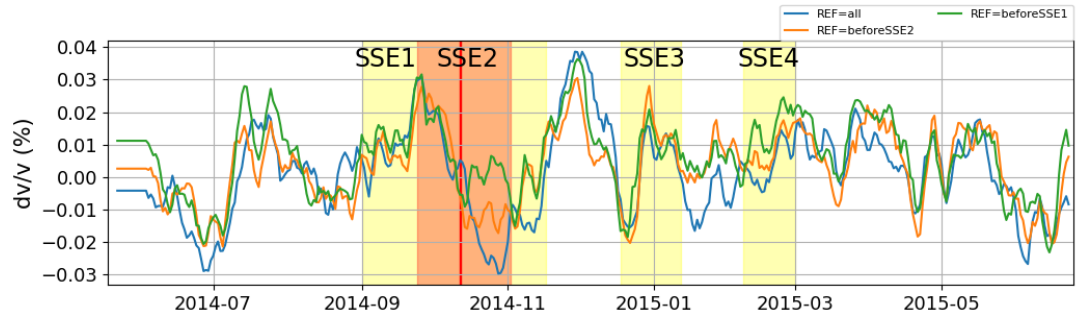

Figure A.10: Comparison of velocity variations using a reference stack of all days (blue), the days before SSE1 (green), and the days before SSE2 (orange). The four SSEs are marked, and the red lines mark when SSE2 finishes its main slip.

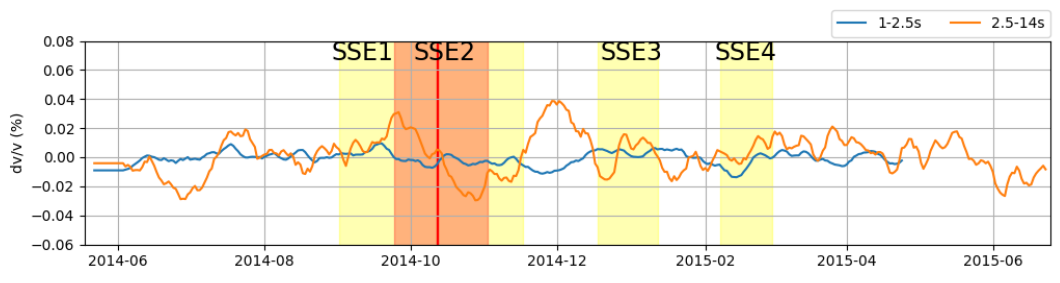

Figure A.11: Comparison of velocity variations computed at 1-2.5 s (blue) and 2.5-14 s (orange), using 20-day moving window stacks. 


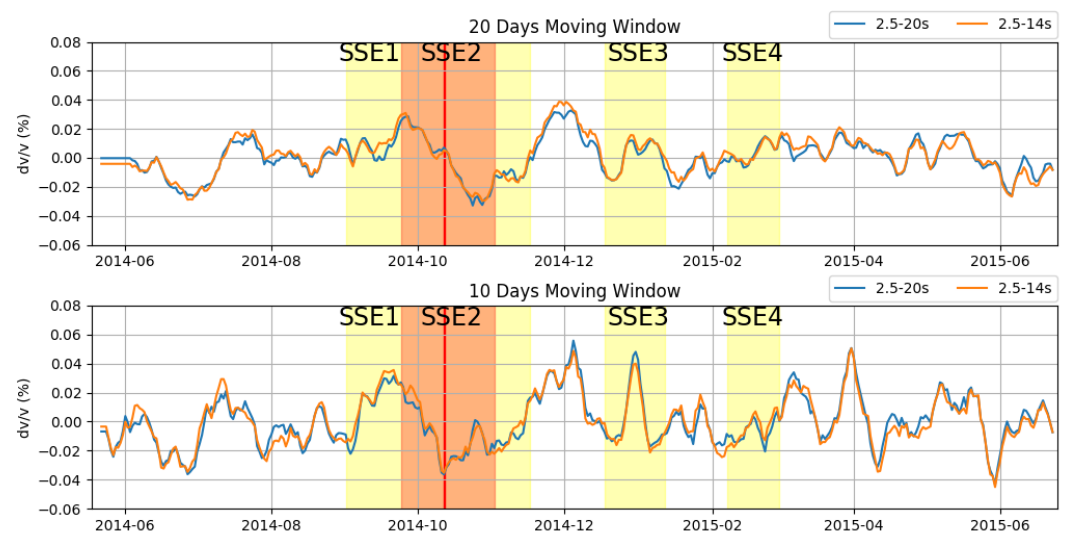

Figure A.12: Comparison of velocity variations computed at 2.5-20 s (blue) and 2.5-14 s (orange), using 20-day moving window stacks and 10-day moving window stacks. The results at 2.5-20 $\mathrm{s}$ are similar to the results at 2.5-14 s. The velocity decreases caused by SSE2 happen from 24th September to 28th October 2014 in the 20-day moving window result and 20th September to 12th October 2014 in the 10-day moving window result. This time difference is because of the time shift from different stack sizes. Although the timing of SSE2 on the 10-day moving window dv/v terminates earlier than that on 20-day moving window dv/v, the velocity decrease on 10-day moving window $\mathrm{dv} / \mathrm{v}$ happens in the main slip period of SSE2. After the main slip of SSE2, velocity may either increase or decrease.

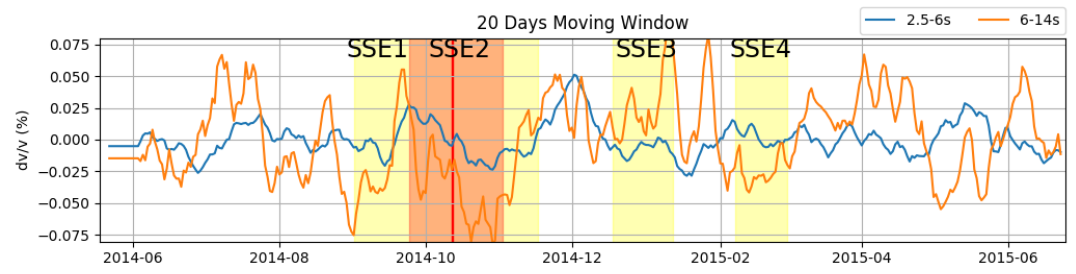

Figure A.13: Comparison of velocity variations computed at 2.5-6 s (blue) and 6-14 s (orange), using 20-day moving window stacks. The results at 6-14 s are noisy but still show a velocity decrease during SSE2 and a velocity increase after it.

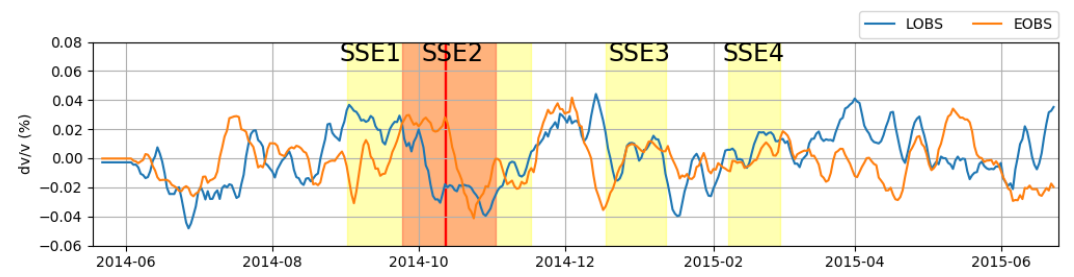

Figure A.14: Comparison of velocity variations computed by LOBSs (blue) and EOBSs (orange) single station cross-component correlations. 


\section{A.2 Seawater pressure effects}

The velocity variations smoothed by the 2-day window are compared to pressure variations (Figure A.15) to test if some variations are related to the seawater pressure changes. Six stations, LOBS1, LOBS4, LOBS6, LOBS8, LOBS9, and LOBS10, are equipped with absolute pressure gauges. Pressure data recorded by these LOBSs are 2-day lowpass filtered and averaged. Previous studies (e.g., King, 1966) showed shear wave velocities increase with hydrostatic pressure. The seawater column puts hydrostatic pressure on the upper plate. An increase in hydrostatic pressure from the water column change can cause an increase in shear wave velocity. The pressure and dv/v variations are different around SSE2 but are similar before and after SSE2, implying seawater pressure changes might cause variations unrelated to SSE2. There is a velocity increase in July 2014 that is likely to be caused by a seawater pressure change.

The biggest pressure increase in July 2014 is about $7 \mathrm{hPa}$. According to a relationship of shear velocity and effective pressure (Eberhart-Phillips et al., 1989), assuming there is no change on porosity and clay content, shear velocity change caused by pressure change can be written as $\delta V s=0.361\left[\left(P e_{2}-e^{-16.7 P e_{2}}\right)-\left(P e_{1}-\right.\right.$ $\left.\left.e^{-16.7 P e_{1}}\right)\right]$. Here, the effective normal stress difference $P e_{2}-P e_{1}=7 h P a$. Assuming there is no pore fluid pressure change during that time, $7 \mathrm{hPa}$ can cause a shear velocity change of $0.3 \times 10^{-2} \mathrm{~m} / \mathrm{s}$, which is much smaller than the corresponding velocity increase $1380 \times 0.05 \%=0.7 \mathrm{~m} / \mathrm{s}$. Therefore, a change of pressure is too small to cause that velocity variation.

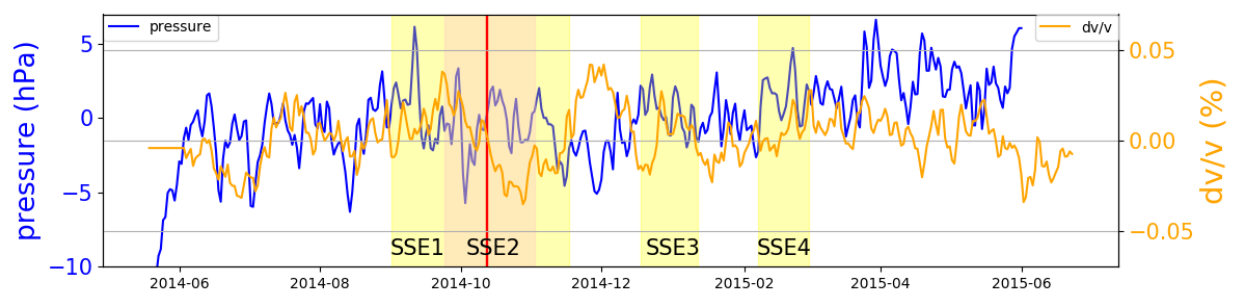

Figure A.15: 20-day moving window velocity variations smoothed by 2-day window compared with 2-day low-pass filtered pressure. 


\section{A.3 Cross-station correlations}

Except for single station cross-component correlations, $\mathrm{dv} / \mathrm{v}$ including cross-station correlations are computed (Figure A.16). Since the timing of OBSs is not accurate because they are not equipped with GPS clocks, the result including cross-station correlations is not as good as that using only single-station correlations. However, the $\mathrm{dv} / \mathrm{v}$ computed, including cross station correlations, still shows a similar decrease and increase during and after SSE2.

Because EOBSs have good data quality and instrument timing, velocity variations are computed using their cross-station correlations, single station cross-component correlations, and auto-correlations (Figure A.17), which also show a velocity decrease during SSE2 and an increase after SSE2 - similar to the result using only single station cross-component correlations in the main text. For comparison, velocity variations are also computed using LOBSs' cross-station correlations, single station cross-component correlations, and auto-correlations (Figure A.18), from which the velocity changes during and after SSE2 are not detected. Therefore, the velocity variations computed from cross-station correlations are only detected by EOBSs. That is might because of the timing issue of LOBSs.

Velocity variations computed using only cross-station correlations from LOBSs and EOBSs (Figure A.19) do not show variations during SSE2, implying the velocity variations during and after SSE2 are mainly detected by single station correlations.

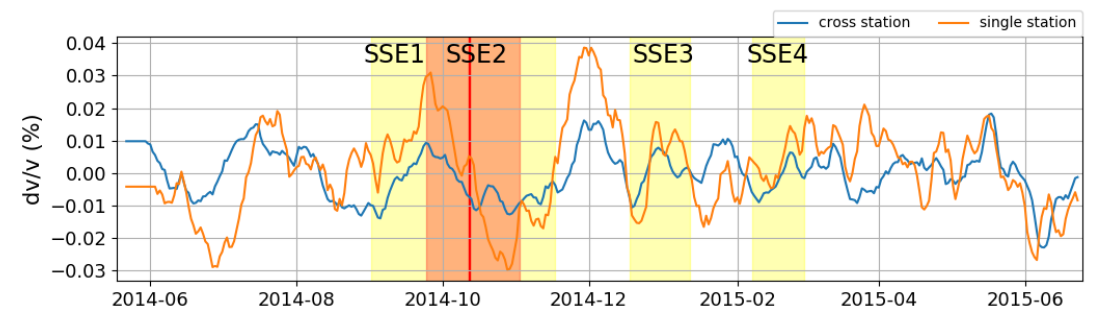

Figure A.16: Comparison to the velocity variations computed including cross station correlations (blue). The orange curve shows the $\mathrm{dv} / \mathrm{v}$ computed using only single station cross-component correlations, which is the result in the main text. The blue curve shows the $\mathrm{dv} / \mathrm{v}$ computed using cross station correlations, single station cross-component correlations, and auto-correlations. The cross station correlations of LOBSs and EOBSs are calculated separately. 


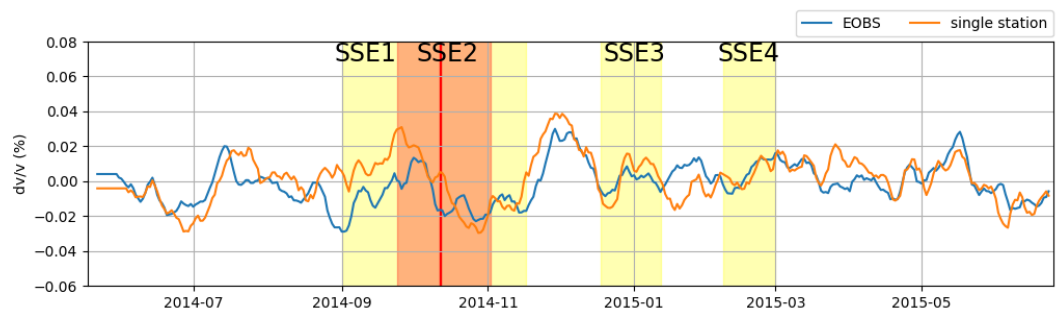

Figure A.17: Comparison to the velocity variations (blue) computed from EOBS cross-station correlations, single station cross-component correlations, and auto-correlations, from nine components: vertical-vertical, parallel-parallel, perpendicular-perpendicular, vertical-parallel, verticalperpendicular, parallel-perpendicular, parallel-vertical, perpendicular-vertical, perpendicularparallel. The orange curve shows the $\mathrm{dv} / \mathrm{v}$ computed using only single station cross-component correlations, which is the result in the main text.

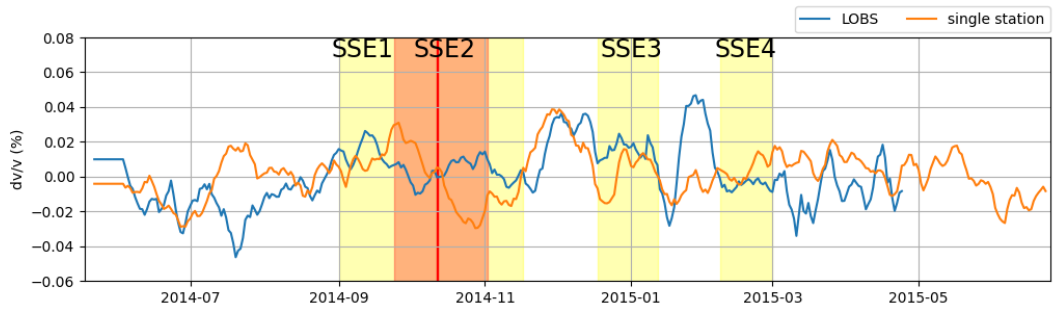

Figure A.18: Comparison to the velocity variations (blue) computed from LOBS cross-station correlations, single station cross-component correlations, and auto-correlations, from nine components: vertical-vertical, parallel-parallel, perpendicular-perpendicular, vertical-parallel, verticalperpendicular, parallel-perpendicular, parallel-vertical, perpendicular-vertical, perpendicularparallel. The result does not show many changes during SSE2. The orange curve shows the $\mathrm{dv} / \mathrm{v}$ computed using only single station cross-component correlations, which is the result in the main text.

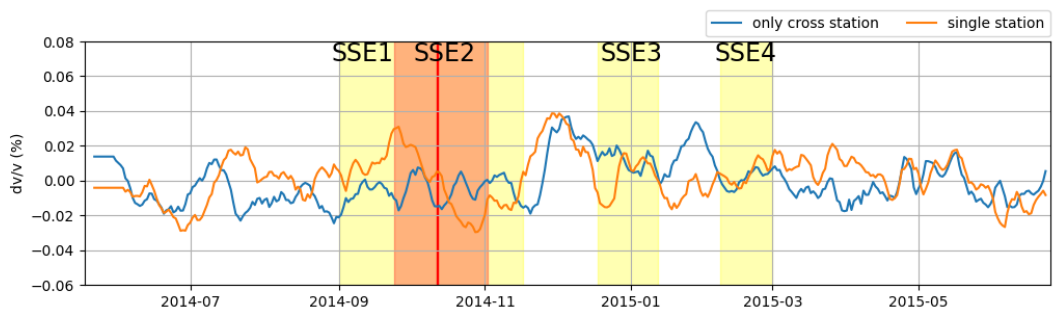

Figure A.19: Comparison to the velocity variations computed using only cross-station correlations from LOBSs and EOBSs. The cross station correlations of LOBSs and EOBSs are calculated separately. 


\section{Appendix B}

\section{Appendix B: Chapter 4}

This chapter provides appendix material for Chapter 4: Temporal velocity variations in 2018-2019 in the northern Hikurangi margin and the relation to slow slip.

Here we investigate if there are any velocity variations related to the earthquakes during the deployment. There are four earthquakes (Figure B.1) near the HOBITSS $\mathrm{V}$ network. The dates of the earthquakes are marked in the 1-2.5 s velocity variation plot (Figure B.2). The velocity does not change after any of the earthquakes.

Velocity variations computed including cross-station correlations are noisy. The velocity variations averaged from different stations (Figures B.3 and B.5) and some examples of velocity variations of individual station-pairs (Figures B.4 and B.6) are shown. Note the average velocity variations (Figures B.3 and B.5) contain

Table B.1: Locations of the ocean bottom seismometers deployed by the HOBITSS V.

\begin{tabular}{cccc} 
Station & Longitude & Latitude & Altitude $(\mathrm{m})$ \\
\hline EOBS1 & 178.8960 & -38.85895 & 2134 \\
EOBS2 & 178.6145 & -38.72728 & 995 \\
EOBS3 & 178.6713 & -38.79293 & 1070 \\
EOBS4 & 178.8382 & -38.71290 & 1989 \\
EOBS5 & 178.7456 & -38.57972 & 910 \\
\hline
\end{tabular}




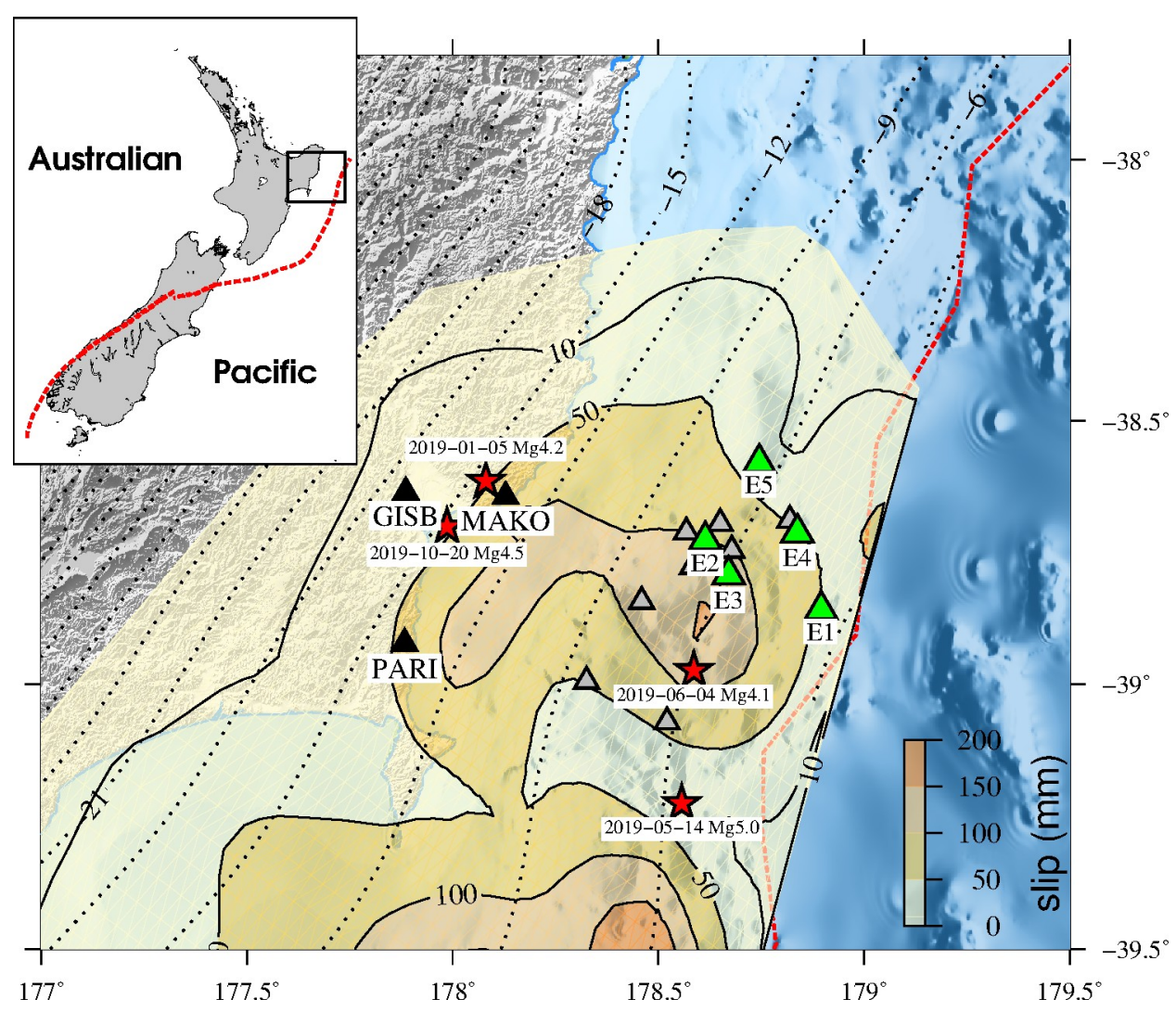

Figure B.1: Red stars mark earthquakes with $\mathrm{Mg}>4.0$ during the HOBITSS V deployment. The dates and magnitudes are written below or above the corresponding stars.

both $\mathrm{dv} / \mathrm{v}$ computed from single stations and cross stations, while the individual station-pair velocity variations (Figures B.4 and B.6) only contain the $\mathrm{dv} / \mathrm{v}$ computed from cross stations. The velocity variations computed including crossstation correlations are not stable. 


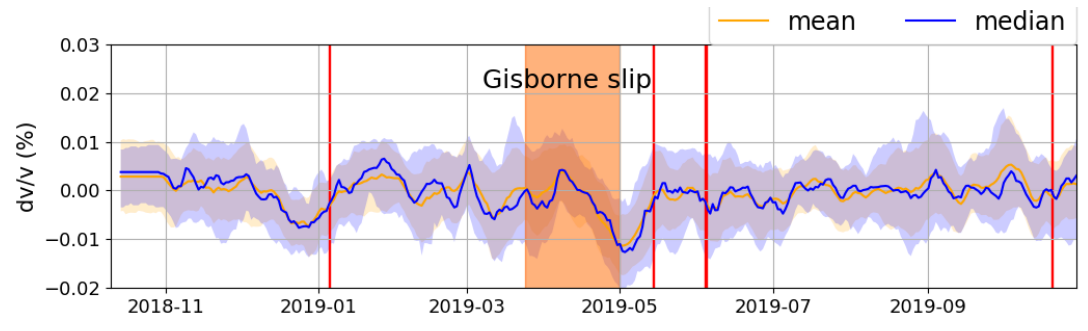

Figure B.2: Single-station-average velocity variations at 1-2.5 s (Figure 4.12). Vertical red lines mark the origin time of the earthquakes.

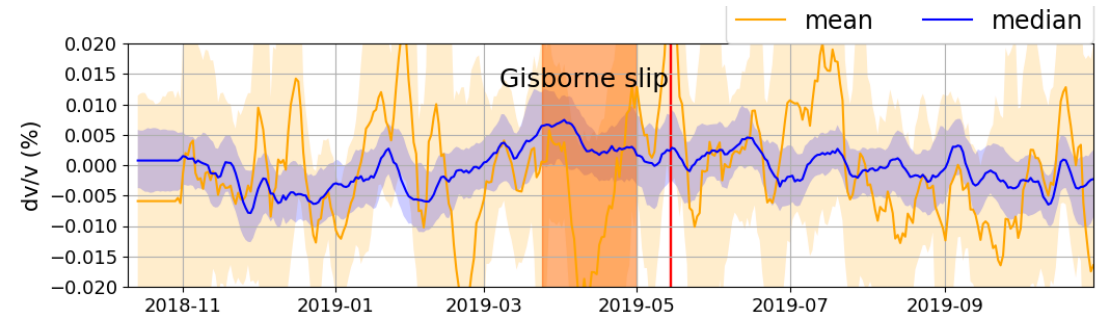

Figure B.3: Velocity variations computed using cross station correlations, single station crosscomponent correlations, and auto-correlations, filtered at 1-2.5 $\mathrm{s}$. The blue line shows the median value from the five OBSs, with the blue shade denotes the errors. The orange line shows the mean value from the five OBSs, with the orange shade denotes the errors. 

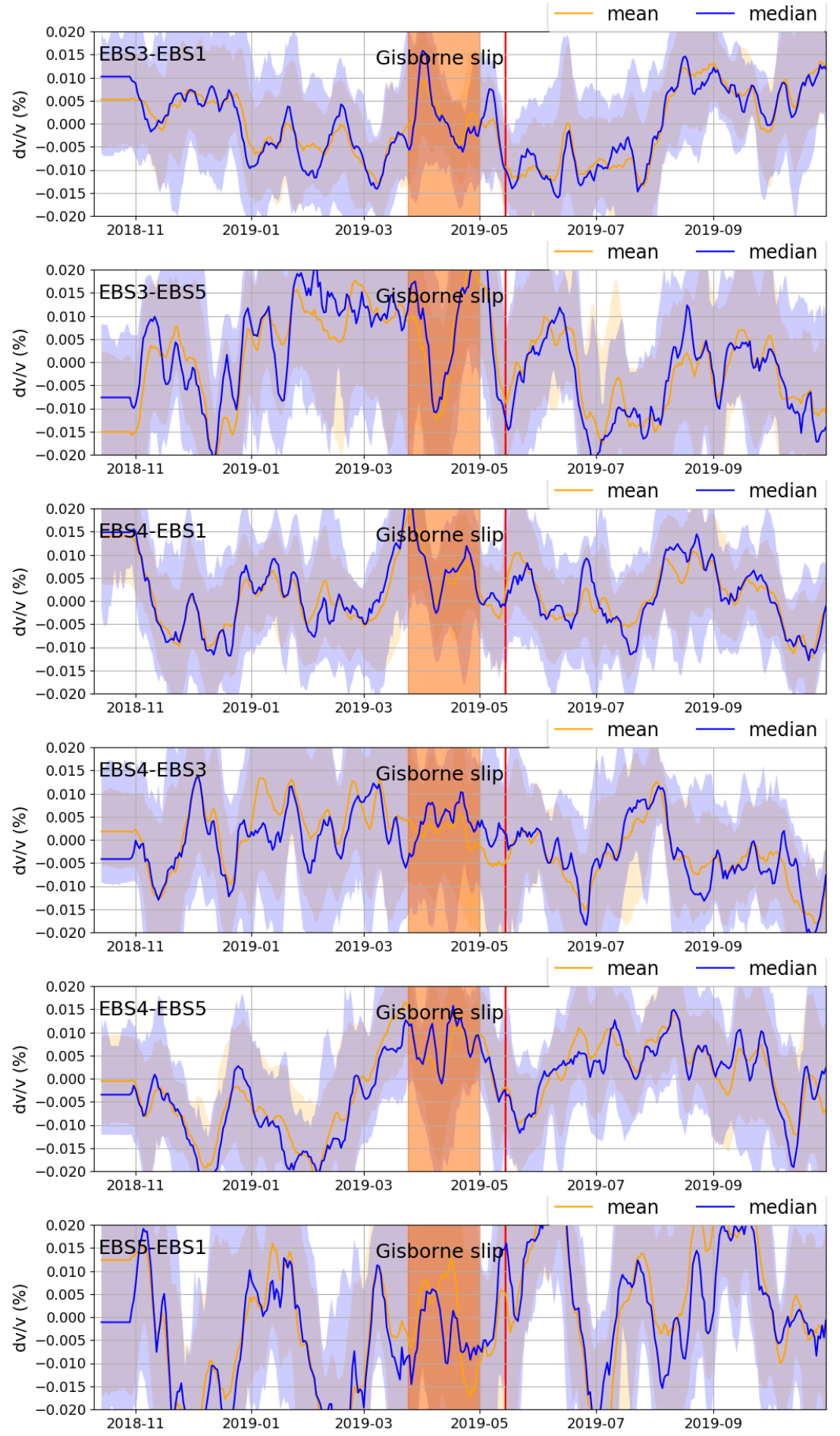

Figure B.4: Velocity variations computed from station pairs EBS3-EBS1, EBS3-EBS5, EBS4-EBS1, EBS4-EBS3, EBS4-EBS5, EBS5-EBS1, filter at 1-2.5 s. 


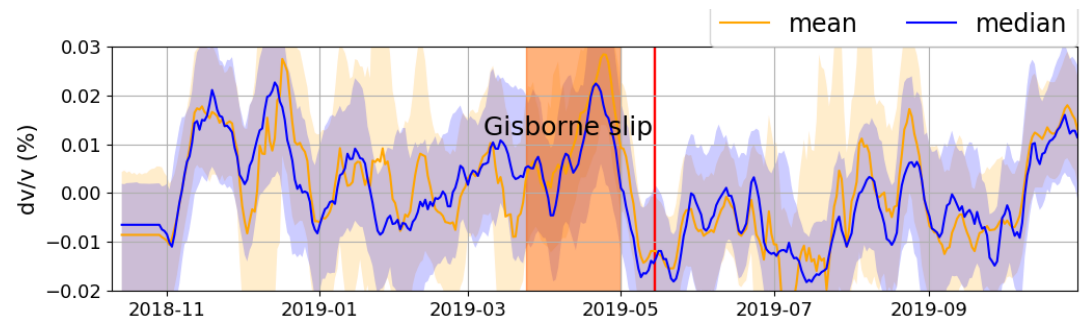

Figure B.5: Velocity variations computed using cross station correlations, single station crosscomponent correlations, and auto-correlations, filter at 2.5-14 s. 

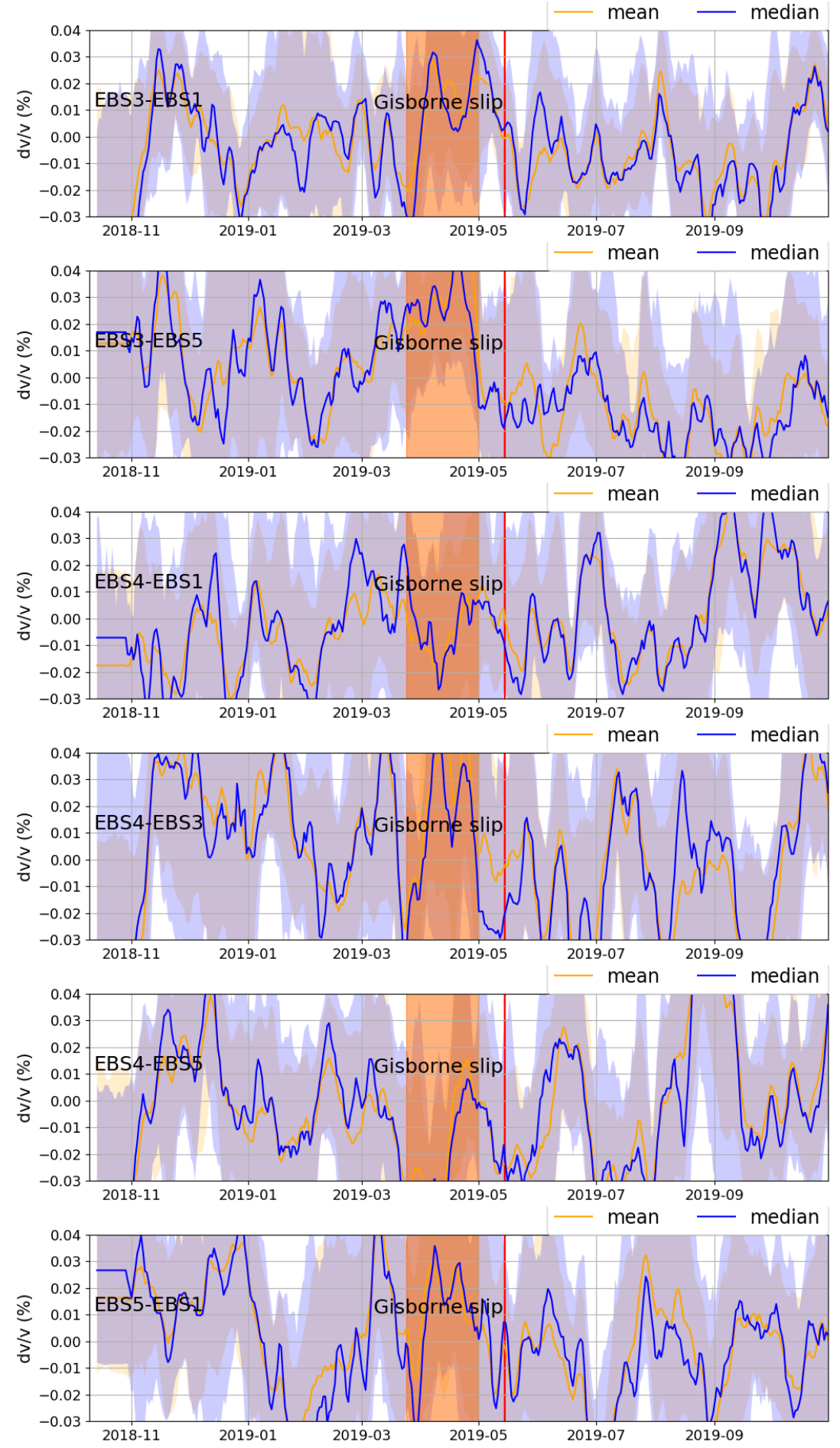

Figure B.6: Velocity variations computed from station pair EBS3-EBS1, EBS3-EBS5, EBS4-EBS1, EBS4-EBS3, EBS4-EBS5, EBS5-EBS1, filtered at 2.5-20 s. 


\section{Appendix C}

\section{Appendix C: Chapter 5}

This chapter provides appendix material for Chapter 5: Studies of shear wave velocity structure in the southwestern Okinawa Trough from continuous OBS data. 
Table C.1: Locations of the ocean bottom seismometers used in Chapter 5.

\begin{tabular}{cccc}
\hline Station & Longitude & Latitude & Altitude $(\mathrm{m})$ \\
\hline YB01 & 122.4426 & 24.9216 & -1492 \\
YB02 & 122.5019 & 24.9220 & -1514 \\
YB03 & 122.4407 & 24.9828 & -1452 \\
YB04 & 122.4414 & 25.0396 & -1255 \\
YB05 & 122.5026 & 25.0400 & -1373 \\
L1101 & 122.380 & 24.8990 & -1300 \\
L1102 & 122.499 & 24.8988 & -1503 \\
L1104 & 122.499 & 25.0180 & -1427 \\
L1105 & 122.501 & 25.0799 & -1213 \\
L1106 & 122.439 & 25.1199 & -1014 \\
Y1102 & 122.502 & 24.8415 & -1370 \\
Y1103 & 122.379 & 24.9563 & -1265 \\
Y1105 & 122.376 & 25.0781 & -1111 \\
Y1507 & 122.3512 & 25.0487 & -1129 \\
Y1508 & 122.3507 & 25.0194 & -1220 \\
Y1509 & 122.5017 & 24.8916 & -1521 \\
Y1601 & 122.3210 & 25.0188 & -1119 \\
Y1602 & 122.3454 & 25.0124 & -1185 \\
Y1603 & 122.3008 & 24.9955 & -1136 \\
Y1604 & 122.2994 & 24.9717 & -1159 \\
Y1605 & 122.3310 & 24.9696 & -1225 \\
Y1606 & 122.2988 & 24.9445 & -1243 \\
Y1701 & 122.4495 & 25.0349 & -1267 \\
Y1702 & 122.4801 & 25.0203 & -1390 \\
Y1703 & 122.4523 & 25.0000 & -1415 \\
Y1704 & 122.4804 & 24.9892 & -1435 \\
Y1705 & 122.4496 & 24.9748 & -1426 \\
Y1706 & 122.4807 & 24.9623 & -1466 \\
Y1801 & 122.4846 & 24.9733 & -1462 \\
\hline & 122.5036 & 24.9745 & -1509 \\
Y1802 & 122.5188 & 24.9570 & -1550 \\
\hline & &
\end{tabular}




\begin{tabular}{lccc}
\hline Station & Longitude & Latitude & Altitude $(\mathrm{m})$ \\
\hline Y1804 & 122.4569 & 24.8880 & -1485 \\
Y1805 & 122.5070 & 24.8802 & -1579 \\
Y1806 & 122.4799 & 24.8782 & -1496 \\
\hline
\end{tabular}

Joint inversion of the fundamental mode and the higher mode is tested, assuming the earlier arrivals are the 1st, 2nd, or 3rd overtone Scholte/Rayleigh waves. The inversion only using the earlier arrivals assuming they are the 1st overtone is also tested.
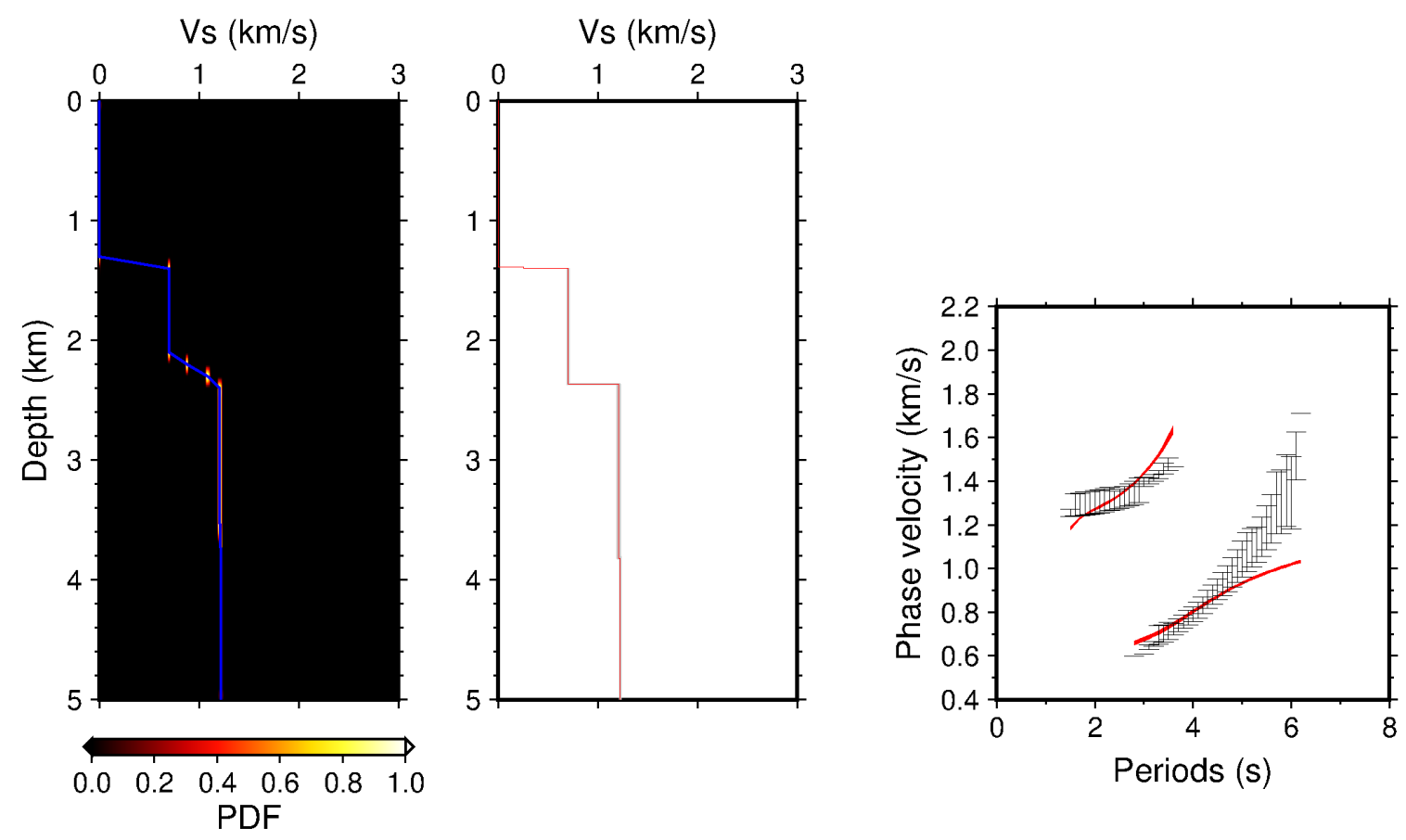

Figure C.1: Vs inversion in the center region using two modes (the two black dispersion curves in Figure 5.21b), assuming the earlier arrivals are the 2nd overtone, and corresponding data fit. The forward-modeled phase velocities (red) cannot fit with the observations (black error bars). 

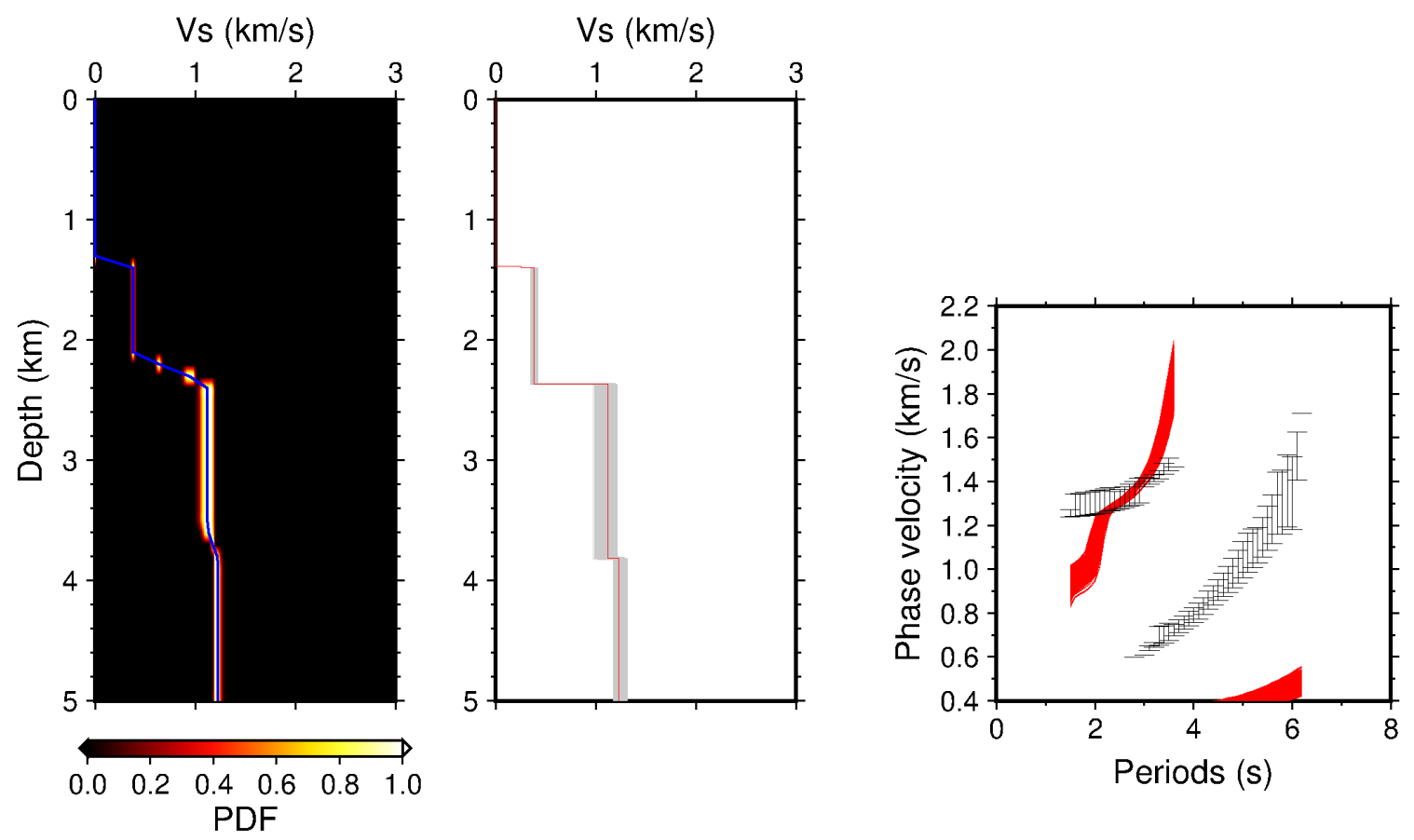

Figure C.2: Vs inversion in the center region using two modes (the two black dispersion curves in Figure 5.21b), assuming the earlier arrivals are the 3rd overtone, and corresponding data fit. The forward-modeled phase velocities (red) cannot fit with the observations (black error bars).
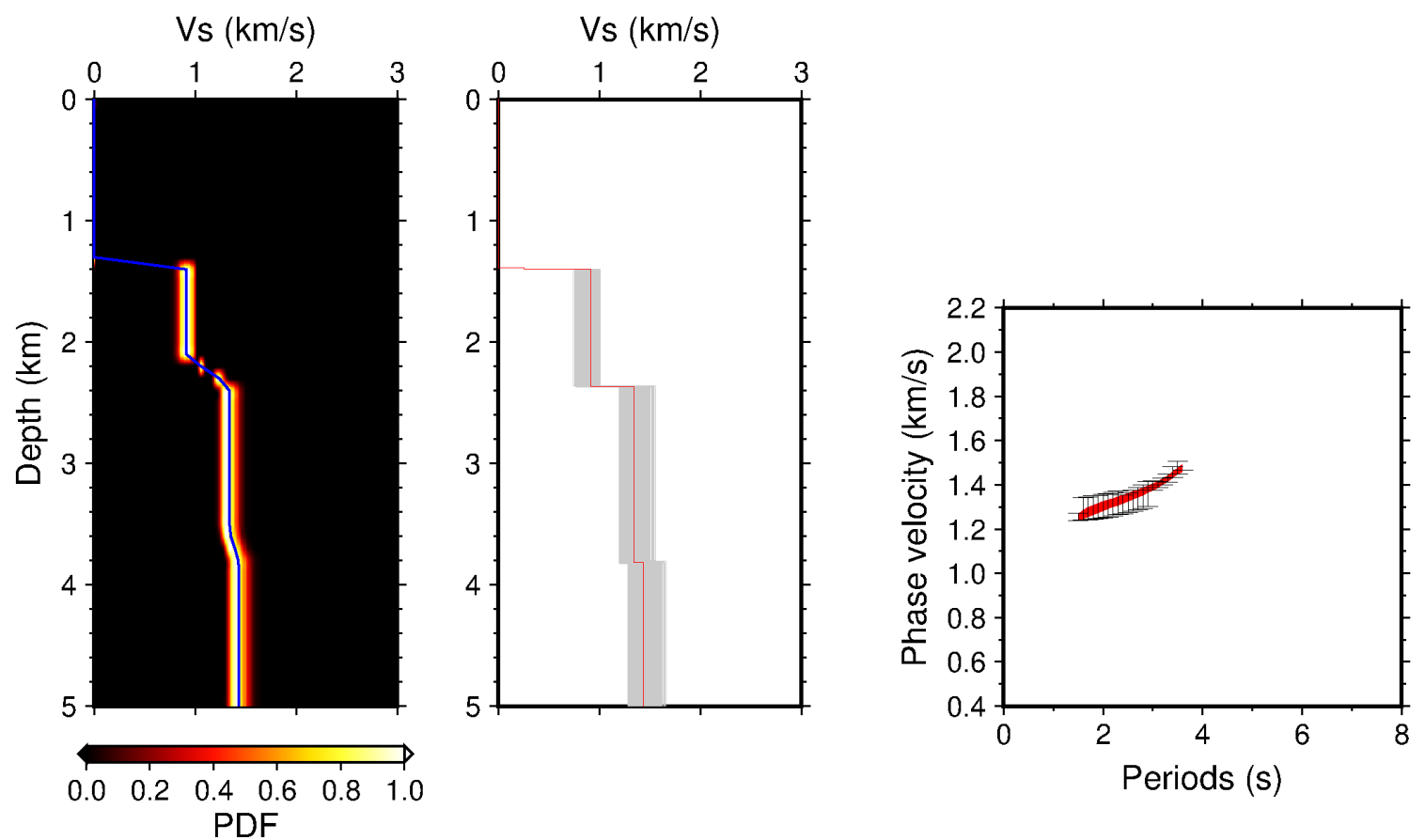

Figure C.3: Vs inversion in the center region using only the earlier arrivals (the black dispersion curve S1 in Figure 5.21b), assuming the earlier arrivals are the 1st overtone, and corresponding data fit. 


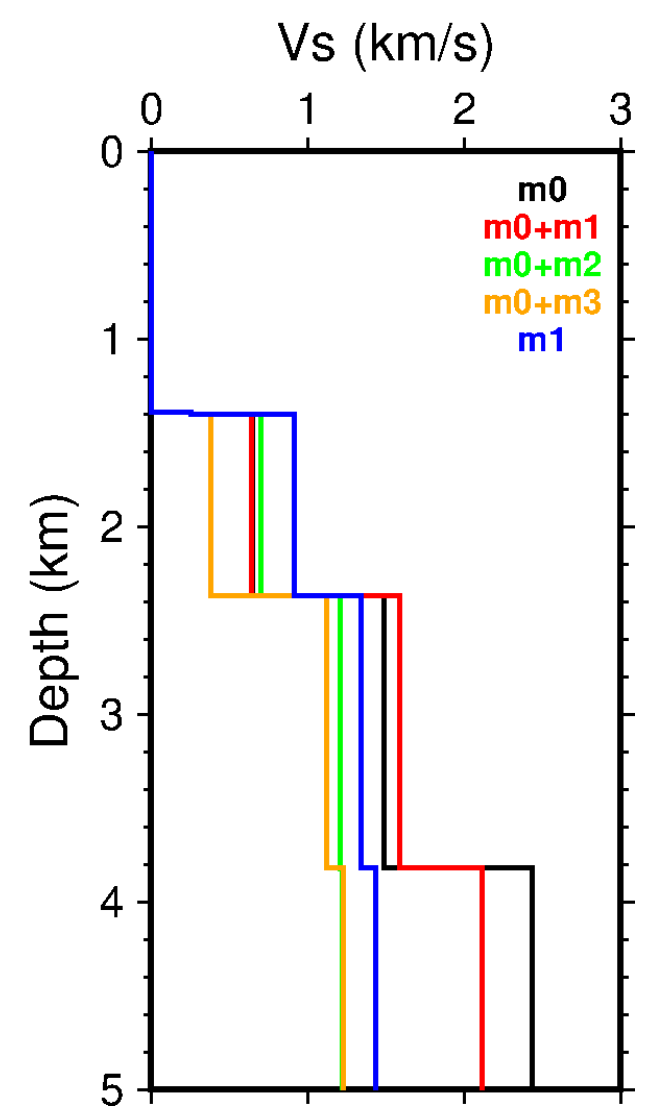

Figure C.4: Comparison of Vs models inverted with different assumptions in the center region. Here $\mathrm{m} 0$ denotes only using the fundamental mode phase velocity dispersion curve (Figure 5.22), $\mathrm{m} 0+\mathrm{m} 1$ represents using the fundamental mode and assuming the earlier arrivals are the 1st overtone (Figure 5.25), $\mathrm{m} 0+\mathrm{m} 2$ represents using the fundamental mode and assuming the earlier arrivals are the 2nd overtone (Figure C.1), $\mathrm{m} 0+\mathrm{m} 3$ represents using the fundamental mode and assuming the earlier arrivals are the 3rd overtone (Figure C.2), $\mathrm{m} 1$ represents using only the earlier arrivals and assuming the earlier arrivals are the 1st overtone (Figure C.3). 

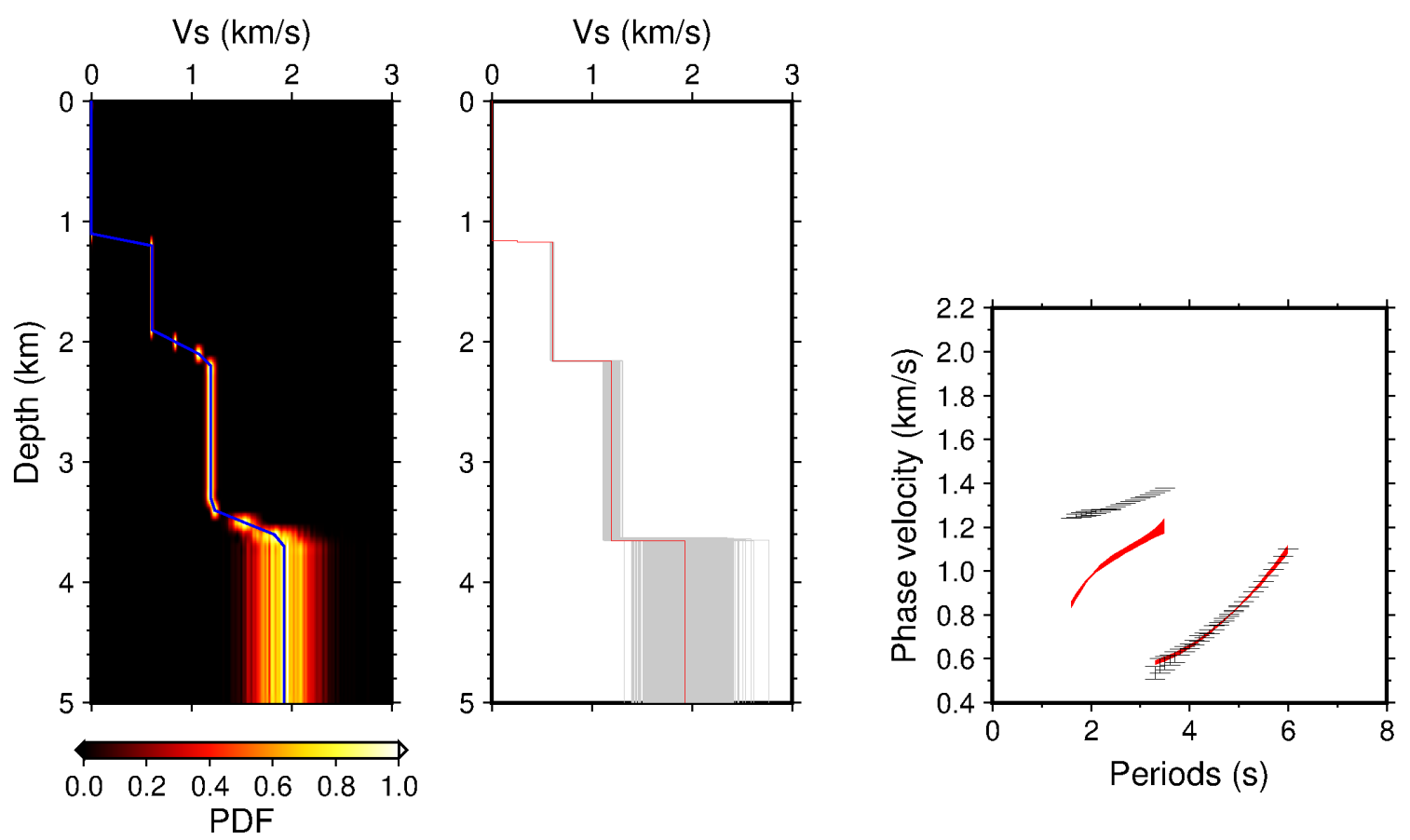

Figure C.5: Vs inversion in the northwest edge region using two modes (the two blue dispersion curves in Figure 5.21b), assuming the earlier arrivals are the 1st overtone, and corresponding data fit. The higher mode cannot fit with the observation.
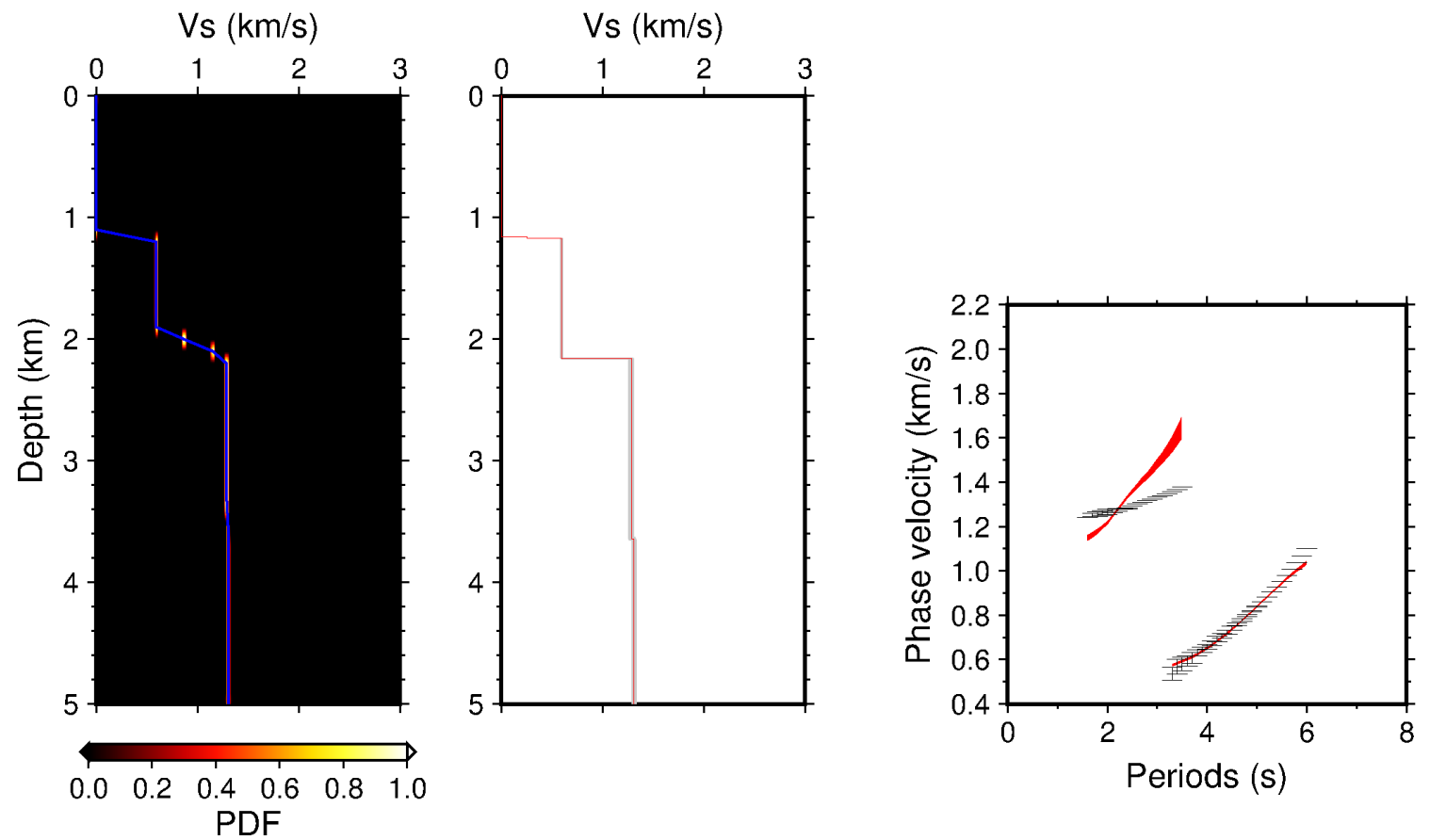

Figure C.6: Vs inversion in the northwest edge region using two modes (the two blue dispersion curves in Figure 5.21b), assuming the earlier arrivals are the 2nd overtone, and corresponding data fit. The higher mode cannot fit with the observation. 

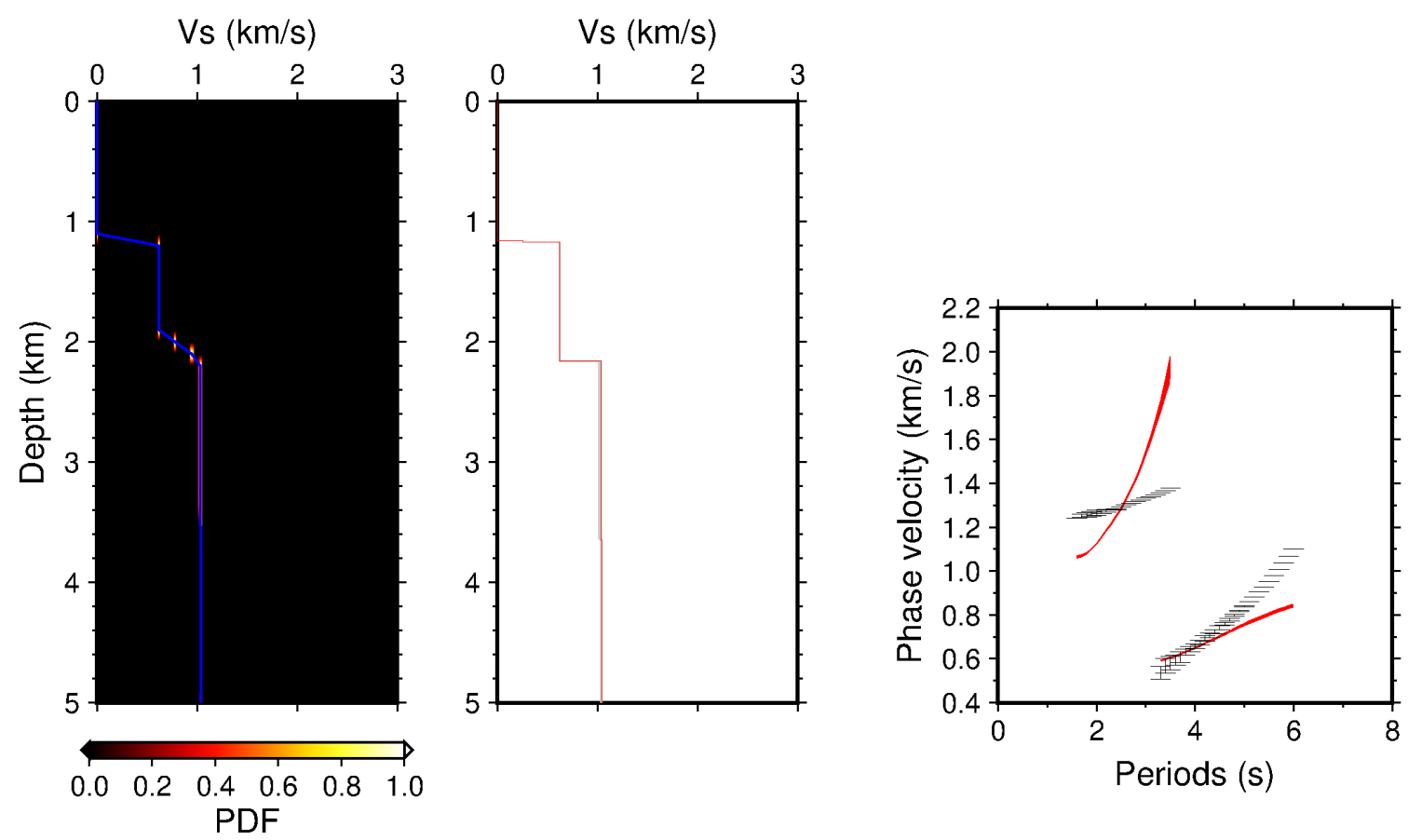

Figure C.7: Vs inversion in the northwest edge region using two modes (the two blue dispersion curves in Figure 5.21b), assuming the earlier arrivals are the 3rd overtone, and corresponding data fit. The forward-modeled phase velocities (red) cannot fit with the observations (black error bars).
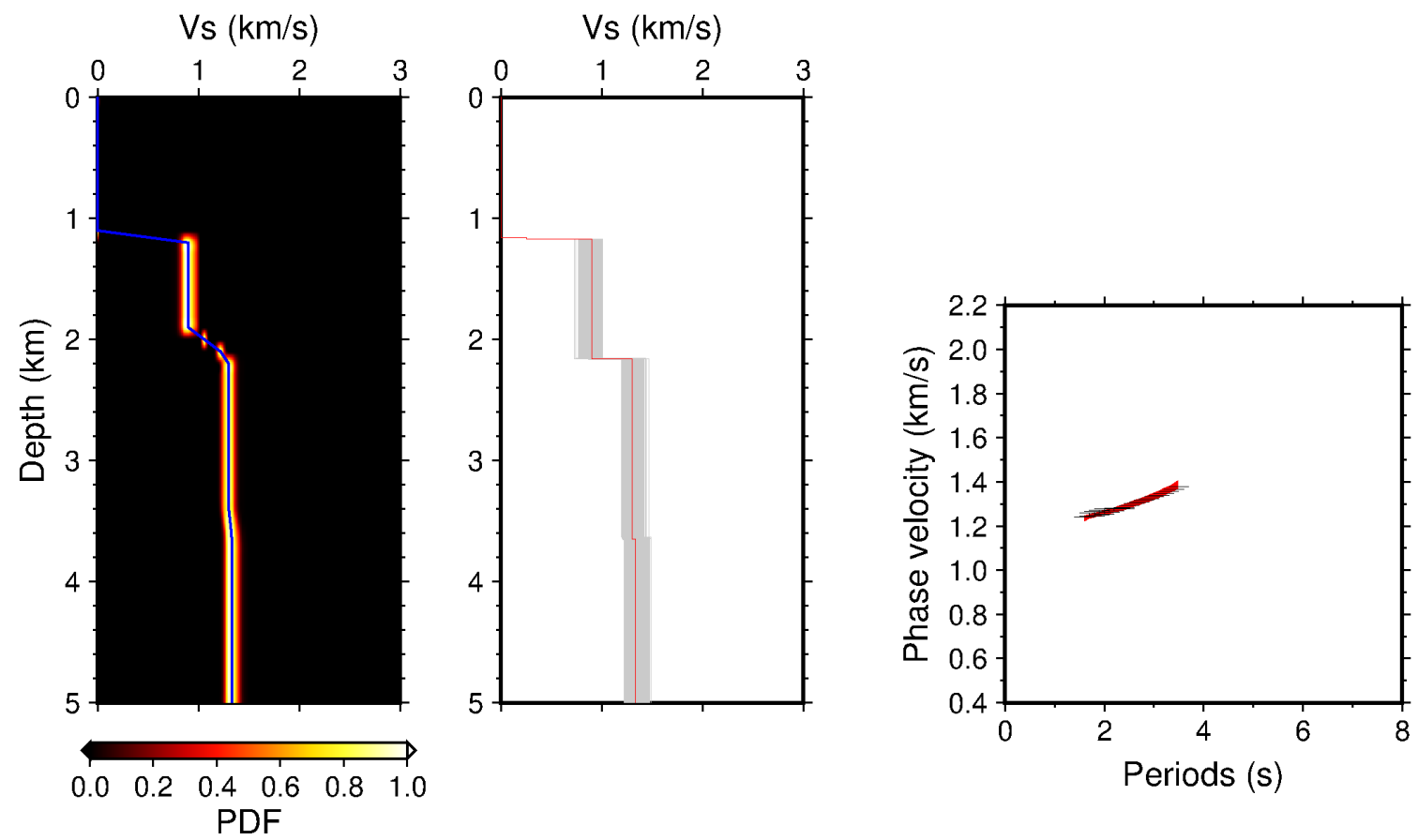

Figure C.8: Vs inversion in the northwest edge region using only the earlier arrivals (the blue dispersion curve S1 in Figure 5.21b), assuming the earlier arrivals are the 1st overtone, and corresponding data fit. 


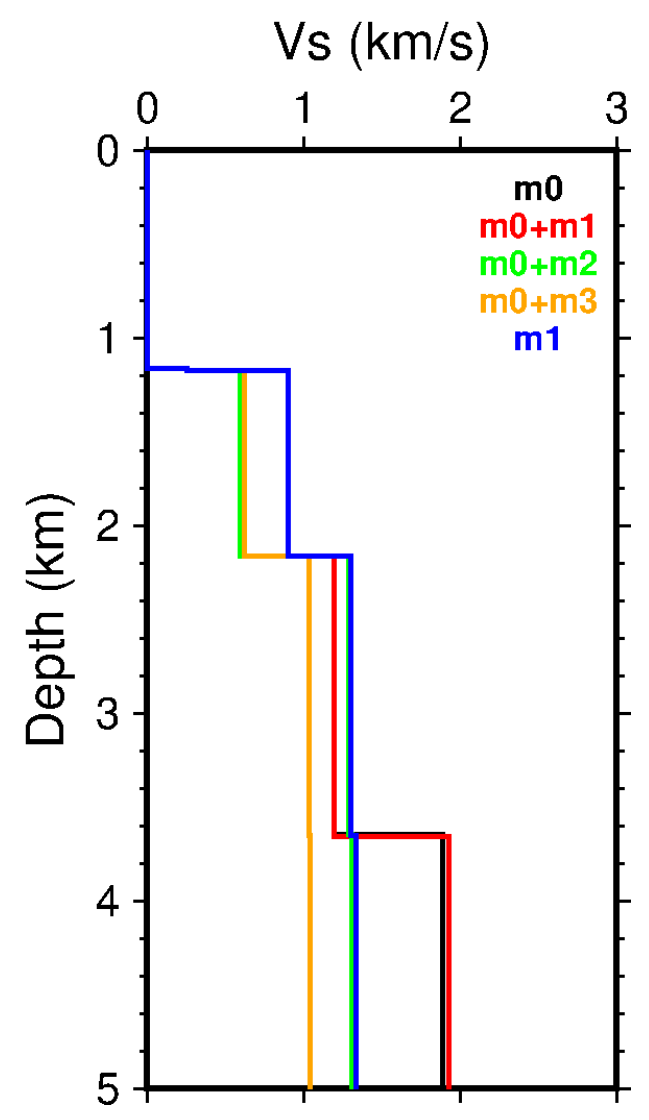

Figure C.9: Comparison of Vs models inverted with different assumptions in the northwest edge region. Here $\mathrm{m} 0$ denotes only using the fundamental mode phase velocity dispersion curve (Figure 5.23), $\mathrm{m} 0+\mathrm{m} 1$ represents using the fundamental mode and assuming the earlier arrivals are the 1st overtone (Figure C.5), $\mathrm{m} 0+\mathrm{m} 2$ represents using the fundamental mode and assuming the earlier arrivals are the 2 nd overtone (Figure C.6), $\mathrm{m} 0+\mathrm{m} 3$ represents using the fundamental mode and assuming the earlier arrivals are the 3rd overtone (Figure C.7), $\mathrm{m} 1$ represents using only the earlier arrivals and assuming the earlier arrivals are the 1st overtone (Figure C.8). 

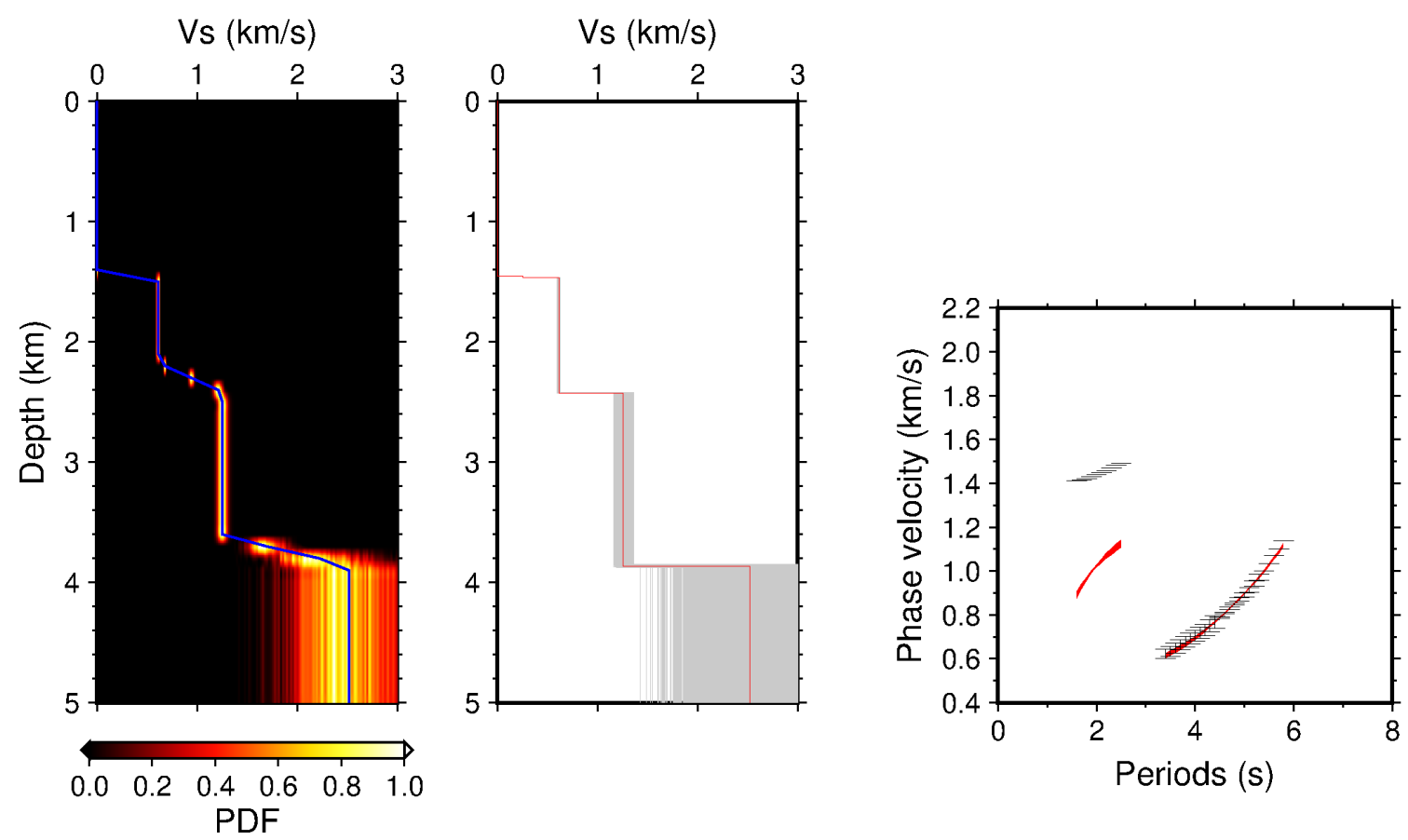

Figure C.10: Vs inversion in the south region using two modes (the two red dispersion curves in Figure 5.21b), assuming the earlier arrivals are the 1st overtone, and corresponding data fit. The higher mode cannot fit with the observation.
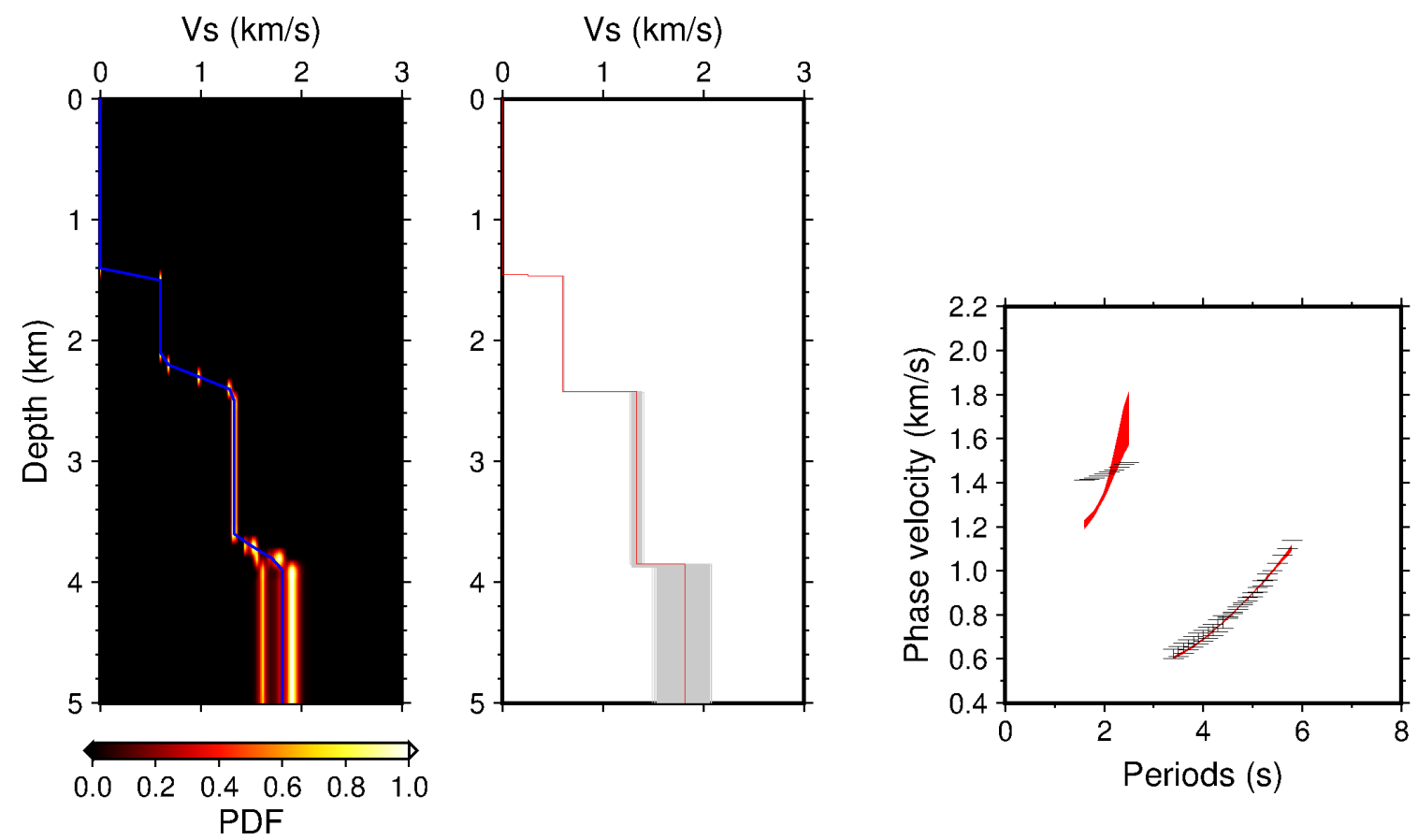

Figure C.11: Vs inversion in the south region using two modes (the two red dispersion curves in Figure 5.21b), assuming the earlier arrivals are the 2nd overtone, and corresponding data fit. The higher mode cannot fit with the observation. 

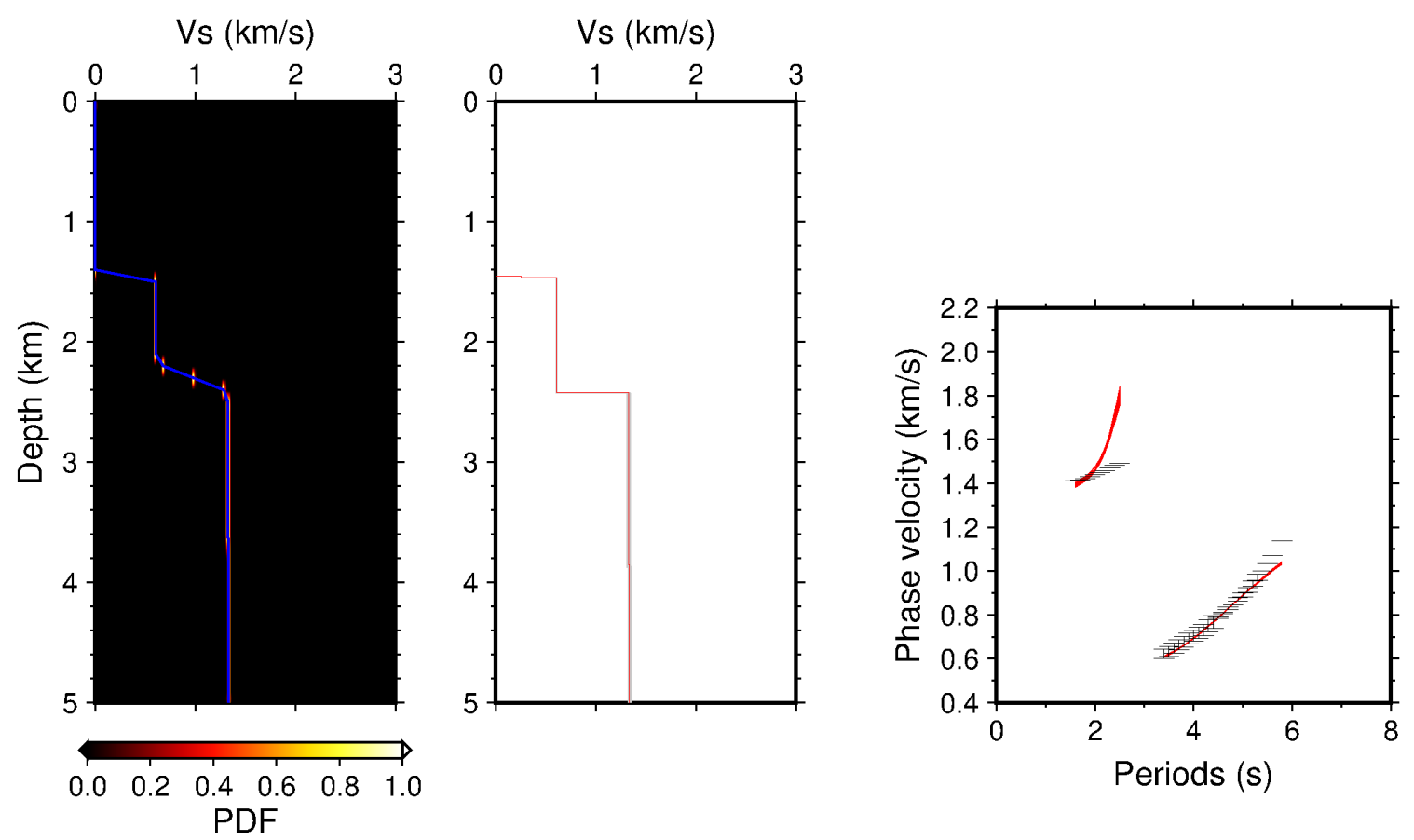

Figure C.12: Vs inversion in the south region using two modes (the two red dispersion curves in Figure 5.21b), assuming the earlier arrivals are the 3rd overtone, and corresponding data fit. The higher mode cannot fit with the observation.
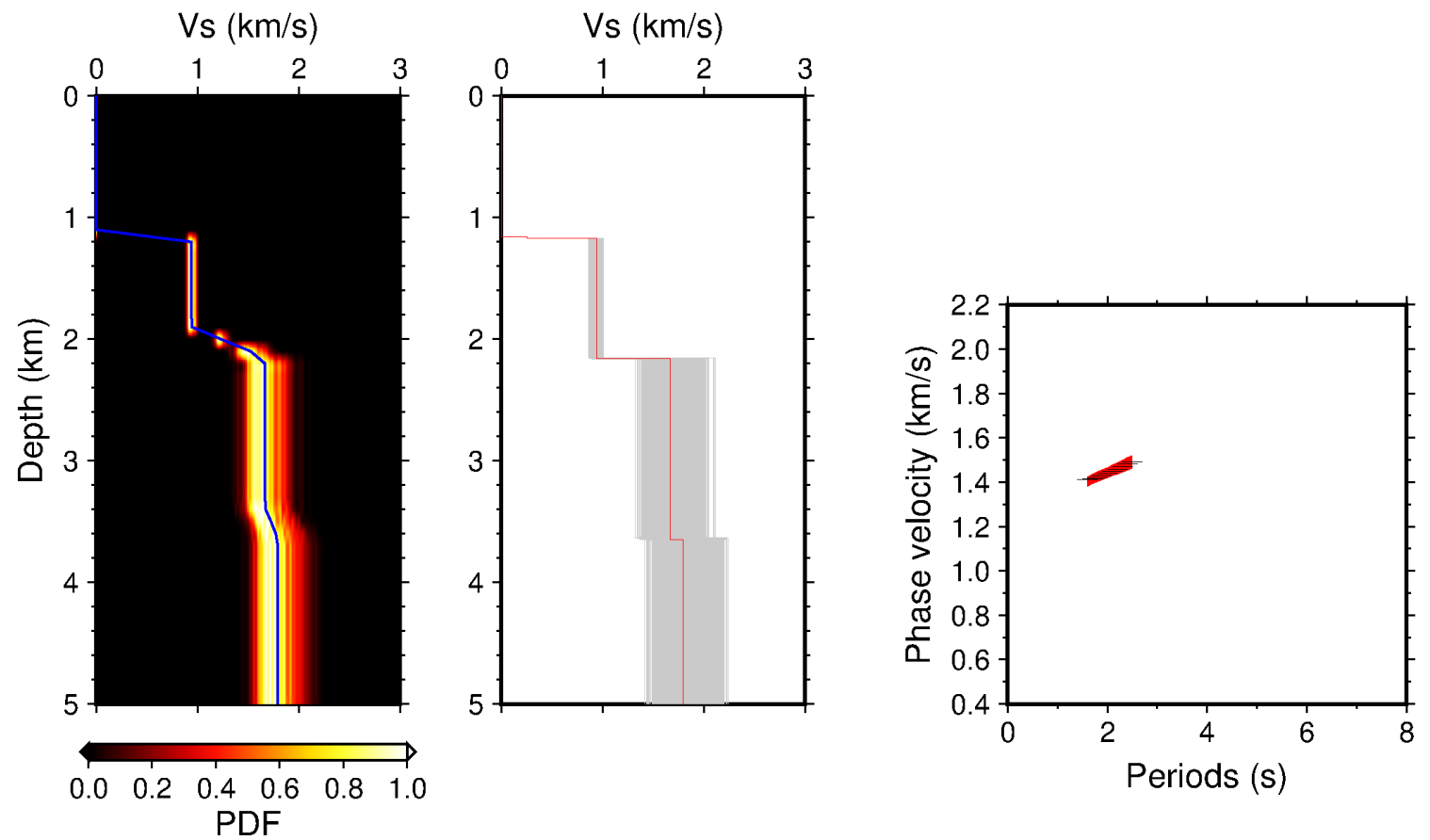

Figure C.13: Vs inversion in the south region using only the earlier arrivals (the red dispersion curve S1 in Figure 5.21b), assuming the earlier arrivals are the 1st overtone, and corresponding data fit. 


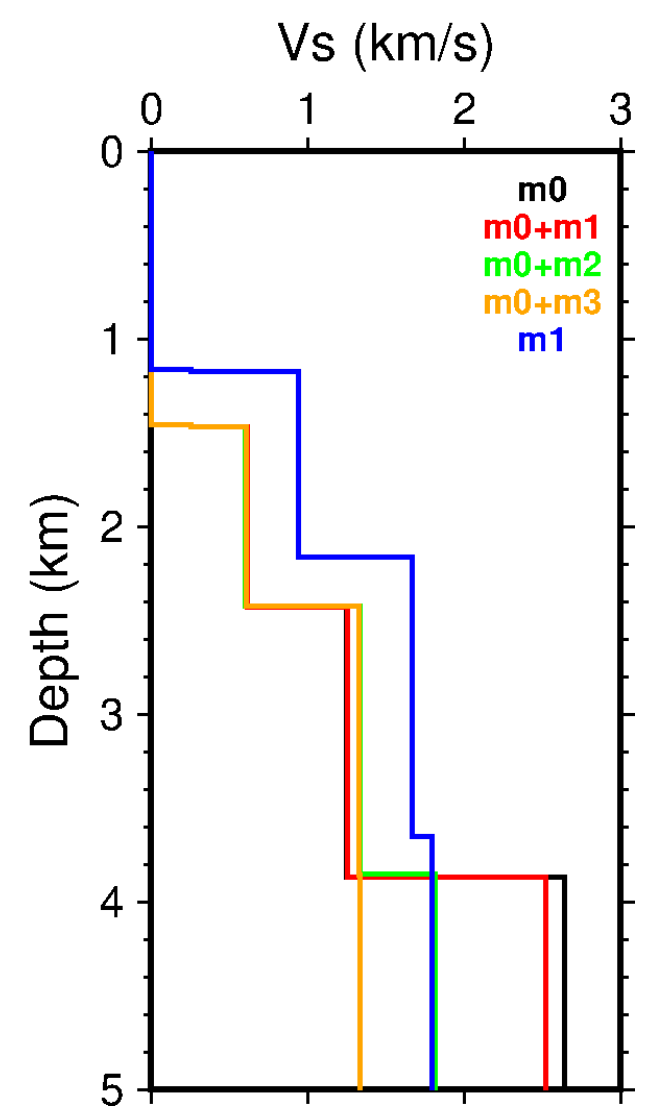

Figure C.14: Comparison of Vs models inverted with different assumptions in the south region. Here $\mathrm{m} 0$ denotes only using the fundamental mode phase velocity dispersion curve (Figure 5.24), $\mathrm{m} 0+\mathrm{m} 1$ represents using the fundamental mode and assuming the earlier arrivals are the 1st overtone (Figure C.10), $\mathrm{m} 0+\mathrm{m} 2$ represents using the fundamental mode and assuming the earlier arrivals are the 2 nd overtone (Figure C.11), $\mathrm{m} 0+\mathrm{m} 3$ represents using the fundamental mode and assuming the earlier arrivals are the 3rd overtone (Figure C.12), $\mathrm{m} 1$ represents using only the earlier arrivals and assuming the earlier arrivals are the 1st overtone (Figure C.13). 


\section{Appendix D}

\section{Appendix D: Glossary}

This chapter gives a brief description of the glossary of acronyms used in this thesis (Table D.1).

Table D.1: Acronyms used in thesis.

\begin{tabular}{|l|l|}
\hline Acronym & Definition \\
\hline OBS & Ocean Bottom Seismometer \\
LOBS & Lamont-Doherty OBS \\
EOBS & Earthquake Research Institute, University of Tokyo OBS \\
SSE & Slow Slip Event \\
HOBITSS & the Hikurangi Ocean Bottom Investigation of Tremor and Slow Slip \\
GNSS & Global Navigation Satellite System \\
CCF & cross-correlation functions \\
MSNoise & Measuring with Seismic Noise \\
SNR & Signal-Noise Ratio \\
SAC & Seismic Analysis Code \\
FTAN & frequency-time analysis \\
MCMC & Markov Chain Monte Carlo \\
CPS & Computer Programs in Seismology \\
PDF & probability density function \\
ODP & Ocean Drilling Program Site
\end{tabular}




\section{References}

Aki, K. (1981). Attenuation and Scattering of Short-Period Seismic Waves in the Lithosphere. In Husebye, E. S. and Mykkeltveit, S., editors, Identification of Seismic Sources - Earthquake or Underground Explosion, pages 515-541, Dordrecht. Springer Netherlands.

Arai, R., Kodaira, S., Yuka, K., Takahashi, T., Miura, S., and Kaneda, Y. (2017). Crustal structure of the southern Okinawa Trough: Symmetrical rifting, submarine volcano, and potential mantle accretion in the continental back-arc basin. Journal of Geophysical Research: Solid Earth, 122(1):622-641.

Audet, P., Bostock, M. G., Boyarko, D. C., Brudzinski, M. R., and Allen, R. M. (2010). Slab morphology in the Cascadia fore arc and its relation to episodic tremor and slip. Journal of Geophysical Research: Solid Earth, 115(B4).

Barker, D. H., Henrys, S., Caratori Tontini, F., Barnes, P. M., Bassett, D., Todd, E., and Wallace, L. (2018). Geophysical constraints on the relationship between seamount subduction, slow slip, and tremor at the North Hikurangi subduction zone, New Zealand. Geophysical Research Letters, 45(23):12-804.

Barker, D. H., Sutherland, R., Henrys, S., and Bannister, S. (2009). Geometry of the Hikurangi subduction thrust and upper plate, North Island, New Zealand. Geochemistry, Geophysics, Geosystems, 10(2).

Barker, D.H.N.; Wallace, L. W. K. S. M. T. S. P. (2019). Hikurangi Ocean Bottom Investigation of Tremor and Slow Slip (HOBITSS V). RV Tangaroa, Lower Hutt, N.Z.:GNS Science. GNS Science report 2019/16. 29 p. + 4 appendices; doi: $10.21420 /$ Z4FK-SK46.

Barker, P. F. and Hill, I. A. (1980). Asymmetric spreading in back-arc basins. Nature, 285(5767):652-654. 
Barnes, P. M. and de Lépinay, B. M. (1997). Rates and mechanics of rapid frontal accretion along the very obliquely convergent southern Hikurangi margin, New Zealand. Journal of Geophysical Research: Solid Earth, 102(B11):24931-24952.

Barnes, P. M., Lamarche, G., Bialas, J., Henrys, S., Pecher, I., Netzeband, G. L., Greinert, J., Mountjoy, J. J., Pedley, K., and Crutchley, G. (2010). Tectonic and geological framework for gas hydrates and cold seeps on the Hikurangi subduction margin, New Zealand. Marine Geology, 272(1-4):26-48.

Bassett, D., Sutherland, R., and Henrys, S. (2014). Slow wavespeeds and fluid overpressure in a region of shallow geodetic locking and slow slip, Hikurangi subduction margin, New Zealand. Earth and Planetary Science Letters, 389:1-13.

Bayes, T. (1763). LII. An essay towards solving a problem in the doctrine of chances. By the late Rev. Mr. Bayes, FRS communicated by Mr. Price, in a letter to John Canton, AMFR S. Philosophical transactions of the Royal Society of London, (53):370-418.

Beanland, S. and Haines, J. (1998). The kinematics of active deformation in the North Island, New Zealand, determined from geological strain rates. New Zealand Journal of Geology and Geophysics, 41(4):311-323.

Bell, R., Sutherland, R., Barker, D. H., Henrys, S., Bannister, S., Wallace, L., and Beavan, J. (2010). Seismic reflection character of the Hikurangi subduction interface, New Zealand, in the region of repeated Gisborne slow slip events. Geophysical Journal International, 180(1):34-48.

Bell, S. W., Forsyth, D. W., and Ruan, Y. (2015). Removing noise from the vertical component records of ocean-bottom seismometers: Results from year one of the Cascadia Initiative. Bulletin of the Seismological Society of America, 105(1):300-313.

Bendat, J. S. and Piersol, A. G. (2011). Random data: analysis and measurement procedures, volume 729. John Wiley \& Sons.

Bensen, G., Ritzwoller, M., Barmin, M., Levshin, A. L., Lin, F., Moschetti, M., Shapiro, N., and Yang, Y. (2007). Processing seismic ambient noise data to obtain reliable broad-band surface wave dispersion measurements. Geophysical Journal International, 169(3):1239-1260. 
Berryman, J. G. (2007). Seismic waves in rocks with fluids and fractures. Geophysical Journal International, 171(2):954-974.

Bodin, T. and Sambridge, M. (2009). Seismic tomography with the reversible jump algorithm. Geophysical Journal International, 178(3):1411-1436.

Bowden, D., Kohler, M. D., Tsai, V. C., and Weeraratne, D. S. (2016). Offshore Southern California lithospheric velocity structure from noise cross-correlation functions. Journal of Geophysical Research: Solid Earth, 121(5):3415-3427.

Brenguier, F., Campillo, M., Hadziioannou, C., Shapiro, N. M., Nadeau, R. M., and Larose, E. (2008). Postseismic Relaxation Along the San Andreas Fault at Parkfield from Continuous Seismological Observations. Science, 321(5895):14781481.

Campillo, M. and Paul, A. (2003). Long-range correlations in the diffuse seismic coda. Science, 299(5606):547-549.

Cessaro, R. K. (1994). Sources of primary and secondary microseisms. Bulletin of the Seismological Society of America, 84(1):142-148.

Chanier, F., Ferrière, J., and Angelier, J. (1999). Extensional deformation across an active margin, relations with subsidence, uplift, and rotations: The Hikurangi subduction, New Zealand. Tectonics, 18(5):862-876.

Chaves, E. J. and Schwartz, S. Y. (2016). Monitoring transient changes within overpressured regions of subduction zones using ambient seismic noise. Science advances, 2(1):e1501289.

Chen, H.-Y., Ikuta, R., Hsu, Y.-J., Tsujii, T., Ando, M., Tu, Y., Kohmi, T., Takemoto, K., Mizuno, K., Tung, H., et al. (2021). A Decade of Global Navigation Satellite System/Acoustic Measurements of Back-Arc Spreading in the Southwestern Okinawa Trough. Frontiers in Earth Science, 9:13.

Chen, H.-Y., Ikuta, R., Lin, C.-H., Hsu, Y.-J., Kohmi, T., Wang, C.-C., Yu, S.-B., Tu, Y., Tsujii, T., and Ando, M. (2018). Back-arc opening in the western end of the Okinawa Trough revealed from GNSS/acoustic measurements. Geophysical Research Letters, 45(1):137-145. 
Chen, L., Chiang, H.-T., Wu, J.-N., Chiao, L.-Y., Shyu, C.-T., Liu, C.-S., Wang, Y., and Chen, S.-C. (2020). The focus thermal study around the spreading center of southwestern Okinawa trough. Tectonophysics, 796:228649.

Chou, H.-C., Kuo, B.-Y., Hung, S.-H., Chiao, L.-Y., Zhao, D., and Wu, Y.-M. (2006). The Taiwan-Ryukyu subduction-collision complex: Folding of a viscoelastic slab and the double seismic zone. Journal of Geophysical Research: Solid Earth, 111(B4).

Christensen, N. I. and Shaw, G. H. (1970). Elasticity of mafic rocks from the MidAtlantic Ridge. Geophysical Journal International, 20(3):271-284.

Chung, S.-L., Wang, S.-L., Shinjo, R., Lee, C.-S., and Chen, C.-H. (2000). Initiation of arc magmatism in an embryonic continental rifting zone of the southernmost part of Okinawa Trough. Terra Nova, 12(5):225-230.

Clarke, D., Zaccarelli, L., Shapiro, N. M., and Brenguier, F. (2011). Assessment of resolution and accuracy of the Moving Window Cross Spectral technique for monitoring crustal temporal variations using ambient seismic noise. Geophysical Journal International, 186(2):867-882.

Coffin, M., Gahagan, L., and Lawver, L. (1998). Present-day plate boundary digital data compilation. University of Texas Institute for geophysics technical report, 174(5).

Coffin, M. F. and Eldholm, O. (1994). Large igneous provinces: crustal structure, dimensions, and external consequences. Reviews of Geophysics, 32(1):1-36.

Crawford, W. C. and Webb, S. C. (2000). Identifying and removing tilt noise from low-frequency $(<0.1 \mathrm{~Hz})$ seafloor vertical seismic data. Bulletin of the Seismological Society of America, 90(4):952-963.

De Plaen, R. S., Cannata, A., Cannavo, F., Caudron, C., Lecocq, T., and Francis, O. (2019). Temporal changes of seismic velocity caused by volcanic activity at Mt. Etna revealed by the autocorrelation of ambient seismic noise. Frontiers in Earth Science, 6:251.

De Plaen, R. S., Lecocq, T., Caudron, C., Ferrazzini, V., and Francis, O. (2016). Single-station monitoring of volcanoes using seismic ambient noise. Geophysical Research Letters, 43(16):8511-8518. 
DeMets, C., Gordon, R. G., Argus, D. F., and Stein, S. (1990). Current plate motions. Geophysical Journal International, 101(2):425-478.

DeMets, C., Gordon, R. G., Argus, D. F., and Stein, S. (1994). Effect of recent revisions to the geomagnetic reversal time scale on estimates of current plate motions. Geophysical Research Letters, 21(20):2191-2194.

Deschamps, A. and Fujiwara, T. (2003). Asymmetric accretion along the slowspreading Mariana Ridge. Geochemistry, Geophysics, Geosystems, 4(10).

Doo, W.-B., Hsu, S.-K., Wang, H.-F., Huang, Y.-S., Tsai, C.-H., Lo, C.-L., Lin, S.-S., Liang, C.-W., and Lin, Y.-J. (2019). A deep-towed magnetic survey in the southern Okinawa Trough: Implications for hydrothermal system detection. Terr. Atmos. Ocean. Sci, 30:675-683.

Douglas, A., Beavan, J., Wallace, L., and Townend, J. (2005). Slow slip on the northern Hikurangi subduction interface, New Zealand. Geophysical Research Letters, 32(16).

Dragert, H., Wang, K., and James, T. S. (2001). A silent slip event on the deeper Cascadia subduction interface. Science, 292(5521):1525-1528.

Duennebier, F. K. and Sutton, G. H. (1995). Fidelity of ocean bottom seismic observations. Marine Geophysical Researches, 17(6):535-555.

Duputel, Z., Ferrazzini, V., Brenguier, F., Shapiro, N., Campillo, M., and Nercessian, A. (2009). Real time monitoring of relative velocity changes using ambient seismic noise at the Piton de la Fournaise volcano (La Réunion) from January 2006 to June 2007. Journal of Volcanology and Geothermal Research, 184(1-2):164173.

Eberhart-Phillips, D., Bannister, S., Reyners, M., and Henrys, S. (2020). New Zealand Wide model 2.2 seismic velocity and Qs and Qp models for New Zealand.

Eberhart-Phillips, D., Han, D.-H., and Zoback, M. (1989). Empirical relationships among seismic velocity, effective pressure, porosity, and clay content in sandstone. Geophysics, 54(1):82-89. 
Flores, C. H., Uri, S., McGuire, J. J., and Collins, J. A. (2017). Observations of Seismicity and Ground Motion in the Northeast US Atlantic Margin from OceanBottom Seismometer Data. Seismological Research Letters, 88(1):23-31.

Frank, W. B., Shapiro, N. M., Husker, A. L., Kostoglodov, V., Bhat, H. S., and Campillo, M. (2015). Along-fault pore-pressure evolution during a slow-slip event in Guerrero, Mexico. Earth and Planetary Science Letters, 413:135-143.

Froment, B., Campillo, M., Chen, J., and Liu, Q. (2013). Deformation at depth associated with the 12 May $2008 \mathrm{Mw} 7.9$ Wenchuan earthquake from seismic ambient noise monitoring. Geophysical Research Letters, 40(1):78-82.

Gao, X. and Wang, K. (2014). Strength of stick-slip and creeping subduction megathrusts from heat flow observations. Science, 345(6200):1038-1041.

Gardner, G., Gardner, L., and Gregory, A. (1974). Formation velocity and density-The diagnostic basics for stratigraphic traps. Geophysics, 39(6):770-780.

Gase, A. C., Van Avendonk, H. J., Bangs, N. L., Bassett, D., Henrys, S. A., Barker, D. H., Kodaira, S., Jacobs, K. M., Luckie, T. W., Okaya, D. A., et al. (2021). Crustal structure of the northern Hikurangi margin, New Zealand: Variable accretion and overthrusting plate strength influenced by rough subduction. Journal of Geophysical Research: Solid Earth, 126(5):e2020JB021176.

Gerstoft, P. and Bromirski, P. D. (2016). "Weather bomb" induced seismic signals. Science, 353(6302):869-870.

Gosselin, J. M., Audet, P., Estève, C., McLellan, M., Mosher, S. G., and Schaeffer, A. J. (2020). Seismic evidence for megathrust fault-valve behavior during episodic tremor and slip. Science advances, 6(4):eaay5174.

Grêt, A., Snieder, R., and Scales, J. (2006). Time-lapse monitoring of rock properties with coda wave interferometry. Journal of Geophysical Research: Solid Earth, 111(B3).

Guo, Z., Chen, Y. J., Ning, J., Yang, Y., Afonso, J. C., and Tang, Y. (2016). Seismic evidence of on-going sublithosphere upper mantle convection for intra-plate volcanism in Northeast China. Earth and Planetary Science Letters, 433:31-43. 
Gutscher, M.-A., Klingelhoefer, F., Theunissen, T., Spakman, W., Berthet, T., Wang, T.-K., and Lee, C.-S. (2016). Thermal modeling of the SW Ryukyu forearc (Taiwan): Implications for the seismogenic zone and the age of the subducting Philippine Sea Plate (Huatung Basin). Tectonophysics, 692:131-142.

Hadziioannou, C., Larose, E., Coutant, O., Roux, P., and Campillo, M. (2009). Stability of monitoring weak changes in multiply scattering media with ambient noise correlation: Laboratory experiments. The Journal of the Acoustical Society of America, 125(6):3688-3695.

Hamling, I. J., Hreinsdóttir, S., Bannister, S., and Palmer, N. (2016). Off-axis magmatism along a subaerial back-arc rift: Observations from the Taupo Volcanic Zone, New Zealand. Science Advances, 2(6):e1600288.

Han, B., Zhang, X., Pei, J., and Zhang, W. (2007). Characteristics of crust-mantle in East China sea and adjacent regions. Progress in Geophysics, 22(2):376-382.

Harmon, N., Forsyth, D., and Webb, S. (2007). Using ambient seismic noise to determine short-period phase velocities and shallow shear velocities in young oceanic lithosphere. Bulletin of the Seismological Society of America, 97(6):20092023.

Harmon, N., Henstock, T., Srokosz, M., Tilmann, F., Rietbrock, A., and Barton, P. (2012). Infragravity wave source regions determined from ambient noise correlation. Geophysical research letters, 39(4).

Hasselmann, K. (1963). A statistical analysis of the generation of microseisms. Reviews of Geophysics, 1(2):177-210.

Herath, P., Stern, T. A., Savage, M. K., Bassett, D., Henrys, S., and Boulton, C. (2020). Hydration of the crust and upper mantle of the Hikurangi Plateau as it subducts at the southern Hikurangi margin. Earth and Planetary Science Letters, 541:116271.

Herrmann, R. B. (2013). Computer programs in seismology: An evolving tool for instruction and research. Seismological Research Letters, 84(6):1081-1088.

Hobiger, M., Wegler, U., Shiomi, K., and Nakahara, H. (2014). Single-station crosscorrelation analysis of ambient seismic noise: application to stations in the sur- 
roundings of the 2008 Iwate-Miyagi Nairiku earthquake. Geophysical Journal International, 198(1):90-109.

Hoffman, N. W. and Tobin, H. J. (2002). An empirical relationship between velocity and porosity for underthrust sediments of the Nankai Trough Accretionary Prism. In AGU Fall Meeting Abstracts, volume 2002, pages T62C-1328.

Huang, H.-H., Wu, Y.-M., Song, X., Chang, C.-H., Lee, S.-J., Chang, T.-M., and Hsieh, H.-H. (2014). Joint Vp and Vs tomography of Taiwan: Implications for subduction-collision orogeny. Earth and Planetary Science Letters, 392:177-191.

Husen, S. and Kissling, E. (2001). Postseismic fluid flow after the large subduction earthquake of Antofagasta, Chile. Geology, 29(9):847-850.

Hyndman, R., Wang, K., and Yamano, M. (1995). Thermal constraints on the seismogenic portion of the southwestern Japan subduction thrust. Journal of Geophysical Research: Solid Earth, 100(B8):15373-15392.

Ito, H., DeVilbiss, J., and Nur, A. (1979). Compressional and shear waves in saturated rock during water-steam transition. Journal of Geophysical Research: Solid Earth, 84(B9):4731-4735.

Jia, X. and Sun, D. (2021). Imaging the crustal interfaces along the Ryukyu arctrough system using precursors to teleseismic sP and pP. Journal of Geophysical Research: Solid Earth, 126(2):e2020JB020413.

Kaproth, B. M. and Marone, C. (2013). Slow earthquakes, preseismic velocity changes, and the origin of slow frictional stick-slip. Science, 341(6151):1229-1232.

Kearey, P., Klepeis, K., and Vine, F. (2009). Global Tectonics, 3rd editions.

Keir, D., Bastow, I. D., Corti, G., Mazzarini, F., and Rooney, T. O. (2015). The origin of along-rift variations in faulting and magmatism in the Ethiopian Rift. Tectonics, 34(3):464-477.

Kimura, M. (1985). Back-arc rifting in the Okinawa Trough. Marine and Petroleum Geology, 2(3):222-240.

Kimura, M., Kaneoka, I., UYEDA, S., HILDE, T. C., and KATO, Y. (1986). Report on DELP 1984 Cruises in the Middle Okinawa Trough. V: Topography and ge- 
ology of the central grabens and their vicinity. Bulletin of the Earthquake Research Institute, University of Tokyo, 61(2):269-310.

King, M. S. (1966). Wave velocities in rocks as a function of changes in overburden pressure and pore fluid saturants. Geophysics, 31(1):50-73.

Kinoshita, M. and Yamano, M. (1997). Hydrothermal regime and constraints on reservoir depth of the Jade site in the Mid-Okinawa Trough inferred from heat flow measurements. Journal of Geophysical Research: Solid Earth, 102(B2):31833194.

Klingelhoefer, F., Lee, C.-S., Lin, J.-Y., and Sibuet, J.-C. (2009). Structure of the southernmost Okinawa Trough from reflection and wide-angle seismic data. Tectonophysics, 466(3-4):281-288.

Kodaira, S., Iidaka, T., Kato, A., Park, J.-O., Iwasaki, T., and Kaneda, Y. (2004). High pore fluid pressure may cause silent slip in the Nankai Trough. Science, 304(5675):1295-1298.

Kuo, B.-Y., Chi, W.-C., Lin, C.-R., Chang, E. T.-Y., Collins, J., and Liu, C.-S. (2009). Two-station measurement of Rayleigh-wave phase velocities for the Huatung basin, the westernmost Philippine Sea, with OBS: implications for regional tectonics. Geophysical Journal International, 179(3):1859-1869.

Kuo, B.-Y., Crawford, W. C., Webb, S. C., Lin, C.-R., Yu, T.-C., and Chen, L. (2015). Faulting and hydration of the upper crust of the SW Okinawa Trough during continental rifting: Evidence from seafloor compliance inversion. Geophysical Research Letters, 42(12):4809-4815.

Kuo, B.-Y. and Lin, C.-R. (2014). Removing the low frequency noise on cable OBS (2/2). Central Weather Bureau.

Kuo, B.-Y., Webb, S. C., Lin, C.-R., Liang, W.-T., and Hsiao, N.-C. (2014). Removing infragravity-wave-induced noise from Ocean-Bottom Seismographs (OBS) data deployed offshore of Taiwan. Bulletin of the Seismological Society of America, 104(4):1674-1684.

Kuo-Chen, H., Wu, F. T., and Roecker, S. W. (2012). Three-dimensional P velocity structures of the lithosphere beneath Taiwan from the analysis of TAIGER and related seismic data sets. Journal of Geophysical Research: Solid Earth, 117(B6). 
Kuo-Yen, W., Mii, H.-S., and Chi-Yue, H. (2005). Age Model and Oxygen Isotope Stratigraphy of Site ODP1202 in the Southern Okinaw a Trough, Northwestern Pacific. TAO: Terrestrial, Atmospheric and Oceanic Sciences, 16(1):1.

Larose, E., Roux, P., and Campillo, M. a. (2007). Reconstruction of Rayleigh-Lamb dispersion spectrum based on noise obtained from an air-jet forcing. The Journal of the Acoustical Society of America, 122(6):3437-3444.

Lecocq, T., Caudron, C., and Brenguier, F. (2014). MSNoise, a python package for monitoring seismic velocity changes using ambient seismic noise. Seismological Research Letters, 85(3):715-726.

Lee, Y.-L. (2005). The study of active submarine volcanoes and hydrothermal vents in the southernmost part of Okinawa Trough. Master thesis, National Taiwan Ocean University.

Levshin, A., Ratnikova, L., and Berger, J. (1992). Peculiarities of surface-wave propagation across central Eurasia. Bulletin of the Seismological Society of America, 82(6):2464-2493.

Levshin, A. and Ritzwoller, M. (2001). Automated detection, extraction, and measurement of regional surface waves. In Monitoring the comprehensive nuclear-testban treaty: Surface waves, pages 1531-1545. Springer.

Lewis, K. B., Collot, J.-Y., and Lallem, S. E. (1998). The dammed Hikurangi Trough: a channel-fed trench blocked by subducting seamounts and their wake avalanches (New Zealand-France GeodyNZ Project). Basin Research, 10(4):441468.

Liang, R.-c., Liu, Y.-f., Wang, J.-q., Wang, Y., Su, T., and Wu, J. (2002). Magnetic anomaly characteristics in the middle Okinawa Trough. Advances in Marine Science, 20(4):19-26.

Lin, J.-Y., Sibuet, J.-C., Lee, C.-S., Hsu, S.-K., and Klingelhoefer, F. (2007). Origin of the southern Okinawa Trough volcanism from detailed seismic tomography. Journal of Geophysical Research: Solid Earth, 112(B8).

Lin, J.-Y., Sibuet, J.-C., Lee, C.-S., Hsu, S.-K., Klingelhoefer, F., Auffret, Y., Pelleau, P., Crozon, J., and Lin, C.-H. (2009). Microseismicity and faulting in the southwestern Okinawa Trough. Tectonophysics, 466(3-4):268-280. 
Liu, B., Li, S.-Z., Suo, Y.-H., Li, G.-X., Dai, L.-M., Somerville, I., Guo, L.-L., Zhao, S.-J., and Yu, S. (2016). The geological nature and geodynamics of the Okinawa Trough, Western Pacific. Geological Journal, 51:416-428.

Liu, Z., Huang, J., and Li, J. (2010). Comparison of four techniques for estimating temporal change of seismic velocity with passive image interferometry. Earthquake Science, 23(5):511-518.

Lobkis, O. I. and Weaver, R. L. (2001). On the emergence of the Green's function in the correlations of a diffuse field. The Journal of the Acoustical Society of America, 110(6):3011-3017.

Longuet-Higgins, M. S. (1950). A theory of the origin of microseisms. Philosophical Transactions of the Royal Society of London. Series A, Mathematical and Physical Sciences, 243(857):1-35.

Luo, Y., Yang, Y., Xu, Y., Xu, H., Zhao, K., and Wang, K. (2015). On the limitations of interstation distances in ambient noise tomography. Geophysical Journal International, 201(2):652-661.

Mantovani, E., Viti, M., Babbucci, D., Tamburelli, C., and Albarello, D. (2001). Back arc extension: which driving mechanism. J. Virtual Explorer, 3:17-45.

Martínez, F., Fryer, P., Baker, N. A., and Yamazaki, T. (1995). Evolution of backarc rifting: Mariana Trough, 20-24 N. Journal of Geophysical Research: Solid Earth, 100(B3):3807-3827.

Martinez, F., Fryer, P., and Becker, N. (2000). Geophysical characteristics of the southern Mariana Trough, 1150 N-13 40 N. Journal of Geophysical Research: Solid Earth, 105(B7):16591-16607.

Martínez-Garzón, P., Vavryčuk, V., Kwiatek, G., and Bohnhoff, M. (2016). Sensitivity of stress inversion of focal mechanisms to pore pressure changes. Geophysical Research Letters, 43(16):8441-8450.

Mavko, G. and Mukerji, T. (1995). Seismic pore space compressibility and Gassmann's relation. Geophysics, 60(6):1743-1749.

Mavko, G., Mukerji, T., and Dvorkin, J. (2020). The rock physics handbook. Cambridge university press. 
Mazengarb, C. and Speden, I. (2000). Geology of the Raukumara area, Geol.

Mazzotti, S. and Adams, J. (2004). Variability of near-term probability for the next great earthquake on the Cascadia subduction zone. Bulletin of the Seismological Society of America, 94(5):1954-1959.

Miki, M., Matsuda, T., and Otofuji, Y. (1990). Opening mode of the Okinawa Trough: paleomagnetic evidence from the South Ryukyu Arc. Tectonophysics, 175(4):335-347.

Minato, S., Tsuji, T., Ohmi, S., and Matsuoka, T. (2012). Monitoring seismic velocity change caused by the 2011 Tohoku-oki earthquake using ambient noise records. Geophysical Research Letters, 39(9).

Mochizuki, K., Yamada, T., Shinohara, M., Yamanaka, Y., and Kanazawa, T. (2008). Weak interplate coupling by seamounts and repeating $\mathrm{M}^{\sim} 7$ earthquakes. Science, 321(5893):1194-1197.

Molnar, P. and Atwater, T. (1978). Interarc spreading and Cordilleran tectonics as alternates related to the age of subducted oceanic lithosphere. Earth and Planetary Science Letters, 41(3):330-340.

Mordret, A., Jolly, A., Duputel, Z., and Fournier, N. (2010). Monitoring of phreatic eruptions using interferometry on retrieved cross-correlation function from ambient seismic noise: Results from Mt. Ruapehu, New Zealand. Journal of Volcanology and Geothermal Research, 191(1-2):46-59.

Mudiyanselage, H. and Herath, M. P. S. (2021). Lithospheric Structure of the Hikurangi Subduction Margin. PhD thesis, Open Access Victoria University of Wellington I Te Herenga Waka.

Nakajima, J. and Uchida, N. (2018). Repeated drainage from megathrusts during episodic slow slip. Nature Geoscience, 11(5):351-356.

Nakamura, M. (2004). Crustal deformation in the central and southern Ryukyu Arc estimated from GPS data. Earth and Planetary Science Letters, 217(3-4):389398.

Neef, G. and Bottrill, R. (1992). The Cenozoic geology of the Gisborne area (1: 50 000 metric sheet Y18AB), North Island, New Zealand. New Zealand journal of geology and geophysics, 35(4):515-531. 
Nicol, A., Mazengarb, C., Chanier, F., Rait, G., Uruski, C., and Wallace, L. (2007). Tectonic evolution of the active Hikurangi subduction margin, New Zealand, since the Oligocene. Tectonics, 26(4).

Nolet, G. and Dorman, L. M. (1996). Waveform analysis of Scholte modes in ocean sediment layers. Geophysical Journal International, 125(2):385-396.

Obara, K. and Kato, A. (2016). Connecting slow earthquakes to huge earthquakes. Science, 353(6296):253-257.

Ohta, Y., Freymueller, J. T., Hreinsdóttir, S., and Suito, H. (2006). A large slow slip event and the depth of the seismogenic zone in the south central Alaska subduction zone. Earth and Planetary Science Letters, 247(1-2):108-116.

Oshida, A., Tamaki, K., and Kimura, M. (1992). Origin of the magnetic anomalies in the southern Okinawa Trough. Journal of geomagnetism and geoelectricity, 44(5):345-359.

Otofuji, Y.-I. and Matsuda, T. (1987). Amount of clockwise rotation of Southwest Japan-fan shape opening of the southwestern part of the Japan Sea. Earth and Planetary Science Letters, 85(1-3):289-301.

Park, C. B., Miller, R. D., and Xia, J. (1998). Imaging dispersion curves of surface waves on multi-channel record. In SEG Technical Program Expanded Abstracts 1998, pages 1377-1380. Society of Exploration Geophysicists.

Peng, Z. and Gomberg, J. (2010). An integrated perspective of the continuum between earthquakes and slow-slip phenomena. Nature geoscience, 3(9):599-607.

Peterson, J. et al. (1993). Observations and modeling of seismic background noise.

Poupinet, G., Ellsworth, W. L., and Frechet, J. (1984). Monitoring velocity variations in the crust using earthquake doublets: An application to the Calaveras Fault, California. Journal of Geophysical Research: Solid Earth, 89(B7):5719-5731.

Ramsey, L., Hovius, N., Lague, D., and Liu, C.-S. (2006). Topographic characteristics of the submarine Taiwan orogen. Journal of Geophysical Research: Earth Surface, 111(F2). 
Ratdomopurbo, A. and Poupinet, G. (1995). Monitoring a temporal change of seismic velocity in a volcano: Application to the 1992 eruption of Mt. Merapi (Indonesia). Geophysical research letters, 22(7):775-778.

Rivet, D., Campillo, M., Radiguet, M., Zigone, D., Cruz-Atienza, V., Shapiro, N. M., Kostoglodov, V., Cotte, N., Cougoulat, G., Walpersdorf, A., et al. (2014). Seismic velocity changes, strain rate and non-volcanic tremors during the 2009-2010 slow slip event in Guerrero, Mexico. Geophysical Journal International, 196(1):447460.

Rivet, D., Campillo, M., Shapiro, N. M., Cruz-Atienza, V., Radiguet, M., Cotte, N., and Kostoglodov, V. (2011). Seismic evidence of nonlinear crustal deformation during a large slow slip event in Mexico. Geophysical Research Letters, 38(8).

Russell, J. B., Gaherty, J. B., Lin, P.-Y. P., Lizarralde, D., Collins, J. A., Hirth, G., and Evans, R. L. (2019). High-resolution constraints on Pacific upper mantle petrofabric inferred from surface-wave anisotropy. Journal of Geophysical Research: Solid Earth, 124(1):631-657.

Saffer, D. M. and Tobin, H. J. (2011). Hydrogeology and mechanics of subduction zone forearcs: Fluid flow and pore pressure. Annual Review of Earth and Planetary Sciences, 39:157-186.

Salisbury, M. H., e. a. (2002). Proceedings of the Ocean Drilling Program. Init. Rep., 195(3).

Schimmel, M. and Paulssen, H. (1997). Noise reduction and detection of weak, coherent signals through phase-weighted stacks. Geophysical Journal International, 130(2):497-505.

Schwartz, S. Y. and Rokosky, J. M. (2007). Slow slip events and seismic tremor at circum-Pacific subduction zones. Reviews of Geophysics, 45(3).

Sclater, J., Jaupart, C., and Galson, D. (1980). The heat flow through oceanic and continental crust and the heat loss of the Earth. Reviews of Geophysics, 18(1):269311.

Seno, T., Stein, S., and Gripp, A. E. (1993). A model for the motion of the Philippine Sea plate consistent with NUVEL-1 and geological data. Journal of Geophysical Research: Solid Earth, 98(B10):17941-17948. 
Sens-Schönfelder, C. and Wegler, U. (2006). Passive image interferometry and seasonal variations of seismic velocities at Merapi Volcano, Indonesia. Geophysical research letters, 33(21).

Shaddox, H. R. and Schwartz, S. Y. (2019). Subducted seamount diverts shallow slow slip to the forearc of the northern Hikurangi subduction zone, New Zealand. Geology, 47(5):415-418.

Shapiro, N. M. and Campillo, M. (2004). Emergence of broadband Rayleigh waves from correlations of the ambient seismic noise. Geophysical Research Letters, 31(7).

Shapiro, N. M., Campillo, M., Stehly, L., and Ritzwoller, M. H. (2005). Highresolution surface-wave tomography from ambient seismic noise. Science, 307(5715):1615-1618.

Shinohara, M., Fukano, T., Kanazawa, T., Araki, E., Suyehiro, K., Mochizuki, M., Nakahigashi, K., Yamada, T., and Mochizuki, K. (2008). Upper mantle and crustal seismic structure beneath the Northwestern Pacific Basin using a seafloor borehole broadband seismometer and ocean bottom seismometers. Physics of the Earth and Planetary Interiors, 170(1-2):95-106.

Shyu, C.-T. and Liu, C.-S. (2001). Heat flow of the southwestern end of the Okinawa Trough. TERRESTRIAL ATMOSPHERIC AND OCEANIC SCIENCES, 12(SUPP):305-318.

Sibuet, J.-C., Deffontaines, B., Hsu, S.-K., Thareau, N., Le Formal, J.-P., and Liu, C.-S. (1998). Okinawa trough backarc basin: Early tectonic and magmatic evolution. Journal of Geophysical Research: Solid Earth, 103(B12):30245-30267.

Sibuet, J.-C. and Hsu, S.-K. (2004). How was Taiwan created? Tectonophysics, 379(1-4):159-181.

Sibuet, J.-C., Hsu, S.-K., Shyu, C.-T., and Liu, C.-S. (1995). Structural and kinematic evolutions of the Okinawa Trough backarc basin. In Backarc basins, pages 343379. Springer.

Sibuet, J.-C., Letouzey, J., Barbier, F., Charvet, J., Foucher, J.-P., Hilde, T. W., Kimura, M., Chiao, L.-Y., Marsset, B., Muller, C., et al. (1987). Back arc extension in the Okinawa Trough. Journal of Geophysical Research: Solid Earth, 92(B13):14041-14063. 
Stehly, L., Campillo, M., and Shapiro, N. (2006). A study of the seismic noise from its long-range correlation properties. Journal of Geophysical Research: Solid Earth, 111(B10).

Stein, S. and Wysession, M. (2009). An introduction to seismology, earthquakes, and earth structure. John Wiley \& Sons.

Stern, T. (1987). Asymmetric back-arc spreading, heat flux and structure associated with the Central Volcanic Region of New Zealand. Earth and planetary science letters, 85(1-3):265-276.

Stern, T. and Benson, A. (2011). Wide-angle seismic imaging beneath an andesitic arc: Central North Island, New Zealand. Journal of Geophysical Research: Solid Earth, 116(B9).

Stern, T., Quinlan, G., and Holt, W. (1992). Basin formation behind an active subduction zone: three-dimensional flexural modelling of Wanganui Basin, New Zealand. Basin research, 4(3-4):197-214.

Suito, H. and Ozawa, S. (2009). Transient crustal deformation in the Tokai district-The Tokai slow slip event and postseismic deformation caused by the 2004 off southeast Kii Peninsula earthquake. Journal of the Seismological Society of Japan (Zisin), 61:113-135.

Sun, T., Saffer, D., and Ellis, S. (2020). Mechanical and hydrological effects of seamount subduction on megathrust stress and slip. Nature Geoscience, 13(3):249-255.

Syracuse, E. M. and Abers, G. A. (2006). Global compilation of variations in slab depth beneath arc volcanoes and implications. Geochemistry, Geophysics, Geosystems, 7(5).

TAN1405 Science Party, Todd, E. c. (2014). TAN1405 Voyage Report: Hikurangi Ocean Bottom Investigation of Tremor and Slow Slip (HOBITSS). RV Tangaroa, 10-20 May 2014:Lower Hutt, N.Z.: GNS Science. GNS Science report 2014/67 86 p. +1 CD-Rom.

Tarantola, A. (2005). Inverse problem theory and model parameter estimation: Society for Industrial and Applied Mathematics. SIAM. Philadelphia. 
Taylor, B. (2013). Backarc basins: Tectonics and magmatism. Springer Science \& Business Media.

Tian, Y. and Ritzwoller, M. H. (2015). Directionality of ambient noise on the Juan de Fuca plate: Implications for source locations of the primary and secondary microseisms. Geophysical Journal International, 201(1):429-443.

Todd, E. K., Schwartz, S. Y., Mochizuki, K., Wallace, L. M., Sheehan, A. F., Webb, S. C., Williams, C. A., Nakai, J., Yarce, J., Fry, B., Henrys, S., and Ito, Y. (2018). Earthquakes and Tremor Linked to Seamount Subduction During Shallow Slow Slip at the Hikurangi Margin, New Zealand. Journal of Geophysical Research: Solid Earth, 123(8):6769-6783.

Tsai, C., Lee, C., and Wang, C. (1998). New discovery of submarine volcanoes in the westernmost part of the Okinawa Trough. In Workshop for Oceanographic Research Programs Supported by National Science Council (Taiwan).

Tsai, V. C. (2009). On establishing the accuracy of noise tomography traveltime measurements in a realistic medium. Geophysical Journal International, 178(3):1555-1564.

Tukey, J. W. (1962). The future of data analysis. The annals of mathematical statistics, 33(1):1-67.

Walcott, R. I. (1984). The kinematics of the plate boundary zone through New Zealand: a comparison of short- and long-term deformations. Geophysical Journal International, 79(2):613-633.

Wallace, L., Saffer, D., Barnes, P., Pecher, I., Petronotis, K., and LeVay, L. (2019). Site U1519.

Wallace, L. M. and Beavan, J. (2010). Diverse slow slip behavior at the Hikurangi subduction margin, New Zealand. Journal of Geophysical Research: Solid Earth, 115(B12).

Wallace, L. M., Beavan, J., McCaffrey, R., and Darby, D. (2004). Subduction zone coupling and tectonic block rotations in the North Island, New Zealand. Journal of Geophysical Research: Solid Earth, 109(B12). 
Wallace, L. M., Reyners, M., Cochran, U., Bannister, S., Barnes, P. M., Berryman, K., Downes, G., Eberhart-Phillips, D., Fagereng, A., Ellis, S., et al. (2009). Characterizing the seismogenic zone of a major plate boundary subduction thrust: Hikurangi Margin, New Zealand. Geochemistry, Geophysics, Geosystems, 10(10).

Wallace, L. M., Webb, S. C., Ito, Y., Mochizuki, K., Hino, R., Henrys, S., Schwartz, S. Y., and Sheehan, A. F. (2016). Slow slip near the trench at the Hikurangi subduction zone, New Zealand. Science, 352(6286):701-704.

Wang, G., Jiang, S., Li, S., Zhang, H., Cao, W., Zhao, F., Lei, J., and Gao, S. (2020). Crustal nature and lithospheric structure of the Okinawa Trough. Geological Journal, 55(9):6106-6122.

Wang, K. and Bilek, S. L. (2011). Do subducting seamounts generate or stop large earthquakes? Geology, 39(9):819-822.

Wang, K., Luo, Y., and Yang, Y. (2016). Correction of phase velocity bias caused by strong directional noise sources in high-frequency ambient noise tomography: a case study in Karamay, China. Geophysical Journal International, 205(2):715-727.

Wang, K.-L., Chung, S.-L., Chen, C.-H., Shinjo, R., Yang, T. F., and Chen, C.-H. (1999). Post-collisional magmatism around northern Taiwan and its relation with opening of the Okinawa Trough. Tectonophysics, 308(3):363-376.

Wang, T. K., Lin, S.-F., Liu, C.-S., and Wang, C.-S. (2004). Crustal structure of the southernmost Ryukyu subduction zone: OBS, MCS and gravity modelling. Geophysical Journal International, 157(1):147-163.

Wapenaar, K., Draganov, D., Snieder, R., Campman, X., and Verdel, A. (2010). Tutorial on seismic interferometry: Part 1 -Basic principles and applications. Geophysics, 75(5):75A195-75A209.

Warren-Smith, E., Fry, B., Wallace, L., Chon, E., Henrys, S. A., Sheehan, A. F., Mochizuki, K., Schwartz, S. Y., and Lebedev, S. (2019). Episodic stress and fluid pressure cycling in subducting oceanic crust during slow slip. Journal of Geophysical Research: Solid Earth.

Watanabe, T., Epp, D., Uyeda, S., Langseth, M., and Yasui, M. (1970). Heat flow in the Philippine Sea. Tectonophysics, 10(1-3):205-224. 
Webb, S. C. (1998). Broadband seismology and noise under the ocean. Reviews of Geophysics, 36(1):105-142.

Webb, S. C. (2007). The Earth's 'hum'is driven by ocean waves over the continental shelves. Nature, 445(7129):754-756.

Webb, S. C. and Crawford, W. C. (2010). Shallow-water broadband OBS seismology. Bulletin of the Seismological Society of America, 100(4):1770-1778.

Wegler, U. and Sens-Schönfelder, C. (2007). Fault zone monitoring with passive image interferometry. Geophysical Journal International, 168(3):1029-1033.

Williams, C. A., Eberhart-Phillips, D., Bannister, S., Barker, D. H. N., Henrys, S., Reyners, M., and Sutherland, R. (2013). Revised Interface Geometry for the Hikurangi Subduction Zone, New Zealand. Seismological Research Letters, 84(6):1066-1073.

Woods, K., Wallace, L. M., Webb, S. C., Ito, Y., Savage, M. K., Chadwell, C. D., Williams, C. A., Hino, R., Mochizuki, K., Warren-Smith, E., et al. (2020). Updip Migration of Slow Slip Revealed Through Seafloor Geodesy during 2019 East Coast Slow Slip at the Hikurangi Margin, New Zealand. In AGU Fall Meeting 2020. AGU.

Wu, F. T., Davey, F. J., and Okaya, D. A. (2007). Taiwan and South Island, New Zealand: A comparison of continental collisional orogenies. Washington DC American Geophysical Union Geophysical Monograph Series, 175:329-346.

Wu, J.-N., Chiang, H.-T., Chiao, L.-Y., Shyu, C.-T., Liu, C.-S., Wang, Y., and Chen, S.-C. (2019). Revisiting the data reduction of seafloor heat-flow measurement: The example of mapping hydrothermal venting site around Yonaguni Knoll IV in the South Okinawa Trough. Tectonophysics, 767:228159.

Yamano, M. (1988). Heat flow. The Ocean Basins and Margins, B, 7:523-557.

Yamano, M., Uyeda, S., Foucher, J.-P., and Sibuet, J.-C. (1989). Heat flow anomaly in the middle Okinawa Trough. Tectonophysics, 159(3-4):307-318.

Yang, X., Luo, Y., Xu, H., and Zhao, K. (2020). Shear wave velocity and radial anisotropy structures beneath the central Pacific from surface wave analysis of OBS records. Earth and Planetary Science Letters, 534:116086. 
Yang, Y., Ritzwoller, M. H., Lin, F.-C., Moschetti, M., and Shapiro, N. M. (2008). Structure of the crust and uppermost mantle beneath the western United States revealed by ambient noise and earthquake tomography. Journal of Geophysical Research: Solid Earth, 113(B12).

Yao, H., van Der Hilst, R. D., and De Hoop, M. V. (2006). Surface-wave array tomography in SE Tibet from ambient seismic noise and two-station analysis-I. Phase velocity maps. Geophysical Journal International, 166(2):732-744.

Yarce, J., Sheehan, A. F., Nakai, J. S., Schwartz, S. Y., Mochizuki, K., Savage, M. K., Wallace, L. M., Henrys, S. A., Webb, S. C., Ito, Y., Abercrombie, R. E., Fry, B., Shaddox, H., and Todd, E. K. (2019). Seismicity at the Northern Hikurangi Margin, New Zealand, and Investigation of the Potential Spatial and Temporal Relationships With a Shallow Slow Slip Event. Journal of Geophysical Research: Solid Earth, 124(5):4751-4766.

Yasui, M., Kishii, T., Watanabe, T., and Uyeda, S. (2012). Heat flow in the Sea of Japan. The crust and upper mantle of the Pacific area, 12:3-16.

Yates, A. (2018). Seismic velocity changes at White Island volcano, New Zealand, using ten years of ambient noise interferometry.

Yates, A. S., Savage, M. K., Jolly, A. D., Caudron, C., and Hamling, I. J. (2019). Volcanic, Coseismic, and Seasonal Changes Detected at White Island (Whakaari) Volcano, New Zealand, Using Seismic Ambient Noise. Geophysical Research Letters, 46(1):99-108.

Yohler, R., Bartlow, N., Wallace, L., and Williams, C. (2019). Time-dependent behavior of a near-trench slow-slip event at the Hikurangi subduction zone. Geochemistry, Geophysics, Geosystems, 20(8):4292-4304.

Yu, Z., Zhai, S., Guo, K., Zhou, Y., and Zong, T. (2016). Helium isotopes in volcanic rocks from the Okinawa Trough-impact of volatile recycling and crustal contamination. Geological Journal, 51:376-386.

Zal, H. J., Jacobs, K., Savage, M. K., Yarce, J., Mroczek, S., Graham, K., Todd, E. K., Nakai, J., Iwasaki, Y., Sheehan, A., Mochizuki, K., Wallace, L., Schwartz, S., Webb, S., and Henrys, S. (2020). Temporal and spatial variations in seismic 
anisotropy and VP/VS ratios in a region of slow slip. Earth and Planetary Science Letters, 532:115970.

Zha, Y. and Webb, S. C. (2016). Crustal shear velocity structure in the Southern Lau Basin constrained by seafloor compliance. Journal of Geophysical Research: Solid Earth, 121(5):3220-3237. 